\title{
ESTUDIO DE LA DINÁMICA DEL VUELO DE UN \\ DECELERADOR AERODINÁMICO BASADO EN EL \\ CONCEPTO DE PARARROTOR
}

\author{
Tesis de Doctorado
}

JOAQUÍN PIECHOCKI

\author{
Presentada ante la Facultad de Ingeniería de la \\ Universidad Nacional de La Plata
}

como requisito para la obtención del grado académico de

\section{DOCTOR EN INGENIERÍA}

Dirección de Tesis:

Ángel Sanz Andrés, Vicente Nadal Mora.

Jurado de Tesis:

Jorge Luis Lassig, Sergio Amado Elaskar, Sergio Preidikman

Fecha de defensa oral y pública:

25 de abril de 2012 


\section{ÍNDICE}

NOMENCLATURA

10

RESUMEN

CAPÍTULO 1

INTRODUCCIÓN 17

1.1 INTRODUCCIÓN AL CONCEPTO DE PARARROTOR 17

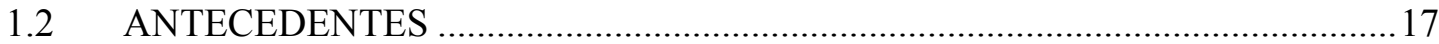

1.2.1 Antecedentes en investigaciones sobre casos biológicos................................ 18

1.2.2 Antecedentes en investigaciones sobre casos de aplicación ..............................20

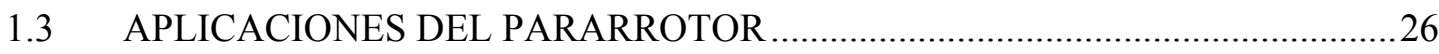

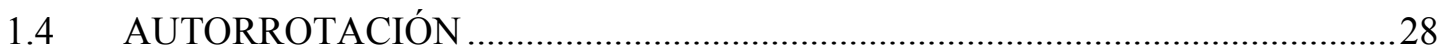

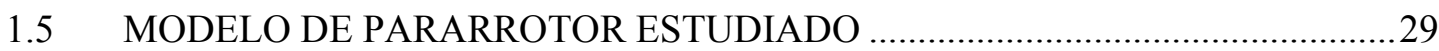

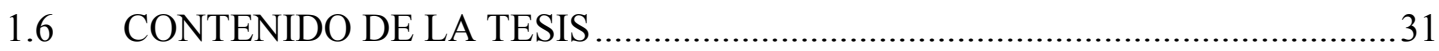

CAPÍTULO 2

ESTUDIO TEÓRICO DEL EFECTO DEL DESPLAZAMIENTO DEL PLANO QUE CONTIENE A LAS PALAS CON RESPECTO AL CENTRO DE MASAS EN LA DINÁMICA DEL VUELO DE UN DESACELERADOR AERODINÁMICO DE ALAS

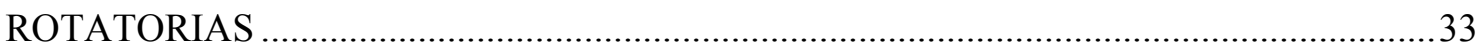

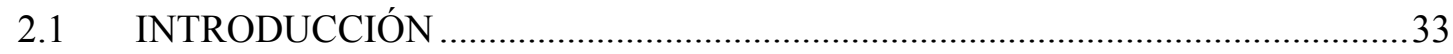

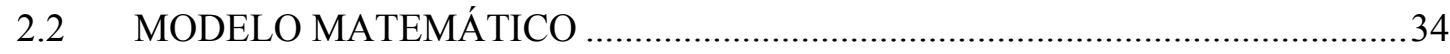




\subsection{ECUACIONES DE LA DINÁMICA DEL PARARROTOR …...............................36}

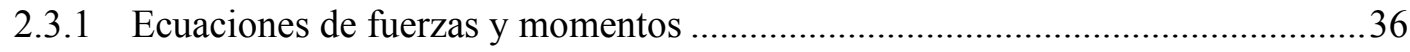

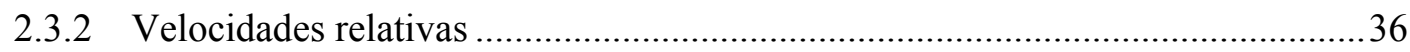

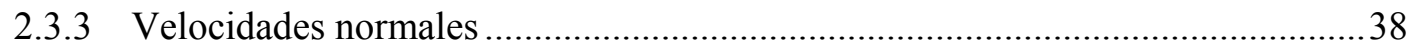

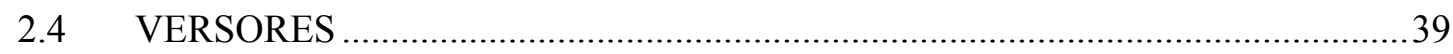

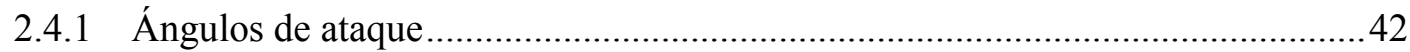

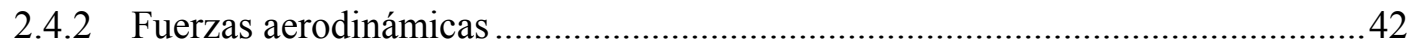

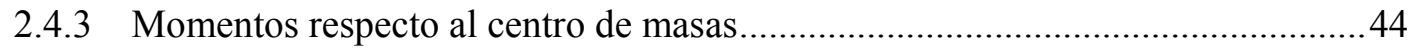

2.4.4 Linealización del sistema de ecuaciones.......................................................45

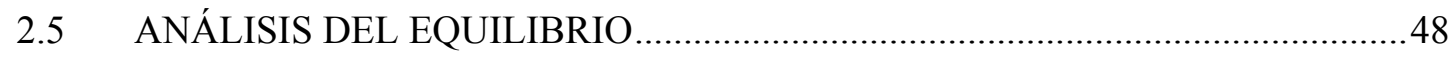

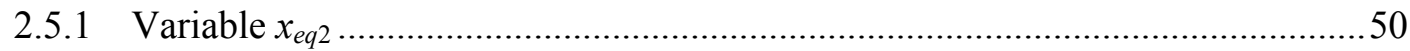

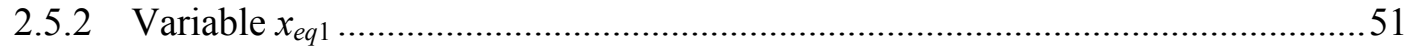

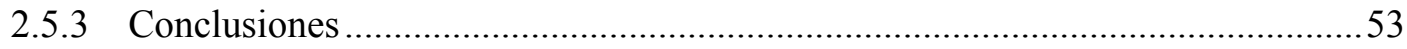

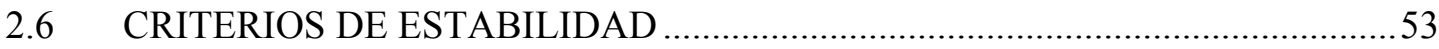

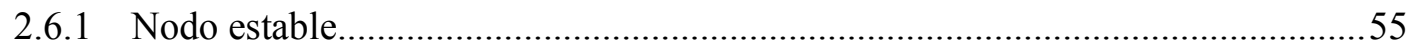

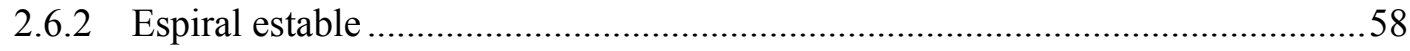

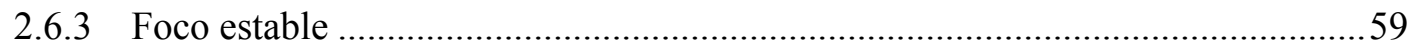

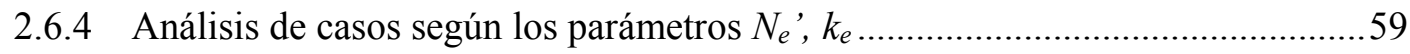

2.6.5 Caso A - Estabilidad inercial. Eje principal mayor próximo al eje de giro $-I_{3}>$ $I_{2}, I_{1} \quad 61$ 
2.6.6 Caso B - Estabilización aerodinámica. Eje principal intermedio próximo al eje de giro $-I_{2}>I_{3}>I_{1}$

2.6.7 Caso C - Estabilización aerodinámica. Eje principal intermedio próximo al eje de giro $-I_{1}>I_{3}>I_{2}$.

2.6.8 Caso D - Estabilidad inercial. Eje principal menor próximo al eje de giro $-I_{1}, I_{2}>$ $I_{3} \quad 66$

2.7 ANGULO DE NUTACIÓN .67

2.8 RESUMEN .69

2.9 EJEMPLOS NUMÉRICOS .69

2.10 CONCLUSIÓN .77

\section{CAPÍTULO 3}

ESTUDIO DEL EFECTO DEL DESPLAZAMIENTO DEL PLANO QUE CONTIENE A LAS PALAS CON RESPECTO AL CENTRO DE MASAS A PARTIR DEL DESARROLLO DE UNA RUTINA DE SIMULACIÓN DE LA DINÁMICA DEL PARARROTOR

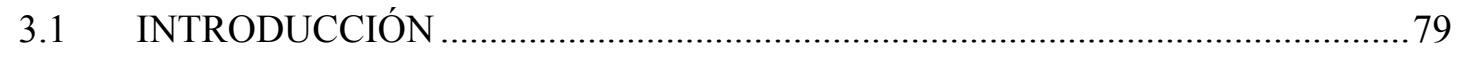

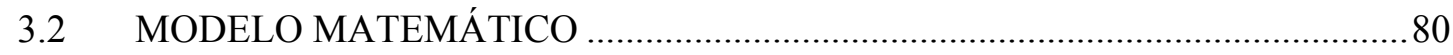

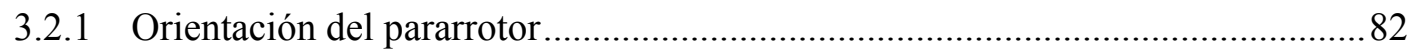

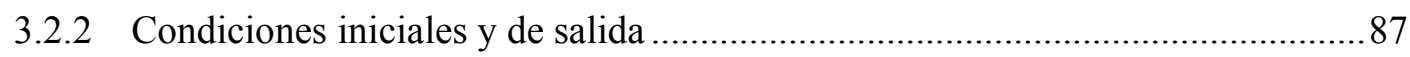

3.2.3 Ecuaciones de la dinámica del pararrotor ................................................... 92

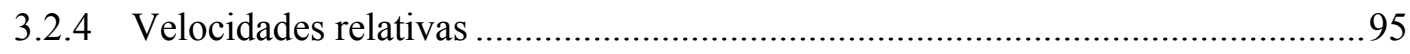

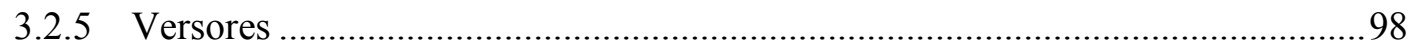

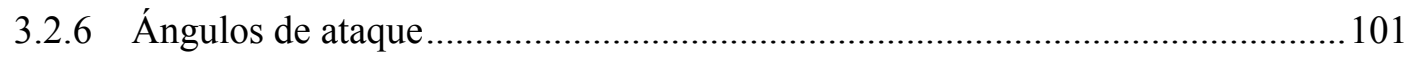




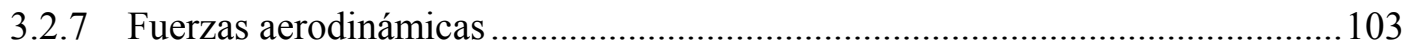

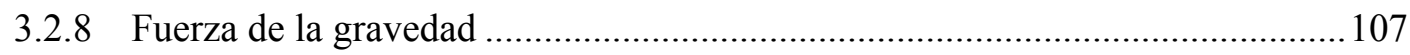

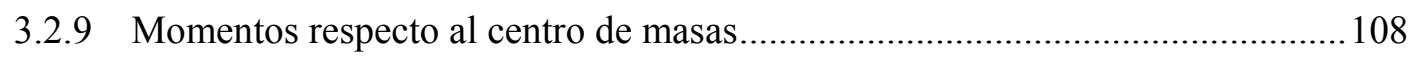

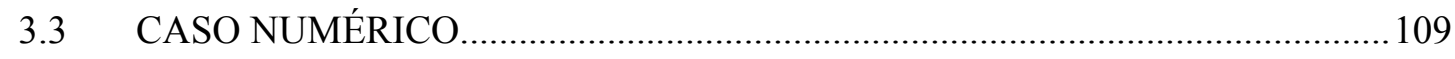

3.3.1 Parámetros generales del ejemplo numérico............................................. 110

3.3.2 Simulación numérica y modelo gráfico .................................................... 111

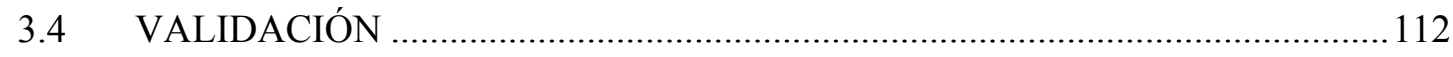

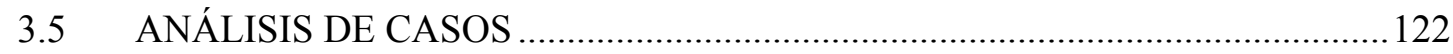

3.5.1 Caso A - Eje principal mayor próximo al eje de giro .................................... 125

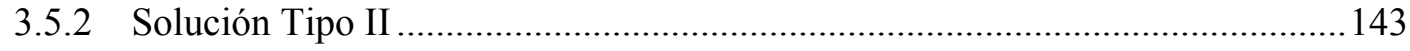

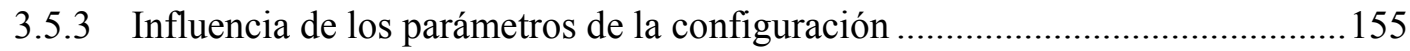

3.5.4 Caso B - Eje principal intermedio próximo al eje de giro. $I_{2}>I_{3}>I_{1} \ldots \ldots \ldots \ldots \ldots 174$

3.5.5 Caso C - Eje principal intermedio próximo al eje de giro. $I_{1}>I_{3}>I_{2} \ldots \ldots \ldots \ldots \ldots 177$

3.5.6 Caso D - Eje principal menor próximo al eje de giro. .................................... 179

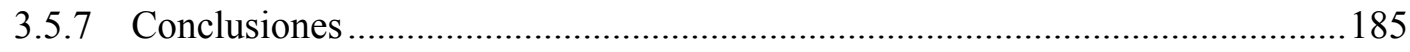

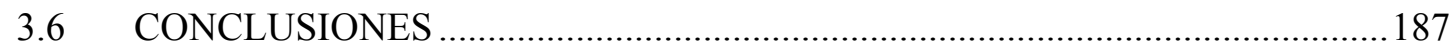

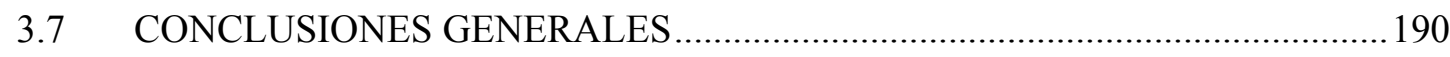

CAPÍTULO 4

ESTUDIO EXPERIMENTAL DEL EFECTO DEL DESPLAZAMIENTO DEL PLANO QUE CONTIENE A LAS PALAS CON RESPECTO AL CENTRO DE MASAS EN LA 
DINÁMICA DEL VUELO DE UN DESACELERADOR AERODINÁMICO DE ALAS ROTATORIAS

4.1 INTRODUCCIÓN

4.2 ENSAYOS EN TÚNEL AERODINÁMICO VERTICAL SOBRE UN MODELO EN VUELO LIBRE

4.2.1 Descripción del modelo físico y configuración experimental

4.2.2 Parámetros medidos e instrumentación

4.2.3 Definiciones .

4.2.4 Ensayos realizados y resultados 198

4.2.5 Análisis de los resultados .204

4.2.6 Incertidumbre de las mediciones .209

4.3 ENSAYOS EN TÚNEL AERODINÁMICO SOBRE UN MODELO QUE ROTA

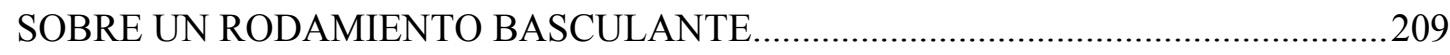

4.3.1 Descripción del modelo y configuración experimental ..................................209

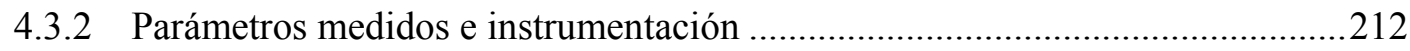

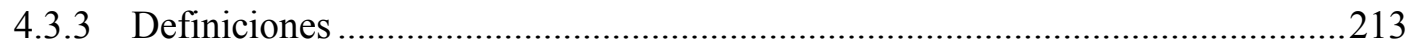

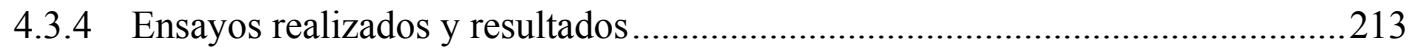

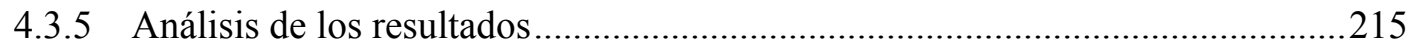

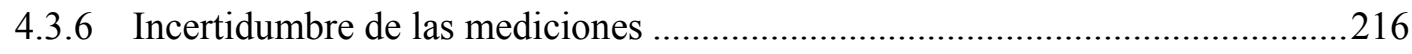

4.4 ENSAYOS REALIZADOS EN TRABAJOS DE REFERENCIA .......................216

4.4.1 Descripción del modelo y configuración experimental ................................217 
4.4.2 Parámetros medidos e instrumentación ..........................................................2. 218

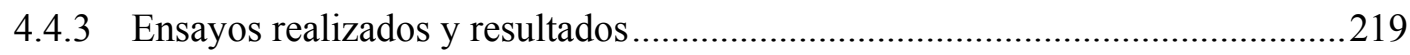

4.4.4 Comparativa con la simulación numérica y resultados..................................2219

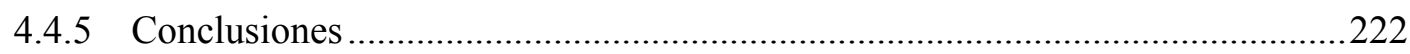

4.5 COMPARACIÓN DE LOS RESULTADOS DE LOS DIFERENTES ENSAYOS...

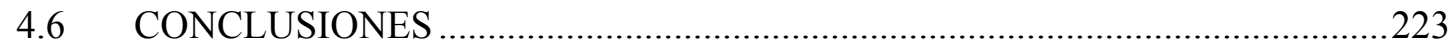

\section{CAPÍTULO 5}

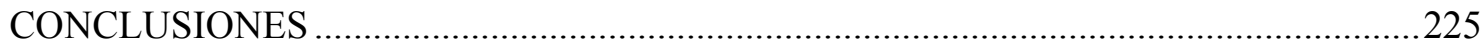

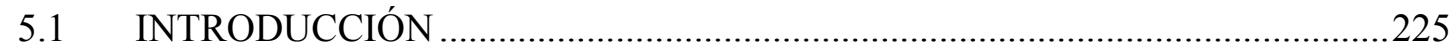

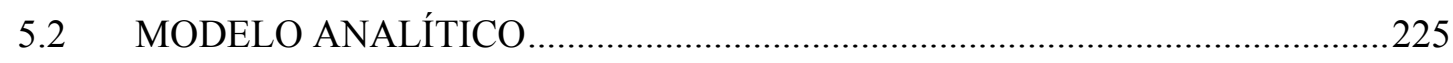

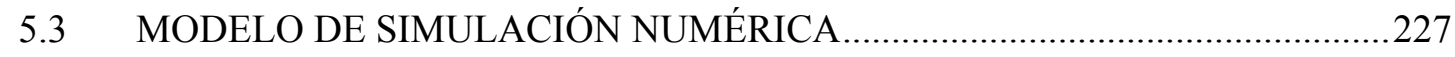

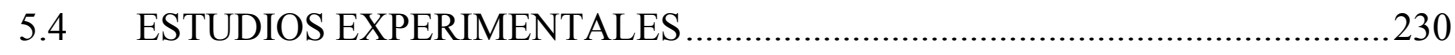

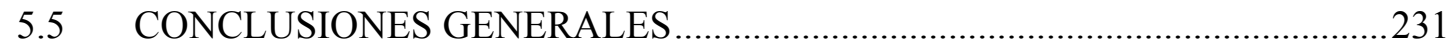

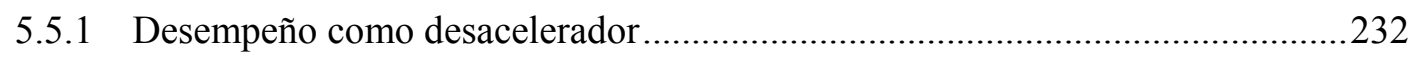

5.5.2 Comportamiento dinámico y estabilidad del pararrotor .................................233

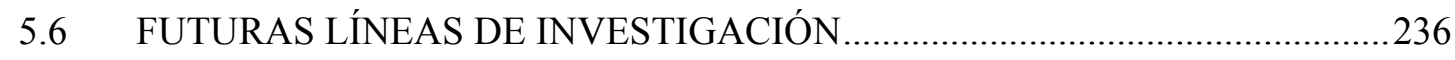

5.7 APORTES ORIGINALES DE LA PRESENTE TESIS ....................................236

ANEXO 1 
ESTUDIO ACERCA DE LOS COEFICIENTES AERODINÁMICOS DE PLACAS DE BAJO ALARGAMIENTO

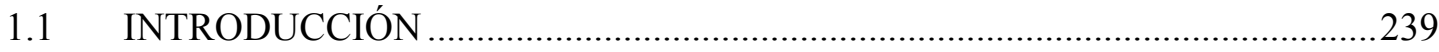

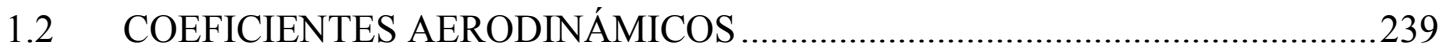

1.1.1 Modelo del coeficiente de sustentación .....................................................239

1.1.1 Modelo del coeficiente de resistencia .........................................................242

1.1.1 Variación de $C_{L \alpha} / C_{D}$ con el ángulo de ataque............................................243

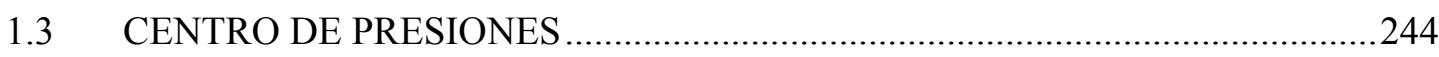

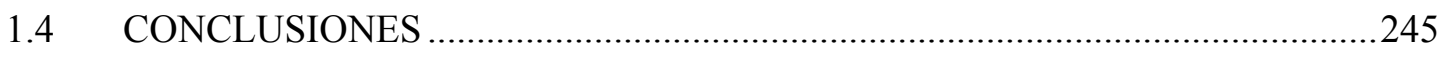

ANEXO 2

RESULTADOS EXPERIMENTALES

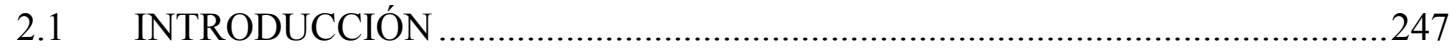

2.2 ENSAYOS EN TÚNEL AERODINÁMICO VERTICAL SOBRE UN MODELO EN VUELO LIBRE

2.3 ENSAYOS EN TÚNEL AERODINÁMICO SOBRE UN MODELO QUE ROTA

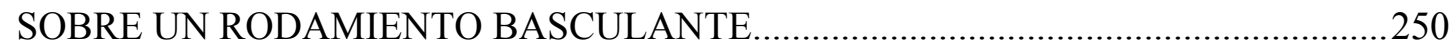

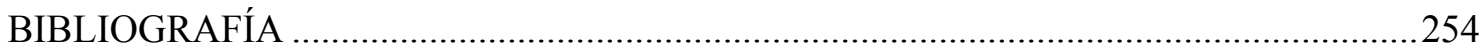




\section{NOMENCLATURA}

A, matriz de coeficientes de la linealización del sistema de ecuaciones del movimiento,

$A_{i j}, \quad$ coeficientes de fila $i$ y columna $j$ de la matriz $A$,

$b, \quad$ envergadura de la pala del pararrotor,

$B_{i}, \quad$ matriz de coeficientes constantes para una función de la $i$-ésima variable,

c, cuerda de la pala del pararrotor,

$C_{D}, \quad$ coeficiente de resistencia aerodinámica de la pala,

$C_{D 0}, \quad$ coeficiente de resistencia parásita,

$c_{D M}, \quad$ coeficiente de resistencia del modelo,

$C_{M}, \quad$ centro de masas del pararrotor,

$\boldsymbol{C}_{m i}$, componente $i$ de momentos adimensionalizados,

$C_{L \alpha}, \quad$ pendiente de la curva de sustentación de la pala,

$C_{L \alpha \text { no lineal }}, \quad$ componente no lineal del coeficiente de sustentación de la pala ,

$C_{\text {La lineal }}, \quad$ componente lineal del coeficiente de sustentación de la pala,

$C_{p}(x)$, distribución de carga en dirección de la cuerda

$C_{P i}, \quad$ centro de presiones en ejes cuerpo de la pala $i$,

$\operatorname{DCM}(\mathbf{q})$, matriz de cosenos directores en función del cuaternión de orientación $\boldsymbol{q}$,

Det, determinante de la matriz característica,

$\overline{D_{i}}, \quad$ resistencia generada por la pala $i$, 
$D_{i j}, \quad$ componente $j$ de la resistencia de la pala $i$,

$\mathbf{e}_{D i}, \quad$ versor en la dirección de la resistencia de la pala $i$,

$\boldsymbol{E}_{i}, \quad$ Matrices de coeficientes para la función diferencial de la variable $x_{2}$ en el i-ésimo lugar,

$\mathbf{e}_{i}, \quad$ vectores de orientación según el eje $i$ del sistema de ejes cuerpo,

$\mathbf{e}_{L i}, \quad$ versor en la dirección de la sustentación de la pala $i$,

$E_{N}, \quad$ error de la solución del modelo de simulación de la dinámica del pararrotor al respecto de la solución analítica,

Er, error relativo,

$E u l_{0}, \quad$ conjunto de los valores iniciales de los ángulos de Euler del pararrotor,

$\mathbf{F}_{D i}, \quad$ fuerza de resistencia generada por la pala $i$,

$F_{D i j}, \quad$ componente $j$ de la resistencia de la pala $i$,

$\mathbf{F}_{L i}, \quad$ fuerza de sustentación sobre la pala $i$,

$F_{L i j}, \quad$ componente $j$ de la sustentación de la pala $i$,

h, paso temporal del proceso de integración numérica,

$I_{i}, \quad$ momentos principales de inercia,

k' coeficiente empírico de la componente no lineal del coeficiente de sustentación,

$\overline{L_{i}}, \quad$ fuerza de sustentación sobre la pala $i$,

$L_{i j}, \quad$ componente $j$ de la sustentación de la pala $i$,

$k$, relación de velocidades,

$k_{e}$, relación de momentos de inercia, 
$k_{21}, \quad$ relación $r_{21} / r_{11}$,

$k_{31}, \quad$ relación $r_{31} / r_{11}$,

$K_{\varepsilon}, \quad$ ganancia de normalización,

$K_{i}, \quad$ constante de relación de inercia,

$N_{e}$, Número de Estabilidad,

$M_{i}, \quad$ componentes de los momentos de las fuerzas exteriores en la dirección $i$,

M, momento generado por las fuerzas exteriores sobre el cuerpo,

n, parámetro de distribución de carga en la cuerda en función del alargamiento del ala

$\mathbf{n}_{i}, \quad$ matriz de transformación para la obtención del módulo de la proyección de un vector sobre la dirección perpendicular a la pala $i$,

p, paso de integración numérica,

q. cuaternión que representa la orientación de la terna móvil al respecto de la fija,

$q_{i}$ componentes del cuaternión que representa la orientación de la terna móvil al respecto de la fija,

$R$, radio de la punta de pala en el modelo,

Re, número de Reynolds,

$r_{i j}$, componente $j$ de la posición de la pala $i$ al respecto del centro de masas del cuerpo,

$R_{60 \%}$ radio al $60 \%$ de la envergadura de la pala,

$S$, área de una sola pala,

$S_{p}, \quad$ área de ambas palas,

$T$, tracción, fuerza resultante en dirección del eje $Z$ inercial, 
$t_{e e}$ tiempo de establecimiento de la solución de equilibrio,

Tr, traza de la matriz característica,

$U_{\infty}, \quad$ velocidad del pararrotor en dirección al eje vertical inercial,

$V_{n i}, \quad$ velocidad normal a la pala $i$,

$\mathbf{V}_{b i}, \quad$ velocidad de translación en coordenadas del cuerpo,

$\mathbf{V}_{e}, \quad$ velocidad en coordenadas inerciales,

$\mathbf{V}_{r i}, \quad$ velocidad relativa de la pala $i$,

$V_{t}$, velocidad tangencial,

$\mathbf{V}_{t c}$, velocidad del pararrotor con respecto a los ejes inerciales expresado en coordenadas del cuerpo,

$\mathbf{V}_{v}, \quad$ velocidad vertical,

$x$ coordenadas en la dirección de la cuerda, con origen en el borde de ataque,

$x_{e q i}, \quad$ variable $i$ del sistema de ecuaciones de movimiento adimensionalizadas, $\omega_{i} / \omega_{0}$, para el equilibrio.

$x_{i}, \quad$ variable $i$ del sistema de ecuaciones de movimiento adimensionalizadas, $\omega_{i} / \omega_{0}$,

$x_{p \%}, \quad$ posición en porcentaje de cuerda del centro de presiones de la pala

$X, \quad$ eje inercial de la terna $X, Y, Z$,

$\mathbf{X}_{e}, \quad$ posición del pararrotor en coordenadas inerciales,

$Y$, eje inercial de la terna $X, Y, Z$,

$Z$, $\quad$ eje inercial de la terna $X, Y, Z$,

$\alpha_{i}, \quad$ ángulo de ataque de la pala $i$, 
$\beta_{i}, \quad$ ángulo de paso de las pala $i$,

$\delta_{\beta}, \quad$ diferencia de paso entre las palas,

$\Delta$, discriminante de la matriz característica,

1, relación de alargamiento de las palas,

$\varepsilon, \quad$ ángulo dado por la suma algebraica del paso de las palas,

$\varepsilon$, error de ortonormalidad,

$\lambda_{1,2}, \quad$ autovalores de la matriz $A$,

$\lambda_{A i}, \quad$ primer término del autovalor $i$ de la matriz $A$,

$\lambda_{B i}, \quad$ segundo término del autovalor $i$ de la matriz $A$,

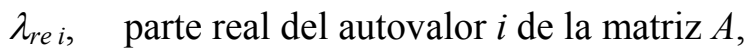

$\lambda_{\text {im } i,}$ parte imaginaria del autovalor $i$ de la matriz $A$,

$\theta$ ángulo de nutación,

$\theta_{e}$, ángulo de nutación de equilibrio,

$\rho, \quad$ densidad del aire,

$\varphi$, ángulo de rotación propia del cuerpo,

$\psi$, ángulo de precesión del cuerpo,

v, viscosidad cinemática,

$\omega_{12}, \quad$ velocidad angular compuesta por $\omega_{1}$ y $\omega_{2}$

$\omega_{i}, \quad$ velocidad angular en dirección del eje $i$ en coordenadas ejes cuerpo,

$\omega_{0}, \quad$ velocidad angular de referencia. 


\section{RESUMEN}

El objeto de la presente tesis es el estudio del comportamiento dinámico de un tipo de desacelerador aerodinámico basado en el concepto de pararrotor.

Surge del estudio de antecedentes que el concepto de decelerador es de origen biomimético, y que ha sido estudiado desde distintas ópticas, que incluye desde estudios de base biológica hasta aplicaciones específicas de base tecnológica, para su utilización práctica con fines diversos. Entre estos se destaca la recuperación de elementos provenientes del espacio, el control de la trayectoria de proyectiles, las sondas meteorológicas para mediciones medioambientales, la entrada de vehículos tripulados o no tripulados a determinada atmósfera, el guiado de dispositivos aerodinámicos en vuelo. No obstante es muy limitado el conocimiento que se tiene sobre la dinámica de los pararrotores, principal fuente de aporte al estado del arte de la presente tesis.

El pararrotor es un dispositivo desacelerador aerodinámico basado en la autorrotación de alas giratorias. La autorrotación es la rotación continua de un cuerpo inmerso en un flujo axial sin otras fuentes externas de energía más que la propia acción de la corriente de fluido y de la gravedad. Cuando el cuerpo se desplaza en un fluido, las palas rotan y, como consecuencia de este movimiento, se generan fuerzas aerodinámicas que actúan en oposición a su movimiento de translación principal. Su desempeño y utilización práctica está supeditada al conocimiento de su dinámica del vuelo en función de los parámetros aerodinámicos y geométricos del dispositivo.

El desarrollo de la presente tesis comprende el desarrollo de un modelo analítico simplificado del comportamiento dinámico del dispositivo, el desarrollo de una rutina de simulación numérica, y la realización de ensayos en túnel vertical y horizontal, con el fin de establecer los efectos de los parámetros de configuración del pararrotor sobre su comportamiento dinámico, y más específicamente sobre su estabilidad. Por otro lado se estudian parámetros 
que puedan servir para tipificar y clasificar la respuesta dinámica de los pararrotores de manera general.

El desarrollo de un modelo analítico que se aborda en el presente trabajo complementa los estudios realizados por Nadal Mora (2005) sobre la estabilidad del comportamiento dinámico del pararrotor. En estos estudios se caracteriza la estabilidad del pararrotor teniendo en cuenta una serie de simplificaciones que permiten acceder a expresiones útiles para el diseño general de estos dispositivos, teniendo en cuenta el desplazamiento del centro de masas con respecto al plano de las palas.

La rutina de simulación numérica adoptada para el estudio del efecto del desplazamiento del plano que contiene a las palas al respecto del centro de masas en un pararrotor se basa en las ecuaciones de la dinámica del pararrotor en su formulación completa. Sobre la simulación de casos específicos se estudia la estabilidad dinámica del sistema considerando el efecto que resulta del desplazamiento del centro de masas al respecto del plano que contiene a las palas en conjunto con parámetros geométricos y aerodinámicos.

Para el estudio experimental del pararrotor se prepararon una serie de ensayos sobre la base de modelos físicos del dispositivo para realizar investigaciones experimentales realizadas en el tema, a saber:

- Ensayos en túnel aerodinámico vertical sobre un modelo en vuelo libre.

- Ensayos en túnel aerodinámico sobre un modelo que rota sobre un rodamiento basculante.

El objeto de los ensayos experimentales es caracterizar el comportamiento dinámico del pararrotor a través de ensayos experimentales, útiles para disponer de elementos para validar los modelos teóricos y el simulador numérico.

Las investigaciones realizadas concluyen que es posible obtener 3 tipos de soluciones, dos estables y una inestable. El aumento de la distancia entre el plano que contine a las palas y el centro de masas condicionan fuertemente el tipo de solución que es posible alcanzar.

A través de la variación de una serie de parámetros geométricos y aerodinámicos es posible predecir y eventualmente controlar el comportamiento dinámico del pararrotor. En particular 
el tiempo de respuesta del sistema a una perturbación y la solución de equilibrio que determina la actitud del pararrotor son dependientes de estos parámetros.

\section{CAPÍTULO 1}

\section{INTRODUCCIÓN}

\subsection{INTRODUCCIÓN AL CONCEPTO DE PARARROTOR}

La presente Tesis se inicia con una introducción al concepto, funciones y características de los deceleradores aerodinámicos en general y del pararrotor en particular. En esta introducción se explica la necesidad de frenar la caída de una sonda en la atmósfera, se mencionan posibles aplicaciones de estos dispositivos y los sistemas existentes para la medición de dichos parámetros, se analizan los antecedentes en el estudio del pararrotor, se explica el fenómeno de la autorrotación y se presenta el concepto de pararrotor a estudiar.

\subsection{ANTECEDENTES}

El presente trabajo aborda el estudio de un tipo particular de desacelerador de la velocidad de caída de un cuerpo en presencia de atmósfera. Existen varios mecanismos para decelerar cuerpos en caída en la atmósfera, que se podrían agrupar en aerodinámicos, y no aerodinámicos. Dentro de los primeros, se encuentran los paracaídas $\mathrm{y}$, los denominados pararrotores, entre otros. En el segundo grupo, podrían ubicarse los sistemas con propulsión propia, los airbags, etc. Existen por otro lado desaceleradores que combinan mecanismos aerodinámicos y no aerodinámicos como los globos. Entre las características más significativas de un desacelerador aerodinámico se encuentra su capacidad de producir resistencia aerodinámica la cual es utilizada para limitar la velocidad del cuerpo. Se conoce como pararrotor a un ala giratoria en el régimen de autorrotación. 
El concepto de usar alas rotantes para la deceleración aerodinámica es una idea de origen biomimético que ha empezado a ser estudiado hace varias décadas. Se ha encontrado a través de las investigaciones realizadas, por medio de modelos teóricos y ensayos, que los pararrotores presentan desafíos en cuanto a su controlabilidad, principalmente debido a la gran no linealidad de su comportamiento.

Se han realizado numerosos estudios tendientes a hallar dispositivos aerodinámicos para frenar y controlar la caída de cuerpos en la atmósfera. Quizás el más conocido y estudiado es el paracaídas. No obstante, esta solución presenta algunas limitaciones que hacen necesario estudiar posibles alternativas. Algunos métodos alternativos consisten en la utilización de aletas o asimetrías para generar rotación, y a su vez sustentación, y de esta forma incrementar la resistencia aerodinámica de los cuerpos, y a veces también la estabilidad del movimiento. Algunas de estas investigaciones se han orientado al guiado de proyectiles, a la recuperación de vehículos de re-entrada en la atmósfera y a las mediciones de parámetros atmosféricos.

\subsubsection{Antecedentes en investigaciones sobre casos biológicos}

En la naturaleza, los deceleradores son usados para dispersar semillas a distancias suficientemente apartadas de su punto de lanzamiento (árbol padre), de manera que los nuevos especímenes no compitan con sus padres en la obtención de sus fuentes de subsistencia (agua, luz, nutrientes, etc.). Los deceleradores biológicos se pueden dividir en seis tipos básicos: (a) de descenso vertical, (b) de planeo recto, (c) de planeo espiral, (d) de planeo en zigzag, (e) rotantes alrededor de su eje principal, (f) rotantes alrededor de dos ejes (Shizuka Minami, 2003).

La estabilidad dinámica es también importante en la función biológica de dispersión de las semillas. Los deceleradores biológicos de tipo rotatorio alrededor de su eje principal (pararrotores) suelen tener gran estabilidad dinámica y por ende son más robustos para tolerar perturbaciones que provengan de la turbulencia atmosférica (McCutchen, 1977).

Las características más importantes para la clasificación, descripción funcional y utilización de los pararrotores son la estabilidad dinámica de su vuelo, y capacidad de regular su velocidad de descenso. 
Entre las geometrías que pueden encontrarse en la naturaleza se encuentra la denominada Sámara que es descripta por la hoja del arce. En este caso la carga se encuentra localizada en el extremo del ala. El ala rota alrededor de un eje cercano su centro de masa. El vuelo de la semilla puede describirse con un patrón helicoidal.

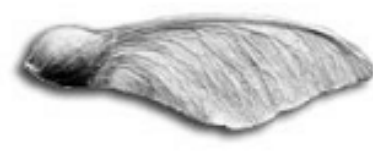

(a)

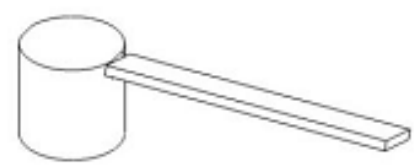

(b)

Figura 0.1 Configuración de pararrotores tipo Sámaras. En (a) se muestra una semilla de arce, en (b) un esquema simplificado.

Se han investigado diversas especies de Sámaras, clasificándolas por su desempeño como desacelerador, tomando en cuenta velocidad de descenso, ángulo de inclinación sobre el eje vertical y velocidad de rotación en equilibrio dinámico. No obstante no se han desarrollado modelos aerodinámicos completos a pesar de la existencia de estudios de correlación entre parámetros realizados, entre otros, por Greene (1993), Horn (2001), Kunio Yasuda (1997), Matlack (1987).

Cuando un pararrotor se lanza, el paso de las palas se ajusta de manera de alcanzar un equilibrio entre la distribución geométrica de las palas y el ángulo de cono que forma el eje principal adherido al cuerpo y el eje vertical (Walker, 1981). La habilidad de un pararrotor en la naturaleza para automáticamente estabilizarse de distintas condiciones iniciales es crítica para el éxito del objeto biológico del dispositivo.

Rosen y Seter (1991) desarrollaron un modelo con el objeto de predecir el equilibrio dinámico de un pararrotor tipo Sámara. El modelo se basa en la teoría del Elemento de Pala. Los resultados obtenidos fueron comparados con las observaciones publicadas en publicaciones sobre biología que convalidan el modelo a pesar de las fuentes importantes de incertidumbre en las mediciones (A. Rosen, 1991). En el trabajo publicado en el año 1992, 
Seter y Rosen presentan un modelo numérico para investigar la estabilidad de la autorrotación vertical de la sámara, obtenido a partir del método de pequeñas perturbaciones aplicado a las ecuaciones del movimiento de la sámara. Estudian la influencia de diferentes parámetros en la estabilidad. Comparan los resultados del modelo con resultados experimentales cualitativos realizados sobre sámaras artificiales diversas.

\subsubsection{Antecedentes en investigaciones sobre casos de aplicación}

El principio de la sámara, colocando una sola ala a un cuerpo, fue estudiado para aplicaciones en caída de municiones por Crimi (1988), mediante una simulación numérica. Este autor sostiene que su dispositivo permite alcanzar buena efectividad en la caída de la submunición debido al régimen de descenso constante y al movimiento de rotación periódico. Agrega que el cuerpo se mueve con un movimiento donde el ángulo entre el eje vertical del sistema inercial y el eje de rotación propia del cuerpo es constante, y la velocidad de precesión es constante e igual a la velocidad de rotación del cuerpo. El análisis involucra a 11 variables para describir el movimiento del cuerpo en régimen estacionario. Realiza adicionalmente una comparación con resultados experimentales para varias configuraciones diferentes; compara el ángulo de conicidad, la velocidad propia de giro y la velocidad de caída del cuerpo.

Karlsen, Borgström, y Paulsson (1991), describen la aerodinámica de un cuerpo giratorio provisto de aletas, para ser utilizado como submunición de artillería. Comentan las desventajas de un paracaídas rotatorio respecto de un sistema giratorio con aletas rígidas: las dificultades para desplegar el paracaídas apropiadamente, los amplios movimientos de precesión de los sistemas con paracaídas, la funcionalidad de un paracaídas para decelerar una carga, etc. Por otra parte, la mayor velocidad de descenso del sistema de alas rígidas es beneficiosa para esta aplicación. También mencionan la mayor fiabilidad de los ensayos en túnel aerodinámico de un sistema con aletas rígidas respecto de uno con superficies flexibles. Señalan que la sensibilidad a los vientos de un sistema rígido es mucho menor que la de los paracaídas. Indican que la relación ente la velocidad de caída, la velocidad de giro y el ángulo de ataque es muy importante para la orientación del cuerpo en el espacio. También que la relación entre el momento de inercia respecto al eje principal de rotación, $I_{3}$, y los momentos de inercia respecto a los otros dos ejes del sistema de referencia fijo al cuerpo, $I_{2}$ y $I_{1}$, debe 
ser alrededor de 1,1 para obtener un movimiento rápido en la orientación del cuerpo y un amortiguamiento suficiente en los movimientos de precesión.

Pepper Jr. (1986), presenta un diseño de paracaídas giratorio de altas prestaciones, aplicable a la recuperación de vehículos en re-entrada a la atmósfera. Destaca la ventaja del vuelo en autorrotación, en particular, con referencia a la mayor estabilidad giroscópica y resistencia aerodinámica que se obtienen.

Por otro lado Crimi (1996) desarrolló modelos analíticos de pararrotores. La conclusión de distintos experimentos, desarrollos analíticos y simulaciones es que existen múltiples soluciones estables. A través de las distintas investigaciones se demuestra que la dinámica del pararrotor es altamente no lineal. Se utilizaron en general técnicas computacionales para el cálculo de condiciones de equilibrio. No se han presentado análisis de la respuesta en el tiempo de la dinámica de los pararrotores.

Kellas ha estudiado la configuración tipo sámara para la generación de un modelo de dinámica controlada.

También Doherr y Schilling (1992) han estudiado sistemas de paracaídas giratorios, para decelerar municiones. En la referencia mencionada realizan una simulación numérica de la trayectoria y la dinámica del sistema de paracaídas rotatorio. Comentan que los paracaídas rotatorios son muy sensibles a las perturbaciones atmosféricas.

Las fuerzas Armadas de Estados Unidos han realizado investigaciones al respecto del uso de deceleradores del tipo Sámara para el lanzamiento de municiones de manera que permita la medición de parámetros del blanco en el descenso de la munición (Pillasch, 1993).

Shpund y Levin han enfocado su estudio a las propiedades aerodinámicas de paracaídas giratorios. En la referencia (Levin, Shpund, 1995) explican que el propósito de sus estudios es la aplicación a municiones que deben realizar maniobras complicadas mientras descienden en busca de blancos. El paracaídas se utiliza como mecanismo de control del vuelo. El objetivo es poder controlar el régimen de descenso, la velocidad de giro y la posición espacial instantánea de la carga. El estudio de la referencia (Levin, Shpund, 1995) está orientado a entender los efectos de las variables geométricas del paracaídas (relación entre el ancho y el 
largo de las bandas del paracaídas -relación de aspecto, porosidad geométrica-, y relación entre la longitud de las bandas y la longitud de las cuerdas de suspensión) en la estabilidad dinámica del sistema. Estudian paracaídas tipo “cruz". Muestran que los resultados presentan movimientos estables e inestables, dependiendo de las variables mencionadas; a mayor relación de aspecto se alcanza mayor estabilidad. La relación entre la longitud de las bandas y la longitud de las cuerdas de suspensión resulta en diferentes efectos sobre la estabilidad, dependiendo de la velocidad de rotación propia: a bajas velocidades de rotación propia la estabilidad aumenta con el aumento de la relación mencionada, mientras que a altas velocidades de rotación propia sucede lo opuesto. Obtienen una configuración estable donde el sistema presenta movimiento de precesión, describiendo un movimiento cónico estable y donde la frecuencia dominante es el giro sobre el propio eje. Destacan la presencia de tres frecuencias dominantes: las dos más bajas relacionadas con las propiedades de inercia y aerodinámicas de la carga y del paracaídas; la tercera relacionada con la velocidad de giro propia, y depende estrechamente de las variables geométricas (las dos primeras varían poco con los cambios en la configuración). Exponen también las diferencias que existen entre los ensayos en túnel aerodinámico, donde el movimiento del modelo está restringido, y el vuelo libre. Afirman que, sin embargo, las principales propiedades aerodinámicas, estáticas y dinámicas, y las propiedades de inercia se manifiestan, en ambos casos, de forma cualitativamente igual aunque no cuantitativamente y que los resultados de los ensayos en túnel aerodinámico podrían ser empleados para el diseño y análisis de las configuraciones reales. En la referencia (Shpund, Levin, 1997), los mismos autores investigan la interferencia aerodinámica entre la carga útil y el paracaídas, para sistemas giratorios y no giratorios. En la referencia (Levin, Shpund, 1997), los autores estudian el efecto de la geometría del paracaídas. Mencionan el aumento de la resistencia aerodinámica que se genera en los paracaídas por el movimiento de rotación y la intención de que el paracaídas sirva como medio de control de la trayectoria del sistema paracaídas más carga.

En la década de 1960 y 1970 la NASA investigó un concepto de vehículo de entrada planetaria basado en el concepto de pararrotor. Este diseño daba a los astronautas la habilidad de maniobrar y aterrizar el vehículo de manera precisa y segura. Por diversos problemas asociados con los regímenes de descenso, madurez de la tecnología, capacidad de control, se supone que el concepto se abandonó. 


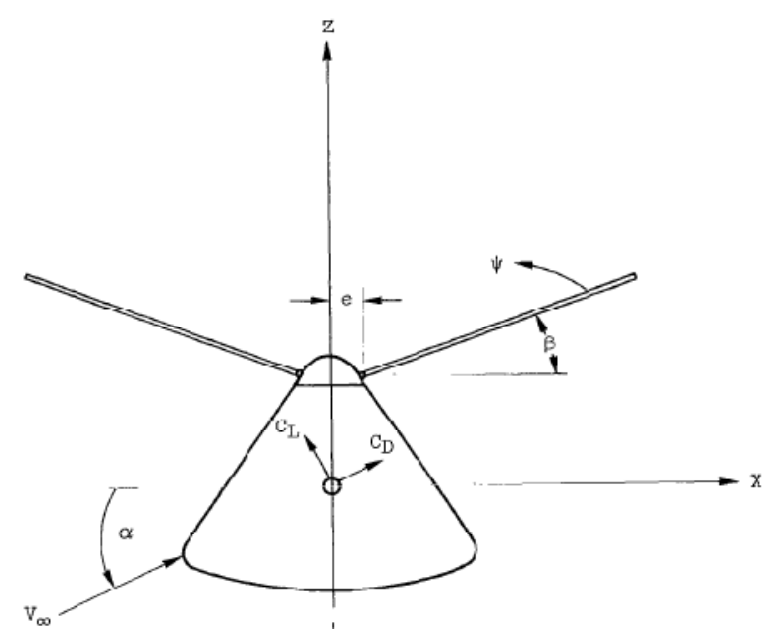

Figura 0.2 Configuración de vehículo de entrada planetaria.

En la década de 1990 se desarrolló de forma privada otro tipo de concepto de vehículo basado en el pararrotor que resulta de la introducción de palas a un cohete para su recuperación después de cumplida su función de posicionamiento orbital. Un ejemplo de este tipo es el concebido por McKinney (1998). Prototipos de este tipo fueron ensayados en vuelo con éxito. Aparentemente por problemas comerciales se abandonó la empresa.

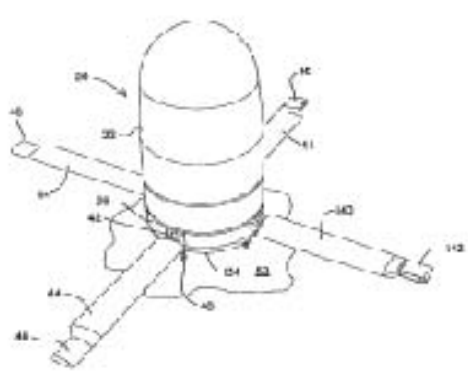

(a)

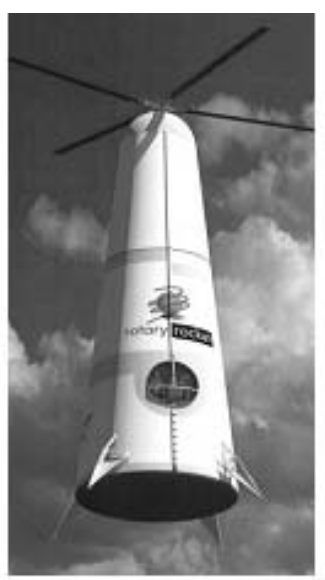

(b)

Figura 0.3 Cohetes asimilables al concepto de pararrotor (a) concepto original (McKinney, 1998), (b) modelo de prueba. 
Otro concepto de vehículo de entrada planetaria fue desarrollado para ser incorporada en el denominado Crew Exploration Vehicle (CEV), potencial reemplazo del Space Shuttle propiedad de NASA, para el descenso final del vehículo explorador. Este concepto incluía una serie de tres palas plegables.

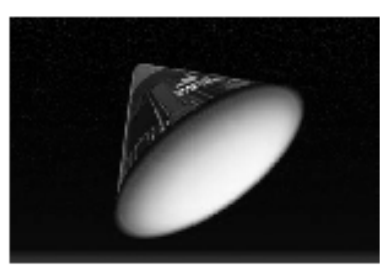

(a)

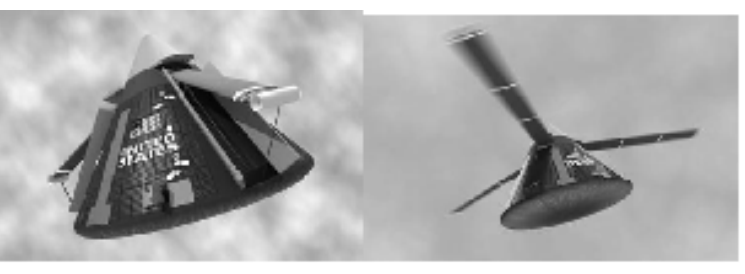

(b) (c)

Figura 0.4 Secuencia conceptual de entrada, descenso y aterrizaje para un pararrotor $\mathrm{CEV}$, donde (a) entrada, (b) despliegue del rotor, (3) descenso en autorrotación. Hagen (2005).

Finalmente, otro tipo de aplicación del concepto de pararrotor son las sondas no tripuladas, militares o civiles. Este tipo de aplicación incluye ejemplos como:

- Sondas de medición atmosférica en aeropuertos con palas de pequeño alargamiento. Nadal Mora (2005).

- Sondas para la entrega de carga a unidades terrestres, impulsada por United States Naval Academy. Bartz \& Miklosovic, (2003).

- Microsondas de entrada planetaria. 


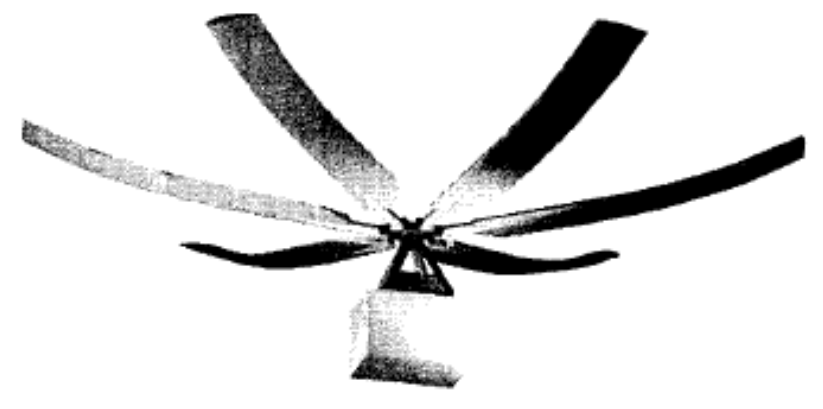

Figura 0.5 Desacelerador basado en el concepto de pararrotor para la entrega de carga a unidades terrestres.

En la actualidad la fuente de mayor interés en el uso de los pararrotores es el de sondas de entrada planetaria muy pequeñas, múltiples, de bajo costo (Cy Klein, 2006; Young, 2004; Dorrington 2011). El objeto conceptual de los diseños que se impulsan es la sencillez, alta confiabilidad, facilidad de despliegue $\mathrm{y}$, pequeño tamaño y costo. Existen actualmente numerosos esfuerzos en realizar desarrollos en este sentido. Un ejemplo de esta política de diseño de dispositivos de entrada podemos mencionar el Deep Space II Microprobe (Suzanne Smrekar, 1999). Esta microsonda de entrada no incluye un sistema de deceleración del tipo paracaídas ni ningún otro para evita agregarle carga. El Deep Space II Microprobe, de $3 \mathrm{~kg}$ de masa, fue diseñado para soportar el impacto con la superficie de Marte con la única deceleración de la Resistencia de su cuerpo. No obstante ninguna de las dos sondas fue exitosa en establecer contacto después de impactar con la superficie marciana (Andrew J. Ball, 2007).

En el desarrollo de la tesis "Comportamiento aerodinámico de sondas atmosféricas en entornos aeroportuarios”, por Nadal Mora, dirigido por el Dr. Ángel Sanz Andrés (2005), se ha estudiado en forma teórica y experimental el descenso en autorrotación axial $\mathrm{y}$, teóricamente, la dinámica del vuelo de un cilindro provisto de aletas. Respecto a la primera línea de investigación, se ha obtenido una herramienta matemática para predecir el comportamiento de un pararrotor que funciona en un régimen de autorrotación axial, se ha determinado cuáles son los parámetros que afectan al comportamiento del pararrotor y de qué 
manera se manifiesta su influencia, entre otros. Respecto al análisis de la dinámica del vuelo, se ha hallado un modelo que describe regiones estables e inestables, límites de estabilidad y condiciones de estabilidad para el giro alrededor de un eje próximo a uno de los ejes principales de mayor, menor e intermedio valor del momento de inercia.

Jukubowski (1974) propuso un disco flexible, rotatorio, como un decelerador de reentrada atmosférica potencial, mostrando ventajas operacionales tales como la alta relación entre potencial de deceleración y peso, refrigeración efectiva y potencial para realizar maniobras. Se ensayó en un túnel supersónico a Mach 4, modelos construidos en base a un cono con múltiples álabes concéntricos dotados de un aro perimetral exterior, y conos perforados. Un ejemplo de estos modelos se muestra en la Figura 1-6. En el estudio de referencia se concluye que el coeficiente de resistencia global del dispositivo aumenta principalmente con el aumento de la relación entre área total del cono y área sólida del cono, denominada porosidad.
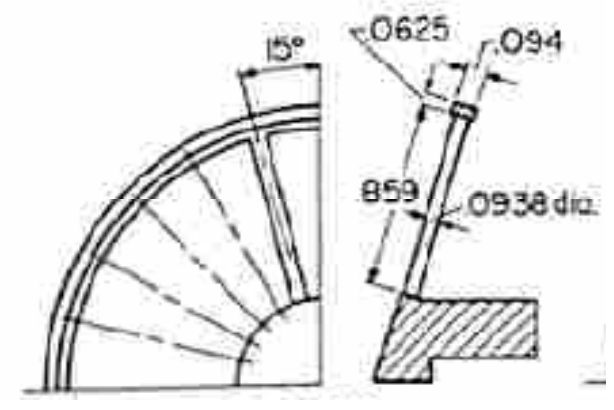

Figura 0.6 Geometría de un modelo de disco cónico ensayado por Jukubowski (1974).

Las cotas se encuentran expresadas en pulgadas.

\subsection{APLICACIONES DEL PARARROTOR}

Existen múltiples aplicaciones en las cuales se desea frenar la caída de un cuerpo en determinada atmósfera. Entre ellas se pueden mencionar:

- la recuperación de elementos provenientes del espacio,

- el control de la trayectoria de proyectiles, 
- las sondas meteorológicas para mediciones medioambientales,

- la entrada de vehículos tripulados o no tripulados a determinada atmósfera,

- el guiado de dispositivos aerodinámicos en vuelo.

En cada uno de estos casos existirá un alcance de aquello requerido de la deceleración, asociada a la misión del dispositivo. Algunas de las principales causas por las cuales es preciso frenar la caída de estos cuerpos es el aterrizaje en condiciones de deceleración controladas, el control de la trayectoria y el guiado, el control de la velocidad de descenso, el control de actitud.

En particular reviste de gran interés la utilización de este tipo de dispositivos para misiones autónomas en atmósferas no terrestres, donde la robustez, simplicidad y economía en el uso de energía resulta determinante. En este sentido se han desarrollado una enorme variedad de dispositivos con tecnología y misiones diversas a lo largo de la historia como muestra Ball y otros (2007), Steiner (2011), entre otros. En este sentido Ball y otros (2007) destaca la potencial utilización de dispositivos basados en la autorrotación como desacelerador en vehículos interplanetarios, concepto que no se ha aplicado aún. Se destaca en particular la posibilidad de la miniaturización y lo compacto del concepto.

Por otro lado se corrobora un importante interés en la industria armamentista asociado al concepto de pararrotor. Textron ha desarrollado un sistema de defensa basado en el concepto de sámara que, de acuerdo con lo que comunica la empresa, ha sido sumamente efectivo en operaciones militares recientes. Entre las características destacadas que menciona su página oficial se indica su bajo peso, su flexibilidad en el despliegue y su fácil integración. Este vehículo se describe como una munición autónoma capaz de seguir a su blanco una vez lanzado con un rango determinado de alcance. La Figura 1-7 muestra el dispositivo desarrollado por Textron. 


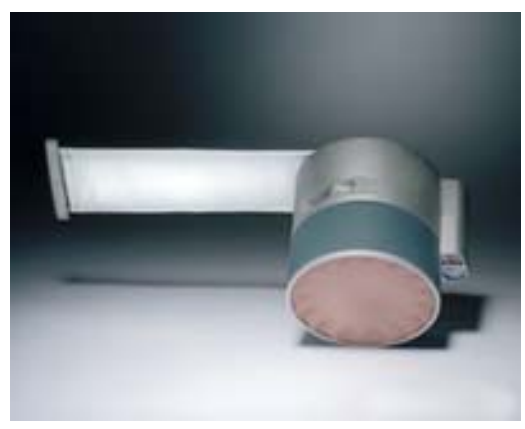

Figura 0.7 Desacelerador basado en el concepto de sámara para el guiado de municiones. Selectively targeted skeet. Textron Systems.

En resumen, como se ha mencionado, se pretende estudiar el frenado en la baja atmósfera por medios aerodinámicos de la caída de un cuerpo que transporta sondas de medición utilizando el principio del pararrotor.

\subsection{AUTORROTACIÓN}

La autorrotación es el fenómeno en el cual se basa el desempeño como desacelerador de los pararrotores. Resulta necesario hacer una breve reseña acerca de este fenómeno para sentar las bases sobre los cuales se desarrollarán las posteriores elaboraciones del presente trabajo. Por otro lado el contexto funcional del vuelo de los pararrotores se encuentra descripto por la autorrotación.

La autorrotación es la rotación continua de un cuerpo inmerso en un flujo axial sin otras fuentes externas de energía más que la propia acción de la corriente de fluido. Bajo esta definición, los molinos de viento, turbinas hidráulicas, anemómetros de cazoletas, frutos de especies vegetales tipo sámara, algunos tipos de extractores de aire, etc., funcionan bajo el principio de autorrotación. Todos estos dispositivos giran puramente por efecto de fuerzas fluidodinámicas, sin fuentes externas de energía; están conformados geométricamente de tal manera que, cuando se sitúan en el seno de una corriente de fluido, se crea un par que inicia la rotación cuando se libera el cuerpo permitiéndole girar libremente. En algunos casos, se requiere un impulso que inicia el movimiento. En los ejemplos mencionados se ve también 
que el eje de rotación del cuerpo puede ser paralelo o perpendicular a la dirección de la corriente; esto genera dos tipos de análisis diferentes (Lugt, 1983). Generalmente, los que poseen su eje de rotación perpendicular a la dirección de la corriente presentan movimientos periódicos, mientras que los de eje paralelo suelen presentar movimientos constantes.

También puede ocurrir que un cuerpo se mueva libremente en un fluido, generándose un movimiento de autorrotación que no sea perpendicular ni paralelo, sino que se mueva con seis grados de libertad, incluyendo efectos giroscópicos.

Aunque el régimen de autorrotación es ampliamente conocido (a pesar de lo cual todavía es objeto de investigación) por su aplicación al caso de los helicópteros, el problema del pararrotor estudiado aquí se diferencia fundamentalmente en el alargamiento de las palas, siendo en el presente caso de alargamiento pequeño, por lo que no son aplicables los conceptos desarrollados en el caso de helicópteros. Por razones de almacenamiento para su transporte, las palas del pararrotor deben ir plegadas sobre el cuerpo de la sonda, que suele ser cilíndrico, y cuya altura está limitada por la estabilidad giroscópica.

En el caso del pararrotor el mecanismo de autorrotación es el siguiente: si el pararrotor está inicialmente en reposo, al incidir una corriente sobre las palas se genera una fuerza aerodinámica que posee una componente tangencial que hace girar y acelerar el rotor. En un determinado momento, cuando se alcanza un régimen estacionario, la fuerza tangencial es cero y la resultante de las fuerzas aerodinámicas tiene la dirección del flujo incidente.

\subsection{MODELO DE PARARROTOR ESTUDIADO}

El pararrotor que se estudia en el presente trabajo tiene características que pretenden ser el punto de partida para el estudio de configuraciones particulares y estudios posteriores.

El modelo de pararrotor básicamente está compuesto por un cilindro dispuesto verticalmente el cual determina los ejes propios del cuerpo, que se denominarán 1,2 y 3 , siendo el eje 3 coincidente con la vertical del cilindro. Sobre el denominado eje 2 del cilindro se disponen dos palas cuyos ejes son coincidentes, alejadas una distancia determinada del eje del cilindro. Finalmente el pararrotor tiene un centro de masas que puede ser coincidente o no con el plano 
que contiene a las palas. Se consideran plantas alares de forma rectangular. Un ejemplo gráfico del modelo estudiado se presenta en la Figura 1.8.

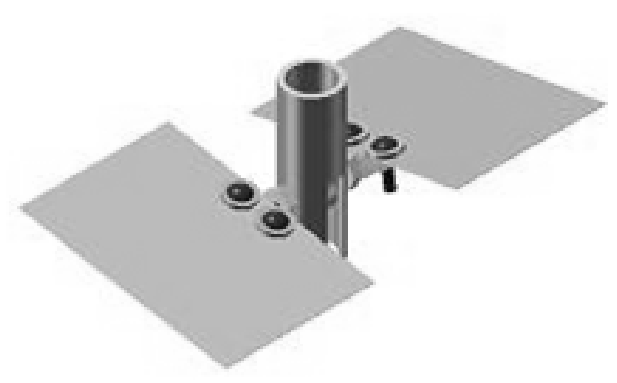

Figura 0.8 Representación típica del modelo de pararrotor estudiado.

A partir del estudio de los antecedentes de aplicación del concepto de pararrotor, conjuntamente con otros realizados por Sanz, Nadal Mora y Piechocki (2007), y Howard (2008), es posible observar que existen una serie de fortalezas que ameritan mayores esfuerzos en su estudio. Entre estas fortalezas se encuentra:

- es posible encontrar tanto situaciones de vuelo estables como inestables dependiendo de la configuración del pararrotor,

- es posible dotar al pararrotor de alas de pequeño alargamiento no sacrificando buen desempeño,

- el dispositivo se mantiene operativo incluso con desprendimiento de flujo en sus alas, y por tanto en un rango muy amplio de ángulos de ataque,

- permite la miniaturización de sondas atmosféricas y planetarias,

- permite configuraciones que pueden ser plegadas,

- permite el control y guiado del dispositivo por medio del control de su configuración. 


\subsection{CONTENIDO DE LA TESIS}

La Tesis se inicia con una introducción al concepto, funciones y características de los deceleradores aerodinámicos en general y del pararrotor en particular. En esta introducción se explica la necesidad de frenar la caída de una sonda en la atmósfera, se mencionan posibles aplicaciones de estos dispositivos y los sistemas existentes para las mediciones de dichos parámetros, se analizan los antecedentes en el estudio del pararrotor, y se explica el fenómeno de la autorrotación. También se introduce el modelo físico de parrarrotor que será estudiado. Finalmente se presenta el contenido de la tesis y sus objetivos.

En el Capítulo 2 se desarrolla un modelo teórico de la acción de las fuerzas aerodinámicas sobre el movimiento del cuerpo en rotación alrededor de un eje fijo, basado en aspectos de la teoría de cantidad de movimiento y la teoría de alas esbeltas. Este modelo permite predecir la variación con el ángulo de incidencia del flujo de la velocidad tangencial adimensional, la velocidad de la corriente adimensional, el ángulo de paso, el parámetro adimensional de relación de velocidades y el ángulo de ataque, para diferentes características aerodinámicas de las palas. El modelo permite identificar los factores de los que dependen los parámetros del funcionamiento del pararrotor y su influencia, y así poder explicar aspectos del comportamiento del pararrotor en los ensayos experimentales.

En el Capítulo 3 se desarrolla una rutina de simulación numérica a partir del modelo analítico desarrollado en forma completa que se describe en el capítulo anterior. En este capítulo por otro lado se valida la simulación a partir de soluciones conocidas. Finalmente se presentan una serie de casos para la apreciación de las soluciones de equilibrio frente a distintas variantes de los parámetros de configuración del pararrotor. En particular se observan los efectos del desplazamiento del plano que contiene a las palas al respecto del centro de masas.

En el Capítulo 4 se describen los ensayos experimentales realizados en relación con el modelo teórico desarrollado en el Capítulo 2, presentando el modelo físico utilizado, el instrumental, los procedimientos de ensayo, los parámetros medidos, las configuraciones ensayadas, etc. También se analizan los resultados considerando la reproducibilidad de los ensayos. Finalmente, se presentan las conclusiones relativas a los resultados de los ensayos experimentales. 
El Capítulo 5 se concluye acerca de los resultados de los capítulos 2, 3 y 4, que se derivan de su análisis comparativo.

Finalmente se incluye el Anexo 1, donde se estudia el efecto del bajo alargamiento de las palas del pararrotor sobre la aerodinámica del dispositivo, y el Anexo 2, donde se presentan los resultados experimentales. 


\section{CAPÍTULO 2}

\section{ESTUDIO TEÓRICO DEL EFECTO DEL DESPLAZAMIENTO DEL PLANO QUE CONTIENE A LAS PALAS CON RESPECTO AL CENTRO DE MASAS EN LA DINÁMICA DEL VUELO DE UN DESACELERADOR AERODINÁMICO DE ALAS ROTATORIAS}

\subsection{INTRODUCCIÓN}

El pararrotor es un dispositivo desacelerador aerodinámico basado en la autorrotación de alas giratorias. La autorrotación es la rotación continua de un cuerpo inmerso en un flujo axial sin otra fuente externa de energía más que la propia acción de la corriente de fluido y de la gravedad. Cuando el cuerpo se desplaza en un fluido, las palas rotan y, como consecuencia de este movimiento, se generan fuerzas aerodinámicas que actúan en oposición a su movimiento de translación principal. Su desempeño y utilización práctica está supeditada al conocimiento de su dinámica vuelo en función de los parámetros aerodinámicos y geométricos del dispositivo.

El problema que se aborda en el presente trabajo complementa los estudios realizados por Nadal Mora (2005) sobre la estabilidad del comportamiento dinámico del pararrotor. En estos estudios se caracteriza la estabilidad del pararrotor teniendo en cuenta una serie de simplificaciones que permiten acceder a expresiones útiles para el diseño general de estos dispositivos. En el presente trabajo se continúa dicho estudio, ampliándolo para tener en cuenta el efecto en la estabilidad del sistema del desplazamiento del centro de masas con respecto al plano de las palas.

La metodología adoptada consiste en plantear las ecuaciones de la dinámica del pararrotor en forma completa, para luego simplificarlas reteniendo los términos de orden mayor, y 
finalmente estudiar la estabilidad dinámica del sistema considerando el efecto que resulta del desplazamiento del centro de masas respecto del plano que contiene a las palas.

\subsection{MODELO MATEMÁTICO}

El sistema a estudiar corresponde a un dispositivo de vuelo rotatorio en régimen de autorrotación compuesto por un cuerpo cilíndrico con alas de pequeño alargamiento compuestas por placas planas, como se indica en la Figura 2.1.

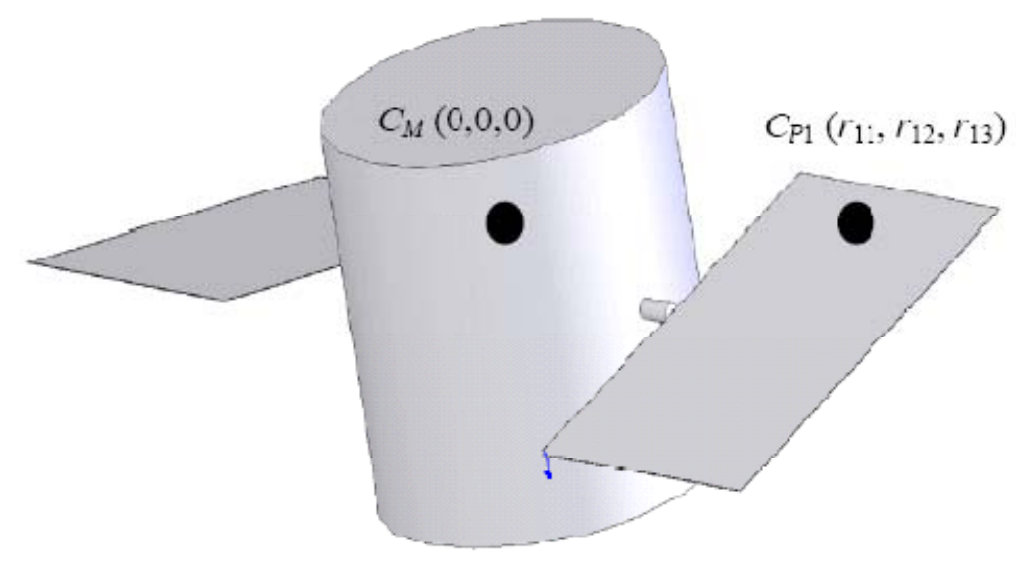

Figura 0.1 Esquema del pararrotor estudiado. Posición del centro de masas $\left(C_{M}\right)$ y posición del centro de presiones de la pala $1\left(C_{P 1}\right)$ en el sistema ejes cuerpo.

Considérese un cuerpo que cae a velocidad uniforme, $V_{t}$, según la vertical y girando con velocidad angular $\omega$. El sistema ejes cuerpo solidario al pararrotor, se designa por 1,2,3, con direcciones $\mathbf{e}_{1}, \mathbf{e}_{2}, \mathbf{e}_{3}$ y origen situado en el centro de masas. Estos son los ejes principales de inercia. El sistema de referencia inercial es $X, Y, Z$, cuyos ejes poseen las direcciones $\boldsymbol{i}, \boldsymbol{j}, \boldsymbol{k}$. La geometría del pararrotor está definida en la Figura 2.2. Es posible definir la orientación de la terna ejes cuerpo respecto de la inercial a partir de los ángulos de Euler. 


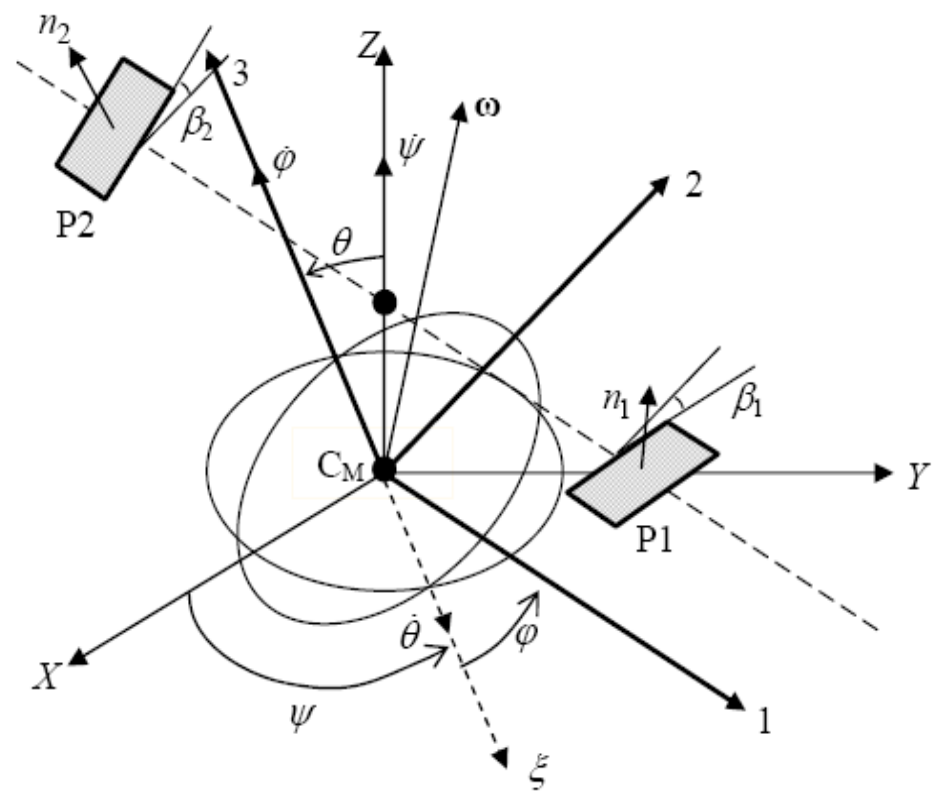

Figura 0.2 Definición de la geometría del pararrotor. $\psi$, precesión; $\theta$, nutación; $\varphi$, rotación propia.

El desarrollo matemático comprende:

- $\quad$ El planteamiento de las ecuaciones de la dinámica del pararrotor.

- El planteamiento de las expresiones de fuerzas y momentos aerodinámicos de forma completa, introduciendo el factor correspondiente al desplazamiento de centro de masas al respecto del plano formado por las palas.

- La comparación entre el nuevo sistema de ecuaciones y el obtenido por Nadal Mora (2005).

- $\quad$ El análisis de la estabilidad del nuevo sistema. 


\subsubsection{Ecuaciones de la dinámica del pararrotor}

Las ecuaciones de Euler de la dinámica rotacional del pararrotor en el sistema de referencia formado por los ejes principales de inercia pueden expresarse como:

$$
\begin{aligned}
& \frac{M_{1}}{I_{1}}=\frac{\mathrm{d} \omega_{1}}{\mathrm{~d} t}-\left(\frac{I_{2}-I_{3}}{I_{1}}\right) \omega_{3} \omega_{2}, \\
& \frac{M_{2}}{I_{2}}=\frac{\mathrm{d} \omega_{2}}{\mathrm{~d} t}+\left(\frac{I_{1}-I_{3}}{I_{2}}\right) \omega_{3} \omega_{1}, \\
& \frac{M_{3}}{I_{3}}=\frac{\mathrm{d} \omega_{3}}{\mathrm{~d} t}-\left(\frac{I_{1}-I_{2}}{I_{3}}\right) \omega_{2} \omega_{1} .
\end{aligned}
$$

\subsubsection{Ecuaciones de fuerzas y momentos}

A los fines de la elaboración del presente modelo se consideran una serie de simplificaciones, las cuales restringirán el problema a un determinado campo de aplicación dado: el movimiento de los ejes cuerpo será tal que siempre permanecerá próximo el eje 3 a la posición de la vertical inercial (eje $Z$ ).

Las componentes de la velocidad de caída en ejes cuerpo se obtienen mediante el producto de la matriz de rotación asociada a la transformación de Euler adoptada (que expresa la orientación del pararrotor con respecto a los ejes inerciales) y la velocidad relativa de los ejes cuerpo respecto de los inerciales.

\subsubsection{Velocidades relativas}

Se considera la velocidad $\mathbf{V}_{t c}$ correspondiente a la velocidad del pararrotor con respecto a los ejes inerciales expresada en coordenadas del cuerpo. Sólo se considera el caso en que $\mathbf{V}_{t c}$ es una velocidad de descenso vertical y unifome, y por tanto tiene la dirección del eje $Z$ inercial (que se puede identificar como eje vertical).

La velocidad del fluido relativa a la pala 1 será: 


$$
\begin{aligned}
& \mathbf{V}_{r 1}=-\left[\begin{array}{l}
\omega_{1} \\
\omega_{2} \\
\omega_{3}
\end{array}\right] \times\left[\begin{array}{l}
r_{11} \\
r_{12} \\
r_{13}
\end{array}\right]+\mathbf{V}_{t c}, \\
& \mathbf{V}_{r 1}=-\left[\begin{array}{c}
\omega_{1} \\
\omega_{2} \\
\omega_{3}
\end{array}\right] \times\left[\begin{array}{c}
r_{11} \\
r_{12} \\
r_{13}
\end{array}\right]+\mathbf{V}_{t c}=\left[\begin{array}{c}
r_{12} \omega_{3}-r_{13} \omega_{2}+U_{\infty} \theta \sin \varphi \\
r_{13} \omega_{1}-r_{11} \omega_{3}+U_{\infty} \theta \cos \varphi \\
r_{11} \omega_{2}-r_{12} \omega_{1}+U_{\infty}
\end{array}\right] .
\end{aligned}
$$

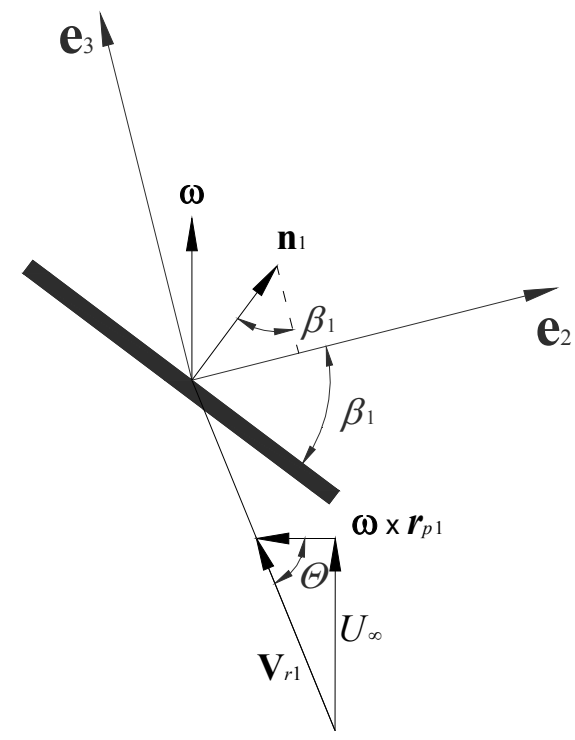

Figura 0.3 Velocidad relativa a la pala 1 

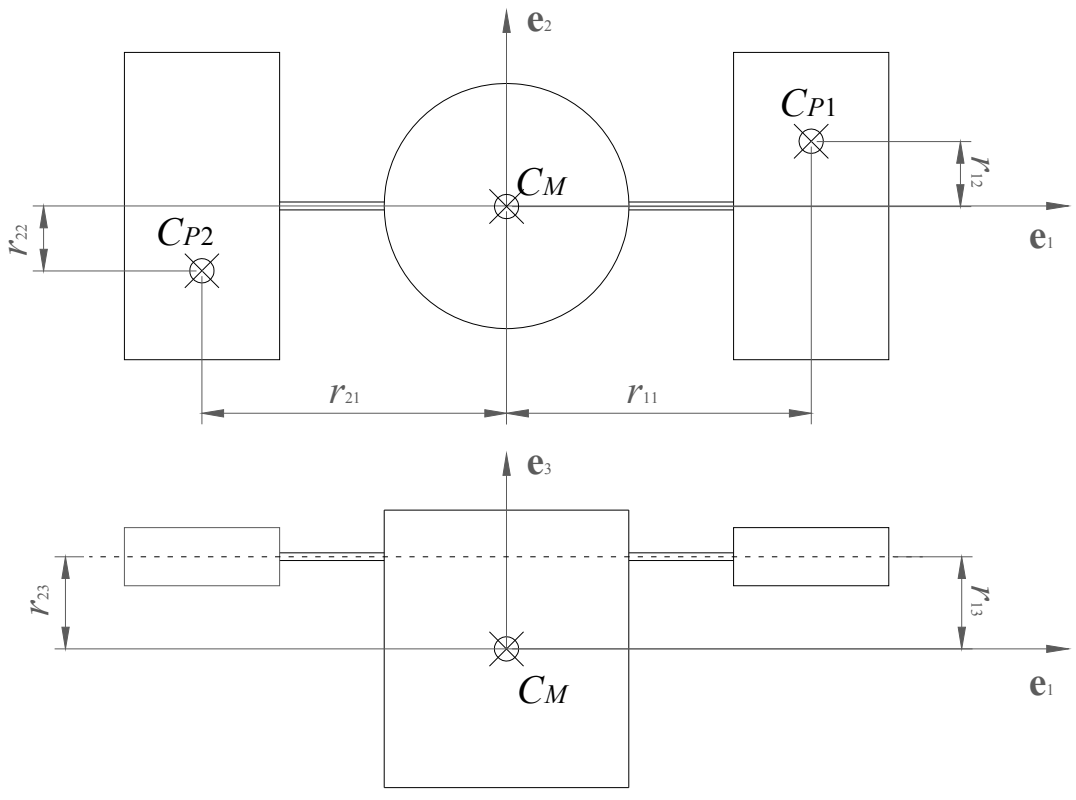

Figura 0.4 Posición del centro de presiones de las palas al respecto del centro de masas.

Considerando que $r_{21}, r_{22}$ y $r_{23}$ son las coordenadas de la posición del centro de presiones de la pala 2 respecto al centro de masas, y que éstas son geométricamente idénticas a las correspondientes a la pala 1 , se tiene, para la pala 2 :

$$
\mathbf{V}_{r 2}=\left[\begin{array}{c}
-r_{22} \omega_{3}-r_{23} \omega_{2}+U_{\infty} \theta \sin \varphi \\
r_{23} \omega_{1}+r_{21} \omega_{3}+U_{\infty} \theta \cos \varphi \\
-r_{21} \omega_{2}+r_{22} \omega_{1}+U_{\infty}
\end{array}\right]
$$

\subsubsection{Velocidades normales}

Considerando los vectores unitarios normales a cada plano de las palas, las componentes de las velocidades normales a las superficies de las palas, $V_{n 1} \mathrm{y} V_{n 2}$, responderán a las siguientes expresiones: 


$$
\begin{gathered}
V_{n 1}=\mathbf{n}_{1} \cdot \mathbf{V}_{r 1}=\left[\begin{array}{c}
0 \\
\sin \beta_{1} \\
\cos \beta_{1}
\end{array}\right] \cdot\left[\begin{array}{c}
r_{12} \omega_{3}-r_{13} \omega_{2}+U_{\infty} \theta \sin \varphi \\
r_{13} \omega_{1}-r_{11} \omega_{3}+U_{\infty} \theta \cos \varphi \\
r_{11} \omega_{2}-r_{12} \omega_{1}+U_{\infty}
\end{array}\right], \\
V_{n 2}=\mathbf{n}_{2} \cdot \mathbf{V}_{r 2}=\left[\begin{array}{c}
0 \\
-\sin \beta_{2} \\
\cos \beta_{2}
\end{array}\right] \cdot\left[\begin{array}{c}
-r_{22} \omega_{3}-r_{23} \omega_{2}+U_{\infty} \theta \sin \varphi \\
r_{23} \omega_{1}+r_{21} \omega_{3}+U_{\infty} \theta \cos \varphi \\
-r_{21} \omega_{2}+r_{22} \omega_{1}+U_{\infty}
\end{array}\right] .
\end{gathered}
$$

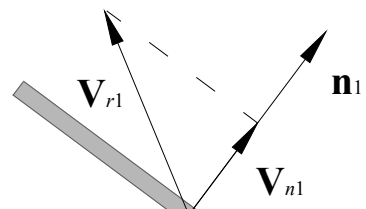

Figura 0.5 Determinación del ángulo de ataque para la pala 1.

Considerando pequeños ángulos de paso de las palas, se tiene:

$$
\begin{aligned}
& V_{n 1}=r_{11} \omega_{3}\left[\frac{\beta_{1} r_{13} \omega_{1}}{r_{11} \omega_{3}}-\beta_{1}+\frac{\beta_{1} U_{\infty} \theta \cos \varphi}{r_{11} \omega_{3}}+\frac{\omega_{2}}{\omega_{3}}-\frac{r_{12} \omega_{1}}{r_{11} \omega_{3}}+\frac{U_{\infty}}{r_{11} \omega_{3}}\right], \\
& V_{n 2}=r_{11} \omega_{3}\left[-\frac{\beta_{2} r_{13} \omega_{1}}{r_{11} \omega_{3}}-\beta_{2}-\frac{\beta_{2} U_{\infty} \theta \cos \varphi}{r_{11} \omega_{3}}-\frac{\omega_{2}}{\omega_{3}}+\frac{r_{12} \omega_{1}}{r_{11} \omega_{3}}+\frac{U_{\infty}}{r_{11} \omega_{3}}\right] .
\end{aligned}
$$

\subsubsection{Versores}

Se consideran una serie de vectores normalizados en las direcciones de la sustentación y de la resistencia asociadas a cada pala, para, a posteriori, asignarles las magnitudes de las fuerzas aerodinámicas. El vector sustentación será normal al plano formado por la velocidad relativa y el eje según la envergadura de la pala que se esté considerando. 
El módulo del vector que resulta del producto vectorial entre un vector unitario según la envergadura de la pala y la velocidad relativa es igual al módulo de la velocidad relativa si esta no tiene componentes según la envergadura (o mejor dicho si ésta es perpendicular a la envergadura).

Vale decir que estos versores serán:

$\mathbf{e}_{L 1}=\frac{-\left[\begin{array}{l}1 \\ 0 \\ 0\end{array}\right] \times \mathbf{V}_{r 1}}{\left[\begin{array}{l}1 \\ 0 \\ 0\end{array}\right] \times \mathbf{V}_{r 1} \mid} \cong\left[\begin{array}{c}0 \\ \frac{r_{11} \omega_{3}\left(\frac{\omega_{2}}{\omega_{3}}-\frac{r_{12}}{r_{11}} \frac{\omega_{1}}{\omega_{3}}+\frac{U_{\infty}}{r_{11} \omega_{3}}\right)}{\left|\mathbf{V}_{r 1}\right|} \\ \frac{r_{11} \omega_{3}\left(1-\frac{r_{13}}{r_{11}} \frac{\omega_{1}}{\omega_{3}}-\frac{U_{\infty} \theta \cos \varphi}{r_{11} \omega_{3}}\right)}{\left|\mathbf{V}_{r 1}\right|}\end{array}\right]$

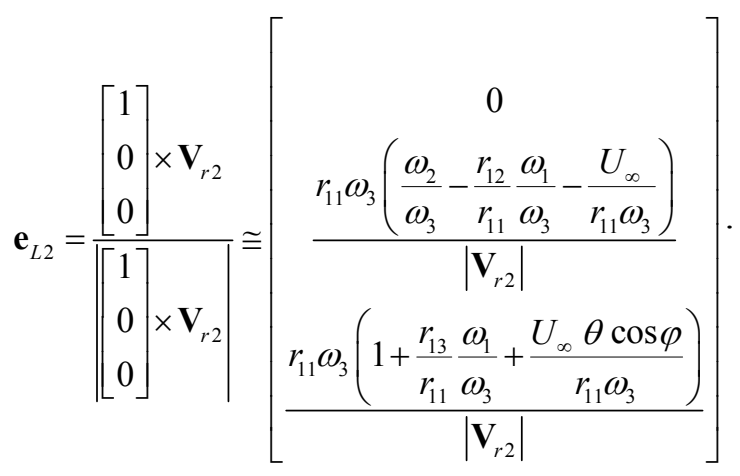




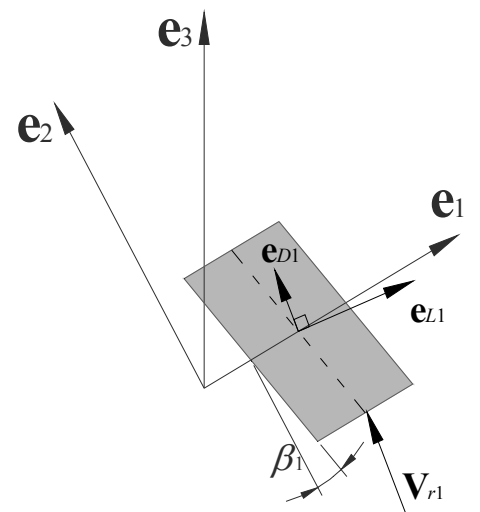

Figura 0.6 Versores asociados a la pala 1

Los versores resistencia tendrán la dirección de la velocidad relativa a cada pala:

$\mathbf{e}_{D i}=\frac{\mathbf{V}_{r i}}{\left|\mathbf{V}_{r i}\right|}$

$\mathbf{e}_{D 1}=\frac{\mathbf{V}_{r 1}}{\left|\mathbf{V}_{r 1}\right|}=\left[\begin{array}{c}\frac{r_{12} \omega_{3}-r_{13} \omega_{2}+U_{\infty} \theta \sin \varphi}{\left|\mathbf{V}_{r 1}\right|} \\ \frac{r_{13} \omega_{1}-r_{11} \omega_{3}+U_{\infty} \theta \cos \varphi}{\left|\mathbf{V}_{r 1}\right|} \\ \frac{r_{11} \omega_{2}-r_{12} \omega_{1}+U_{\infty}}{\left|\mathbf{V}_{r 1}\right|}\end{array}\right]$,

$\mathbf{e}_{D 2}=\frac{\mathbf{V}_{r 2}}{\left|\mathbf{V}_{r 2}\right|}=\left[\begin{array}{c}\frac{-r_{12} \omega_{3}-r_{13} \omega_{2}+U_{\infty} \theta \sin \varphi}{\left|\mathbf{V}_{r 2}\right|} \\ \frac{r_{13} \omega_{1}+r_{11} \omega_{3}+U_{\infty} \theta \cos \varphi}{\left|\mathbf{V}_{r 2}\right|} \\ \frac{-r_{11} \omega_{2}+r_{12} \omega_{1}+U_{\infty}}{\left|\mathbf{V}_{r 2}\right|}\end{array}\right]$. 


\subsection{6 Ángulos de ataque}

Los ángulos de ataque para las palas $i=1,2$ serán:

$$
\sin \alpha_{i}=\frac{\mathbf{n}_{i} \cdot \mathbf{V}_{r i}}{\left|\mathbf{V}_{r i}\right|}
$$

Si los ángulos de ataque son pequeños, puede aproximarse $\sin \alpha_{i}$ por $\alpha_{i}$.

\subsubsection{Fuerzas aerodinámicas}

Las fuerzas de sustentación y resistencia se obtienen reemplazando (11) (12) (14) (15) en:

$$
\begin{aligned}
& \mathbf{L}_{i}=\frac{1}{2} \rho S C_{L \alpha} \alpha_{\mathrm{i}}\left|\mathbf{V}_{r i}\right|^{2} \mathbf{e}_{L i}, \\
& \mathbf{D}_{i}=\frac{1}{2} \rho S C_{D}\left|\mathbf{V}_{r i}\right|^{2} \mathbf{e}_{D i} .
\end{aligned}
$$

\subsubsection{Simplificación de las expresiones de fuerzas}

Para evaluar los órdenes de magnitud de las ecuaciones de momentos, se toma a la unidad como orden “ 0 ”. Entonces, se tiene que:

- $\quad \theta$ es de orden 1.

- $\quad \beta_{1} \mathrm{y} \beta_{2}$ son de orden 1.

- $\quad k=U_{\infty} /\left(\omega_{3} r_{11}\right)$ es de orden 1.

- $\quad k_{21}$ es de orden 1.

- $\quad C_{D}$ es de orden 1.

- los parámetros geométricos y aerodinámicos de ambas palas son idénticos, salvo su ángulo de paso. 
- $\quad$ las fuerzas aerodinámicas no poseen componente en dirección de la envergadura de las palas.

Se desarrollan las expresiones de los momentos y se retienen términos hasta de orden 2, a partir de las ecuaciones (17) y (18):

$$
L_{11}=0
$$

$L_{12}=\frac{1}{2} \rho S C_{L \alpha} r_{11}{ }^{2} \omega_{3}^{2}\left(-\beta_{1}+x_{2}+k\right)\left(x_{2}+k\right)$,

$L_{12}=\frac{1}{2} \rho S C_{L \alpha} r_{11}{ }^{2} \omega_{3}{ }^{2}\left(-\beta_{1} x_{2}-\beta_{1} k+x_{2}{ }^{2}+2 x_{2} k+k^{2}\right)$,

$L_{13}=\frac{1}{2} \rho S C_{L \alpha}{r_{11}}^{2} \omega_{3}^{2}\left(-\beta_{1}+x_{2}+k\right)$,

$L_{21}=0$,

$L_{22}=\frac{1}{2} \rho S C_{L \alpha} r_{11}^{2} \omega_{3}^{2}\left(-\beta_{2}-x_{2}+k\right)\left(x_{2}-k\right)$,

$L_{22}=\frac{1}{2} \rho S C_{L \alpha} r_{11}{ }^{2} \omega_{3}{ }^{2}\left(-\beta_{2} x_{2}+\beta_{2} k-x_{2}{ }^{2}+2 x_{2} k-k^{2}\right)$,

$L_{23}=\frac{1}{2} \rho S C_{L \alpha} r_{11}^{2} \omega_{3}^{2}\left(-\beta_{2}-x_{2}+k\right)$,

$D_{11}=0$,

$D_{12}=\frac{1}{2} \rho S C_{D} r_{11}^{2} \omega_{3}^{2}\left(x_{1} k_{31}-1\right)$,

$D_{13}=\frac{1}{2} \rho S C_{D} r_{11}^{2} \omega_{3}^{2}\left(x_{2}-k_{21}+k\right)$, 
$D_{21}=0$,

$D_{22}=\frac{1}{2} \rho S C_{D} r_{11}^{2} \omega_{3}^{2}\left(x_{1} k_{31}+1\right)$,

$D_{23}=\frac{1}{2} \rho S C_{D} r_{11}^{2} \omega_{3}^{2}\left(-x_{2}+k_{21}+k\right)$.

\subsubsection{Momentos respecto al centro de masas}

El momento generado por las fuerzas aerodinámicas de ambas palas expresados, en función de las componentes de cada vector, está dado por:

$\mathbf{M}=\left[\left[\begin{array}{l}r_{11} \\ r_{12} \\ r_{13}\end{array}\right] \times\left[\begin{array}{l}L_{11} \\ L_{12} \\ L_{13}\end{array}\right]+\left[\begin{array}{c}r_{11} \\ r_{12} \\ r_{13}\end{array}\right] \times\left[\begin{array}{c}D_{11} \\ D_{12} \\ D_{13}\end{array}\right]+\left[\begin{array}{c}-r_{11} \\ -r_{12} \\ r_{13}\end{array}\right] \times\left[\begin{array}{c}L_{21} \\ L_{22} \\ L_{23}\end{array}\right]+\left[\begin{array}{c}-r_{11} \\ -r_{12} \\ r_{13}\end{array}\right] \times\left[\begin{array}{c}D_{11} \\ D_{12} \\ D_{23}\end{array}\right]\right]$,

donde $k_{21}=r_{12} / r_{11}$ y $k_{31}=r_{13} / r_{11}$

$\mathbf{M}=r_{11}\left[\begin{array}{c}-\left(L_{12} k_{31}-L_{13} k_{21}+D_{12} k_{31}-D_{13} k_{21}\right)+\left(-L_{22} k_{31}-L_{23} k_{21}-D_{22} k_{31}-D_{23} k_{21}\right) \\ -\left(L_{13}+D_{13}-D_{11} k_{31}\right)+\left(L_{23}+D_{23}+D_{21} k_{31}\right) \\ -\left(-L_{12}+D_{11} k_{21}-D_{12}\right)-\left(L_{22}-D_{21} k_{21}-D_{22}\right)\end{array}\right]$,

donde $k_{21}=r_{12} / r_{11}$ y $k_{31}=r_{13} / r_{11}$.

\subsubsection{Simplificación de las expresiones de momentos}

Teniendo en cuenta las consideraciones en 2.2.7.1 en cuanto a órdenes de magnitud, se desarrollan las expresiones de los momentos y se retienen términos hasta de orden 2, a partir de la ecuación (32). Se define el coeficiente de momentos, $C_{m i}$, con la ayuda de la expresión que sigue:

$\frac{M_{1}}{I_{1}}=\frac{1}{2} \frac{\rho S r_{11}{ }^{3}}{I_{1}} \omega_{0}^{2} C_{m 1}=K_{1} \omega_{0}^{2} C_{m 1}$, 
donde $K_{i}=\frac{1}{2} \frac{\rho S r_{11}{ }^{3}}{I_{i}}$ es adimensional.

$C_{m i}$ para los ejes $i=1,2,3$, responde a:

$$
\begin{aligned}
C_{m 1} & =C_{L \alpha} k_{31}\left[\left(\beta_{1}+\beta_{2}\right) \frac{\omega_{2}}{\omega_{3}}+\left(\beta_{1}-\beta_{2}\right) k-4 \frac{\omega_{2}}{\omega_{3}} k\right]+ \\
& +C_{L \alpha} k_{21}\left[-\beta_{1}+\beta_{2}+2 \frac{\omega_{2}}{\omega_{3}}\right]+C_{D} k_{31}\left[-4 \frac{\omega_{1}}{\omega_{3}} k_{31}\right] \\
C_{m 2} & =-C_{L \alpha}\left[-\beta_{1}+\beta_{2}+2 \frac{\omega_{2}}{\omega_{3}}\right]-2 C_{D} \frac{\omega_{2}}{\omega_{3}} \\
C_{m 3} & =C_{L \alpha}\left[-\left(\beta_{1}-\beta_{2}\right) \frac{\omega_{2}}{\omega_{3}}-\left(\beta_{1}+\beta_{2}\right) k+2\left(\frac{\omega_{2}}{\omega_{3}}\right)^{2}+2 \Theta^{2}\right]+2 C_{D}\left[\frac{\omega_{1}}{\omega_{3}} k_{31}\right] .
\end{aligned}
$$

Si $r_{13}=0$ (el eje que contiene a los centros de presiones pasa por el centro de masas), y considerando sólo el orden mayor de cada una de las ecuaciones, se tienen las ecuaciones halladas por Nadal Mora (2005).

\subsubsection{Linealización del sistema de ecuaciones}

Para la linealización del sistema y su posterior análisis se adopta como punto de partida las ecuaciones de la dinámica del pararrotor (ecuaciones (1) a (3)). Se considera, de acuerdo con lo desarrollado por Nadal Mora (2005), que $\omega_{3}$ es constante en el entorno de una solución de equilibrio $\left(\omega_{3}=\omega_{0}\right)$, en consecuencia, las ecuaciones (1) y (2) quedan desacopladas de la (3). Es posible por tanto realizar un cambio de variables a las nuevas $x_{1}=\omega_{1} / \omega_{0}$ y $x_{2}=\omega_{2} / \omega_{0}$.

Se considera por tanto:

$T=\omega_{0} t \longrightarrow \frac{\mathrm{d} x}{\mathrm{~d} t}=\omega_{0} \frac{\mathrm{d} x}{\mathrm{~d} T}$

El sistema de ecuaciones de la dinámica del pararrotor, entonces, queda: 
$\frac{M_{1}}{I_{1} \omega_{0}^{2}}=\frac{\mathrm{d} x_{1}}{\mathrm{~d} T}-\left(\frac{I_{2}-I_{3}}{I_{1}}\right) x_{2}$

$\frac{M_{2}}{I_{2} \omega_{0}^{2}}=\frac{\mathrm{d} x_{2}}{\mathrm{~d} T}+\left(\frac{I_{1}-I_{3}}{I_{2}}\right) x_{1}$

Reemplazando en las ecuaciones anteriores los coeficientes de momentos (34) y (35), se observa que el sistema así obtenido, que reúne las simplificaciones asumidas para la expresión de los momentos y el desacoplamiento de las rotaciones sobre el eje 3, presenta una no linealidad asociada al parámetro $k_{31}$ (que representa la posición en el eje 3 de las palas respecto al centro de masa), siempre que éste sea suficientemente grande.

El hecho presentado anteriormente puede observarse al expresarse el sistema en la forma:

$\frac{d x_{1}}{d T}=A_{11} x_{1}+A_{12} x_{2}+B_{1}=f_{1}\left(x_{1}, x_{2}\right)$,

donde:

$f_{1}\left(x_{1}, x_{2}\right)=\frac{I_{2}-I_{3}}{I_{1}} x_{2}$
$+\frac{K}{\omega_{0}{ }^{2}}\left\{C_{L \alpha} k_{31}\left[\left(\beta_{1}+\beta_{2}\right) x_{2}+\left(\beta_{1}-\beta_{2}\right) \Theta-4 x_{2} \Theta\right]+C_{L \alpha} k_{21}\left(-\beta_{1}+\beta_{2}+2 x_{2}\right)-4 C_{D} k_{31}{ }^{2} x_{1}\right\}$

y también:

$\frac{\mathrm{d} x_{2}}{\mathrm{~d} T}=A_{21} x_{1}+A_{22} x_{2}+B_{2}=f_{2}\left(x_{1}, x_{2}\right)$,

donde:

$f_{2}\left(x_{1}, x_{2}\right)=\frac{K}{\omega_{0}^{2}}\left(-C_{L \alpha}\left[-\beta_{1}+\beta_{2}+2 x_{2}\right]-2 C_{D} x_{2}\right)-\frac{I_{1}-I_{3}}{I_{2}} x_{1}$.

Las expresiones (33) y (35) muestran los coeficientes $A_{i j}$ y $B_{i j}$ del sistema de ecuaciones. 
Una metodología posible para analizar la estabilidad del sistema de ecuaciones en las cercanías de un punto de equilibrio resulta de linealizar las Ecuaciones (39) y (41), según:

$$
\frac{\mathrm{d} x_{i}}{\mathrm{~d} t}=f_{i}\left(x_{e q 1}, x_{e q 2}\right)+\frac{\partial f_{i}\left(x_{e q 1}, x_{e q 2}\right)}{\partial x_{1}}\left(x_{1}-x_{e q 1}\right)+\frac{\partial f_{i}\left(x_{e q 1}, x_{e q 2}\right)}{\partial x_{2}}\left(x_{2}-x_{e q 2}\right),
$$

donde $i=1,2$.

Para analizar la estabilidad del sistema en un entorno del punto de equilibrio se evalúan los autovalores de la matriz $\mathbf{A}$, conformada por:

$$
\mathbf{A}=\left[\begin{array}{ll}
A_{11} & A_{12} \\
A_{21} & A_{22}
\end{array}\right] \quad,
$$

para un sistema con dos grados de libertad, donde $A_{i j}=\partial f_{i} / \partial x_{j}$, particularizada en $x_{i}=x_{\text {eqi }}$. Estos elementos son aquellos mencionados en las ecuaciones (39) y (41).

Los autovalores de la matriz A tendrán la forma:

$$
\lambda_{1,2}=\frac{\left(A_{11}+A_{22}\right) \pm \sqrt{\left(A_{11}+A_{22}\right)^{2}+4\left(A_{12} A_{21}\right)}}{2}
$$

El sistema lineal será marginalmente estable sí y sólo sí la parte real de los autovalores de $A$ son menores o iguales a cero. En el caso que exista una raíz con parte real igual a 0 deberá cumplir la condición de que estas raíces sean simples del polinomio característico de $A$ (autovalor de multiplicidad 1). El sistema lineal será asintóticamente estable sí y sólo sí los autovalores de $A$ son menores que cero (la parte real), según Chi-Tson Chen (1999), la cual representa la situación de interés para este análisis. Si $k_{31}$ es de orden 0 y se contemplan los órdenes de magnitud mayores, la matriz $A$ está dada por: 


$$
\mathbf{A}=\left[\begin{array}{cc}
\frac{1}{2} \frac{\rho S r_{11}{ }^{3}}{I_{1}}\left(-4 C_{D} k_{31}{ }^{2}\right) & \frac{I_{2}-I_{3}}{I_{1}}+\frac{1}{2} \frac{\rho S r_{11}{ }^{3}}{I_{1}}\left(C_{L \alpha} k_{31}\left(\beta_{1}+\beta_{2}-4 k\right)+2 C_{L \alpha} k_{21}\right) \\
-\frac{I_{1}-I_{3}}{I_{2}} & \frac{1}{2} \frac{\rho S r_{11}{ }^{3}}{I_{2}} 2\left(-C_{L \alpha}-C_{D}\right)
\end{array}\right],
$$

mientras que si $k_{31}$ es de orden 1 , haciendo idéntica consideración al respecto de los órdenes de magnitud, la expresión es:

$$
\mathbf{A}=\left[\begin{array}{cc}
0 & \frac{I_{2}-I_{3}}{I_{1}}+\frac{1}{2} \frac{\rho S r_{11}^{3}}{I_{1}}\left(2 C_{L \alpha} k_{21}\right) \\
-\frac{I_{1}-I_{3}}{I_{2}} & \frac{1}{2} \frac{\rho S r_{11}^{3}}{I_{2}} 2\left(-C_{L \alpha}-C_{D}\right)
\end{array}\right]
$$

En posible observar que en la expresión anterior no aparece el término $k_{31}$, y la matriz $\mathbf{A}$ es la obtenida para el caso en que la posición de los centros de presiones está en la misma cota que el centro de masas del pararrotor, congruente con lo desarrollado por Nadal Mora (2005).

\subsection{ANÁLISIS DEL EQUILIBRIO}

La solución de equilibrio en la velocidad de rotación del modelo de la dinámica rotacional del pararrotor se obtiene adoptando la condición de aceleración nula de las velocidades angulares, resultando el siguiente sistema de ecuaciones:

$$
\begin{aligned}
& K_{1} C_{m 1}=-\left(\frac{I_{2}-I_{3}}{I_{1}}\right) x_{e q 2}, \\
& K_{2} C_{m 2}=\left(\frac{I_{1}-I_{3}}{I_{2}}\right) x_{e q 1},
\end{aligned}
$$

donde: 
$K_{1}=\frac{1}{2} \frac{\rho S r_{11}{ }^{3}}{I_{1}}$,

$K_{2}=\frac{1}{2} \frac{\rho S r_{11}^{3}}{I_{2}}$.

Se asumirá que las constantes que aquí se denominan $K_{i}, i=1,2$, constantes de relación de inercia, son de orden 2 en la generalidad de los casos. Se realiza esta consideración a partir de la interrelación que existe entre parámetros geométricos que definen su orden de magnitud.

En este sentido es de destacar en particular que la relación $\rho / \rho_{m}$, donde $\rho_{m}=\frac{I_{1}}{S r_{11}{ }^{3}}$, es un factor determinante del orden de magnitud de $K_{i}$.

Empleando esta notación y las ecuaciones (39) y (41) se obtiene:

$$
\begin{aligned}
& \frac{I_{3}-I_{2}}{I_{1}} x_{e q 2}= \\
& K_{1}\left\{C_{L \alpha} k_{31}\left[\left(\beta_{1}+\beta_{2}\right) x_{e q 2}+\left(\beta_{1}-\beta_{2}\right) k-4 x_{e q 2} k\right]\right\}+K_{1} C_{L \alpha} k_{21}\left[-\beta_{1}+\beta_{2}+2 x_{e q 2}\right]-K_{1}\left(4 C_{D} x_{e q 1} k_{31}{ }^{2}\right), \\
& \left(\frac{I_{1}-I_{3}}{I_{2}}\right) x_{e q 1}=K_{2}\left(-C_{L \alpha}\left[-\beta_{1}+\beta_{2}+2 x_{e q 2}\right]-2 C_{D} x_{e q 2}\right) .
\end{aligned}
$$

Entonces, si consideramos que el último término de la ecuación (52) es de un orden menor al resto de los términos por ser $C_{D}$ mucho menor que $C_{L \alpha}$ resulta:

$$
\begin{aligned}
& x_{e q 2}=\frac{2 K_{1} C_{L \alpha} \delta_{\beta}\left(-k_{31} k+k_{21}\right)}{2 K_{1} C_{L \alpha}\left(k_{31} \beta_{0}-2 k_{31} k+k_{21}\right)+\frac{I_{2}-I_{3}}{I_{1}}} \\
& x_{e q 1}=\frac{2 K_{2}\left(C_{L \alpha} \delta_{\beta}-\left(C_{D}+C_{L \alpha}\right) x_{e q 2}\right)}{\frac{I_{1}-I_{3}}{I_{2}}}
\end{aligned}
$$


Las expresiones que surgen del modelo desarrollado por Nadal Mora (2005) respecto del punto de equilibrio resultan congruentes con las anteriores, para $k_{31}=0$, considerando los órdenes de los componentes presentes. Estas son:

$$
\begin{aligned}
& x_{e q 2}=\frac{2 \delta_{\beta} C_{L \alpha} k_{21} K_{1}}{2 C_{L \alpha} k_{21} K_{1}+\left(\frac{I_{2}-I_{3}}{I_{1}}\right)}, \\
& x_{e q 1}=\frac{-2 K_{2} C_{L \alpha} x_{e q 2}+2 K_{2} C_{L \alpha} \delta_{\beta}}{\left(\frac{I_{1}-I_{3}}{I_{2}}\right)} .
\end{aligned}
$$

\subsubsection{Variable $x_{e q 2}$}

Al analizar los órdenes de magnitud del estado de equilibrio (54) de la variable $x_{e q 2}$, se encuentra que:

$x_{e q 2}=\frac{-O(4)+O(4)}{O(3)-O(3)+O(3) \pm O(1)}$.

En este análisis de órdenes de magnitud se consideraron $k_{31}$ y $\delta_{\beta}$ como positivos. En adelante consideraremos que $\delta_{\beta}$ siempre toma un valor positivo o nulo como acuerdo de disposición de los ángulos de ataque de las palas.

Ambos términos del numerador de la expresión anterior son dependientes de $\delta_{\beta}$. Si este parámetro es nulo, entonces la variable $x_{e q 2}$ en el equilibrio será 0 . Si $\delta_{\beta}$ no es nulo entonces el efecto de los parámetros $k_{31}$ y $k_{21}$ será determinante en la solución de $x_{\text {eq2 }}$.

Se puede observar que en el denominador el término de orden 1 que incluye la diferencia entre $I_{2}$ e $I_{3}$ es dominante.

Con la intención de analizar el signo y orden de magnitud de $x_{e q 2}$ podemos ver que: 
- $\quad$ El orden de magnitud de $x_{e q 2}$ es 3

- El signo que tomará $x_{e q 2}$ depende principalmente de los parámetros $\delta_{\beta}, k_{31}, I_{2}-I_{3}$

- $\quad$ Si $\delta_{\beta}=0$, entonces $x_{e q 2}=0$, lo que significa que $\omega_{e q 2}=0$.

Los casos que se presentan se resumen en la Tabla 2.1.

Tabla 0.1 Signos que toma $x_{e q 2}$ frente a la variación de parámetros determinantes

\begin{tabular}{|c|c|c|}
\hline $\begin{array}{c}\text { Condición } \\
\text { para } \boldsymbol{k}_{\mathbf{3 1}}\end{array}$ & $\begin{array}{c}\text { Condición } \\
\text { para } \boldsymbol{I}_{\mathbf{3}}, \boldsymbol{I}_{\mathbf{2}}\end{array}$ & $\boldsymbol{x}_{\boldsymbol{e q 2}}$ \\
\hline \multirow{2}{*}{$>0$} & $I_{3}>I_{2}$ & $>0$ si $k_{31} k>k_{21}$ \\
\cline { 2 - 3 } & $I_{3}<I_{2}$ & $<0$ si $k_{31} k>k_{21}$ \\
\hline \multirow{2}{*}{$=0$} & $I_{3}>I_{2}$ & $<0$ \\
\cline { 2 - 3 } & $I_{3}<I_{2}$ & $>0$ \\
\hline \multirow{2}{*}{$<0$} & $I_{3}>I_{2}$ & $<0$ \\
\cline { 2 - 3 } & $I_{3}<I_{2}$ & $>0$ \\
\hline
\end{tabular}

Del análisis de los casos se deduce que existe una determinada magnitud positiva de $k_{31}$ a partir de la cual $x_{e q 2}>0$ para $I_{3}>I_{2}$, o $x_{e q 2}<0$ para $I_{3}<I_{2}$.

\subsubsection{Variable $x_{e q 1}$}

Al analizar los órdenes de magnitud la posición de equilibrio (55) de la variable $x_{e q 1}$, se encuentra que:

$x_{e q 1}=\frac{2 K_{2}\left(-x_{e q 2}\left(C_{D}+C_{L \alpha}\right)+C_{L \alpha} \delta_{\beta}\right)}{\left(\frac{I_{1}-I_{3}}{I_{2}}\right)}$,

que en forma de órdenes de magnitud se puede expresar como: 
$x_{e q 1}=\frac{-O(4)-O(3)+O(3)}{ \pm O(1)}$.

Las expresiones de orden 3 del numerador preponderan, y por tanto estas definen si es mayor o menor que 0 (salvo que se anulen). Si se calcula la suma de estas expresiones se obtiene que:

numerador $\left(x_{e q 1}\right) \approx 2 K_{2} C_{L \alpha}\left(\delta_{\beta}-x_{e q 2}\right) \approx 2 K_{2} C_{L \alpha} \frac{\delta_{\beta} \frac{I_{2}-I_{3}}{I_{1}}}{2 K_{1} C_{L \alpha}\left(k_{31} \beta_{0}-2 k_{31} k+k_{21}\right)+\frac{I_{2}-I_{3}}{I_{1}}}$.

Por lo tanto se puede ver que el signo del numerador dependerá principalmente de $I_{2}-I_{3}$ y de $\delta_{\beta}$. Este hecho implica que tanto en el numerador como el denominador de la expresión (60) prepondera la diferencia $I_{2}-I_{3}$ (si esta es lo suficientemente grande), lo cual implica que (60) será positivo si $\delta_{\beta}$ es positivo (siempre que $I_{2}-I_{3}$ sea suficientemente grande).

Se puede observar que en el denominador de (58) el término de orden 1 que incluye la diferencia entre $I_{1}$ e $I_{3}$ resulta dominante.

Con la intención de analizar el signo y orden de magnitud del equilibrio de la variable podemos ver que:

- $\quad$ El orden de magnitud de $x_{e q 1}$ es 2.

- $\quad$ El signo que tomará $x_{e q 2}$ depende principalmente de $I_{2}-I_{3}, I_{1}-I_{3}, k_{31}$.

- $\quad$ Si $\delta_{\beta}=0$, la solución es $x_{e q 1}=0$ y $x_{e q 2}=0$, lo cual implica que $\omega_{e q 1}=0$ y $\omega_{e q 2}=0$.

Los casos que se presentan se resumen en la Tabla 2.2. 
Tabla 0.2 Signos que toma $x_{e q 1}$ frente a la variación de parámetros determinantes, para valores de $\delta_{\beta}>0$.

\begin{tabular}{|c|c|c|}
\hline $\begin{array}{c}\text { Condición } \\
\text { para } \boldsymbol{k}_{\mathbf{3 1}}\end{array}$ & $\begin{array}{c}\text { Condición } \\
\text { para } \boldsymbol{I}_{\mathbf{3}}, I_{\mathbf{1}}\end{array}$ & $\boldsymbol{x}_{\boldsymbol{e q} \mathbf{1}}$ \\
\hline \multirow{2}{*}{$>0 ;<0$} & $I_{3}>I_{1}$ & $<0$ \\
\cline { 2 - 3 } & $I_{3}<I_{1}$ & $>0$ \\
\hline
\end{tabular}

\subsubsection{Conclusiones}

De lo expuesto en los párrafos anteriores se deduce que el orden de magnitud de las variables en el equilibrio es al menos 2 , y que sus signos dependen de los momentos de inercia y del parámetro $k_{31}$.

En la Tabla 2.3 se resumen los signos del valor que toman las variables en el equilibrio con relación a los momentos de inercia y $k_{31}$.

Tabla 0.3 Signos de las variables en equilibrio para variantes de sus parámetros determinantes, para valores de $\delta_{\beta}>0$.

\begin{tabular}{|c|c|c|c|}
\hline $\begin{array}{c}\text { Condición de } \\
\text { momentos de inercia }\end{array}$ & $\begin{array}{c}\text { Condición } \\
\text { para } \boldsymbol{k}_{\mathbf{3 1}}\end{array}$ & $\boldsymbol{x}_{\boldsymbol{e q} \mathbf{1}}$ & $\boldsymbol{x}_{\boldsymbol{e q} \mathbf{2}}$ \\
\hline \multirow{3}{*}{$I_{3}>I_{2}, I_{1}$} & $>0$ & $<0$ & $>0$ si $k_{31} k>k_{21}$ \\
\cline { 2 - 4 } & $=0$ & $<0$ & $<0$ \\
\cline { 2 - 4 } & $<0$ & $<0$ & $<0$ \\
\hline \multirow{3}{*}{$I_{3}<I_{2}, I_{1}$} & $>0$ & $>0$ & $<0$ si $k_{31} k<k_{21}$ \\
\cline { 2 - 4 } & $=0$ & $>0$ & $>0$ \\
\cline { 2 - 4 } & $>0$ & $>0$ & $>0$ \\
\hline
\end{tabular}

\subsection{CRITERIOS DE ESTABILIDAD}

El estudio de la estabilidad está basado en el análisis de la ecuación característica del sistema: 
$\lambda^{2}-\operatorname{Tr} \lambda+D e t=0$

donde las raíces son $\lambda_{1,2}=\frac{1}{2}[\operatorname{Tr} \pm \sqrt{\Delta}]$, siendo el discriminante $\Delta=\operatorname{Tr}^{2}-4$ Det

Del análisis de los signos del discriminante, traza y determinante se obtienen los diferentes tipos de trayectorias que pueden producirse, que se resumen en la Fig. 2.7 (Hirsch \& Smale, 1983).

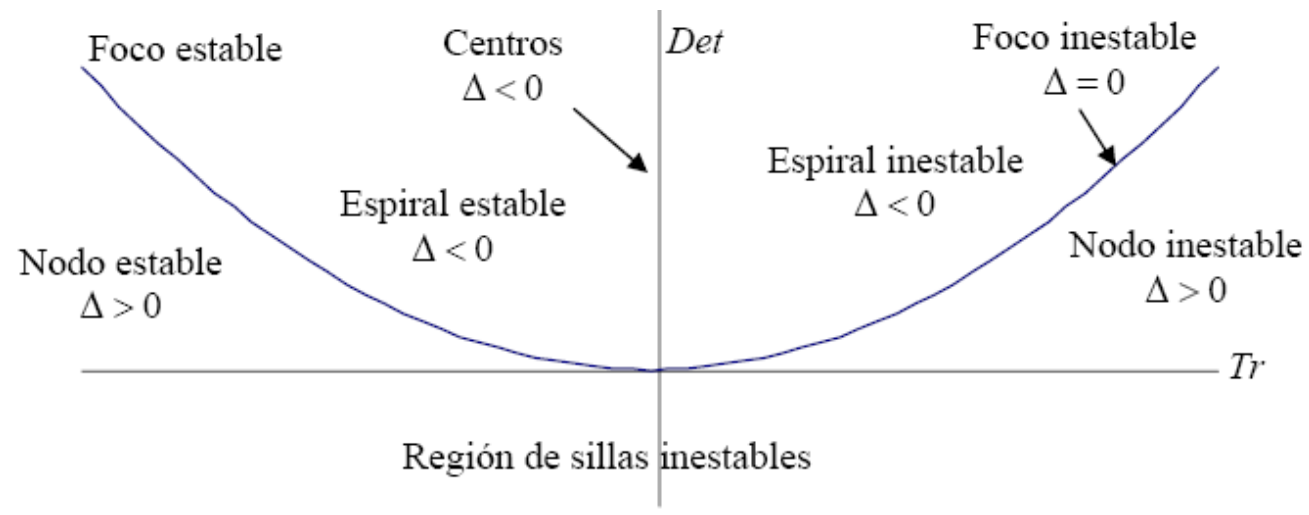

Figura 0.7 Tipos de trayectorias en función de los valores de la traza $T r$, del determinante Det, y del discriminante $\Delta$, de la matriz característica $\mathbf{A}$.

El análisis de la estabilidad de una matriz como la presentada en (44), proveniente de la linealización del sistema de ecuaciones de la dinámica del cuerpo rígido, supone establecer tres casos: cuando las raíces son imaginarias, cuando son reales y coincidentes, y cuando son reales y distintas. Para estos tres casos, la estabilidad asintótica del sistema linealizado se dará cuando la parte real de las raíces de la matriz característica sea menor que cero.

Para que el sistema sea estable debe ocurrir que (Elsgoltz, 1977):

- $\quad$ Si las raíces son reales y diferentes, debe ser $\lambda_{1}<0$ y $\lambda_{2}<0$ (nodo estable). Por tanto $T r$ $<0$, Det $>0, \Delta>0$. 
- $\quad$ Si las raíces son complejas, debe ser $\lambda_{1,2}=p \pm q i, p<0, q \neq 0$ (espiral estable). Por tanto $\operatorname{Tr}<0$, Det $>0, \Delta<0$.

- $\quad$ Si las raíces son iguales, debe ser $\lambda_{1}=\lambda_{2}<0$ (foco estable). Por tanto $\operatorname{Tr}<0$,

$$
\text { Det }=0 \text {. }
$$

Los límites de estabilidad son entonces $\operatorname{Tr}=0$ y $D e t=0$.

\subsubsection{Nodo estable}

Para que las dos raíces del sistema dado por (37) y (38), sean reales y negativas se debe cumplir el criterio de Routh-Hurwitz (Guzmán, 1987), en este caso, siempre que $\Delta>0$ :

$$
\begin{aligned}
& \text { i) } \operatorname{Tr}=A_{11}+A_{22}<0, \\
& \text { ii) } \operatorname{Det}=-A_{12} A_{21}>0 \text {. }
\end{aligned}
$$

La condición $i$ ) se puede expresar como:

$$
\operatorname{Tr}=\frac{1}{2} \rho S r_{11}{ }^{3}\left(-\frac{2}{I_{2}}\left(C_{L \alpha}+C_{D}\right)-\frac{4}{I_{1}} C_{D} k_{31}^{2}\right)<0,
$$

o bien:

$$
\operatorname{Tr}=\rho S r_{11}^{3}\left(\frac{C_{L \alpha}+C_{D}}{I_{2}}+\frac{2 C_{D} k_{31}^{2}}{I_{1}}\right)>0
$$

Es posible observar que la condición dada por (64) se cumple en cualquier caso. Por otro lado el parámetro $k_{31}$ refuerza el cumplimiento de esta condición independientemente de su signo. El orden del término que contiene al parámetro $k_{31}$ es el que prepondera.

La condición ii) se puede expresar como: 
$-\operatorname{Det}=\left(\frac{I_{2}-I_{3}}{I_{1}}+K_{1}\left(C_{L \alpha} k_{31}\left(\left(\beta_{1}+\beta_{2}\right)-4 k\right)+2 C_{L \alpha} k_{21}\right)\right)\left(-\frac{I_{1}-I_{3}}{I_{2}}\right)<0$,

o bien,

$-D e t=\frac{\left(I_{3}-I_{2}\right)\left(I_{3}-I_{1}\right)}{I_{2} I_{1}}+-K_{1}\left(C_{L \alpha} k_{31}\left(\beta_{1}+\beta_{2}-4 k\right)+2 C_{L \alpha} k_{21}\right)\left(-\frac{I_{1}-I_{3}}{I_{2}}\right)>0$.

Esta ecuación es asimilable a la que se plantea en estudios anteriores realizados por Nadal Mora (2005) cuando se consideran órdenes superiores y $k_{31}=0$.

Si reagrupamos expresiones y realizamos operaciones algebraicas, podemos expresar:

$\left(I_{3}-I_{2}\right)\left(I_{3}-I_{1}\right)-I_{a} C_{L \alpha}\left(k_{31}\left(\beta_{\mathrm{o}}-2 k\right)+k_{21}\right)\left(I_{3}-I_{1}\right)>0$,

donde:

$I_{a}=\rho S r_{11}^{3}$

Entonces podemos expresar la anterior ecuación como:

$\left(I_{3}-I_{2}\right)\left(I_{3}-I_{1}\right)\left(1-N_{e}{ }^{\prime}\right)>0$

donde:

$N_{e}{ }^{\prime}=I_{a} C_{L \alpha} \frac{k_{31}\left(\beta_{0}-2 k\right)+k_{21}}{I_{3}-I_{2}}$,

que se denomina número de estabilidad.

Este parámetro juega un papel semejante a $N_{e}$ definido por Nadal Mora (2005):

$N_{e}=\frac{I_{a} C_{L \alpha}}{I_{3}-I_{2}} k_{21}$

que reprenta el caso para el cual $k_{31}=0$. 
Para el análisis de la estabilidad a partir del cumplimiento de la condición ii), consideraremos el aporte término a término del número de estabilidad $N_{e}$. De acuerdo con (68), los casos en que $I_{3}>I_{2}, I_{1}$ o $I_{3}<I_{2}, I_{1}$, valores de $N_{e}{ }^{\prime}<0$ contribuirán a la estabilidad del sistema y a la inversa para valores de $N_{e}{ }^{\prime}>0$.

Denominamos los términos de $N_{e}$ ' de la siguiente manera:

$$
\begin{aligned}
& N_{e}{ }^{\prime}(i)=I_{a} C_{L \alpha} \frac{k_{31}\left(\beta_{\mathrm{o}}-2 k\right)}{\left(I_{3}-I_{2}\right)}, \\
& N_{e}^{\prime}{ }^{\prime}(i i) \\
& =N_{e}=I_{a} C_{L \alpha} \frac{k_{21}}{\left(I_{3}-I_{2}\right)} .
\end{aligned}
$$

Si analizamos término a término tenemos que:

$$
N_{e(i)}^{\prime}=I_{a} C_{L \alpha} \frac{k_{31}\left(\beta_{\mathrm{o}}-2 k\right)}{I_{3}-I_{2}}=I_{a} C_{L \alpha} \frac{k_{31}\left(\beta_{\mathrm{o}}-2\left(\alpha-\beta_{\mathrm{o}}\right)\right)}{I_{3}-I_{2}}=I_{a} C_{L \alpha} \frac{k_{31}\left(-2 \alpha+3 \beta_{\mathrm{o}}\right)}{I_{3}-I_{2}} .
$$

Como por definición geométrica $\alpha>\beta_{0}$, el numerador de este término será negativo para valores de $k_{31}$ positivos. Por lo tanto, para $k_{31}>0$ surgen dos casos (que resultan de signo opuesto para $k_{31}<0$ ):

si $I_{3}>I_{2}$, entonces $N_{e}{ }^{\prime}(i)<0$,

si $I_{3}<I_{2}$, entonces $N_{e}{ }^{\prime}(i)>0$.

Se puede observar que el numerador del segundo término, $N_{e}{ }^{\prime}(i i)$, resulta siempre positivo considerando que el parámetro $k_{21}>0$. Por lo tanto es posible apreciar la existencia de dos casos:

si $I_{3}>I_{2}$, entonces $N_{e}{ }^{\prime}(i i)>0$,

si $I_{3}<I_{2}$, entonces $N_{e}{ }^{\prime}(i i)<0$. 
En general se puede observar que si $I_{3}>I_{2}$, entonces valores de $k_{31}>0$ contribuyen a la estabilidad del sistema (y a la inversa si $k_{31}<0$ ). Si $I_{3}<I_{2}$ valores de $k_{31}<0$ contribuyen a la estabilidad del sistema (y a la inversa si $k_{31}>0$ ).

La configuración geométrica del pararrotor hace que en general se pueda decir que $I_{2}>I_{1}$, por cuanto las palas del pararrotor se asumieron dispuestas sobre el eje 1. En el caso que $I_{2}<$ $I_{3}<I_{1}$, deberá ser $N_{e}{ }^{\prime}>1$ para que el sistema sea estable.

\subsubsection{Espiral estable}

Este caso ocurre cuando ambas raíces son complejas con parte real negativa, y el discriminante es por tanto:

$\Delta=T r^{2}-4 D e t<0$,

que expresado en función de los coeficientes de la matriz característica es:

$\left(A_{11}+A_{22}\right)^{2}+4\left(A_{12} A_{21}\right)<0$,

es decir:

$\frac{\left(A_{11}+A_{22}\right)^{2}}{4}<-A_{12} A_{21}$

Por tanto:

$$
A_{12} A_{21}<0,
$$

$\mathrm{y}$

$\frac{\left(A_{11}+A_{22}\right)^{2}}{4}<\left|A_{12} A_{21}\right|$

Para el presente caso la estabilidad estará garantizada si se cumple la condición de que la parte real de la raíz sea menor que cero: 
$\operatorname{Tr}=A_{11}+A_{22}<0$.

Se puede ver de (46) que en este caso la estabilidad se encuentra garantizada y que el parámetro $k_{31}$ refuerza con un orden de magnitud mayor la condición de estabilidad independientemente de su signo.

\subsubsection{Foco estable}

Este caso ocurre cuando las dos raíces son iguales con parte real negativa y por tanto el discriminante es:

$\Delta=T r^{2}-4 D e t=0$,

que expresado en función de los coeficientes de la matriz característica es:

$$
\begin{aligned}
& \left(A_{11}+A_{22}\right)^{2}+4\left(A_{12} A_{21}\right)=0, \\
& \left(A_{11}+A_{22}\right)^{2}=-4\left(A_{12} A_{21}\right) .
\end{aligned}
$$

Las condiciones de estabilidad para este caso estarán dadas por:

$\operatorname{Tr}=A_{11}+A_{22}<0$.

Por tanto las conclusiones que pueden sacarse sobre la contribución de $k_{31}$ en la estabilidad del sistema son idénticas al caso anterior, de espiral estable.

\subsubsection{Análisis de casos según los parámetros $N_{e}, k_{e}$}

En la dinámica del pararrotor el límite de las regiones estables está dado por la condición:

$$
\lambda_{1,2}=\frac{1}{2}\left(\operatorname{Tr} \pm \sqrt{\operatorname{Tr}^{2}-4 D e t}\right)=0
$$


En estudios anteriores de Nadal Mora (2005) se representaron las condiciones de estabilidad para distintos casos a partir de las variables $N_{e}$ y $k_{e}$. En el presente estudio se retoma la representación en función del parámetro $N_{e}$ ' (expresión (70)), caso más general de $N_{e}$, y $k_{e}$.

$k_{e}$ se define como una relación de momentos de inercia tal que:

$k_{e}=4 k_{21}^{2} \frac{I_{2}}{I_{1}} \frac{I_{3}-I_{1}}{I_{3}-I_{2}}$

Es posible expresar por tanto:

$\Delta=C^{2}\left(\operatorname{Tr}^{\prime 2}-k_{e}\left(1-N_{e}{ }^{\prime}\right)\right)$,

donde:

$C^{2}=\frac{\left(I_{3}-I_{2}\right)^{2}}{4 k_{21}} \frac{I_{1}}{I_{2}}$

Es posible observar que la constante $C$ será siempre mayor que 0 .

Retomando el límite impuesto por (87) tenemos que:

$\lambda_{1}=\frac{1}{2}\left(\operatorname{Tr} \pm \sqrt{\operatorname{Tr}^{2}-4 D e t}\right)=\frac{1}{2}\left(C \operatorname{Tr}^{\prime} \pm \sqrt{C^{2}\left(\operatorname{Tr}^{\prime 2}-k_{e}\left(1-N_{e}{ }^{\prime}\right)\right)}\right)=0$.

La ecuación anterior equivale a decir que:

$\operatorname{Tr}^{\prime 2}=\operatorname{Tr}^{\prime 2}-k_{e}\left(1-N_{e}{ }^{\prime}\right)$

es decir,

$k_{e}\left(1-N_{e}{ }^{\prime}\right)=0$,

por lo tanto,

$N_{e}^{\prime}=1$, 
que es el límite de las regiones estables.

Las condiciones que deberán cumplirse para que el sistema que describe a la dinámica del pararrotor sea estable son $\operatorname{Tr}<0$ de (62) y $N_{e}{ }^{\prime}<1$ de (63).

Se puede observar que $k_{31}$ aporta siempre a la estabilidad en el caso que la solución del sistema sea de nodo estable.

Para el análisis de estabilidad se estudian los casos siguientes, que resultan de expresar los resultados en el plano $\left(N_{e}, k_{e}\right)$, según se presentan en la Tabla 2.4.

Tabla 0.4 Casos de configuración geométrica. Contribución de $k_{31}$ a la estabilidad.

\begin{tabular}{|c|c|c|c|}
\hline \multicolumn{2}{|c|}{ Caso } & $\begin{array}{c}\text { Detalle del } \\
\text { caso }\end{array}$ & Estabilización \\
\hline A & $\begin{array}{c}\text { Estabilidad inercial } \\
\text { Eje principal mayor próximo al eje de giro }\end{array}$ & $I_{3}>I_{2}, I_{1}$ & $k_{31}>0$ \\
\hline B & $\begin{array}{c}\text { Estabilización aerodinámica } \\
\text { Eje principal intermedio próximo al eje de } \\
\text { giro }\end{array}$ & $I_{2}>I_{3}>I_{1}$ & $k_{31}>0$ \\
\hline C & $\begin{array}{c}\text { Eje principal intermedio próximo al eje de } \\
\text { giro }\end{array}$ & $I_{1}>I_{3}>I_{2}$ & $k_{31}<0$ \\
\hline D & Eje principal menor próximo al eje de giro & $I_{1}, I_{2}>I_{3}$ & $k_{31}<0$ \\
\hline
\end{tabular}

\subsubsection{Caso A - Estabilidad inercial. Eje principal mayor próximo al eje de giro $-I_{3}>$} $I_{2}, I_{1}$

En este caso, $I_{3}>I_{2}, I_{1}$; en consecuencia:

- $\quad$ para $k_{31}=0$, sólo es posible que $N_{e}{ }^{\prime}>0$, 
- $\quad$ para $k_{31} \neq 0$, las dos alternativas $N_{e}{ }^{\prime}<0$ o $N_{e}{ }^{\prime}>0$ son alcanzables. En consecuencia es accesible el primer y segundo cuadrante del plano $\left(N_{e}{ }^{\prime}, k_{e}\right)$.

La región que garantiza la estabilidad es $N_{e}{ }^{\prime}<1$ (tal cual se indica en la Figura 2.8).

Los distintos términos de $N_{e}$ ' contribuirán a la estabilidad si toman valores menores a 0 , y a la inestabilidad si toman valores mayores a 0 .

El análisis término a término realizado previamente del parámetro $N_{e}$, arroja las siguientes conclusiones para este caso:

- $\quad N_{e}{ }^{\prime}(i)<0$, para $k_{31}>0$,

- $\quad N_{e}^{\prime}($ (ii) $>0$.

Para el presente caso sólo es accesible el primer y segundo cuadrante del plano $\left(N_{e}{ }^{\prime}, k_{e}\right)$. A diferencia del caso cuando $k_{31}=0$, hay regiones donde $N_{e}{ }^{\prime}<0$ que son alcanzables, donde la distancia a los límites de estabilidad es mayor.

El límite de la región de espirales (focos) está dado por:

$$
\Delta=\operatorname{Tr}^{\prime 2}-C^{2} k_{e}\left(1-N_{e}{ }^{\prime}\right)=0
$$

$$
\Delta=C^{2}\left(\operatorname{Tr}^{\prime 2}-k_{e}\left(1-N_{e}{ }^{\prime}\right)\right)=0
$$

La región de sillas estará dada por:

$$
k_{e}>\frac{T r^{\prime 2}}{\left(1-N_{e}^{\prime}\right)}
$$

Estas condiciones pueden verse representadas en la Figura 2.5. Cuando $N_{e}{ }^{\prime}>1$ el sistema resulta inestable. 


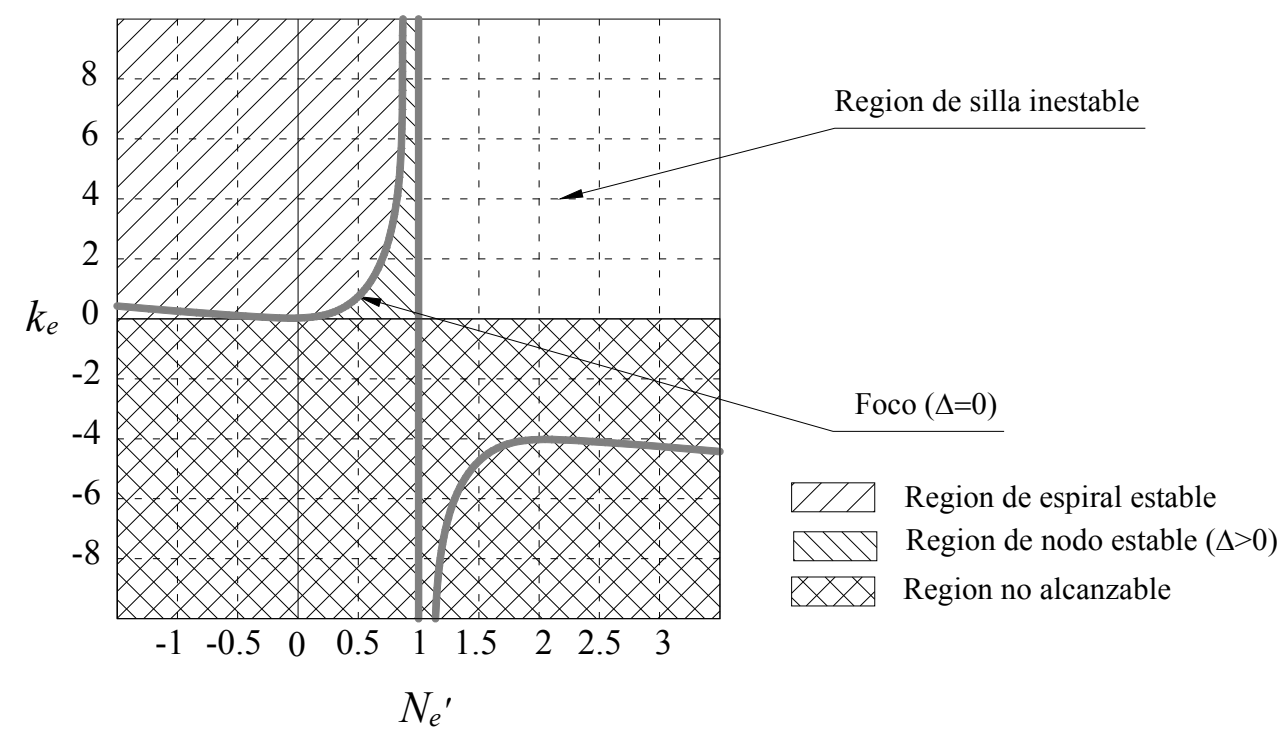

Figura 0.8 Caso A - Tipos de trayectorias en función de los valores de la traza $\operatorname{Tr}$ y del determinante Det de la ecuación característica, para $I_{3}>I_{2}, I_{1}$.

En el caso que $I_{3}>>I_{2}, I_{1}$, entonces $N_{e}$ 'tiende a 0 y el parámetro $k_{31}$ deja de ser relevante para la estabilidad del sistema.

\subsubsection{Caso B - Estabilización aerodinámica. Eje principal intermedio próximo al eje de giro $-I_{2}>I_{3}>I_{1}$}

En este caso, los momentos de inercia están ordenados de la siguiente forma: $I_{2}>I_{3}>I_{1}$.

De acuerdo a (69) debe ser $N_{e}{ }^{\prime}>1$ para que el sistema sea estable.

Para el caso que $k_{31}=0$, por definición $N_{e}$ ' $<0$ y $k_{e}<0$, y sólo es accesible el tercer cuadrante del plano $\left(N_{e}, k_{e}\right)$. Para el caso que $k_{31} \neq 0$ son accesibles el tercer y cuarto cuadrante.

Los distintos términos de $N_{e}$ ' contribuirán a la estabilidad si toman valores mayores a 0 , o a la inestabilidad si toman valores menores a 0 . 
Para este caso:

- $\quad N_{e}{ }^{\prime}(i)>0$, si $k_{31}>0$,

- $\quad N_{e}{ }^{\prime}(i i)<0$.

Se puede ver entonces que la contribución de $k_{31}$ depende de la relación de momentos de inercia. Cuando $k_{31}=0$ no es posible alcanzar una configuración estable.

Se observa que el primer término de $N_{e}$ ' aportará positivamente a la estabilidad para $k_{31}>0 \mathrm{y}$ no así el último término. Por lo tanto es posible alcanzar la región estable si $k_{21}>\frac{I_{3}-I_{2}}{I_{a} C L_{\alpha}}-k_{31}\left(\beta_{0}-2 k\right)$, para valores suficientemente grandes de $k_{31}>0$. Los resultados se muestran en la Figura 2.9.

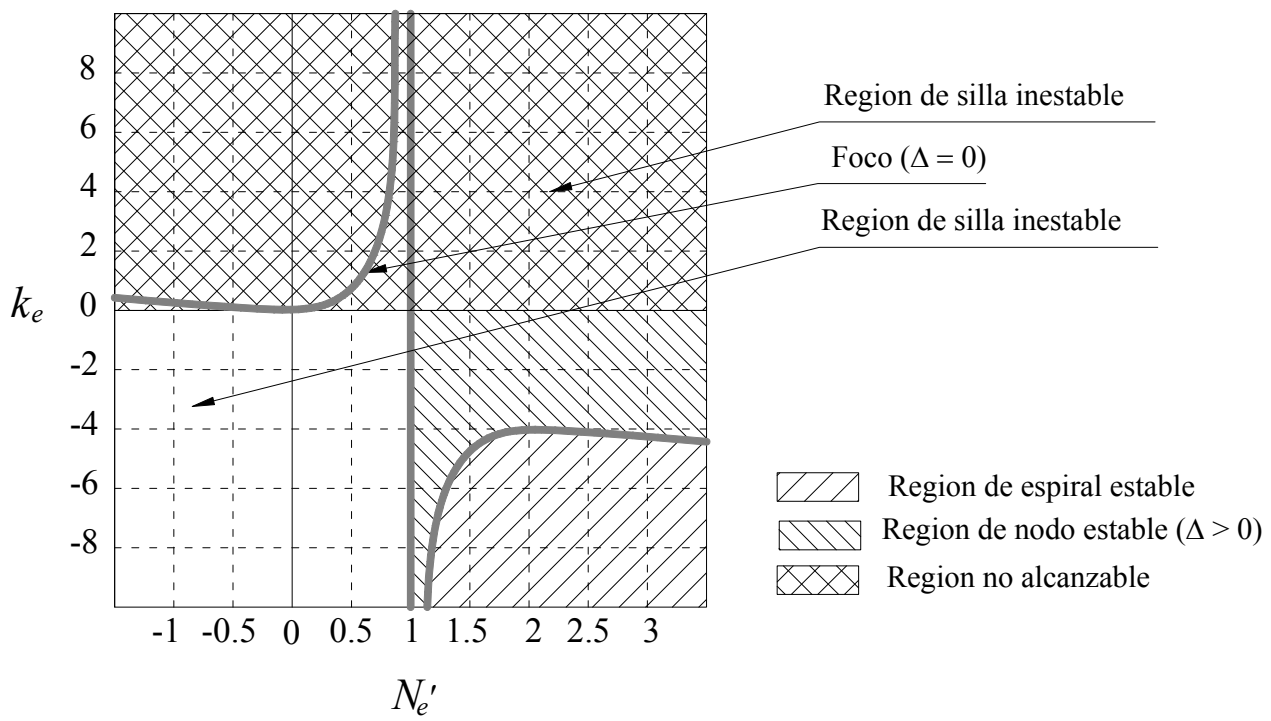

Figura 0.9 Caso B. Tipos de trayectorias en función de los valores de la traza $\operatorname{Tr}$ y del determinante Det de la ecuación característica, para $I_{2}>I_{3}>I_{1}$. 


\subsubsection{Caso C - Estabilización aerodinámica. Eje principal intermedio próximo al eje de} giro $-I_{1}>I_{3}>I_{2}$

En este caso, los momentos de inercia están ordenados de la siguiente forma: $I_{1}>I_{3}>I_{2}$.

De acuerdo a (69) debe ser $N_{e},>1$ para que el sistema sea estable.

Para el caso que $k_{31}=0$, por definición $N_{e}{ }^{\prime}>0$ y $k_{e}<0$, y sólo es accesible el cuarto cuadrante del plano $\left(N_{e}, k_{e}\right)$. Para el caso que $N_{e}{ }^{\prime} \neq 0$ son accesibles el tercer y cuarto cuadrante.

Los distintos términos de $N_{e}$, contribuyen a la estabilidad si toman valores mayores a 0 o a la inestabilidad si toman valores menores a 0 .

Para este caso:

- $\quad N_{e}^{\prime}(i)<0$, si $k_{31}>0$,

- $\quad N_{e}^{\prime}{ }_{(i i)}>0$.

Obsérvese que el primer término de $N_{e}$ ' contribuye negativamente a la estabilidad para $k_{31}>0$ y no así el último término. Por lo tanto es posible alcanzar la región estable si $k_{21}>\frac{I_{3}-I_{2}}{I_{a} C L_{\alpha}}-k_{31}\left(\beta_{0}-2 k\right)$. Esto equivale a decir que para pequeños valores positivos de $k_{31} \mathrm{y}$ para valores negativos de $k_{31}$ es posible alcanzar la estabilidad. Los resultados se muestran en la Figura 2.10. 


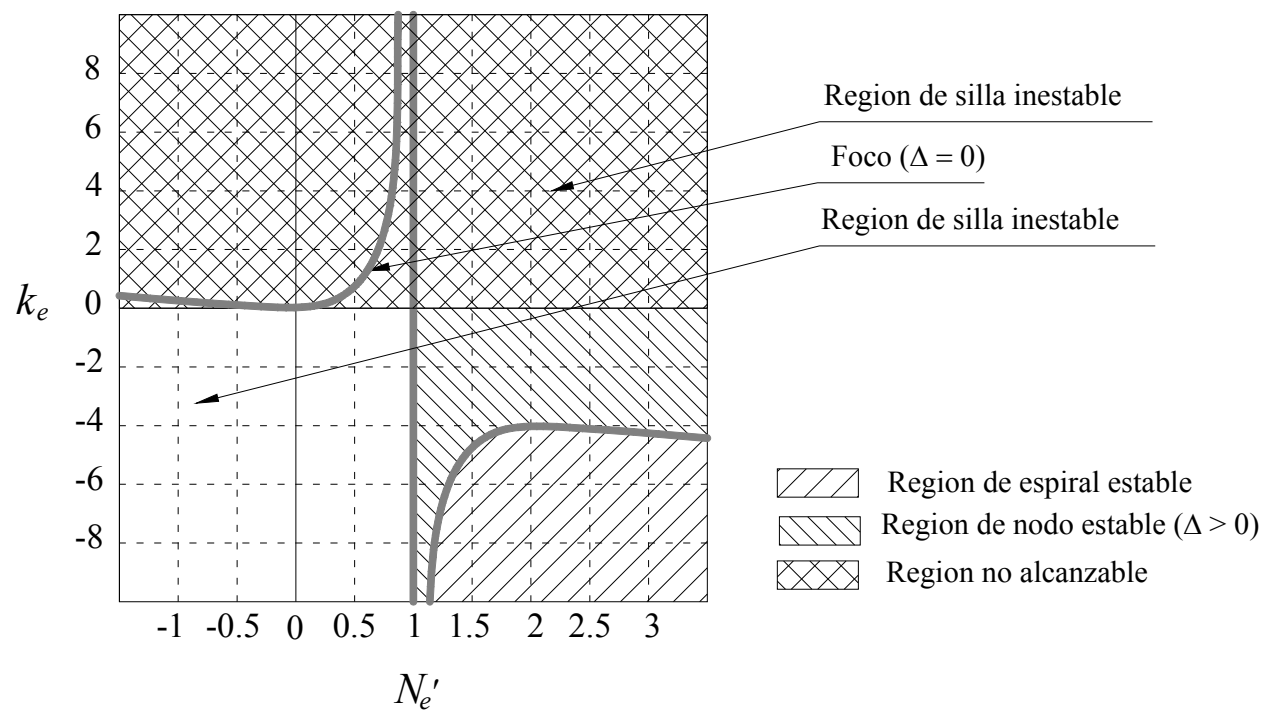

Figura 0.10 Caso C - Tipos de trayectoria en función de los valores de la traza $\operatorname{Tr}$ y del determinante Det de la ecuación carácterística, $I_{1}>I_{3}>I_{2}$.

\subsubsection{Caso D - Estabilidad inercial. Eje principal menor próximo al eje de giro - $I_{1}, I_{2}>$} $\boldsymbol{I}_{3}$

En este caso, $I_{1}, I_{2}>I_{3}$; en consecuencia necesariamente $k_{e}>0$. Para $k_{31}=0$, sucede que necesariamente es $N_{e}{ }^{\prime}<0$. Para $k_{31} \neq 0$ las alternativas $N_{e}{ }^{\prime}<0$ o $N_{e}{ }^{\prime}>0$ son alcanzables, dependiendo de los valores que tomen las variables que definen $N_{e}$. En consecuencia sólo es accesible el primer y segundo cuadrante del plano $\left(N_{e}{ }^{2}, k_{e}\right)$.

La región estable es $N_{e}{ }^{\prime}<1$ (como se indica en la Figura 2.11).

El análisis término a término realizado previamente del parámetro $N_{e}$ arroja las siguientes conclusiones para este caso:

- $\quad N_{e}{ }^{\prime}(i)>0$, para $k_{31}>0$

- $\quad N_{e}{ }^{\prime}(i i)>0$ 
Se puede observar que si $I_{3}<I_{2}, I_{1}$, entonces $k_{31}>0$ recorta los límites de estabilidad, y a la inversa $k_{31}<0$ aporta a la estabilidad.

Cuando $N_{e}{ }^{\prime}=0$, la evolución del sistema sigue una trayectoria tipo centro. Esta condición se corresponde con $C_{L \alpha}=0$, que es el caso de un sistema sin fuerzas aerodinámicas. En ese caso se demuestra que la disipación interna de energía mecánica hace que el giro alrededor del eje de menor inercia se vuelva inestable (Thomson, 1986). En caso de no tener en cuenta tal disipación de energía, el sistema sería estable para $N_{e}{ }^{\prime}=0$.

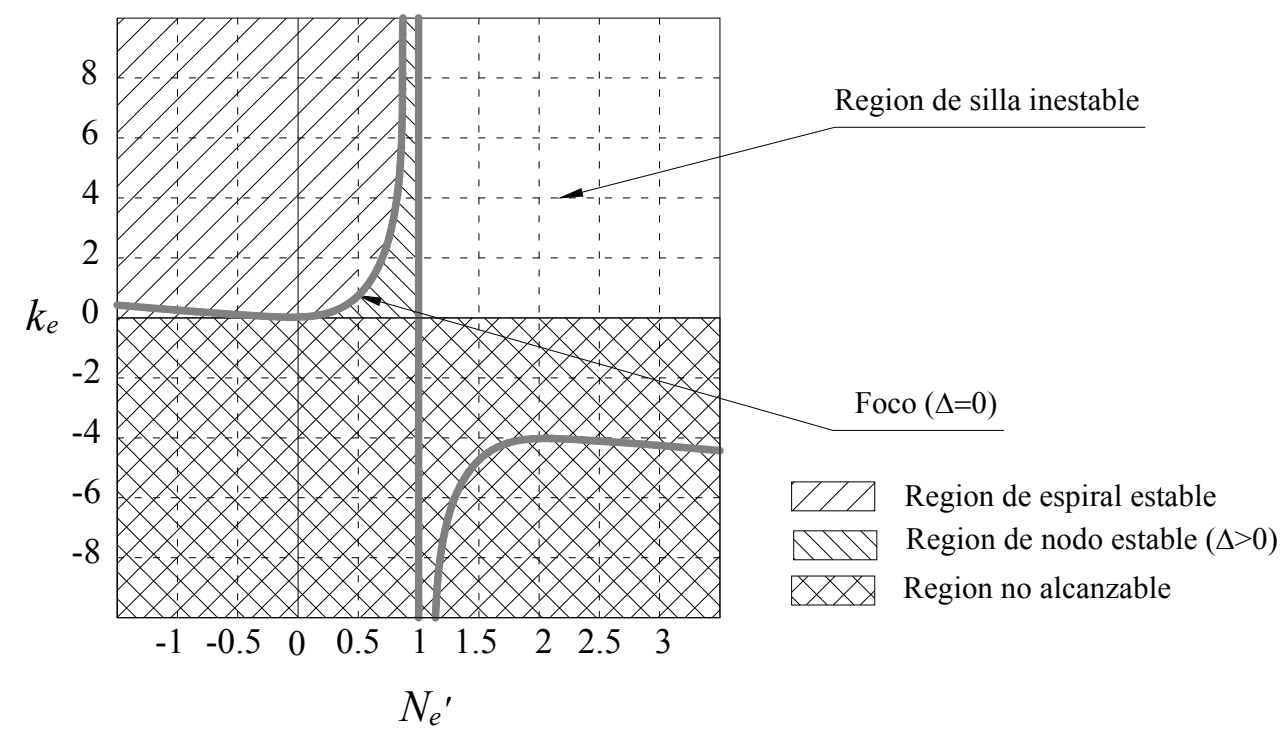

Figura 0.11 Caso D - Tipos de trayectorias en función de los valores de la traza $\operatorname{Tr}$ y del determinante Det de la ecuación característica, para $I_{1}, I_{2}>I_{3}$.

\subsection{ANGULO DE NUTACIÓN}

En el presente apartado se plantean las ecuaciones que permiten determinar los ángulos de nutación, $\theta$ (inclinación del eje 3 de la terna ejes cuerpo, respecto del eje $Z$ de la terna 
inercial), y de rotación propia, $\varphi$ (ángulo formado entre la proyección del eje $Z$ en el plano 1 -

2 y el eje 2), de acuerdo con la geometría mostrada en la Fig. 2.2.

Las componentes de la velocidad angular, $\bar{\omega}$, en ejes cuerpo son:

$\omega_{1}=\dot{\psi} \sin \theta \sin \varphi+\dot{\theta} \cos \varphi$

$\omega_{2}=\dot{\psi} \sin \theta \cos \varphi-\dot{\theta} \sin \varphi$

$\omega_{3}=\dot{\varphi}+\dot{\psi} \cos \theta$

En el caso estacionario no se observa movimiento de nutación $(\dot{\theta}=0)$, por lo tanto las ecuaciones se reducen a:

$\omega_{1}=\dot{\psi} \sin \theta \sin \varphi$

$\omega_{2}=\dot{\psi} \sin \theta \cos \varphi$

$\omega_{3}=\dot{\varphi}+\dot{\psi} \cos \theta$

Si se define $\omega_{12}=\sqrt{\omega_{1}^{2}+\omega_{2}^{2}}$. Combinando (a) y (b), se tiene que:

$\omega_{12}=\dot{\psi} \sin \theta$

y también se tiene:

$\frac{\omega_{1}}{\omega_{2}}=\tan \varphi$

En el caso estacionario $\omega_{1}=$ cte y $\omega_{2}=$ cte. Por lo tanto, de (97), se obtiene que:

$\varphi=$ cte $\rightarrow \dot{\varphi}=0$

$\mathrm{y}$, por lo tanto, de (95c), se deduce que: 
$\omega_{3}=\dot{\psi} \cos \theta$

De (96) y (95c), resulta que:

$$
\tan \theta=\frac{\omega_{12}}{\omega_{3}}
$$

\subsection{RESUMEN}

Para pequeños desplazamientos respecto del centro de masas del eje que contiene a las palas, la estabilidad del pararrotor (del modelo del sistema linealizado) no se ve afectada por el parámetro $k_{31}$, respecto del caso en que este eje pase por el centro de masas, bajo las hipótesis del modelo y las simplificaciones realizadas según los órdenes de magnitud de cada término (Piechocki, 2008). Sin embargo, esta distancia sí influye en la estabilidad dinámica del dispositivo, si es del orden de la distancia desde el centro de presiones de las palas al eje de revolución del pararrotor.

El parámetro $k_{31}$ podría utilizarse como parámetro de control del sistema, al igual que la diferencia entre los ángulos de paso de las palas.

La estabilidad del modelo simplificado se encuentra definida en mayor medida por la relación de los momentos de inercia.

Es posible tipificar la contribución del parámetro $k_{31}$ como se presenta en la Tabla 2.4.

\subsection{EJEMPLOS NUMÉRICOS}

En el presente apartado se consideran diversos ejemplos que contemplan los 4 casos presentados en el análisis de la estabilidad (Tabla 2.4), para comprobar, en términos numéricos resolviendo analíticamente las ecuaciones (45), (54), (55), sobre un configuración física concreta y posible, las conclusiones a las que se arribó. Estos ejemplos numéricos se 
realizan sobre una configuración básica definida por una serie de parámetros que permanecerán constantes, que se resumen en la Tabla 2.5.

Los ejemplos numéricos elegidos tienen por objeto mostrar en términos cuantitativos la respuesta dinámica de los casos predichos por el modelo analítico, sobre la base de valores de configuración de relevancia práctica. En particular se eligieron los siguientes cinco ejemplos, de mayor importancia práctica:

- Ejemplo numérico 1.1 - Caso $\mathrm{A}-I_{3}>I_{2}, I_{1}$ - Soluciones tipo nodo estable

- Ejemplo numérico 1.2 - Caso $\mathrm{A}-I_{3}>I_{2}, I_{1}$ - Soluciones tipo nodo estable y sillas inestables

- Ejemplo numérico 2 - Caso $\mathrm{B}-I_{2}>I_{3}>I_{1}$ - Soluciones tipo sillas inestables

- Ejemplo numérico 3 - Caso $\mathrm{C}-I_{1}>I_{3}>I_{2}$ - Soluciones tipo sillas inestables

- Ejemplo numérico 4 - Caso C $-I_{1}, I_{2}>I_{3}$ - Soluciones tipo nodo estable

Tabla 0.5 Parámetros generales del ejemplo numérico

\begin{tabular}{|c|c|c|}
\hline Parámetro & Valor & Dimensiones \\
\hline$C_{L \alpha}$ & 3,4 & {$[1 / \mathrm{rad}]$} \\
\hline$C_{D}$ & 0,07 & {$[-]$} \\
\hline$\rho$ & 1,21 & {$\left[\mathrm{~kg} / \mathrm{m}^{3}\right]$} \\
\hline$\omega_{0}$ & 291,4 & {$[\mathrm{rad} / \mathrm{s}]$} \\
\hline$S$ & 0,012 & {$\left[\mathrm{~m}^{2}\right]$} \\
\hline$r_{i 1}$ & 0,1 & {$[\mathrm{~m}]$} \\
\hline$r_{i 2}$ & 0,034 & {$[\mathrm{~m}]$} \\
\hline$\beta_{1}$ & 0,1 & {$[\mathrm{rad}]$} \\
\hline$\beta_{2}$ & 0,17 & {$[\mathrm{rad}]$} \\
\hline
\end{tabular}




\begin{tabular}{|c|c|c|}
\hline Parámetro & Valor & Dimensiones \\
\hline$\delta_{\beta}$ & 0,035 & {$[\mathrm{rad}]$} \\
\hline$U_{\infty}$ & 14,1 & {$[\mathrm{~m} / \mathrm{s}]$} \\
\hline
\end{tabular}

Los ejemplos numéricos que se consideran incluyen los resultados de sus correspondientes soluciones de equilibrio y autovalores, para 5 valores del factor $k_{31}$ :

$$
\begin{aligned}
& k_{31}=-1 \\
& k_{31}=-0,5 \\
& k_{31}=0 \\
& k_{31}=0,5 \\
& k_{31}=1
\end{aligned}
$$

En las tablas que siguen se considerarán los autovalores de acuerdo a la siguiente expresión:

$$
\begin{aligned}
& \lambda_{1}=\lambda_{A 1}+\sqrt{\lambda_{B 1}}=\lambda_{r e 1}+i \lambda_{i m 1} \\
& \lambda_{2}=\lambda_{A 2}-\sqrt{\lambda_{B 2}}=\lambda_{r e 2}-i \lambda_{i m 2}
\end{aligned}
$$

Las condiciones de estabilidad del sistema se resumen en la Tabla 2.6.

Tabla 0.6 Condiciones generales de estabilidad asintótica para la evaluación de los ejemplos numéricos

\begin{tabular}{|c|c|}
\hline \multicolumn{2}{|c|}{ Condiciones de estabilidad } \\
\hline$\lambda_{r e i}<0 ; i=1,2$ & Estabilidad \\
\hline$\lambda_{r e 1}=0$ & Estabilidad \\
$\lambda_{r e 2}<0$ & Inestabilidad \\
\hline$\lambda_{r e i} \geq 0 ; i=1,2$ & \\
\hline
\end{tabular}


En la Tabla 2.7 se presentan las soluciones para el ejemplo numérico 1.1, donde $I_{3}>I_{2}, I_{1}$, y el comportamiento dinámico es del tipo nodo estable.

Tabla 0.7 Caso A - Estabilidad inercial $-I_{3}>I_{2}, I_{1}$. Solución tipo nodo estable.

\begin{tabular}{|c|c|c|c|c|c|}
\hline $\begin{array}{r}\text { Eje } p \\
I_{1}=6,31 \cdot 10\end{array}$ & ${ }^{-4} \mathrm{~kg} \mathrm{~m}^{2}$ & $\begin{array}{r}\text { Ejemplo } \\
\text { Estabili } \\
\text { hayor pró } \\
I_{2}=22,0\end{array}$ & $\begin{array}{l}\text { Imérico } \\
\mathrm{d} \text { inercia } \\
\text { mo al eje } \\
0^{-4} \mathrm{~kg} \mathrm{~m}^{2}\end{array}$ & $\begin{array}{l}\text { giro } I_{3}> \\
3=26,32\end{array}$ & ${ }^{I_{1}} \mathrm{~kg} \mathrm{~m}^{2}$. \\
\hline & & Par & netros & & \\
\hline$k_{31}$ & -1 & $-0,5$ & 0 & 0,5 & 1 \\
\hline & Resu & Itado nu & rico del & delo & \\
\hline$x_{e q 1}$ & $-0,0026$ & $-0,0024$ & $-0,0022$ & $-0,0021$ & $-0,0020$ \\
\hline$x_{e q 2}$ & $-0,0112$ & $-0,0069$ & $-0,0036$ & $-0,0009$ & 0,0012 \\
\hline$\lambda_{r e 1}=\lambda_{r e 2}$ & $-0,0130$ & $-0,0118$ & $-0,0114$ & $-0,0118$ & $-0,0130$ \\
\hline$\lambda_{i m 1}=-\lambda_{i m 2}$ & 0,7236 & 0,7456 & 0,7669 & 0,7876 & 0,8078 \\
\hline$N_{e}^{\prime}$ & 0,14 & 0,09 & 0,04 & $-0,01$ & $-0,07$ \\
\hline$k_{e}$ & 7,59 & 7,59 & 7,59 & 7,59 & 7,59 \\
\hline
\end{tabular}

En la Tabla 2.7 se observa que el sistema tiene sus autovalores complejos conjugados con parte real negativa, y por tanto resulta en soluciones del tipo nodo estable. En este caso se puede ver que el factor $k_{31}$, independientemente de su signo, contribuye a la estabilidad haciendo menor el valor de la parte real de los autovalores. No obstante $N_{e}$ ' se hace más negativo conforme aumenta $k_{31}$ (con $k_{e}$ constante) el punto representativo en el plano $N_{e}$ - $k_{e}$ se aleja del límite de estabilidad $\left(N_{e}{ }^{\prime}=1\right)$ y por lo tanto aumenta su contribución a la estabilidad.

En la Tabla 2.8 se presentan las soluciones para el ejemplo numérico 1.2 donde $I_{3}>I_{2}, I_{1}$, donde el comportamiento dinámico muestra regiones de inestabilidad. Se observa que los autovalores del sistema son números complejos conjugados con parte real negativa para valores de $k_{31}>0$, y por tanto resulta en soluciones del tipo nodo estable. También se presentan autovalores reales, que devienen en soluciones tipo silla inestable para valores de 
$k_{31}<0$. En este caso se puede ver que el factor $k_{31}$ aporta a la estabilidad positivamente para valores crecientes de $k_{31}$, y vale decir que el desplazamiento positivo del plano que contiene a las palas por encima el centro de masas contribuye a la estabilidad del sistema como en el caso anterior.

Tabla 0.8 Caso A - Estabilidad inercial $-I_{3}>I_{2}, I_{1}$. Solución tipo nodo estable/sillas inestables.

\begin{tabular}{|c|c|c|c|c|c|}
\hline \multicolumn{6}{|c|}{$\begin{array}{l}\text { Ejemplo numérico } 1.2 \\
\quad \text { Estabilidad inercial. } \\
\text { mayor próximo al eje de giro } I_{3}>I_{2}, I_{1} \\
, I_{2}=22,06 \cdot 10^{-4} \mathrm{~kg} \mathrm{~m}^{2}, I_{3}=22,5 \cdot 10^{-4} \mathrm{~kg} \mathrm{~m}^{2} \text {. }\end{array}$} \\
\hline \multicolumn{6}{|c|}{ Parámetros } \\
\hline$k_{31}$ & -1 & $-0,5$ & 0 & 0,5 & 1 \\
\hline \multicolumn{6}{|c|}{ Resultado numérico del modelo } \\
\hline$x_{e q 1}$ & $-0,0003$ & 0,0001 & 0,0029 & $-0,0030$ & $-0,0017$ \\
\hline$x_{e q 2}$ & 0,0294 & 0,0361 & 0,0806 & $-0,0128$ & 0,0073 \\
\hline$\lambda_{r e} 1$ & 0,1984 & 0,1336 & $-0,0114$ & $-0,0118$ & $-0,0130$ \\
\hline$\lambda_{i m 1}$ & 0,0000 & 0,0000 & 0,0491 & 0,1610 & 0,2224 \\
\hline$\lambda_{\text {re } 2}$ & $-0,2245$ & $-0,1572$ & $-0,0114$ & $-0,0118$ & $-0,0130$ \\
\hline$\lambda_{i m 2}$ & 0,0000 & 0,0000 & $-0,0491$ & $-0,1610$ & $-0,2224$ \\
\hline$N_{e}^{\prime}$ & 3,05 & 1,97 & 0,88 & $-0,20$ & $-1,28$ \\
\hline$k_{e}$ & 135,62 & 135,62 & 135,62 & 135,62 & 135,62 \\
\hline
\end{tabular}

Se observa en la Tabla 2.9, donde se muestran resultados para el caso B $\left(I_{2}>I_{3}>I_{1}\right)$, que el sistema resulta inestable para los valores de $k_{31}$ presentados. Los autovalores del sistema para estos casos son reales, donde uno de ellos es mayor que cero. El desplazamiento del plano que contiene a las palas por encima el centro de masas disminuye la magnitud positiva de dicho autovalor que resulta mayor que cero. 
Tabla 0.9 Caso B - Estabilización aerodinámica $-I_{2}>I_{3}>I_{1}$. Solución tipo sillas inestables.

\begin{tabular}{|c|c|c|c|c|c|}
\hline \multicolumn{6}{|c|}{$\begin{array}{l}\text { Ejemplo numérico } 2 \\
\text { stabilización aerodinámica } \\
\qquad I_{2}>I_{3}>I_{1} \\
I_{2}=26,32 \cdot 10^{-4} \mathrm{~kg} \mathrm{~m}^{2}, I_{3}=22,06 \cdot 10^{-4} \mathrm{~kg} \mathrm{~m}^{2} .\end{array}$} \\
\hline \multicolumn{6}{|c|}{ Parámetros } \\
\hline$k_{31}$ & -1 & $-0,5$ & 0 & 0,5 & 1 \\
\hline \multicolumn{6}{|c|}{ Resultado numérico del modelo } \\
\hline$x_{e q 1}$ & $-0,0022$ & $-0,0023$ & $-0,0024$ & $-0,0025$ & $-0,0027$ \\
\hline$x_{e q 2}$ & 0,0061 & 0,0047 & 0,0030 & 0,0010 & $-0,0016$ \\
\hline$\lambda_{r e} 1$ & 0,6690 & 0,6543 & 0,6385 & 0,6215 & 0,6032 \\
\hline$\lambda_{i m 1}=\lambda_{i m 2}$ & 0,0000 & 0,0000 & 0,0000 & 0,0000 & 0,0000 \\
\hline$\lambda_{\text {re } 2}$ & $-0,6913$ & $-0,6743$ & $-0,6576$ & $-0,6414$ & $-0,6256$ \\
\hline$N_{e}^{\prime}$ & $-0,14$ & $-0,09$ & $-0,04$ & 0,01 & 0,07 \\
\hline$k_{e}$ & $-7,13$ & $-7,13$ & $-7,13$ & $-7,13$ & $-7,13$ \\
\hline
\end{tabular}

En el ejemplo que se presenta en la Tabla 2.10, donde se muestran resultados para el caso C $\left(I_{1}>I_{3}>I_{2}\right)$, el sistema resulta inestable para los valores de $k_{31}$ considerados. Los autovalores del sistema para estos casos son reales, donde uno de ellos es mayor que cero. El desplazamiento del plano que contiene a las palas por debajo del centro de masas (valores negativos de $k_{31}$ ) aumenta la magnitud positiva de dicho autovalor.

Tabla 0.10 Caso C - Estabilización aerodinámica $-I_{1}>I_{3}>I_{2}$. Solución tipo sillas inestables.

\begin{tabular}{|c|c|c|c|c|c|}
\hline \multicolumn{6}{|c|}{$\begin{array}{c}\text { Ejemplo numérico 3 } \\
\text { Estabilización aerodinámica } \\
\qquad I_{1}>I_{3}>I_{2} \\
\mathrm{~kg} \mathrm{~m}^{2}, I_{2}=6,31 \cdot 10^{-4} \mathrm{~kg} \mathrm{~m}^{2}, I_{3}=22,06 \cdot 10^{-4} \mathrm{~kg} \mathrm{~m}^{2} .\end{array}$} \\
\hline \multicolumn{6}{|c|}{ Parámetros } \\
\hline$k_{31}$ & -1 & $-0,5$ & 0 & 0,5 & 1 \\
\hline \multicolumn{6}{|c|}{ Resultado numérico del modelo } \\
\hline$x_{e q 1}$ & 0,0102 & 0,0100 & 0,0098 & 0,0097 & 0,0095 \\
\hline
\end{tabular}




\begin{tabular}{|c|c|c|c|c|c|}
\hline \multicolumn{6}{|c|}{$\begin{array}{c}\text { Ejemplo numérico } 3 \\
\text { Estabilización aerodinámica } \\
\quad I_{1}>I_{3}>I_{2} \\
\mathrm{~g} \mathrm{~m}^{2}, I_{2}=6,31 \cdot 10^{-4} \mathrm{~kg} \mathrm{~m}^{2}, I_{3}=22,06 \cdot 10^{-4} \mathrm{~kg} \mathrm{~m}^{2}\end{array}$} \\
\hline \multicolumn{6}{|c|}{ Parámetros } \\
\hline$k_{31}$ & -1 & $-0,5$ & 0 & 0,5 & 1 \\
\hline \multicolumn{6}{|c|}{ Resultado numérico del modelo } \\
\hline$x_{e q 2}$ & $-0,0023$ & $-0,0016$ & $-0,0009$ & $-0,0003$ & 0,0004 \\
\hline$\lambda_{r e} 1$ & 0,5840 & 0,5889 & 0,5935 & 0,5980 & 0,6022 \\
\hline$\lambda_{i m 1}=\lambda_{\text {im } 2}$ & 0,0000 & 0,0000 & 0,0000 & 0,0000 & 0,0000 \\
\hline$\lambda_{\text {re } 2}$ & $-0,6646$ & $-0,6689$ & $-0,6734$ & $-0,6780$ & $-0,6828$ \\
\hline$N_{e}^{\prime}$ & 0,04 & 0,02 & 0,01 & 0,00 & $-0,02$ \\
\hline$k_{e}$ & $-0,03$ & $-0,03$ & $-0,03$ & $-0,03$ & $-0,03$ \\
\hline
\end{tabular}

En el caso presentado en la Tabla 2.11, donde se presentan soluciones para $I_{1}, I_{2}>I_{3}$, se observa que los autovalores del sistema son complejos conjugados, y por tanto resultan en soluciones del sistema del tipo nodo estable. En este caso se puede ver que el factor $k_{31}$ contribuye a la estabilidad independientemente de su signo, haciendo que la parte real negativa de los autovalores del sistema aumente en magnitud. No obstante se verifica que este efecto de esta contribución es menor.

Tabla 0.11 Caso D - Estabilidad inercial $-I_{2}, I_{1}>I_{3}$. Solución tipo nodo estable.

\begin{tabular}{|c|c|c|c|c|c|}
\hline \multicolumn{7}{|c|}{$\begin{array}{c}\text { Ejemplo numérico } 4 \\
\text { Estabilidad inercial } \\
I_{1}, I_{2}>I_{3}\end{array}$} \\
$I_{1}=22,06 \cdot 10^{-4} \mathrm{~kg} \mathrm{~m}^{2}, I_{2}=26,32 \cdot 10^{-4} \mathrm{~kg} \mathrm{~m}^{2}, I_{3}=6,31 \cdot 10^{-4} \mathrm{~kg} \mathrm{~m}^{2}$. \\
\hline \multicolumn{7}{|c|}{ Parámetros } \\
\hline$k_{31}$ & -1 & $-0,5$ & 0 & 0,5 & 1 \\
\hline \multicolumn{7}{|c|}{ Resultado numérico del modelo } \\
\hline$x_{\text {eq } 1}$ & $-0,0021$ & $-0,0021$ & $-0,0022$ & $-0,0022$ & $-0,0022$ \\
\hline$x_{\text {eq2 }}$ & $-0,0013$ & $-0,0010$ & $-0,0006$ & $-0,0002$ & 0,0003 \\
\hline
\end{tabular}




\begin{tabular}{|c|c|c|c|c|c|}
\hline$\lambda_{\text {re } 1}=\lambda_{\text {re } 2}$ & $-0,0100$ & $-0,0097$ & $-0,0096$ & $-0,0097$ & $-0,0100$ \\
\hline$\lambda_{\text {im } 1}=-\lambda_{\text {im } 2}$ & 0,7473 & 0,7435 & 0,7398 & 0,7360 & 0,7322 \\
\hline$N_{e}^{\prime}$ & $-0,0289$ & $-0,0187$ & $-0,0084$ & 0,0019 & 0,0122 \\
\hline$k_{e}$ & 0,43 & 0,43 & 0,43 & 0,43 & 0,43 \\
\hline
\end{tabular}

Puede verse en la Figura 2.9 que tanto en el Ejemplo 1.1 como el Ejemplo 4 existe estabilidad. Si bien existe en estos ejemplos influencia del parámetro $k_{31}$ en la estabilidad, la magnitud de este efecto es limitada. En ambos ejemplos se trata de soluciones tipo nodo estable.

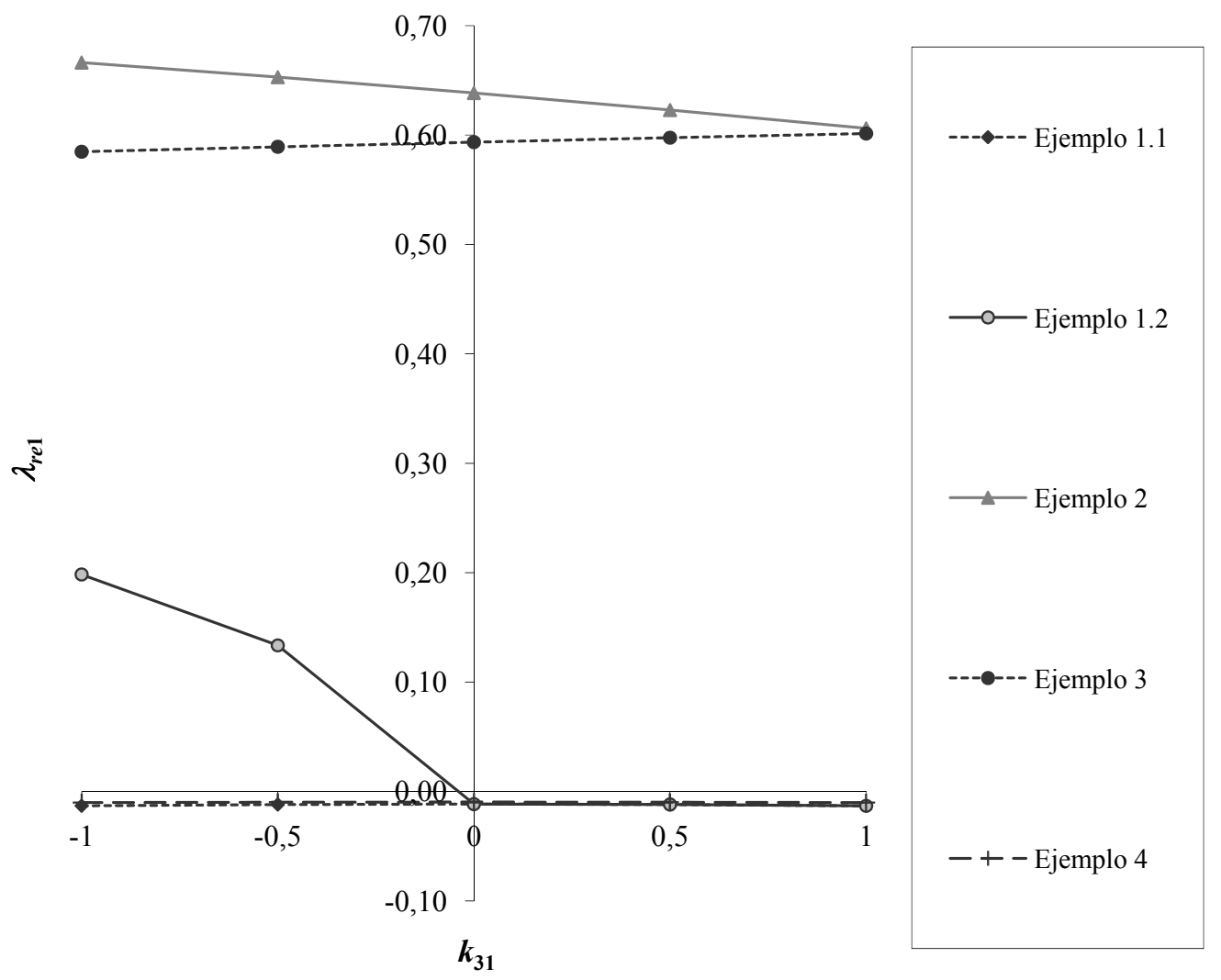

Figura 0.12 Variación del autovalor de parte real de mayor del sistema de ecuaciones que describe la estabilidad del sistema, con el parámetro $k_{31}$. 
Por otro lado puede observarse que para el Ejemplo 1.2, el efecto de $k_{31}$ para el comportamiento dinámico del sistema es determinante para su estabilidad, especialmente si la parte imaginaria de los autovalores son cercanos a 0 , lo que equivale a decir que el discriminante $\Delta$ es próximo a 0 (solución tipo foco). Vale decir que cuando la configuración del pararrotor se encuentra en las cercanías de las regiones inestables los efectos generados por el parámetro $k_{31}$ son determinantes de la respuesta dinámica del sistema.

\subsection{CONCLUSIÓN}

De acuerdo al modelo analítico propuesto, la estabilidad del pararrotor se encuentra definida en mayor medida por la relación de los momentos de inercia del dispositivo.

Para pequeños desplazamientos respecto del centro de masas del eje que contiene a las palas, la estabilidad del pararrotor (según el modelo linealizado del sistema) no se ve afectada por el parámetro $k_{31}$, respecto del caso en que este eje pase por el centro de masas, bajo las hipótesis del modelo y las simplificaciones realizadas según los órdenes de magnitud de cada término.

Para desplazamientos del orden de la distancia desde el centro de presiones de las palas al eje de revolución del pararrotor o mayores, estos sí influyen en la estabilidad dinámica del dispositivo.

En los Casos A y D es posible obtener configuraciones estables del desacelerador aerodinámico. En ambos casos valores positivos del parámetro $k_{31}$ (centro de masas por debajo del plano que contiene a las palas) contribuyen a la estabilidad del sistema.

En el caso A, el parámetro $k_{31}$ contribuye a la estabilidad si este es mayor que cero, aunque la magnitud de esta contribución es menor. En el caso que se esté cerca del límite estabilidad del sistema este parámetro resulta definitorio de la estabilidad. La presente relación de momentos de inercia resulta el caso de mayor aplicación práctica.

En el Caso B, el pararrotor se comporta de manera inestable, sin que los efectos del parámetro $k_{31}$ resulten determinantes. 
En el Caso C, el pararrotor se comporta en general de manera inestable. No obstante es posible alcanzar zonas de estabilidad para valores muy grandes de $k_{31}$.

En el Caso D el parámetro $k_{31}$ contribuye a la estabilidad tanto si este es mayor que cero como si este es menor que cero.

El parámetro $k_{31}$ podría utilizarse como parámetro de control del sistema, al igual que la diferencia entre los ángulos de paso de las palas. Este hecho se verifica al observar los términos independientes de la linealización del modelo matemático del sistema (39) y (41) que incluyen estos parámetros. Su utilización podría utilizarse para el guiado del dispositivo con velocidades de respuesta controladas. En cualquier caso ambos parámetros resultan parámetros de diseño determinantes. En términos prácticos si la distancia $k_{31}$ ajustara con el diedro de la pala, el control de los ángulos de inclinación de la pala en los ejes cuerpo 1 y 2 servirían para la regulación de ambos parámetros.

El ejemplo numérico confirma y aclara el efecto previsto de $k_{31}$ a la estabilidad del sistema al que se llega como conclusión del estudio analítico.

Es posible determinar los ángulos de nutación $\theta$ y de rotación propia $\phi$ de acuerdo con la geometría mostrada en la Figura 2.2 de idéntica manera a lo desarrollado para $k_{31}=0$ por Nadal Mora (2005) sobre la base de la descomposición de las velocidad angular en coordenadas móviles sobre la terna móvil. 


\section{CAPITULO 3}

\section{ESTUDIO DEL EFECTO DEL DESPLAZAMIENTO DEL PLANO QUE CONTIENE A LAS PALAS CON RESPECTO AL CENTRO DE MASAS A PARTIR DEL DESARROLLO DE UNA RUTINA DE SIMULACIÓN DE LA DINÁMICA DEL PARARROTOR}

\subsection{INTRODUCCIÓN}

La metodología adoptada para el estudio del efecto del desplazamiento del plano que contiene a las palas al respecto del centro de masas en un pararrotor ha sido plantear las ecuaciones de la dinámica del pararrotor en su formulación completa para su cómputo numérico sobre casos específicos. Posteriormente se ha estudiado la estabilidad dinámica del sistema considerando el efecto que resulta del desplazamiento del centro de masas al respecto del plano que contiene a las palas en conjunto con parámetros geométricos y aerodinámicos.

Se adopta como herramienta de cálculo el programa Scicos (similar al Simulink), el cual permite expresar en forma mimética (imitando circuitos analógicos) las distintas expresiones matemáticas de la formulación matemática. A partir de la creación de un modelo gráfico del sistema matemático es posible simular numéricamente el sistema.

El modelo gráfico del sistema se basa en tres tipos de elementos: entradas, bloques de proceso, y salidas. Este modelo gráfico describe relaciones matemáticas sobre una base temporal de flujo de datos. La representación gráfica dispone en el extremo izquierdo las 
entradas (numeradas) del proceso al cual se hace referencia y en el extremo derecho las salidas (numeradas).

La simulación numérica supone el establecimiento de un determinado intervalo de tiempo, y determinadas condiciones iniciales, sobre las cuales se computa el modelo matemático.

\subsection{MODELO MATEMÁTICO}

El sistema a estudiar corresponde a un dispositivo de vuelo rotatorio en régimen de autorrotación compuesto por un cuerpo cilíndrico con alas de pequeño alargamiento compuestas por placas planas, como se indica en la Figura 2.1.

A los fines de la elaboración del modelo matemático se considera un cuerpo que cae a velocidad uniforme $\mathbf{V}_{v}$ según la vertical y girando con velocidad $\omega$. El sistema ejes cuerpo, solidario al pararrotor, se designa por $1,2,3$, con direcciones $\mathbf{e}_{1}, \mathbf{e}_{2}, \mathbf{e}_{3}$ y origen situado en el centro de masas.

El sistema de referencia inercial es $X, Y, Z$, cuyos ejes poseen las direcciones $\mathbf{i}, \mathbf{j}$, k. La geometría del pararrotor está definida en la Figura 3.1. 


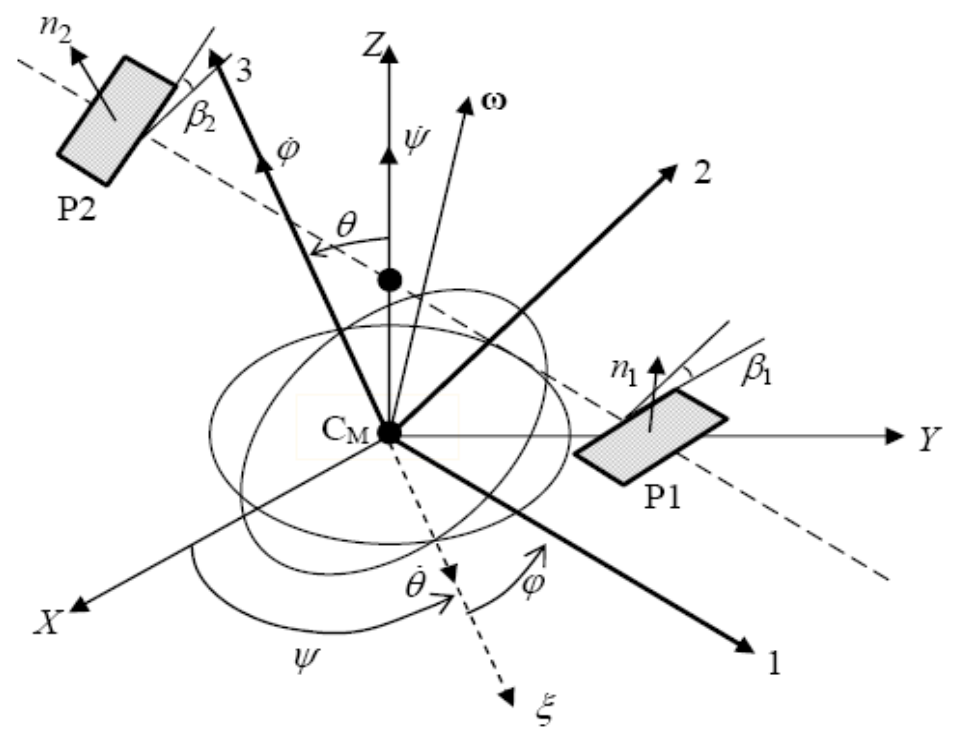

Figura 0.1 Definición de la geometría del pararrotor. $\psi$, precesión; $\theta$, nutación; $\varphi$, rotación propia.

Para la simulación numérica de la dinámica del pararrotor se utilizan cuaterniones normalizados para la definición de la orientación relativa de los ejes cuerpo al respecto de los ejes inerciales. A su vez, se transforman estos cuaterniones en ángulos de Euler para la representación de la orientación del pararrotor. Este proceso se realiza a partir de una matriz de transformación para el paso de coordenadas y vectores de un sistema a otro, de acuerdo con las expresiones para la representación de la actitud compendiadas por Diebel (2006).

El desarrollo del modelo matemático comprende:

- el planteo de la representación de los ejes móviles al respecto de los inerciales,

- el planteo de las ecuaciones de la dinámica del pararrotor,

- el planteo de las expresiones de fuerzas y momentos aerodinámicos de forma completa, introduciendo el efecto del desplazamiento de centro de masas al respecto del plano formado por las palas, 
- el planteo del modelo gráfico de las expresiones matemáticas que se usan como fuente de simulación.

\subsubsection{Orientación del pararrotor}

La representación espacial de la orientación del pararrotor supone contar con un medio para transformar los vectores relativos a los ejes adheridos al cuerpo en vectores referidos a la terna inercial.

Usualmente, la representación de la actitud de un cuerpo rígido se realiza por medio de tres ángulos y sus derivadas, como es el caso de los ángulos de Euler. El uso de esta parametrización deriva en la determinación de una matriz de transformación.

Es posible demostrar que el uso a estos fines de tres ángulos, como son los parámetros de Euler, supone la presencia de singularidades en la representación. Específicamente estas singularidades se presentan a valores de nutación $\theta=n \pi$, para $n=0,1,2,3 \ldots$ En estas orientaciones singulares en el espacio, los cambios en precesión y rotación propia constituyen el mismo movimiento. Estas singularidades (llamadas "gimbal lock" haciendo referencia a soportes cardánicos físicos) se explicitan en las ecuaciones diferenciales de la cinemática. En particular, la orientación del pararrotor que tomaremos como punto de partida de nuestros estudios se encuentra en el entorno de dicha singularidad.

El uso de cuaterniones para la representación espacial permite expresar la orientación relativa de un cuerpo rígido al respecto de un sistema inercial de referencia mediante el uso de un escalar y tres coordenadas vectoriales, eliminando todo tipo de singularidades. El uso de cuaterniones de orientación del cuerpo rígido permite obtener matrices de rotación, las que nos permitirán transformar coordenadas y vectores del sistema inercial de referencia al sistema de ejes cuerpo.

Se adoptará un cuaternión de orientación de norma 1, que tendrá la forma:

$$
\mathbf{q}=q_{0}+q_{1} \mathbf{e}_{1}+q_{2} \mathbf{e}_{2}+q_{3} \mathbf{e}_{3} .
$$


Es posible interpretar físicamente esta representación matemática como una rotación $q_{0}$ alrededor del vector dado por la expresión:

$$
q_{1} \mathbf{e}_{1}+q_{2} \mathbf{e}_{2}+q_{3} \mathbf{e}_{3}
$$

La derivada de este cuaternión con respecto al tiempo está dada, según Diebel (2006), por:

$$
\frac{\partial \mathbf{q}}{\partial t}=\frac{1}{2}\left[\begin{array}{c}
q_{1} \omega_{1}+q_{2} \omega_{2}+q_{3} \omega_{3} \\
q_{0} \omega_{1}+q_{2} \omega_{3}-q_{3} \omega_{2} \\
q_{0} \omega_{2}+q_{3} \omega_{1}-q_{1} \omega_{3} \\
q_{0} \omega_{3}+q_{1} \omega_{2}-q_{2} \omega_{1}
\end{array}\right]
$$

Para el cómputo de la orientación del pararrotor en el lapso de tiempo en que es analizada la dinámica del movimiento rotatorio del pararrotor, se integra la ecuación anterior sobre pasos de tiempo diferenciales. La orientación del pararrotor tendrá un punto de partida en el inicio del período de integración, definido por tres ángulos de Euler (que se equivalen a un cuaternión inicial) para la obtención de un cuaternión de orientación para cada punto en el tiempo de integración (o para cada paso de integración del cálculo numérico).

\subsubsection{Ortonormalización}

Las componentes de los cuaterniones no son todos independientes, pero satisfacen la ecuación:

$q_{0}^{2}+q_{1}^{2}+q_{2}^{2}+q_{3}^{2}=1$

de la cual derivando con respecto al tiempo se tiene:

$q_{0} q_{0}{ }^{\prime}+q_{1} q_{1}{ }^{\prime}+q_{2} q_{2}{ }^{\prime}+q_{3} q_{3}{ }^{\prime}=0$.

Las operaciones aritméticas sobre los cuaterniones introducirán errores numéricos que devendrán a lo largo de la integración de (104) en una distorsión acumulada que degradará la ortonormalidad del elemento. Este error puede computarse como: 
$\varepsilon=1-\left(q_{0}^{2}+q_{1}^{2}+q_{2}^{2}+q_{3}^{2}\right)$.

Por lo tanto su derivada se presenta como:

$q_{0} q_{0}{ }^{\prime}+q_{1} q_{1}{ }^{\prime}+q_{2} q_{2}{ }^{\prime}+q_{3} q_{3}{ }^{\prime}=\frac{-\varepsilon^{\prime}}{2}$.

El método que se adopta para reducir el error por no ortonormalidad es algebraico, de acuerdo a lo propuesto por Ai Chun Fang \& Zimmerman (1969). Este se basa en la suma para cada paso de integración de un término adicional en la ecuación (104), dado por:

$\frac{\partial \mathbf{q}}{\partial t}=\frac{1}{2}\left[\begin{array}{c}q_{1} \omega_{1}+q_{2} \omega_{2}+q_{3} \omega_{3} \\ q_{0} \omega_{1}+q_{2} \omega_{3}-q_{3} \omega_{2} \\ q_{0} \omega_{2}+q_{3} \omega_{1}-q_{1} \omega_{3} \\ q_{0} \omega_{3}+q_{1} \omega_{2}-q_{2} \omega_{1}\end{array}\right]+K_{\varepsilon} \varepsilon\left[\begin{array}{c}q_{0} \\ q_{1} \\ q_{2} \\ q_{3}\end{array}\right]$,

donde $K_{\varepsilon}$ es una constante llamada ganancia de normalización.

El sistema (109) es siempre estable para $K_{\varepsilon}>0$. La demostración de esta aseveración se basa en que es posible comprobar que existe una función de Lyapunov que acota las funciones componentes del sistema. Por otro lado es posible encontrar que el límite de la región estable de (109) está dado por:

$h K_{\varepsilon} \leq 1$,

donde $h$ es el paso de la integración numérica.

Esto se demuestra fácilmente si multiplicamos a cada lado de la igualdad la primer fila de (104) por $q_{0}$, la segunda por $q_{1}$, la tercera por $q_{2}$ y la cuarta por $q_{3}$, y a continuación sumamos estas igualdades; los términos que contienen velocidades angulares se cancelan mutuamente y por tanto tenemos que:

$q_{0} q_{0}{ }^{\prime}+q_{1} q_{1}{ }^{\prime}+q_{2} q_{2}{ }^{\prime}+q_{3} q_{3}{ }^{\prime}=K_{\varepsilon} \varepsilon\left(q_{0}{ }^{2}+q_{1}{ }^{2}+q_{2}{ }^{2}+q_{3}{ }^{2}\right)$.

Esta ecuación puede ser escrita, con (105) y (106), como: 
$-\frac{\varepsilon^{\prime}}{2}=K_{\varepsilon} \varepsilon(1-\varepsilon)$

por lo tanto:

$-\varepsilon^{\prime} \approx 2 K_{\varepsilon} \varepsilon$

Adicionalmente se puede expresar que:

$\varepsilon^{\prime}=\frac{(\varepsilon)_{t+h}-(\varepsilon)_{t}}{h}=\frac{\Delta \varepsilon}{h}$

Por tanto:

$-\Delta \varepsilon \approx 2 K_{\varepsilon} h \varepsilon$

Para que el sistema sea estable es necesario que $|\varepsilon|$ sea decreciente para un número infinito de pasos de integración, por tanto:

$\left|(\varepsilon)_{t+h}\right| \leq\left|(\varepsilon)_{t}\right|$

y entonces:

$|\Delta \varepsilon|=\left|(\varepsilon)_{t+h}-(\varepsilon)_{t}\right| \leq\left|(\varepsilon)_{t+h}\right|-\left|(\varepsilon)_{t}\right| \leq 2\left|(\varepsilon)_{t}\right|$

Con (115) resulta por tanto:

$2|\varepsilon| \geq 2 K_{\varepsilon} h|\varepsilon|$

$h K_{\varepsilon} \leq 1$

Como los pasos de integración que se adopten serán siempre menores a 1/10 de segundo se adoptará un valor de $K_{\varepsilon}=1$, que cumplirá con la condición (119). 
Para estudiar la influencia del parámetro $K_{\varepsilon}$ en la solución, se considera un cuerpo rígido, con las condiciones iniciales que se muestran en la Tabla 3.1, y se simula su comportamiento dinámico durante 1000 segundos, basado en el esquema que se desarrolla en el presente capítulo, obteniéndose distintos cuaterniones de orientación final. Se adopta para el cálculo numérico un paso de integración fijo, de 0,005 segundos, utilizando el método de Dormand \& Prince (1980).

Tabla 0.1 Parámetros generales del ejemplo numérico

\begin{tabular}{|c|cl|}
\hline \multicolumn{3}{|c|}{ Parámetros generales } \\
\hline$I_{1}$ & $6,31 \cdot 10^{-4}$ & $\mathrm{~kg} \mathrm{~m}^{2}$ \\
\hline$I_{2}$ & $6,31 \cdot 10^{-4}$ & $\mathrm{~kg} \mathrm{~m}^{2}$ \\
\hline$I_{3}$ & $22,25 \cdot 10^{-4}$ & $\mathrm{~kg} \mathrm{~m}^{2}$ \\
\hline$\omega_{\text {inicial } 1}$ & 1 & $1 / \mathrm{s}$ \\
\hline$\omega_{\text {inicial } 2}$ & 0 & $1 / \mathrm{s}$ \\
\hline$\omega_{\text {inicial } 3}$ & 5 & $1 / \mathrm{s}$ \\
\hline
\end{tabular}

En la Tabla 3.2 se puede ver la influencia de la ganancia de normalización en el error por distorsión del cuaternión de orientación para el ejemplo numérico presentado. Se puede observar que cuando esta ganancia es cero, la distorsión numérica es relevante. Esta distorsión deriva en una matriz de transformación de coordenadas no ortonormal.

Tabla 0.2 Ganancia de ortonormalización y error para el cuaternión de orientación para la simulación del ejemplo numérico presentado.

\begin{tabular}{|c|c|}
\hline $\boldsymbol{K}_{\boldsymbol{\varepsilon}}$ & $\boldsymbol{\varepsilon}$ \\
\hline 0,00 & $1,9772 \cdot 10^{-3}$ \\
\hline
\end{tabular}




\begin{tabular}{|c|c|}
\hline $\boldsymbol{K}_{\boldsymbol{\varepsilon}}$ & $\boldsymbol{\varepsilon}$ \\
\hline 0,01 & $8,217 \cdot 10^{-5}$ \\
\hline 0,10 & $7,837 \cdot 10^{-6}$ \\
\hline 0,50 & $1,323 \cdot 10^{-6}$ \\
\hline 1,00 & $4,441 \cdot 10^{-7}$ \\
\hline 10,00 & $1,587 \cdot 10^{-6}$ \\
\hline
\end{tabular}

\subsubsection{Condiciones iniciales y de salida}

Las condiciones de orientación iniciales para el comienzo de la simulación corresponden a la definición del conjunto de ángulos de Euler que la describan:

$$
\operatorname{Eul}_{0}=\left(\varphi_{0}, \theta_{0}, \psi_{0}\right)
$$

La orientación inicial se transformará en un cuaternión inicial según la siguiente expresión:

$$
\mathbf{q}(\varphi, \theta, \psi)=\frac{1}{2}\left[\begin{array}{c}
\cos \frac{\varphi}{2} \cos \frac{\theta}{2} \cos \frac{\psi}{2}-\sin \frac{\varphi}{2} \cos \frac{\theta}{2} \sin \frac{\psi}{2} \\
\cos \frac{\varphi}{2} \sin \frac{\theta}{2} \cos \frac{\psi}{2}+\sin \frac{\varphi}{2} \sin \frac{\theta}{2} \sin \frac{\psi}{2} \\
\cos \frac{\varphi}{2} \sin \frac{\theta}{2} \sin \frac{\psi}{2}-\sin \frac{\varphi}{2} \sin \frac{\theta}{2} \cos \frac{\psi}{2} \\
\cos \frac{\varphi}{2} \cos \frac{\theta}{2} \sin \frac{\psi}{2}+\cos \frac{\varphi}{2} \sin \frac{\theta}{2} \cos \frac{\psi}{2}
\end{array}\right] .
$$

Por otro lado el resultado de salida de la integración de la expresión (119) será un cuaternión de orientación que será la condición inicial del intervalo de integración posterior. Sucesivamente, entonces, se reinyectará el resultado del cómputo de (119) para obtener la simulación de la actitud del pararrotor. El resultado del cómputo de (119) se usa para establecer las condiciones iniciales del paso de integración posterior, y representa también la salida del paso de integración en cuestión. 
El mencionado cuaternión de salida se transforma en un conjunto de ángulos de Euler (de ordenamiento 313 , que se corresponde con rotaciones de Euler, primero sobre el eje 3, luego sobre el 1 y finalmente sobre el 3, equivalente a las rotaciones de precesión, nutación, y rotación propia) para facilitar su interpretación física, a partir de las siguientes expresiones:

$$
\begin{aligned}
& \varphi=\operatorname{atan}\left(\frac{q_{1} q_{3}-q_{0} q_{2}}{q_{2} q_{3}+q_{0} q_{1}}\right), \\
& \theta=\operatorname{acos}\left(q_{0}^{2}-q_{1}^{2}-q_{2}^{2}+q_{3}^{2}\right), \\
& \psi=\operatorname{atan}\left(\frac{q_{1} q_{3}+q_{0} q_{2}}{q_{0} q_{1}-q_{2} q_{3}}\right) .
\end{aligned}
$$

Así mismo es posible obtener del cuaternión de salida una matriz de rotación (o matriz de cosenos directores que se denominará DCM) para la transformación de vectores entre sistemas coordenados a partir de la siguiente expresión:

$$
\operatorname{DCM}(\mathbf{q})=\left[\begin{array}{ccc}
q_{0}{ }^{2}+q_{1}{ }^{2}-q_{2}{ }^{2}-q_{3}{ }^{2} & 2 q_{1} q_{2}+2 q_{0} q_{3} & 2 q_{1} q_{3}-2 q_{0} q_{2} \\
2 q_{1} q_{2}-2 q_{0} q_{3} & q_{0}^{2}-q_{1}^{2}+q_{2}^{2}-q_{3}^{2} & 2 q_{2} q_{3}+2 q_{0} q_{1} \\
2 q_{1} q_{3}+2 q_{0} q_{2} & 2 q_{2} q_{3}-2 q_{0} q_{1} & q_{0}^{2}-q_{1}^{2}-q_{2}^{2}+q_{3}^{2}
\end{array}\right]
$$

Si se considera un vector $\mathbf{z}$ genérico en la terna coordenada inercial y el mismo vector pero expresado en la terna coordenada móvil, adherida al pararrotor, con denominación $z_{c}$, tendremos que:

$$
\begin{aligned}
& \mathbf{z}_{\mathbf{c}}=\operatorname{DCM}(\mathbf{q}) \mathbf{z} \\
& \mathbf{z}=\operatorname{DCM}(\mathbf{q})^{\mathrm{T}} \mathbf{z}_{\mathbf{c}}
\end{aligned}
$$

\subsubsection{Modelo gráfico}

El modelo gráfico que representa el proceso de integración numérica, asociado a la expresión (109), que se explicita en este apartado, se presenta en la Figura 3.2. 
Se define al vector $\mathbf{u}$ de la siguiente manera:

$\mathbf{u}=\left(\omega_{1}, \omega_{2}, \omega_{3}, q_{0}, q_{1}, q_{2}, q_{3}, \varepsilon\right)$

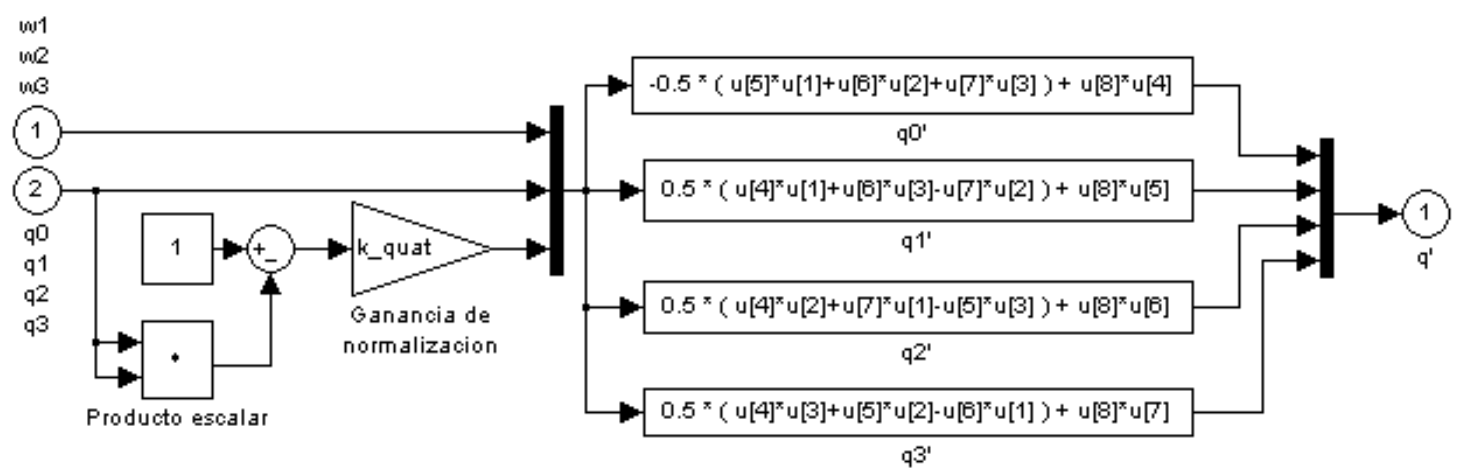

Figura 0.2 Modelo gráfico del cálculo de la derivada temporal del cuaternión de orientación

A los efectos de la representación este modelo gráfico se sintetiza en un bloque único que se denominará "qdot".

El modelo gráfico que formaliza los cómputos de la orientación se presenta en la Figura 3.3. 


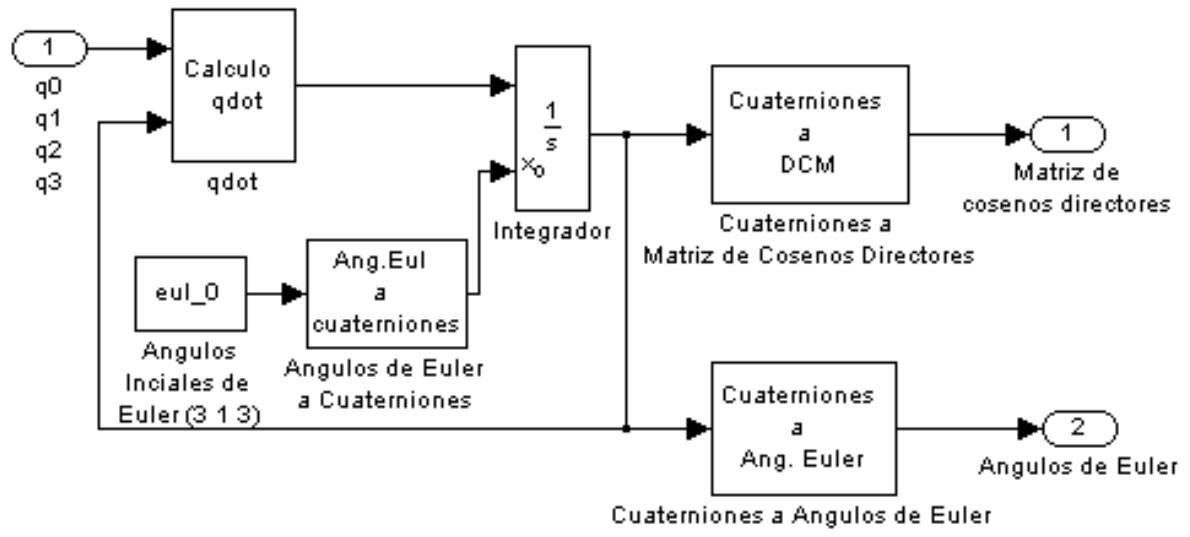

Figura 0.3 Modelo gráfico del cómputo de la orientación del pararrotor.

La conversión de cuaterniones a la matriz de cosenos directores tiene la siguiente representación gráfica asociada con (125), que se muestra en la Figura 3.4. 


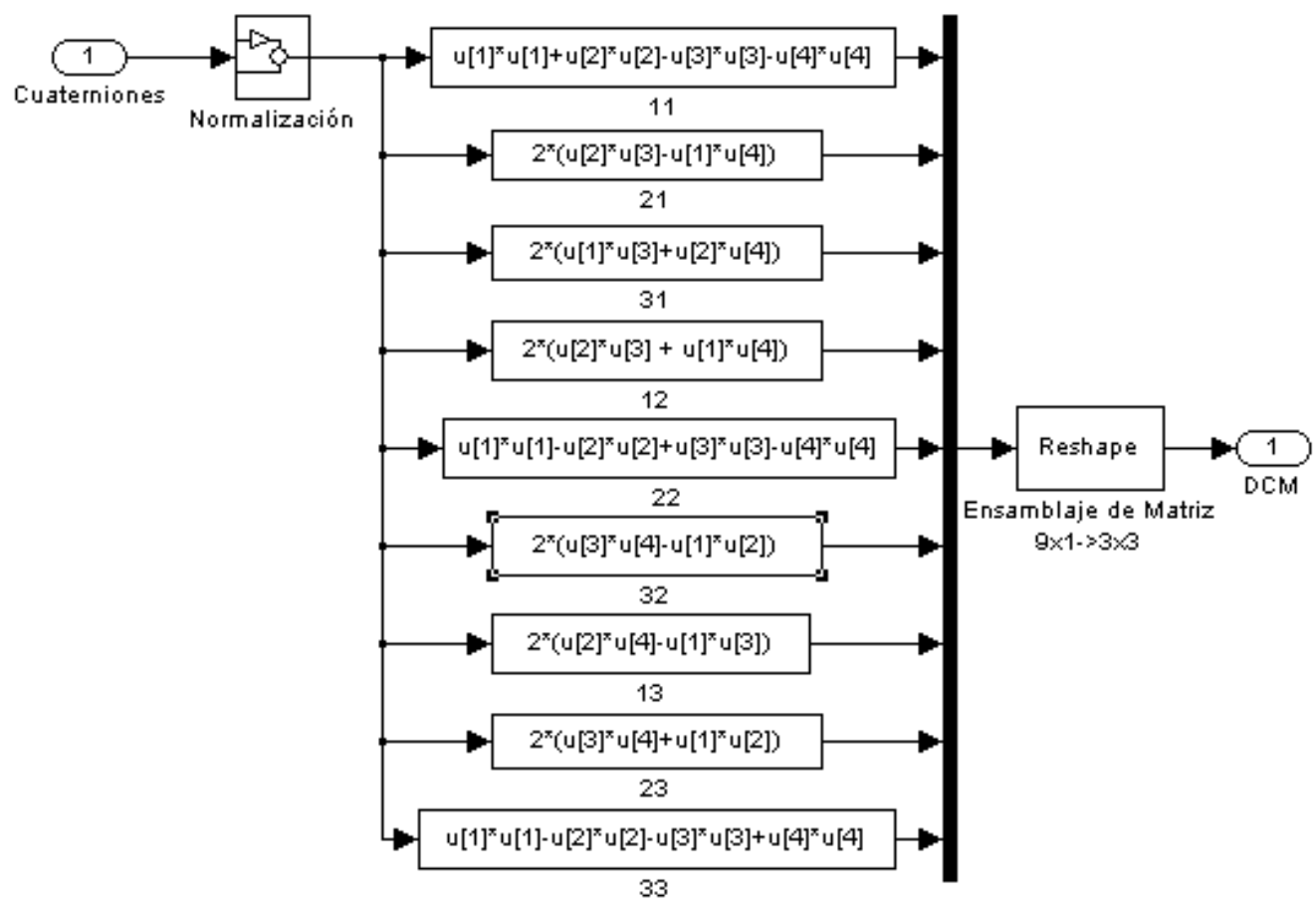

Figura 0.4 Modelo gráfico del cómputo de la conversión de cuaterniones a la Matriz de Cosenos Directores (denominada DCM).

La conversión de cuaterniones a ángulos de Euler (con orden de orientación 3113 ), que sintéticamente tiene la siguiente representación gráfica asociada a (122) (123) (124), que se muestra en la Figura 3.5, donde $\mathbf{u}$, en este caso, representa el vector dado por las componentes del cuaternión ortonormalizado de orientación. 


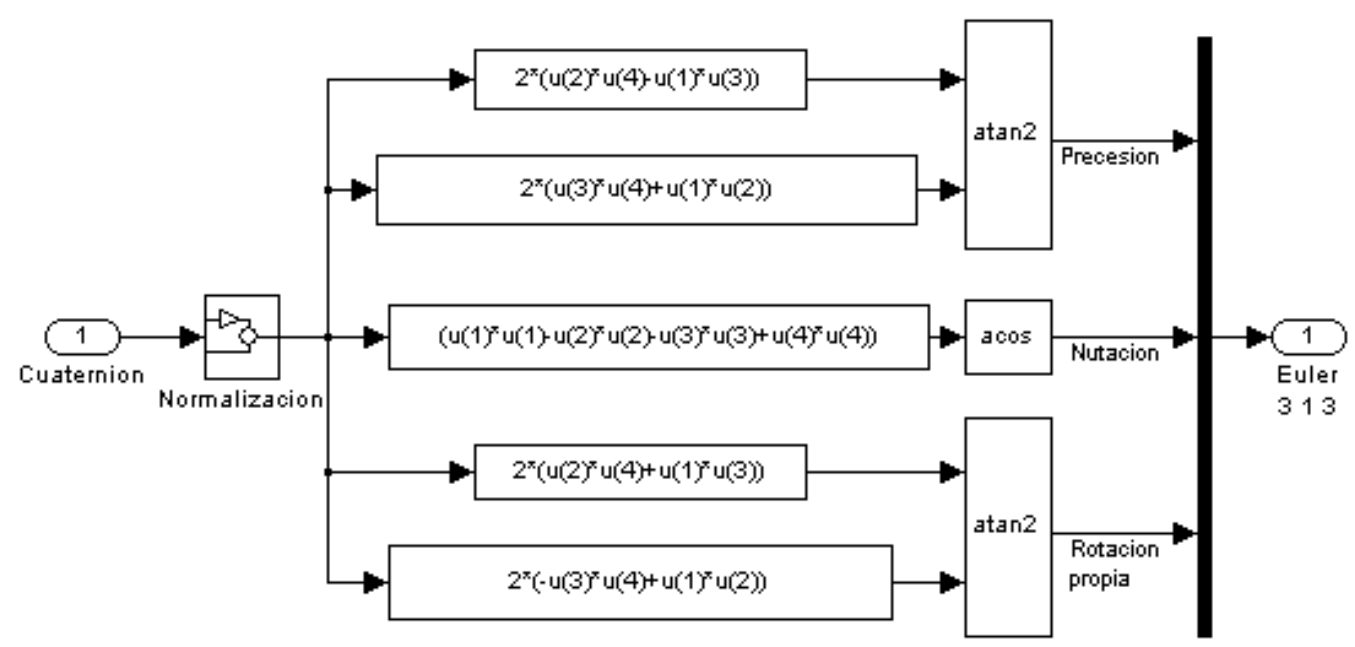

Figura 0.5 Modelo gráfico del cómputo de la conversión de cuaterniones a ángulos de Euler (con orden de rotación 313 ).

\subsubsection{Ecuaciones de la dinámica del pararrotor}

Las ecuaciones de la dinámica del pararrotor, de 6 grados de libertad, resultan de considerar la dinámica de rotación y translación, las cuales se encuentran acopladas.

Las potencialidades de este modelo residen en poder simular situaciones cercanas a la realidad física del vuelo del pararrotor. Las expresiones de las ecuaciones de la dinámica son:

$$
\begin{aligned}
& \frac{M_{1}}{I_{1}}=\frac{\mathrm{d} \omega_{1}}{\mathrm{~d} t}-\left(\frac{I_{2}-I_{3}}{I_{1}}\right) \omega_{3} \omega_{2} \\
& \frac{M_{2}}{I_{2}}=\frac{\mathrm{d} \omega_{2}}{\mathrm{~d} t}+\left(\frac{I_{1}-I_{3}}{I_{2}}\right) \omega_{3} \omega_{1} \\
& \frac{M_{3}}{I_{3}}=\frac{\mathrm{d} \omega_{3}}{\mathrm{~d} t}-\left(\frac{I_{1}-I_{2}}{I_{3}}\right) \omega_{2} \omega_{1} \\
& \frac{F_{1}}{m}=\frac{\mathrm{d} x_{1}}{\mathrm{~d} t}+\omega_{3} V_{b 2}-\omega_{2} V_{b 3}
\end{aligned}
$$




$$
\begin{aligned}
& \frac{F_{2}}{m}=\frac{\mathrm{d} x_{2}}{\mathrm{~d} t}+\omega_{1} V_{b 3}-\omega_{3} V_{b 1} \\
& \frac{F_{3}}{m}=\frac{\mathrm{d} x_{3}}{\mathrm{~d} t}+\omega_{2} V_{b 1}-\omega_{1} V_{b 2}
\end{aligned}
$$

La velocidad de caída en coordenadas del cuerpo será el producto de la matriz de rotación asociada a la transformación de Euler adoptada y la velocidad relativa de los ejes cuerpo al respecto de los inerciales.

\subsubsection{Modelo gráfico}

La estrategia de cómputo numérico de las ecuaciones diferenciales de la dinámica se basan en la integración en pasos temporales, a partir de valores iniciales, de:

- Aceleración angular

- Aceleración translacional

- Velocidad traslacional

Se realizarán entonces tres procesos de integración sobre cada uno de estos parámetros, que permitirán conocer la evolución de la dinámica del pararrotor sobre la base de datos de iniciales. El resultado de estos procesos entregan resultados sobre:

- Velocidad angular

- Velocidad translacional

- Posición

El modelo gráfico de esta formulación se muestra en la Figura 3.6, incluyendo el modelo de cómputo de la orientación y parámetros de salida referidos a la terna coordenada inercial. 


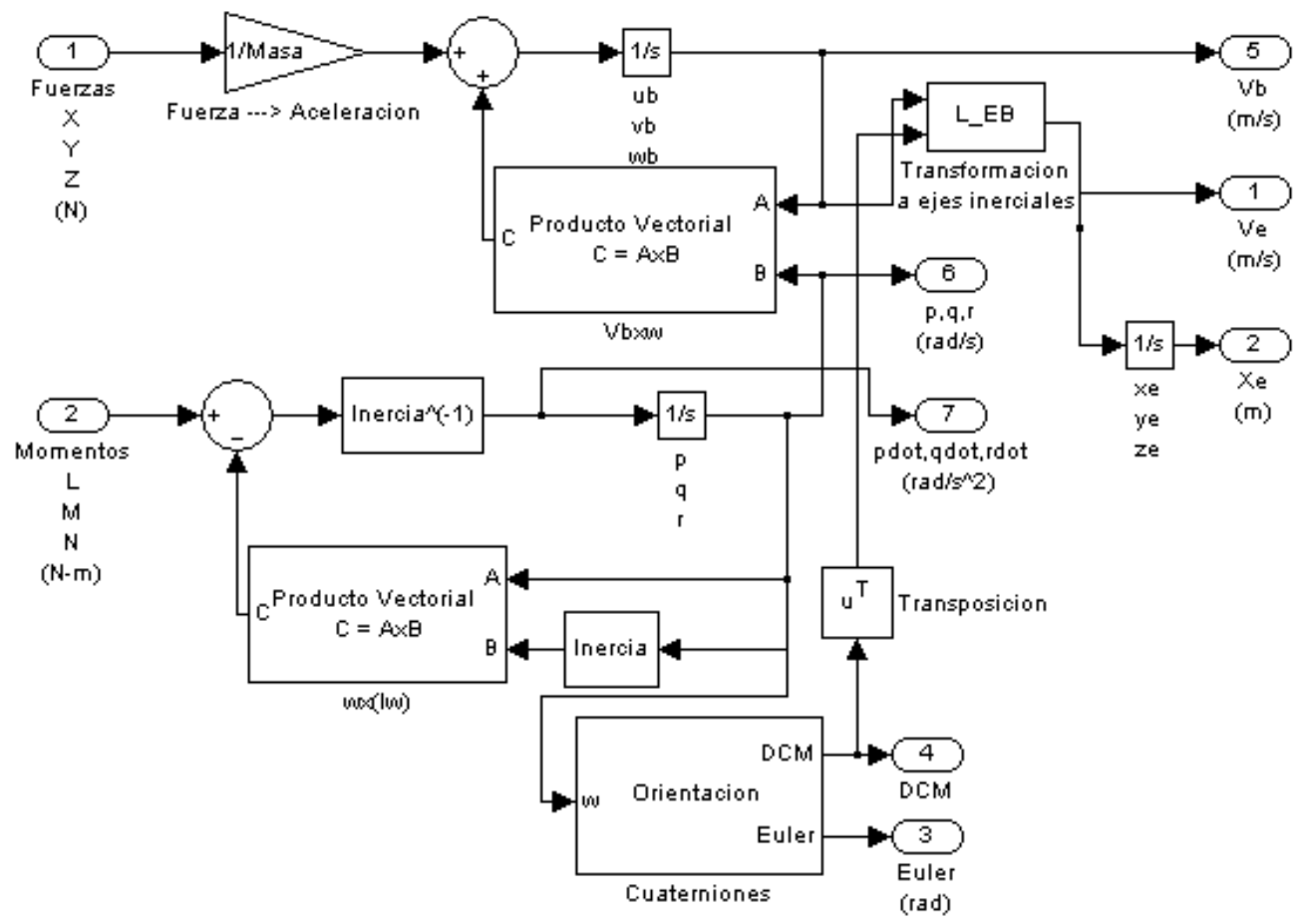

Figura 0.6 Modelo gráfico del cómputo de la dinámica y orientación del pararrotor. $\mathrm{V}_{\boldsymbol{e}} \mathrm{y}$ $\mathrm{X}_{e}$ son la velocidad y posición en coordenadas inerciales.

El modelo gráfico de la Figura 3.7 muestra las integrales bajo la denominación " $1 / \mathrm{s}$ ”, que proviene de la nomenclatura empleada en la transformada de Laplace. Los datos de entrada, independientemente de las fuerzas y los momentos, que permiten la ejecución de esta rutina de cálculo, incluyendo los cálculos asociados a la determinación de la orientación del pararrotor en el tiempo, son entonces:

- Parámetros de simulación:

o Tiempo de integración

o Método de integración numérica 
o Tipo de paso de integración (fijo o variable)

- Velocidad inicial - Ejes inerciales

- Orientación inicial - Euler - Ejes inerciales

- Velocidad angular inicial - Ejes cuerpo

\subsubsection{Velocidades relativas}

Se considera la velocidad $\mathbf{V}_{t c}$ correspondiente a la velocidad del flujo incidente en el pararrotor con respecto a los ejes inerciales expresado en coordenadas del cuerpo, adoptando como hipótesis que la atmósfera se encuentra en reposo. Esta velocidad es idéntica pero de signo contrario a la velocidad del pararrotor con respecto al sistema de coordenadas inercial, bajo la hipótesis de atmósfera en reposo.

Es menester mencionar que la velocidad preponderante de este vector es aquella correspondiente a la velocidad de descenso, que tiene la dirección del eje $Z$ inercial (que se puede denominar como eje vertical).

La velocidad del fluido relativa a la pala 1 será:

$$
\mathbf{V}_{r 1}=-\left[\begin{array}{c}
\omega_{1} \\
\omega_{2} \\
\omega_{3}
\end{array}\right] \times\left[\begin{array}{l}
r_{11} \\
r_{12} \\
r_{13}
\end{array}\right]+\mathbf{V}_{t c}
$$

Para la pala 2 se tiene que:

$$
\mathbf{V}_{r 2}=-\left[\begin{array}{c}
\omega_{1} \\
\omega_{2} \\
\omega_{3}
\end{array}\right] \times\left[\begin{array}{c}
-r_{11} \\
-r_{12} \\
r_{13}
\end{array}\right]+\mathbf{V}_{t c}
$$

Se muestra en la Figura 3.7 un esquema de los componentes de la velocidad relativa para la pala 1. En esta misma figura se muestra la configuración geométrica del pararrotor con la 
nomenclatura adoptada para denominar la ubicación del Centro de Masas y el Centro de Presiones.

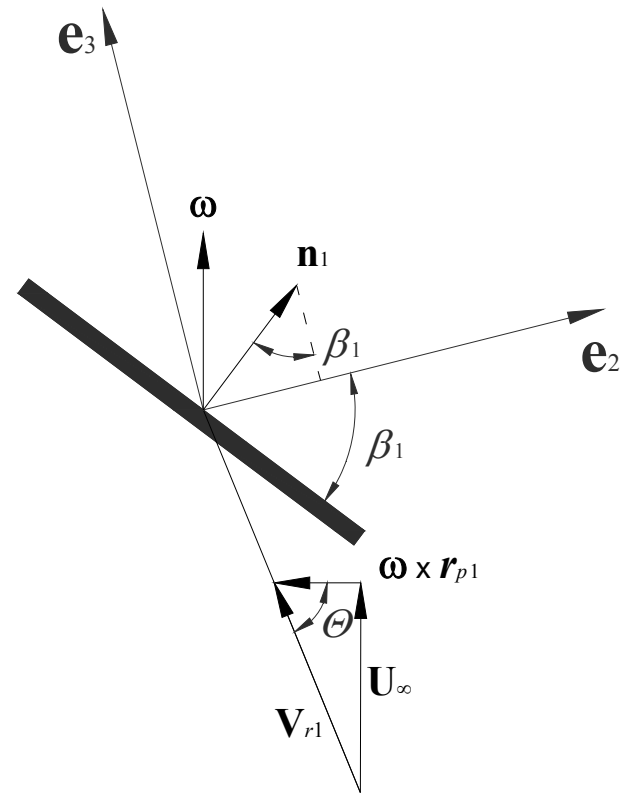

Figura 0.7 Velocidad relativa a la pala 1 en componentes sobre el plano $e_{2}-e_{3}$. 

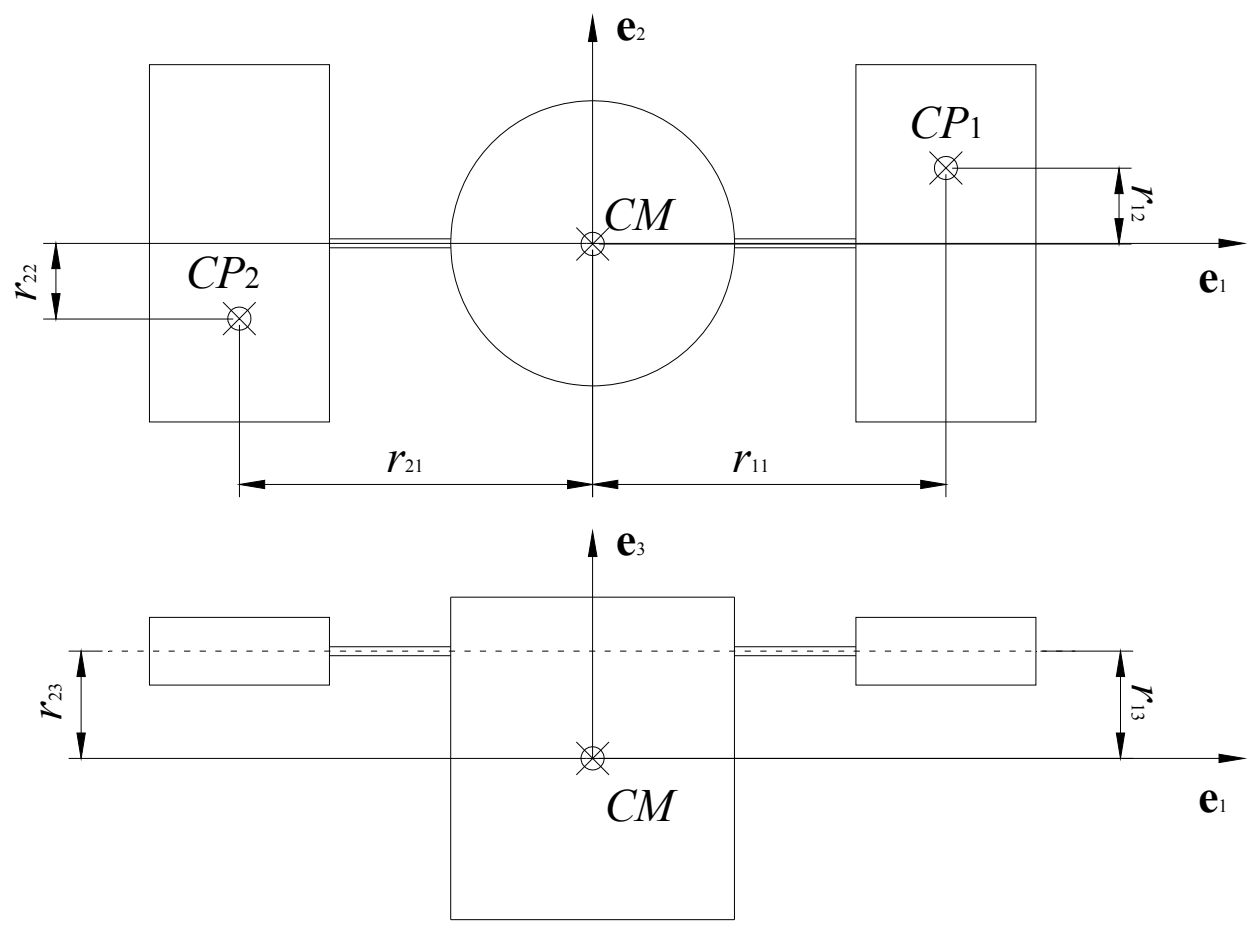

Figura 0.8 Posición del centro de presiones de las palas al respecto del centro de masas.

\subsubsection{Modelo gráfico}

Los modelos gráficos para el cálculo numérico, que en adelante estará representado por los bloques Velocidad Relativa - Pala 1 y Velocidad Relativa - Pala 2 se muestran en la Figura 3.9. y la Figura 3.10. 


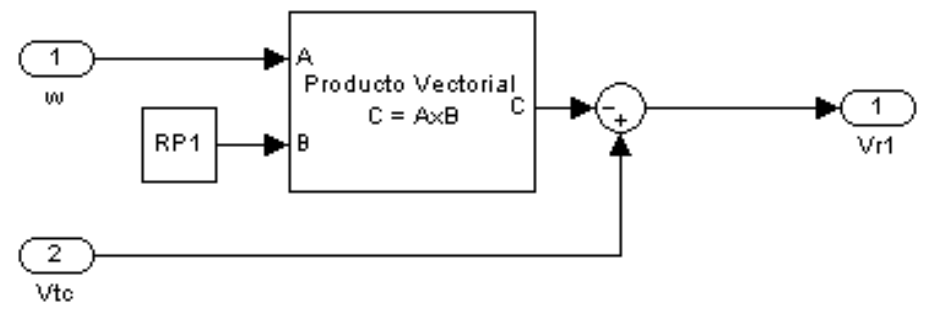

Figura 0.9 Modelo gráfico de la determinación de la velocidad relativa a la pala 1.

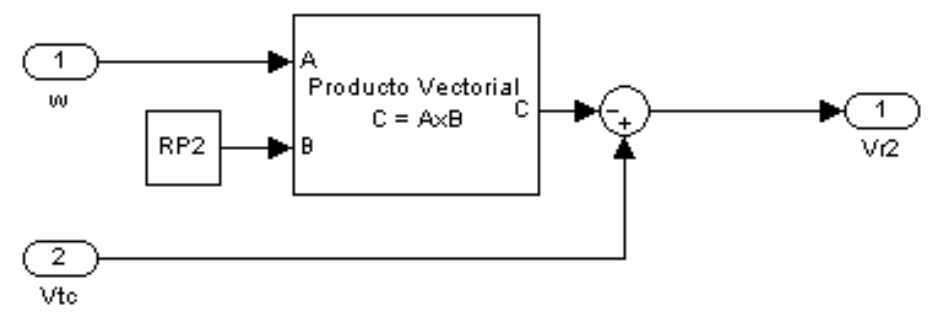

Figura 0.10 Modelo gráfico de la determinación de la velocidad relativa a la pala 2.

\subsubsection{Versores}

Se consideran una serie de vectores normalizados en las direcciones de la sustentación y de la resistencia (Figura 3.11), para, a posteriori, asignarles las magnitudes de las fuerzas aerodinámicas. 


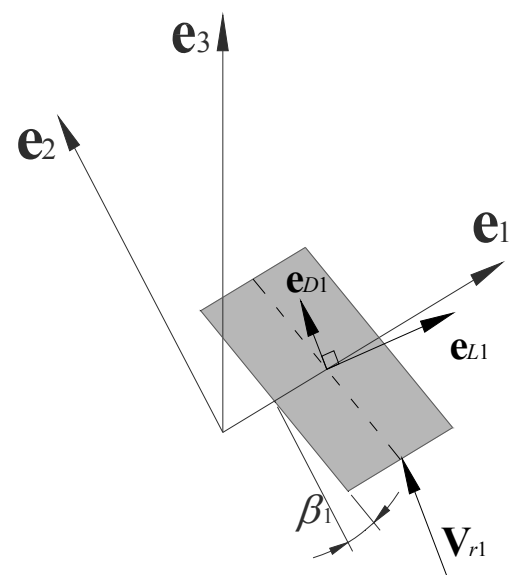

Figura 0.11 Versores asociados a la pala 1.

El vector sustentación será normal al plano formado por la velocidad relativa y el eje según la envergadura de la pala que se esté considerando (que tendrá la dirección del eje 1). Del vector resultante no se considerará la componente en dirección $\mathbf{e}_{1}$.Vale decir que estos versores serán:

$\mathbf{e}_{L 1}=\frac{\left(\left[\begin{array}{c}-1 \\ 0 \\ 0\end{array}\right] \times \mathbf{V}_{r 1}\right)}{\left|\left(\left[\begin{array}{c}-1 \\ 0 \\ 0\end{array}\right] \times \mathbf{V}_{r 1}\right)\right|}$

$\mathbf{e}_{L 2}=\frac{\left(\left[\begin{array}{l}1 \\ 0 \\ 0\end{array}\right] \times \mathbf{V}_{r 2}\right)}{\left|\left(\left[\begin{array}{l}1 \\ 0 \\ 0\end{array}\right] \times \mathbf{V}_{r 2}\right)\right|}$ 
Los versores resistencia tendrán la dirección de la velocidad relativa a cada pala, exceptuando los componentes en dirección al eje $\mathbf{e}_{1}$ :

$\mathbf{e}_{D 1}=\frac{\mathbf{V}_{r 1}}{\left|\mathbf{V}_{r 1}\right|}$

$\mathbf{e}_{D 2}=\frac{\mathbf{V}_{r 2}}{\left|\mathbf{V}_{r 2}\right|}$

\subsubsection{Modelo gráfico}

Los modelos gráficos para el cálculo numérico, que en adelante estarán representados por los bloques Versor Sustentación - Pala 1 y Versor Sustentación - Pala 2 se muestran en las Figuras 3.12 y 3.13 .

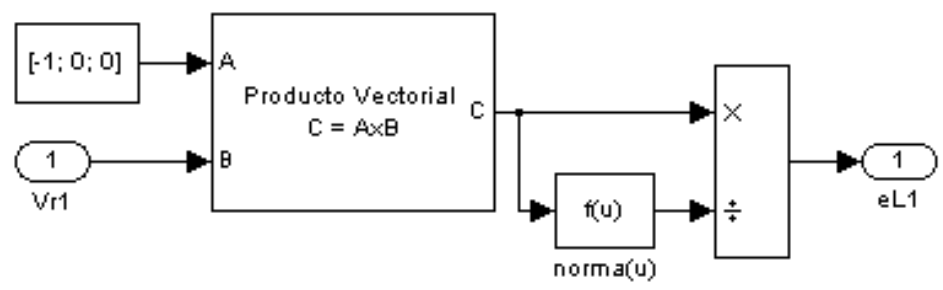

Figura 0.12 Versor sustentación asociado a la pala 1.

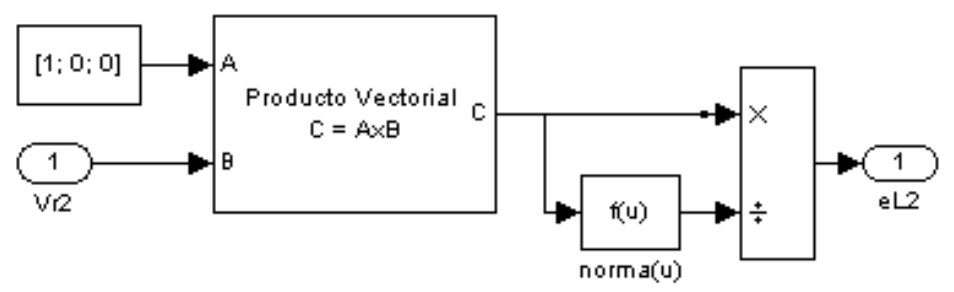

Figura 0.13 Versor sustentación asociado a la pala 2. 
Los modelos gráficos para el cálculo numérico, que en adelante estarán representados por los bloques Versor Resistencia - Pala 1 y Versor Resistencia - Pala 2 se muestran en las Figuras 3.14 y 3.15 .

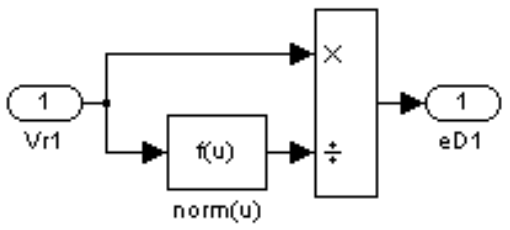

Figura 0.14 Versor resistencia asociado a la pala 1.

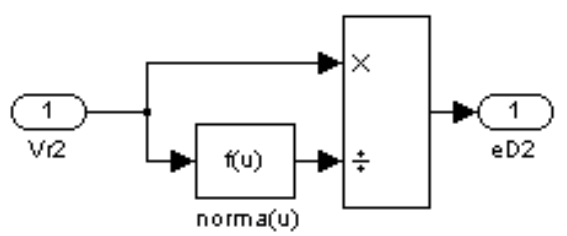

Figura 0.15 Versor resistencia asociado a la pala 2.

\subsection{6 Ángulos de ataque}

Los ángulos de ataque (Figura 3.16) para las palas $i=1,2$ serán:

$\sin \alpha_{1}=\frac{\mathbf{n}_{1} \mathbf{V}_{r 1}}{\left|\mathbf{V}_{r 1}\right|}$ 


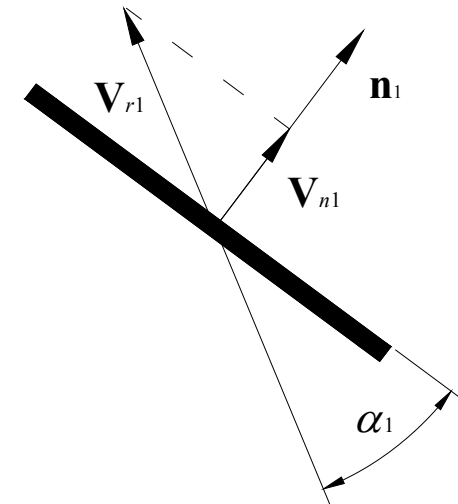

Figura 0.16 Determinación del ángulo de ataque para la pala 1.

Los vectores unitarios normales a cada plano de las palas responderán a las siguientes expresiones:

$\mathbf{n}_{1}=\left[\begin{array}{c}0 \\ \sin \beta_{1} \\ \cos \beta_{1}\end{array}\right]$

$\mathbf{n}_{2}=\left[\begin{array}{c}0 \\ -\sin \beta_{2} \\ \cos \beta_{2}\end{array}\right]$

\subsubsection{Modelo gráfico}

Los modelos gráficos para el cálculo numérico, que en adelante estarán representados por los bloques Angulo de Ataque - Pala 1 y Angulo de Ataque - Pala 2 se muestran en las Figuras 3.17 y 3.18 . 


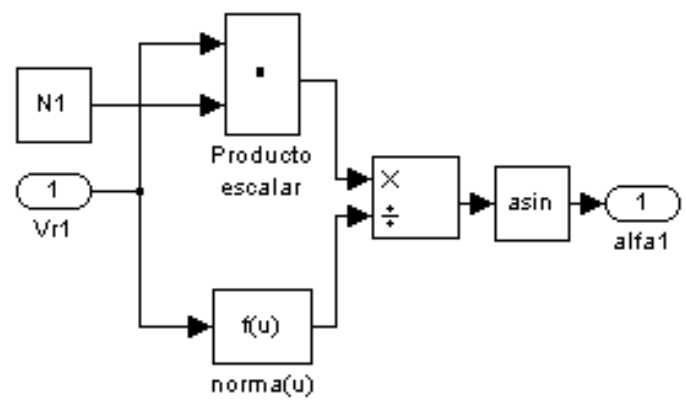

Figura 0.17 Modelo gráfico del cálculo del ángulo de ataque para la pala 1.

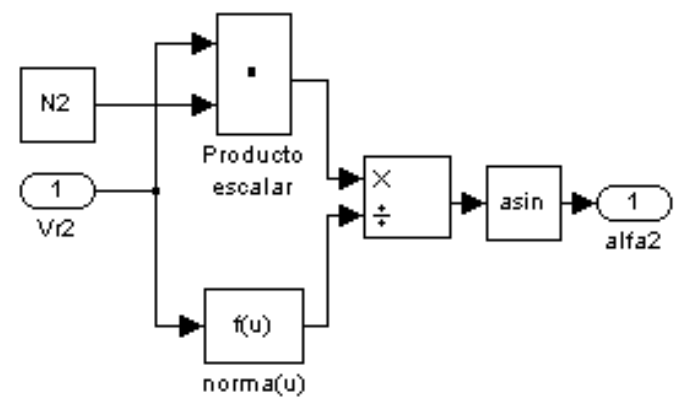

Figura 0.18 Modelo gráfico del cálculo del ángulo de ataque para la pala 2.

\subsubsection{Fuerzas aerodinámicas}

La determinación de las fuerzas y momentos generados en las palas del pararrotor son la base de la simulación de su comportamiento dinámico.

Se considerará como simplificación que en el transcurso del movimiento los ejes cuerpo se mantendrán cercanos a una situación de equilibrio estable, donde el eje 3 del cuerpo se encuentra próximo a la vertical inercial (eje Z).

Las fuerzas de sustentación y resistencia se obtienen a partir de las expresiones (144) y (145), que consideran que la componente de la velocidad relativa en dirección a $\mathbf{e}_{1}$ no genera fuerzas aerodinámicas asociadas al perfil aerodinámico de las palas del pararrotor, para $i=1,2$ : 


$$
\begin{aligned}
& \mathbf{L}_{i}=\frac{1}{2} \rho S C_{L \alpha} \alpha_{i}\left|\mathbf{V}_{r i}\right|^{2} \mathbf{e}_{L i}, \\
& \mathbf{L}_{\mathrm{i}}=\frac{1}{2} \rho S C_{D}\left|\mathbf{V}_{r i}\right|^{2} \mathbf{e}_{D i} .
\end{aligned}
$$

\subsubsection{Modelo gráfico}

Los modelos gráficos que sintetizarán el procedimiento de cálculo se muestran en las figuras a continuación. Las Figuras 3.19 y 3.20 muestran el modelo gráfico del cómputo de las fuerzas aerodinámicas de sustentación para la pala 1 y la pala 2 respectivamente. Las Figuras 3.21 y 3.22 muestran el modelo gráfico del cómputo de las fuerzas aerodinámicas de resistencia para la pala 1 y la pala 2 respectivamente. La Figura 3.23 muestra el modelo gráfico del cómputo de las fuerzas aerodinámicas en general, partiendo del cómputo de las velocidades relativas cuya información de entrada está dada por la velocidad rotacional y la velocidad traslacional del cuerpo.

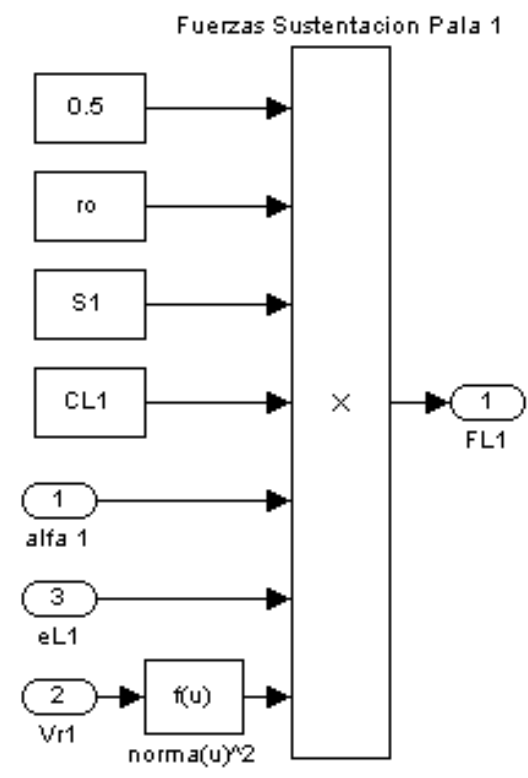

Figura 0.19 Modelo gráfico del cálculo de la fuerza de sustentación para la pala 1. 


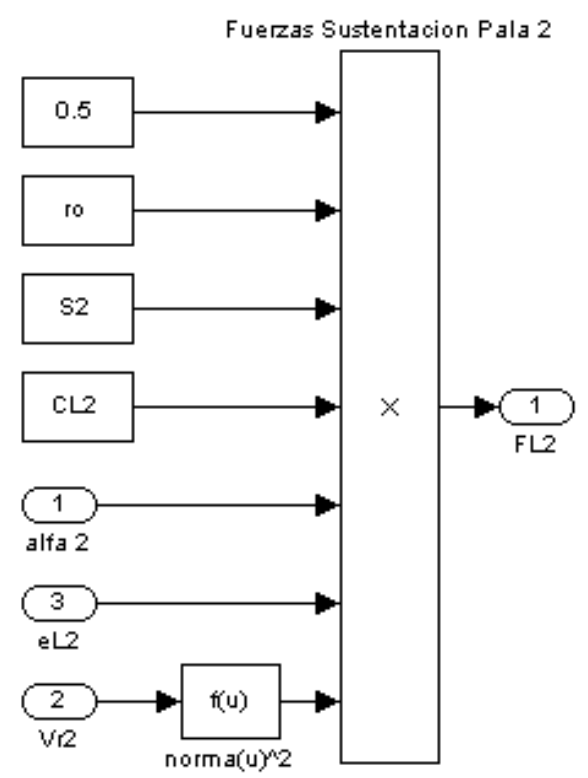

Figura 0.20 Modelo gráfico del cálculo de la fuerza de sustentación para la pala 2.

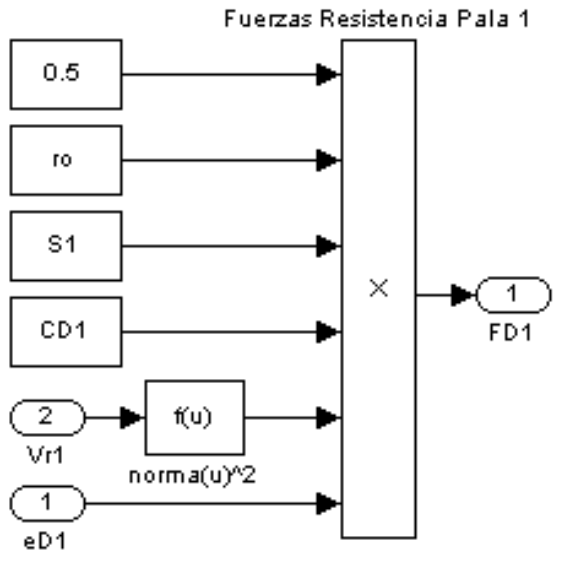

Figura 0.21 Modelo gráfico del cálculo de la resistencia para la pala 1. 


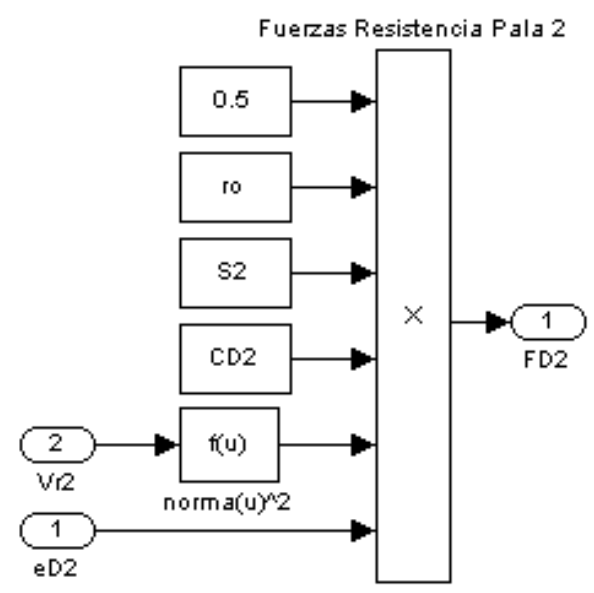

Figura 0.22 Modelo gráfico del cálculo de la resistencia para la pala 2. 


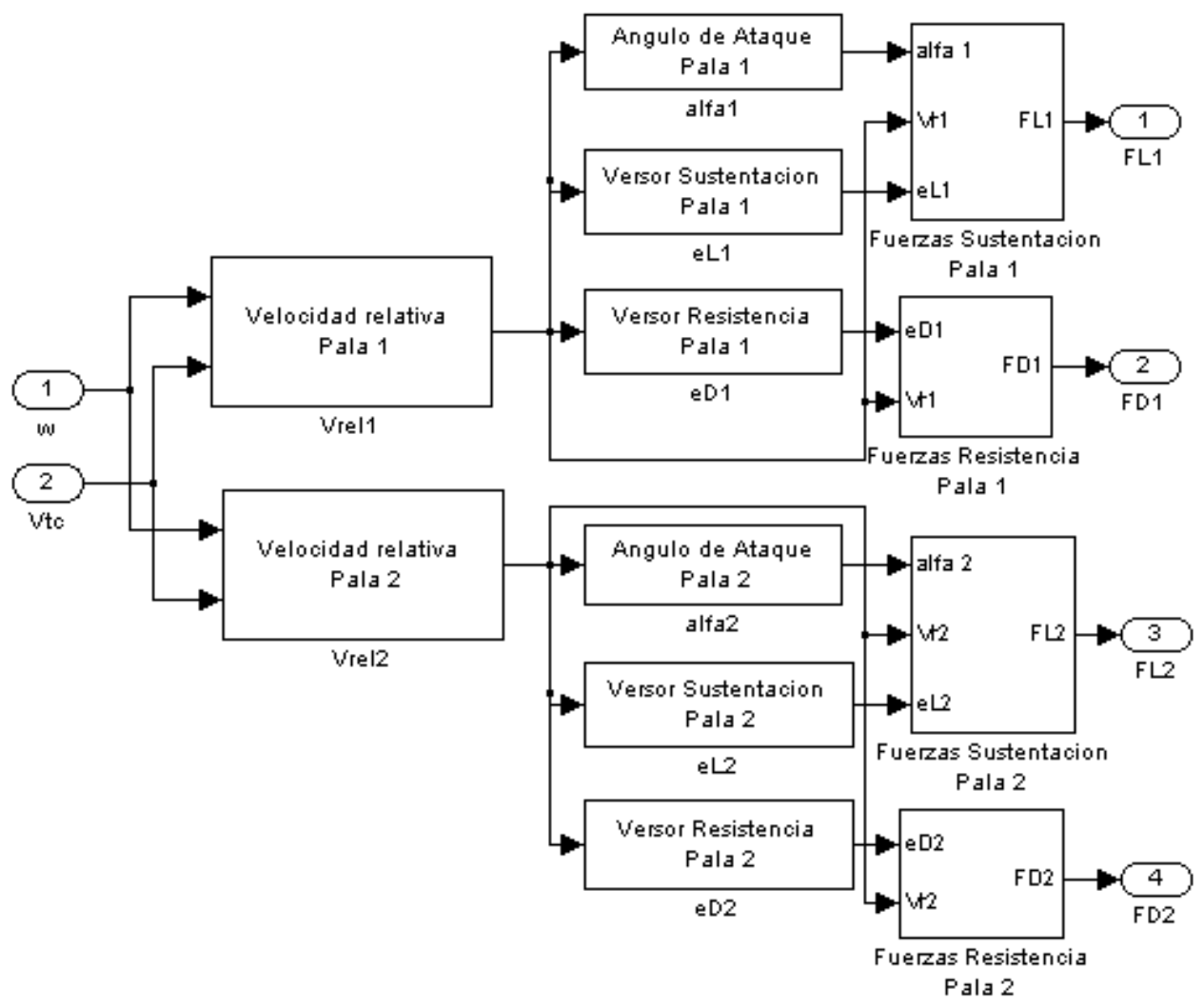

Figura 0.23 Modelo gráfico general del cálculo de las fuerzas aerodinámicas de las palas.

\subsubsection{Fuerza de la gravedad}

La gravedad representa la fuente de energía de la dinámica del pararrotor, por cuanto este se puede entender como un dispositivo que convierte energía potencial en cinética (disipando una parte por la acción de la resistencia aerodinámica). El cómputo de la fuerza gravitatoria depende del vector de aceleración gravitatoria local, en ternas inerciales, y de la masa del cuerpo. Para su consideración en el presente modelo de simulación resulta necesario transformar el vector inercial de aceleración gravitatoria al sistema móvil de ternas coordenadas adherido al pararrotor. La Matriz de Cosenos Directores, expresada en la ecuación (125), permite la transformación de vectores entre el sistema inercial de ternas coordenadas y el móvil adherido al pararrotor, como se muestra en las ecuaciones (126) y (127). 
El valor numérico de esta matriz será resultado del cómputo de la orientación del pararrotor en el tiempo. La expresión para la determinación de la fuerza de gravedad en coordenadas cuerpo resulta:

$$
\mathrm{F}_{g c}=\mathbf{D M C} m \mathbf{g}=\left[\begin{array}{lll}
D C M_{11} & D C M_{12} & D C M_{13} \\
D C M_{21} & D C M_{22} & D C M_{23} \\
D C M_{31} & D C M_{32} & D C M_{33}
\end{array}\right]\left[\begin{array}{c}
0 \\
0 \\
-m g
\end{array}\right]
$$

\subsubsection{Modelo gráfico}

El modelo gráfico de la expresión (146) se muestra en la Figura 3.24.

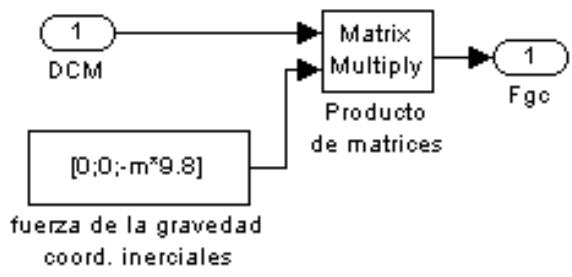

Figura 0.24 Modelo gráfico del cálculo de la fuerza de gravedad.

\subsubsection{Momentos respecto al centro de masas}

Los momentos generados al respecto de centro de masas son los generados por las fuerzas aerodinámicas de ambas palas. Por su parte la fuerza de gravedad se encuentra aplicada en el centro de masas. Se adopta por tanto la siguiente expresión:

$\mathbf{M}=\left[\left[\begin{array}{l}r_{11} \\ r_{12} \\ r_{13}\end{array}\right] \times\left[\begin{array}{c}F_{L 11} \\ F_{L 12} \\ F_{L 13}\end{array}\right]+\left[\begin{array}{l}r_{11} \\ r_{12} \\ r_{13}\end{array}\right] \times\left[\begin{array}{l}F_{D 11} \\ F_{D 12} \\ F_{D 13}\end{array}\right]+\left[\begin{array}{c}-r_{11} \\ -r_{12} \\ r_{13}\end{array}\right] \times\left[\begin{array}{c}F_{L 21} \\ F_{L 22} \\ F_{L 23}\end{array}\right]+\left[\begin{array}{c}-r_{11} \\ -r_{12} \\ r_{13}\end{array}\right] \times\left[\begin{array}{c}F_{D 11} \\ F_{D 12} \\ F_{D 23}\end{array}\right]\right]$.

\subsubsection{Modelo gráfico}

El modelo gráfico de la (147) se muestra en las Figuras 3.25 y 3.26. 


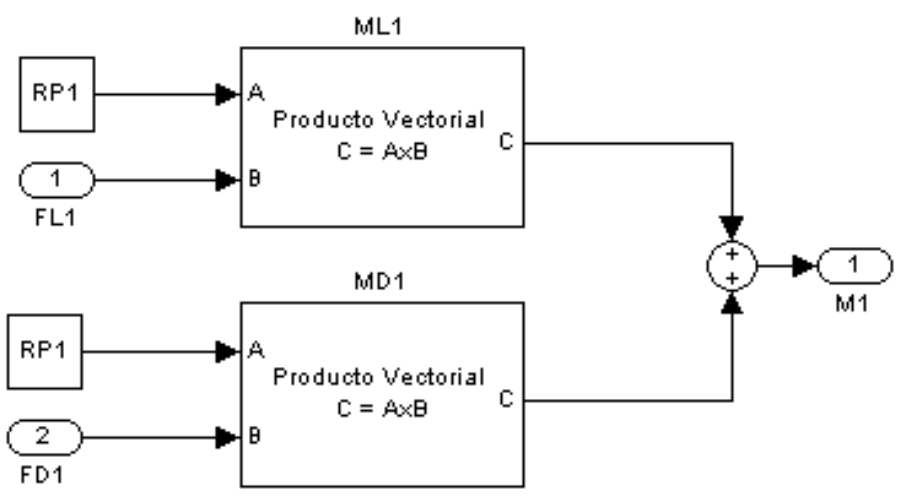

Figura 0.25 Modelo gráfico del cálculo de los momentos producidos por la pala 1.

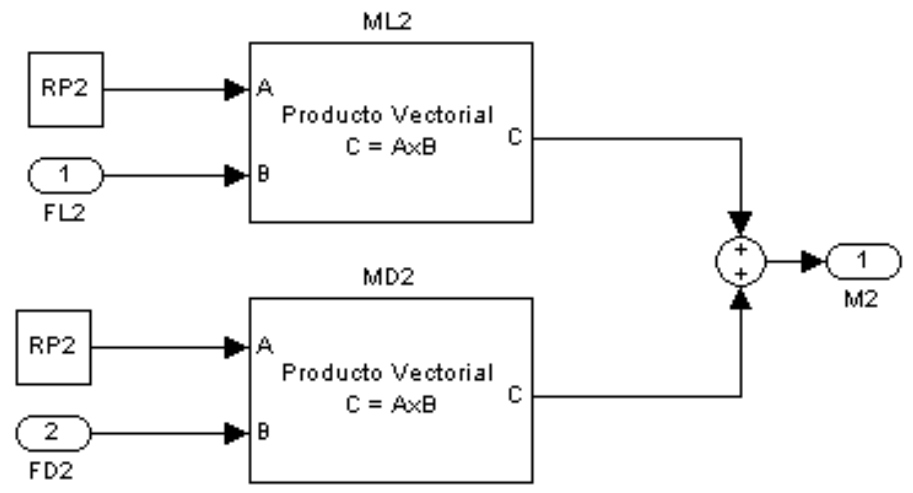

Figura 0.26 Modelo gráfico del cálculo de los momentos producidos por la pala 2.

\subsection{CASO NUMÉRICO}

A los fines de la comprobación del cálculo de fuerzas y momentos se presentará un caso cuya solución analítica es conocida, se adoptará una configuración específica, cuyos parámetros generales se muestran en la Tabla 3.3, y se cotejarán los resultados. 
Tabla 0.3 Constantes del caso de evaluación numérica.

\begin{tabular}{|c|c|c|}
\hline \multicolumn{3}{|c|}{ Parámetros generales } \\
\hline$C_{L \alpha}$ & 3,4 & {$[1 / \mathrm{rad}]$} \\
\hline$C_{D}$ & 0,07 & {$[-]$} \\
\hline$\rho$ & 1,21 & {$\left[\mathrm{~kg} / \mathrm{m}^{3}\right]$} \\
\hline$\omega_{0}$ & 379,3 & {$[\mathrm{rad} / \mathrm{s}]$} \\
\hline$S$ & 0,012 & {$\left[\mathrm{~m}^{2}\right]$} \\
\hline$r_{11}, r_{21}$ & 0,1 & {$[\mathrm{~m}]$} \\
\hline$r_{12}, r_{22}$ & 0,034 & {$[\mathrm{~m}]$} \\
\hline$U_{\infty}$ & 14,1 & {$[\mathrm{~m} / \mathrm{s}]$} \\
\hline$\beta_{1}$ & 0,1 & {$[\mathrm{rad}]$} \\
\hline$\beta_{2}$ & 0,1 & {$[\mathrm{rad}]$} \\
\hline$m$ & 0,2 & {$[\mathrm{~kg}]$} \\
\hline$I_{1}$ & $6,31 \cdot 10^{-4}$ & {$\left[\mathrm{~kg} \mathrm{~m}^{2}\right]$} \\
\hline$I_{2}$ & $22,06 \cdot 10^{-4}$ & {$\left[\mathrm{~kg} \mathrm{~m}^{2}\right]$} \\
\hline$I_{3}$ & $26,32 \cdot 10^{-4}$ & {$\left[\mathrm{~kg} \mathrm{~m}^{2}\right]$} \\
\hline$k_{31}$ & 0 & {$[-]$} \\
\hline
\end{tabular}

\subsubsection{Parámetros generales del ejemplo numérico}

Salvo que se diga lo contrario, la orientación inicial del pararrotor será tal que los ejes móviles serán coincidentes con los inerciales. Esta orientación inicial se corresponde con una matriz de rotación (125) igual a la identidad. 


\subsubsection{Simulación numérica y modelo gráfico}

La simulación numérica de la dinámica del pararrotor se puede esquematizar a partir del siguiente ordenamiento, para cada paso de integración:

- cálculo de fuerzas y momentos generados por las palas,

- cómputo (integración numérica) de la dinámica del pararrotor y su orientación.

El modelo gráfico general de este proceso se muestra en la Figura 3.27.

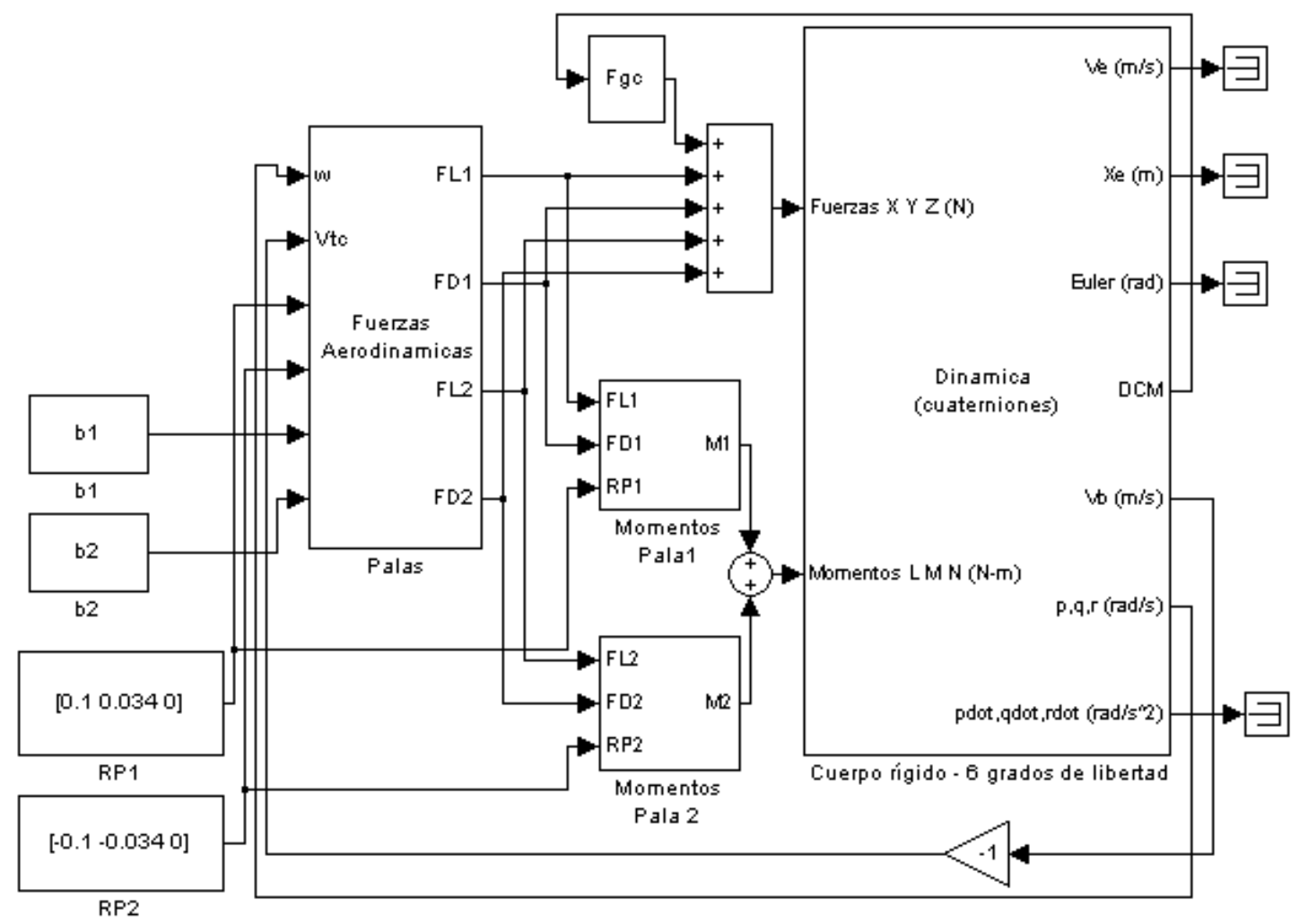

Figura 0.27 Esquema gráfico de cómputo del sistema para un pararrotor de 6 grados de libertad. 
La determinación de las fuerzas y momentos generados en las palas del pararrotor son la base de la simulación de su comportamiento dinámico. El proceso descripto es el utilizado para simular el comportamiento del pararrotor en sus distintas variantes de configuración, que se obtiene realizando la integración numérica de las ecuaciones diferenciales de su dinámica.

Se adopta para el cálculo numérico un paso de integración fijo, de 0,005 segundos, utilizando el método de Dormand \& Prince (1980).

Para la adopción del método de integración y tipo de paso se compararon los resultados después de 10 segundos de integración de un número de casos, con el resultado analítico que se muestra en la Tabla 3.6. En particular se consideraron métodos de la familia Runge-Kutta no adaptativos, vale decir de valor de paso de integración fijo. Dentro de la familia RungeKutta de métodos iterativos (J.C. Butcher, 2003) se consideró el método denominado como Dormand-Prince (de orden 5), el denominado específicamente como Runge-Kutta (de orden 4) y el Bogacki-Shampine (de orden 3).

Se adoptó una técnica de integración de paso fijo por simplicidad y velocidad, por cuanto no se le es requerido al algoritmo de cálculo el cómputo de la longitud de paso, generando como consecuencia márgenes mayores de error en la integración.

El tiempo de paso adoptado fue consecuencia del cálculo de valores de error para distintas variantes de paso de integración y método. Se justifica su elección a partir de comprobar que se garantizan márgenes de error menores a $10^{-4}$ al respecto de soluciones analíticas exactas. Se considera que la magnitud de estos márgenes es suficientemente pequeña como para impedir la aparición de soluciones de la dinámica del pararrotor asociadas al error, alejadas de los efectos propios de la mecánica del dispositivo. El estudio de la influencia de estos factores se muestra en el apartado que sigue.

\subsection{VALIDACIÓN}

Para la validación de la simulación de la dinámica del pararrotor se considerará el caso de un cuerpo rígido sólido con el movimiento libre con tensor de inercia cilíndrico (o cuerpo axilsimétrico) no sometido a momentos ni fuerzas exteriores, con condiciones iniciales de 
velocidad de translación nulas y determinada velocidad rotacional. Los resultados se comparan con la solución de problema clásico del movimiento sin momentos exteriores (Thomson (1986)).

Las condiciones iniciales del presente caso (que de ahora en adelante denominaremos Caso I) se muestran en la Tabla 3.4.

Tabla 0.4 Parámetros generales del ejemplo numérico.

\begin{tabular}{|c|cc|}
\hline \multicolumn{3}{|c|}{ Parámetros generales y condiciones iniciales } \\
\hline$I_{1}$ & $6,31 \cdot 10^{-4}$ & $\mathrm{~kg} \mathrm{~m}^{2}$ \\
\hline$I_{2}$ & $6,31 \cdot 10^{-4}$ & $\mathrm{~kg} \mathrm{~m}^{2}$ \\
\hline$I_{3}$ & $22,25 \cdot 10^{-4}$ & $\mathrm{~kg} \mathrm{~m}^{2}$ \\
\hline$\omega_{\text {inicial } 1}$ & 0 & $1 / \mathrm{s}$ \\
\hline$\omega_{\text {inicial } 2}$ & 1 & $1 / \mathrm{s}$ \\
\hline$\omega_{\text {inicial } 3}$ & 5 & $1 / \mathrm{s}$ \\
\hline
\end{tabular}

Inicialmente, en $t=0$, el momento cinético se encuentra dado por:

$\mathbf{h}_{0}=I_{2} \omega_{\text {inicial } 2} \mathbf{e}_{2}+I_{3} \omega_{\text {inicial } 3} \mathbf{e}_{3}$.

Como el momento cinético se mantiene constante en el sistema en ausencia de momentos exteriores actuantes, si $\mathbf{h}_{0} \equiv$ eje $\mathbf{Z}$ el ángulo $\theta$ se mantiene constante en el tiempo. Por otro lado se tiene que:

$\tan \theta_{\text {inicial }}=\frac{I_{2} \omega_{\text {inicial 2 }}}{I_{3} \omega_{\text {inicial 3 }}}$, 
A continuación se resume brevemente la solución del Caso I.

Las ecuaciones de Euler son:

$0=\frac{\mathrm{d} \omega_{1}}{\mathrm{~d} t}-\left(\frac{I_{2}-I_{3}}{I_{1}}\right) \omega_{3} \omega_{2}$

$0=\frac{\mathrm{d} \omega_{2}}{\mathrm{~d} t}+\left(\frac{I_{1}-I_{3}}{I_{2}}\right) \omega_{3} \omega_{1}$

$0=\frac{\mathrm{d} \omega_{3}}{\mathrm{~d} t}$

Esto equivale a decir que la rotación sobre el eje 3, $\omega_{3}$ permanecerá constante. Por conveniencia se denomina:

$\lambda=\frac{I_{3}-I_{2}}{I_{2}} \omega_{3}$,

que en este caso es $\lambda=12,631 \mathrm{rad} / \mathrm{s}$.

Definimos a $\omega_{12}$ en la siguiente expresión:

$\omega_{12}=\sqrt{\omega_{1}^{2}+\omega_{2}^{2}}$

Como el momento cinético se mantiene constante en virtud de que no existen momentos actuando sobre el cuerpo, y como $\omega_{3}$ permanece constante, entonces, combinando (149) y (150) se obtiene:

$\omega_{12}=\sqrt{\omega_{1}^{2}+\omega_{2}^{2}}=$ constante $=\omega_{\text {inicial } 2}$.

Si tomamos la primera y la segunda ecuación de la dinámica rotacional (149) y (150), tenemos: 
$0=\frac{\mathrm{d} \omega_{1}}{\mathrm{~d} t}+\lambda \omega_{2}$

$0=\frac{\mathrm{d} \omega_{2}}{\mathrm{~d} t}-\lambda \omega_{1}$,

pues $I_{1}=I_{2}$.

Puede comprobarse que la solución analítica de este sistema es:

$\omega_{1}(t)=-\omega_{\text {inicial } 2} \sin (\lambda t)$,

$\omega_{2}(t)=\omega_{\text {inicial } 2} \cos (\lambda t)$. 


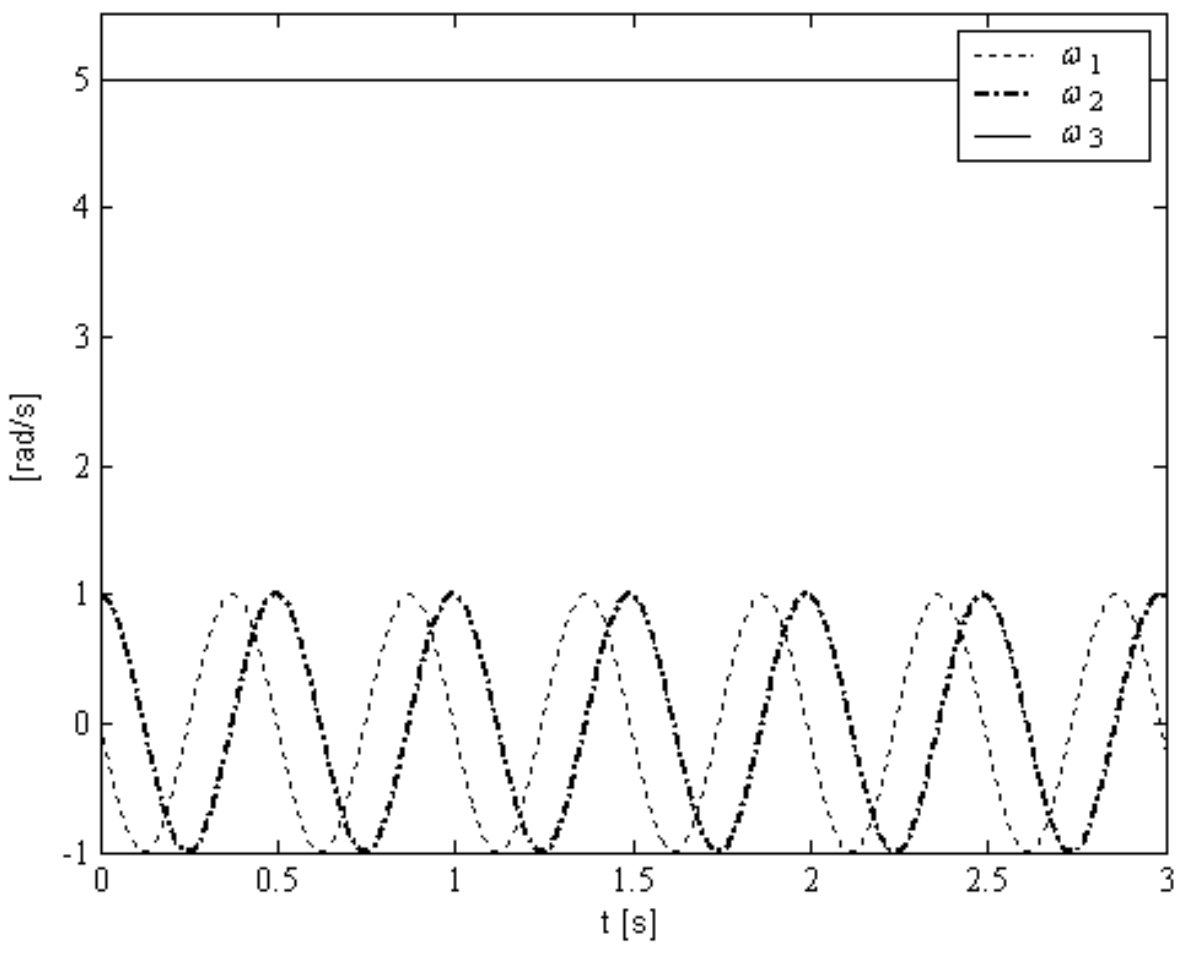

Figura 0.28 Variación con el tiempo, $t$, de las velocidades angulares $\omega_{1}, \omega_{2}$ y $\omega_{3}$, calculadas numéricamente en el caso de la dinámica de un cuerpo rígido sin fuerzas ni momentos exteriores.

Si se considera la orientación de la terna móvil al respecto de la terna inercial para el presente caso, en función de los ángulos de Euler $\psi, \theta$ y $\varphi$ (con orden de rotación 313 ), es posible establecer el movimiento del pararrotor al respecto de la terna inercial.

Las ecuaciones que vinculan los ángulos de Euler con las velocidades angulares en ejes de la terna móvil pueden expresarse de la siguiente manera:

$$
\begin{aligned}
& \omega_{1}=\frac{\mathrm{d} \psi}{\mathrm{d} t} \sin \theta \sin \varphi+\frac{\mathrm{d} \theta}{\mathrm{d} t} \cos \varphi, \\
& \omega_{2}=\frac{\mathrm{d} \psi}{\mathrm{d} t} \sin \theta \cos \varphi-\frac{\mathrm{d} \theta}{\mathrm{d} t} \sin \varphi,
\end{aligned}
$$


$\omega_{3}=\frac{\mathrm{d} \varphi}{\mathrm{d} t}+\frac{\mathrm{d} \psi}{\mathrm{d} t} \cos \theta$

Como el momento cinético se encuentra fijo en el espacio, y $\theta$ permanece constante $(\mathrm{d} \theta / \mathrm{d} t$ =0) manteniendo la orientación inicial, las ecuaciones (158), (159) y (160) resultan:

$\omega_{1}=\frac{\mathrm{d} \psi}{\mathrm{d} t} \sin \theta \sin \varphi$

$\omega_{2}=\frac{\mathrm{d} \psi}{\mathrm{d} t} \sin \theta \cos \varphi$

$\omega_{3}=\frac{\mathrm{d} \varphi}{\mathrm{d} t}+\frac{\mathrm{d} \psi}{\mathrm{d} t} \cos \theta$

Diferenciando las ecuaciones (161), (162) y (163) y reemplazando en (149), siguiendo el caso propuesto también por Thompson (1986) se tiene que:

$\frac{\mathrm{d} \psi}{\mathrm{d} t}=\frac{I_{3}}{I_{1}-I_{3}} \frac{1}{\cos \theta} \frac{\mathrm{d} \varphi}{\mathrm{d} t}$

Finalmente se obtiene que:

$$
\begin{aligned}
& \frac{\mathrm{d} \varphi}{\mathrm{d} t}=\omega_{3} \frac{I_{1}-I_{3}}{I_{1}}, \\
& \frac{\mathrm{d} \psi}{\mathrm{d} t}=\frac{\omega_{3}}{\cos \theta} \frac{I_{3}}{I_{1}} .
\end{aligned}
$$

Como $\omega_{3}$ es constante se tiene que $\psi$, y $\varphi$ son funciones lineales del tiempo. Para el caso numérico presente, donde $\psi_{\text {inicial }}=\varphi_{\text {inicial }}=0$, los ángulos de Euler resultan:

$$
\begin{aligned}
& \theta(t)=\theta_{\text {inicial }}=3,25^{\circ}=0,057, \\
& \varphi(t)=-12,631 t
\end{aligned}
$$


$\psi(t)=17,659 t$

Con el fin de realizar una comparación de la solución numérica con la solución analítica se resuelven las ecuaciones de la dinámica por medio de la integración numérica mediante el método Dormand \& Prince (1980), con paso de integración fijo de 0,005 segundos.

La solución numérica, que se muestra en la Figura 3.28, corresponde a lo esperado según su solución analítica. Haciendo una comparación de valores numéricos (ver Tabla 3.5) se aprecia que la solución numérica coincide prácticamente con la analítica. Se ha comparado el resultado numérico (velocidades angulares que se denominan $\omega_{i N}$ ) y el resultado analítico (velocidades angulares que se denominan $\omega_{i T}$ ) para valores de tiempo de integración (variable t) monótonamente crecientes. Con este objetivo se define el parámetro $E_{N}$, como medida del error relativo, dado por:

$$
E_{N}=\sqrt{\left(\omega_{1 T}-\omega_{1 N}\right)^{2}+\left(\omega_{2 T}-\omega_{2 N}\right)^{2}+\left(\omega_{3 T}-\omega_{3 N}\right)^{2}} .
$$

Tabla 0.5 Comparativa entre resultados numéricos, $\omega_{\mathrm{iN}}, \mathrm{y}$ analíticos, $\omega_{\mathrm{i} T},\left(\omega_{3 T}=5,0000\right)$.

\begin{tabular}{|c|c|c|c|c|c|c|c|}
\hline Variable & \multicolumn{4}{|c|}{ Resultado numérico } & \multicolumn{2}{c|}{$\begin{array}{c}\text { Resultado } \\
\text { analítico }\end{array}$} & Error \\
\hline $\begin{array}{c}t \\
{[\mathrm{~s}]}\end{array}$ & $\begin{array}{c}\omega_{1 N} \\
{[\mathrm{rad} / \mathrm{s}]}\end{array}$ & $\begin{array}{c}\omega_{2 N} \\
{[\mathrm{rad} / \mathrm{s}]}\end{array}$ & $\begin{array}{c}\omega_{3} \\
{[\mathrm{rad} / \mathrm{s}]}\end{array}$ & $\begin{array}{c}\left(\omega_{12 \text { inicial }}-\omega_{12}\right) \\
{\left[\mathrm{rad} / \mathrm{s} \cdot 10^{-5}\right]}\end{array}$ & $\begin{array}{c}\omega_{1 T} \\
{[\mathrm{rad} / \mathrm{s}]}\end{array}$ & $\begin{array}{c}\omega_{2 T} \\
{[\mathrm{rad} / \mathrm{s}]}\end{array}$ & $\begin{array}{c}E_{N} \\
{\left[\mathrm{rad} / \mathrm{s} \cdot 10^{-5}\right]}\end{array}$ \\
\hline 0,02 & 0,9683 & 0,2499 & 5,0000 & $-2,7$ & 0,9683 & 0,2499 & 5,3 \\
\hline 0,04 & 0,8751 & 0,4840 & 5,0000 & $-2,8$ & 0,8751 & 0,4840 & 3,8 \\
\hline 0,06 & 0,7263 & 0,6874 & 5,0000 & $-1,5$ & 0,7263 & 0,6874 & 4,7 \\
\hline 0,08 & 0,5315 & 0,8471 & 5,0000 & $-3,5$ & 0,5315 & 0,8471 & 3,7 \\
\hline 0,10 & 0,3029 & 0,9530 & 5,0000 & 2,1 & 0,3029 & 0,9530 & 2,9 \\
\hline 1,00 & 0,9979 & 0,0643 & 5,0000 & 3,1 & 0,9979 & 0,0643 & 4,1 \\
\hline 2,00 & 0,9917 & 0,1284 & 5,0000 & 2,2 & 0,9917 & 0,1284 & 2,4 \\
\hline
\end{tabular}




\begin{tabular}{|c|c|c|c|c|c|c|c|}
\hline Variable & \multicolumn{4}{|c|}{ Resultado numérico } & \multicolumn{2}{c|}{$\begin{array}{c}\text { Resultado } \\
\text { analítico }\end{array}$} & Error \\
\hline $\begin{array}{c}t \\
{[\mathrm{~s}]}\end{array}$ & $\begin{array}{c}\omega_{1 N} \\
{[\mathrm{rad} / \mathrm{s}]}\end{array}$ & $\begin{array}{c}\omega_{2 N} \\
{[\mathrm{rad} / \mathrm{s}]}\end{array}$ & $\begin{array}{c}\omega_{3} \\
{[\mathrm{rad} / \mathrm{s}]}\end{array}$ & $\begin{array}{c}\left(\omega_{12 \text { inicial }}-\omega_{12}\right) \\
{\left[\mathrm{rad} / \mathrm{s} \cdot 10^{-5}\right]}\end{array}$ & $\begin{array}{c}\omega_{1 T} \\
{[\mathrm{rad} / \mathrm{s}]}\end{array}$ & $\begin{array}{c}\omega_{2 T} \\
{[\mathrm{rad} / \mathrm{s}]}\end{array}$ & $\begin{array}{c}E_{N} \\
{\left[\mathrm{rad} / \mathrm{s} \cdot 10^{-5}\right]}\end{array}$ \\
\hline 3 & 0,9814 & 0,1919 & 5,0000 & 1,4 & 0,9814 & 0,1919 & 2,6 \\
\hline 5 & 0,9486 & 0,3163 & 5,0000 & 5,6 & 0,9486 & 0,3163 & 6,2 \\
\hline 10 & 0,7999 & 0,6002 & 5,0000 & $-4,0$ & 0,7999 & 0,6002 & 4,5 \\
\hline 60 & $-0,7512$ & $-0,6600$ & 5,0000 & 4,9 & $-0,7512$ & $-0,6600$ & 4,9 \\
\hline
\end{tabular}

Se puede observar que el error se mantiene menor a 0,0001, independientemente del transcurso del tiempo de integración.

El resultado de la simulación numérica arroja los siguientes valores constantes que se registran en el transcurso del tiempo de simulación:

$\theta_{\text {medio }}=0,0567$,

$\frac{\mathrm{d} \varphi}{\mathrm{d} t}=-12,6307$,

$\frac{\mathrm{d} \psi}{\mathrm{d} t}=17,6591$

Estos valores son coincidentes con los determinados a partir de la solución analítica.

La Figura 3.29 muestra el resultado de la simulación numérica del presente caso para 1 segundo de tiempo de integración, en términos de los ángulos $\psi, \theta$, y $\varphi$. 


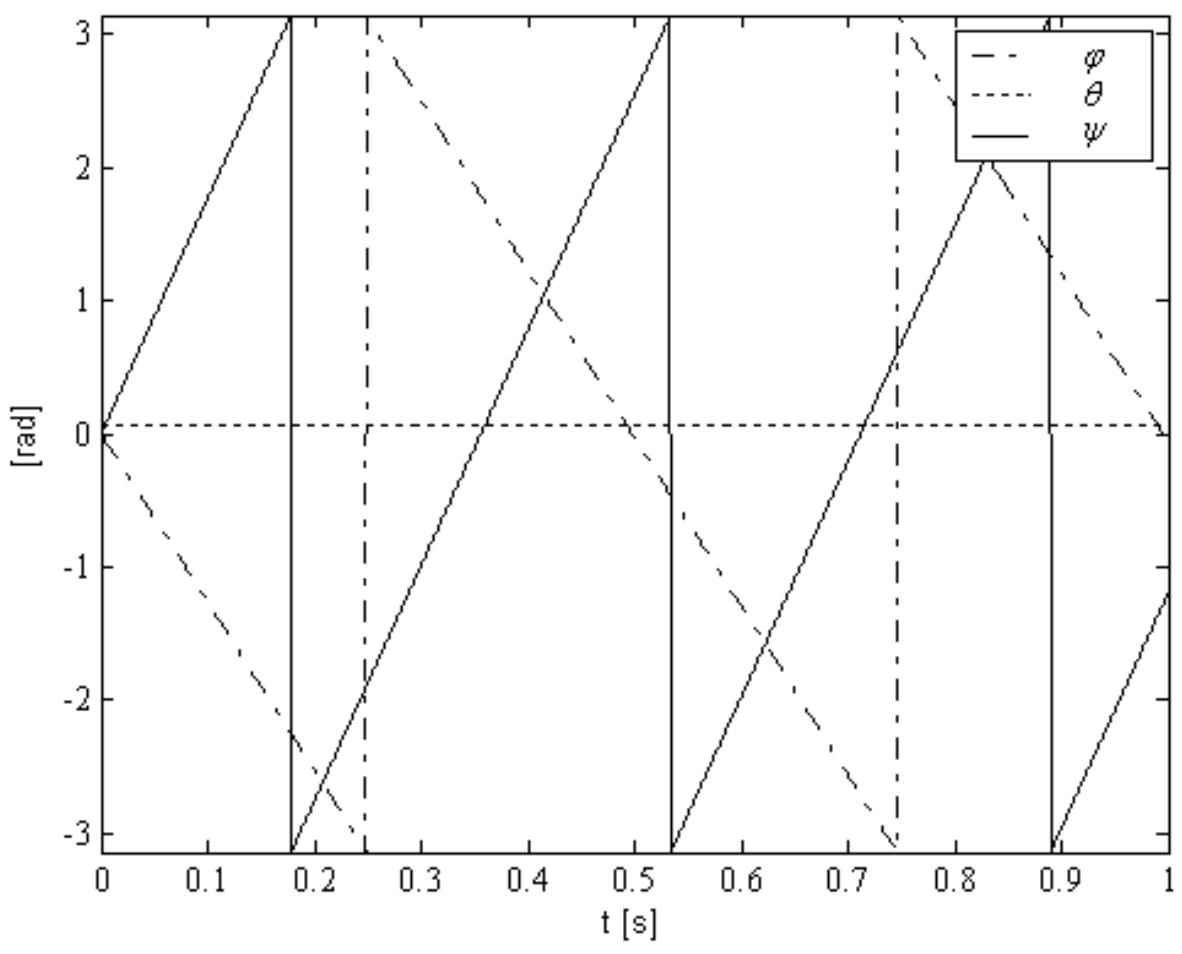

Figura 0.29 Variación con el tiempo, $t$, de los ángulos de Euler $\varphi, \theta, \psi$, resultado de la simulación numérica para el caso de validación, para un dominio entre $-\pi \mathrm{y} \pi$.

Como se mencionó anteriormente, sobre la base de la comparación de los resultados de métodos de paso de integración fija de distinto orden se adoptó el denominado como Dormand-Prince (de orden 5).

El orden del método de integración da cuenta de la cantidad de coeficientes que es necesario determinar para el cómputo numérico de los pasos temporales de una determinada función diferencial ordinaria, significando que el error por paso es del orden de la quinta potencia del ancho de paso.

En cuanto a la influencia del método de integración, en la Tabla 3.6 se muestran los resultados obtenidos. 
Tabla 0.6 Comparativa entre distintos métodos de integración numérica y paso de integración.

\begin{tabular}{|c|c|c|c|c|}
\hline \multirow{2}{*}{ Método } & $\begin{array}{c}\text { Paso } \\
{[\mathbf{s}]}\end{array}$ & $\begin{array}{c}\boldsymbol{\omega}_{\mathbf{1}} \\
{[\mathbf{r a d} / \mathbf{s}]}\end{array}$ & $\begin{array}{c}\boldsymbol{\omega}_{2} \\
{[\mathbf{r a d} / \mathbf{s}]}\end{array}$ & $\begin{array}{c}\boldsymbol{E}_{\boldsymbol{N}} \\
{[\mathbf{r a d} / \mathbf{s}]}\end{array}$ \\
\hline \multirow{3}{*}{ Dormand Prince } & 0,005 & 0,7999 & 0,6002 & 0,00004002 \\
\cline { 2 - 5 } & 0,050 & 0,7962 & 0,6017 & 0,00201336 \\
\cline { 2 - 5 } & 0,100 & 0,7396 & 0,7560 & 0,05761248 \\
\hline \multirow{3}{*}{ Runge Kutta } & 0,005 & 0,7999 & 0,6002 & 0,00004002 \\
\cline { 2 - 5 } & 0,050 & 0,8073 & 0,4405 & 0,08034053 \\
\cline { 2 - 5 } & 0,100 & 0,0765 & $-0,0632$ & 0,90073465 \\
\hline \multirow{3}{*}{ Bogacki Shampine } & 0,005 & 0,7998 & 0,5995 & 0,00045996 \\
\cline { 2 - 5 } & 0,050 & 0,8847 & 0,3020 & 0,06517483 \\
\cline { 2 - 5 } & 0,100 & $-0,0046$ & $-0,0026$ & 0,99464384 \\
\hline
\end{tabular}

Se puede observar que, si se establece como límite de error un valor de $10^{-4}$, el paso de integración deberá ser menor a 0,005 segundos. Adicionalmente se puede observar en la Figura 3.30 el importante crecimiento del error con el aumento del paso de integración, y por tanto la dependencia de la calidad de los resultados del método con el valor de paso de integración. Todos los métodos adoptados satisfacen el requerimiento impuesto. No obstante se implementó el método de Dormand Prince (con paso fijo de 0,0001 segundos), por tratarse del método de mayor orden de los tres ensayados cuyo coste computacional relativo no representa un limitante para su utilización para la potencia computacional disponible al momento. 


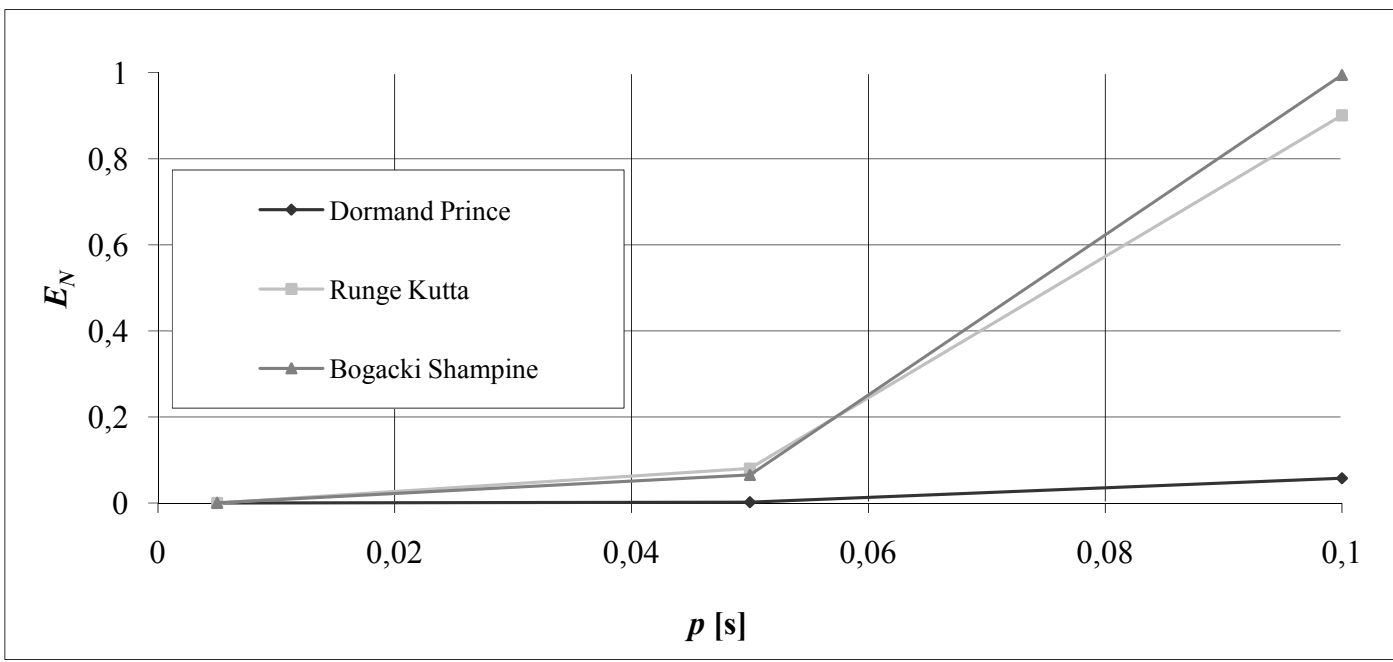

Figura 0.30 Variación con el paso de integración del error, $E_{N}$, cometido en el cálculo al respecto del resultado analítico, considerando las velocidades angulares calculadas numéricamente del caso de la dinámica de un cuerpo rígido sin momentos exteriores, para distintos valores de paso de integración, $p$.

\subsection{ANÁLISIS DE CASOS}

En el presente apartado se considerarán los 4 casos propuestos en el Capítulo 2, para comprobar en términos numéricos, sobre una configuración física concreta y posible, las conclusiones a las que se arribaron. Estos casos se presentan en la Tabla 3.7.

Se considerarán condiciones de vuelo cercanas al equilibrio del sistema. La determinación analítica de la relación entre rotación del pararrotor sobre el eje 3 y la velocidad de caída fue realizada a partir de las siguientes expresiones, sobre la base de las hipótesis adoptadas en el desarrollo del modelo analítico y una configuración de vuelo simétrica, con el eje 3 alineado con el eje $\mathbf{Z}$ :

$C_{L \alpha} \Theta\left(\Theta-\beta_{o}\right)=C_{D}$ 
$2 C_{L a}\left(\Theta-\beta_{o}\right)=\frac{m g}{\frac{1}{2} \rho S r_{11}{ }^{2} \omega_{3}{ }^{2}}$

El análisis de la estabilidad dinámica de los casos se realiza sobre la base del análisis de la evolución del ángulo de nutación en el tiempo, puesto que este ángulo caracteriza las soluciones de equilibrio del sistema en términos de la orientación relativa de la terna asociada al pararrotor con la terna inercial. Se consideran como soluciones estables a todas aquellas soluciones que se encuentren en un entorno acotado de un punto de equilibrio, y además, sean funcionalmente aceptables para el objeto práctico del dispositivo.

Los casos que se estudiarán se resumen en la Tabla 3.7.

Tabla 0.7 Parámetros generales del ejemplo numérico

\begin{tabular}{|c|c|c|c|}
\hline Caso & $\begin{array}{c}\text { Relaciones de los } \\
\text { momentos de } \\
\text { inercia }\end{array}$ & $\begin{array}{c}\text { Tabla de } \\
\text { datos }\end{array}$ & $\begin{array}{l}\text { Figura con } \\
\text { resultados }\end{array}$ \\
\hline A & $I_{3}>I_{2}, I_{1}$ & $\begin{array}{c}\text { Tabla } 3.8 \\
\text { a } \\
\text { Tabla } 3.25\end{array}$ & $\begin{array}{c}\text { Figura } 3.31 \\
\quad \mathrm{a} \\
\text { Figura } 3.58\end{array}$ \\
\hline B & $I_{2}>I_{3}>I_{1}$ & $\begin{array}{c}\text { Tabla } 3.26 \\
\text { a } \\
\text { Tabla } 3.28 \\
\end{array}$ & Figura 3.59 \\
\hline $\mathrm{C}$ & $I_{1}>I_{3}>I_{2}$ & $\begin{array}{c}\text { Tabla } 3.29 \\
\text { a } \\
\text { Tabla } 3.30 \\
\end{array}$ & Figura 3.60 \\
\hline $\mathrm{D}$ & $I_{1}, I_{2}>I_{3}$ & $\begin{array}{c}\text { Tabla } 3.31 \\
\text { a } \\
\text { Tabla } 3.35\end{array}$ & Figura 3.61 \\
\hline
\end{tabular}

Sobre cada uno de los casos descritos se estudiará el efecto de la variación del parámetro $k_{31}$, haciendo que éste tome distintos valores sobre la base de distintas configuraciones del dispositivo.

Estos ejemplos numéricos se realizan sobre la base de una serie de parámetros que establecen una configuración de base, que se presentan en la Tabla 3.8. Se han seleccionado estos 
parámetros de manera que resulten representativos y verosímiles en términos tecnológicos. No obstante es posible encontrar variantes del caso base con valores de $k_{31}$ tecnológicamente en principio inviables con el fin de evidenciar la evolución del comportamiento dinámico del pararrotor con $k_{31}$.

Tabla 0.8 Configuración básica del ejemplo numérico.

\begin{tabular}{|c|cc|}
\hline \multicolumn{3}{|c|}{ Parámetros generales } \\
\hline$C_{L \alpha}$ & 1,35 & $1 / \mathrm{rad}$ \\
\hline$C_{D}$ & 0,15 & - \\
\hline$\rho$ & 1,21 & $\mathrm{~kg} / \mathrm{m}^{3}$ \\
\hline$S$ & 0,0254 & $\mathrm{~m}^{2}$ \\
\hline$r_{11}, r_{21}$ & 0,037 & $\mathrm{~m}$ \\
\hline$r_{12}, r_{22}$ & 0,016 & $\mathrm{~m}$ \\
\hline$\beta_{1}, \beta_{2}$ & 0,07 & $\mathrm{rad}$ \\
\hline
\end{tabular}

Se presentan distintas variantes de cada caso considerando los parámetros $N_{e}$, $k_{e}$, con el objeto de visualizar la estabilidad del sistema asociada al valor que toman estos parámetros.

Las condiciones iniciales se presentan en la Tabla 3.9 para los ejemplos numéricos considerando la configuración básica definida por los valores de los parámetros recogidos en la Tabla 3.8.

Tabla 0.9 Condiciones iniciales de los ejemplos numéricos.

\begin{tabular}{|c|c|}
\hline \multicolumn{2}{|c|}{ Condiciones iniciales } \\
\hline$\omega_{1}$ & $0 \mathrm{rad} / \mathrm{s}$ \\
\hline
\end{tabular}




\begin{tabular}{|c|c|}
\hline$\omega_{2}$ & $0 \mathrm{rad} / \mathrm{s}$ \\
\hline$\omega_{3}$ & $\omega_{\text {eq } 3}$ \\
\hline
\end{tabular}

La duración del cálculo de los diferentes sistemas se fijará en un tiempo tal que se verifique que se ha alcanzado una solución de equilibrio del sistema. Dicha evolución permitirá describir y comparar la estabilidad del cada situación considerada.

\subsubsection{Caso A - Eje principal mayor próximo al eje de giro}

El presente caso se basa en el cumplimiento de la condición que $I_{3}>I_{2}, I_{1}$.

Para el cumplimiento de la condición se adoptan los momentos de inercia que se presentan en la Tabla 3.10 como caso base.

Tabla 0.10 Momentos de inercia para $I_{3}>I_{2}, I_{1}$.

\begin{tabular}{|c|c|}
\hline \multicolumn{2}{|c|}{ Momentos de inercia } \\
\hline$I_{1}$ & $5,4 \cdot 10^{-6} \mathrm{~kg} \mathrm{~m}^{2}$ \\
\hline$I_{2}$ & $21,2 \cdot 10^{-6} \mathrm{~kg} \mathrm{~m}^{2}$ \\
\hline$I_{3}$ & $25,9 \cdot 10^{-6} \mathrm{~kg} \mathrm{~m}^{2}$ \\
\hline
\end{tabular}

Para los momentos de inercia presentados se simuló numéricamente el comportamiento del pararrotor con el fin de hallar la solución de equilibrio para el caso en que los ángulos de paso de las palas sean idénticos $\left(\beta_{1}=\beta_{2}\right)$ y para el caso de distintos ángulos de paso de palas $\left(\beta_{1} \neq\right.$ $\beta_{2}$ ) para distintas relaciones del parámetro $k_{31}$. En estas simulaciones el ángulo inicial de nutación fue nulo, vale decir de la posición de alineación del eje 3 del pararrotor con el eje vertical inercial. Los resultados se presentan en las Tablas 3.11 a 3.18. Las condiciones finales fueron aquellas asociadas a la solución de equilibrio. 
Los valores obtenidos del modelo analítico corresponden al cómputo de las ecuaciones del modelo linealizado donde son considerados sólo los términos con órdenes de magnitud mayores, mientras que aquellos asociados a la simulación numérica supone el cómputo de las ecuaciones completas del desarrollo analítico. Las diferencias entre los resultados que se presentan obviamente responden al cómputo de los órdenes de menor magnitud que diferencian a estos dos modelos mencionados.

La Tabla 3.11 muestra que para $k_{31}=0$, la solución de equilibrio estable única es aquella donde el ángulo de nutación de equilibrio es 0 o muy cercano a 0 radianes; y las velocidades angulares $\omega_{e q 1}, \omega_{e q 2}$, son mucho menores a la unidad. Se puede ver que el modelo analítico y la simulación numérica son congruentes en la estimación de las variables asociadas a esta solución de equilibrio. Por otro lado también se puede observar de la Figura 3.31 que en la medida que aumenta la diferencia entre $\beta_{1}$ y $\beta_{2}$, aumenta el error entre la estimación del modelo analítico y los valores obtenidos de la simulación.

Tabla 0.11 Soluciones de equilibrio para distintas relaciones de paso de palas con $k_{31}=0$.

\begin{tabular}{|c|c|c|c|c|c|}
\hline Casos & $\begin{array}{c}\omega_{e q 1} \\
{[\mathrm{rad} / \mathrm{s}]}\end{array}$ & $\begin{array}{c}\omega_{e q 2} \\
{[\mathrm{rad} / \mathrm{s}]}\end{array}$ & $\begin{array}{c}\omega_{e q 3} \\
{[\mathrm{rad} / \mathrm{s}]}\end{array}$ & $\begin{array}{c}U \\
{[\mathbf{m} / \mathbf{s}]}\end{array}$ & $\begin{array}{c}\theta_{e} \\
{[\mathbf{r a d}]}\end{array}$ \\
\hline \multirow{2}{*}{$\begin{array}{l}\beta_{1}=0,07 \\
\beta_{2}=0,07\end{array}$} & $0,0000(1)$ & $0,0000(1)$ & $292,8(3)$ & 4,57 (3) & $0,000(1)$ \\
\hline & $0,0000(2)$ & $0,0000(2)$ & $292,8(2)$ & 4,57 (2) & $0,000(2)$ \\
\hline \multirow{2}{*}{$\begin{array}{l}\beta_{1}=0,07 \\
\beta_{2}=0,09\end{array}$} & $0,0303(1)$ & 0,0581 (1) & $294,0(3)$ & $4,66(3)$ & $2,229 \cdot 10^{-4}(1)$ \\
\hline & $0,0327(2)$ & $0,0618(2)$ & $294,0(2)$ & $4,66(2)$ & $2,377 \cdot 10^{-4}(2)$ \\
\hline \multirow{2}{*}{$\begin{array}{l}\beta_{1}=0,07 \\
\beta_{2}=0,14\end{array}$} & $0,1072(1)$ & $0,2056(1)$ & 297,2 (3) & $4,88(3)$ & $7,802 \cdot 10^{-4}(1)$ \\
\hline & 0,1165 (2) & $0,2198(2)$ & 297,2 (2) & $4,88(2)$ & $8,370 \cdot 10^{-4}(2)$ \\
\hline \multirow{2}{*}{$\begin{array}{l}\beta_{1}=0,07 \\
\beta_{2}=0,19\end{array}$} & $0,1856(1)$ & $0,3561(1)$ & $300,2(3)$ & $5,11(3)$ & $1,338 \cdot 10^{-3}(1)$ \\
\hline & $0,2030(2)$ & $0,3830(2)$ & 300,2 (2) & $5,11(2)$ & $1,444 \cdot 10^{-3}(2)$ \\
\hline $\begin{array}{l}\beta_{1}=0,07 \\
\beta_{2}=0,21\end{array}$ & $0,2174(1)$ & $0,4170(1)$ & $301,4(3)$ & $5,21(3)$ & $1,560 \cdot 10^{-3}(1)$ \\
\hline
\end{tabular}




\begin{tabular}{|l|c|c|c|c|c|}
\hline & $0,2384(2)$ & $0,4497(2)$ & $301,4(2)$ & $5,21(2)$ & $1,689 \cdot 10^{-3}(2)$ \\
\hline $\begin{array}{l}\beta_{1}=0,07 \\
\beta_{2}=0,25\end{array}$ & $0,2816(1)$ & $0,5403(1)$ & $303,7(3)$ & $5,41(3)$ & $2,006 \cdot 10^{-3}(1)$ \\
\hline
\end{tabular}

(1) Valores obtenidos del modelo analítico, (2) valores obtenidos de la simulación numérica y (3) valores adoptados como dato de entrada.

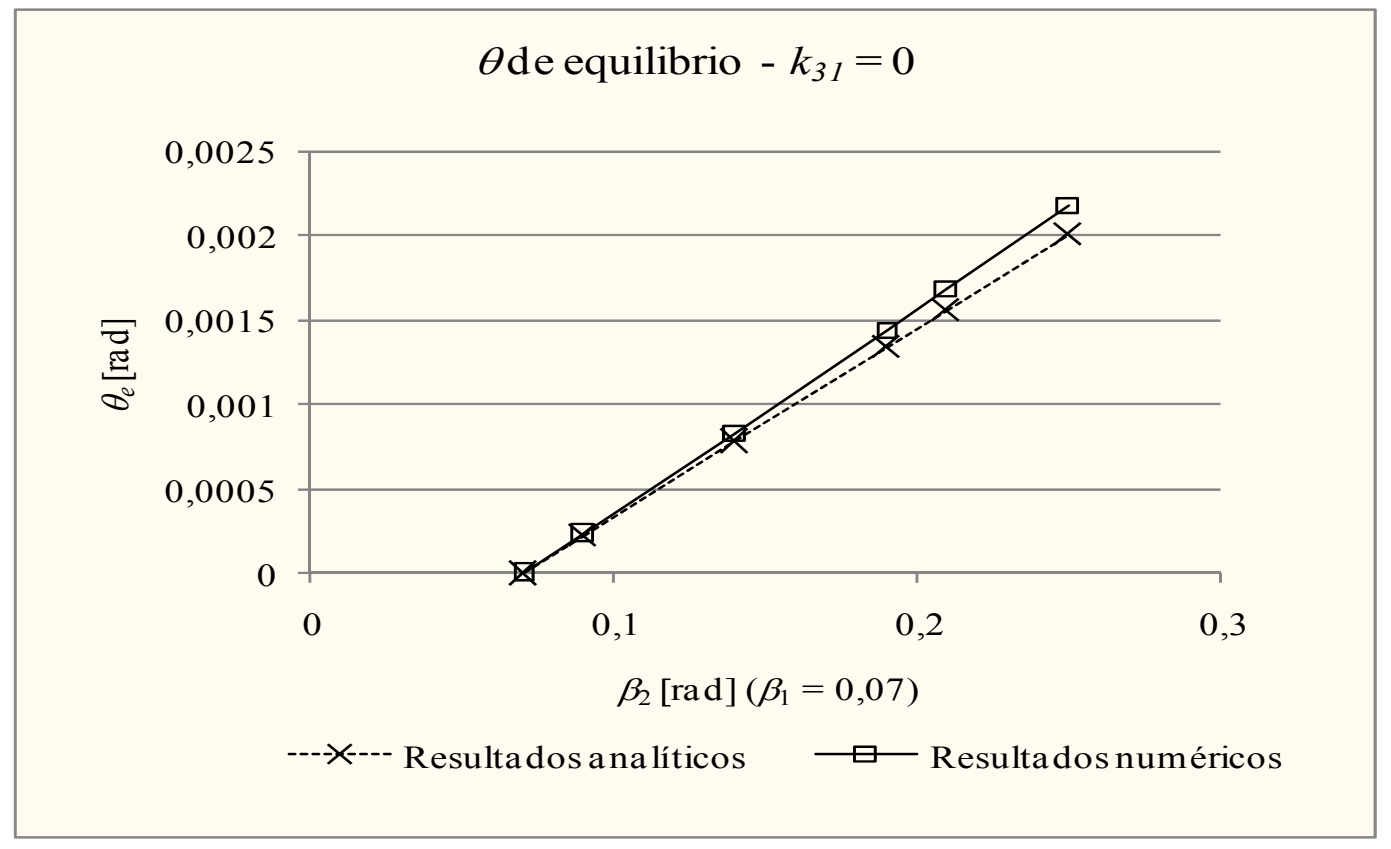

Figura 0.31 Variación del ángulo de nutación de equilibrio, $\theta_{e}$, en función de $\beta_{2}$ (con $\beta_{1}=$ 0,07 y $\left.k_{31}=0\right)$, para el modelo analítico y para la simulación numérica.

La Tabla 3.12 muestra que para $k_{31}=0,1$, al igual que el caso donde $k_{31}=0$, la solución de equilibrio estable que se presenta es única, donde el ángulo de nutación de equilibrio es 0 o 
muy cercano a 0 radianes; y las velocidades angulares $\omega_{e q 1}, \omega_{e q 2}$, son mucho menores a la unidad. Se puede ver que el modelo analítico y la simulación numérica son congruentes en la estimación de las variables asociadas a esta solución de equilibrio. Por otro lado también se puede observar de la Figura 3.32 que en la medida que aumenta la diferencia entre $\beta_{1}$ y $\beta_{2}$, aumenta el error entre la estimación del modelo analítico y los valores obtenidos de la simulación. También se puede observar que la estimación del modelo analítico es siempre menor a lo arrojado por la simulación numérica en términos del ángulo de nutación y que el valor de dicho ángulo es cercano a 0 .

Tabla 0.12 Soluciones de equilibrio para distintas relaciones de paso de palas con $k_{31}=0,1$.

\begin{tabular}{|c|c|c|c|c|c|}
\hline Casos & $\begin{array}{c}\omega_{e q 1} \\
{[\mathrm{rad} / \mathrm{s}]}\end{array}$ & $\begin{array}{c}\omega_{e q 2} \\
{[\mathrm{rad} / \mathrm{s}]}\end{array}$ & $\begin{array}{c}\omega_{e q 3} \\
{[\mathrm{rad} / \mathrm{s}]}\end{array}$ & $\begin{array}{c}U \\
{[\mathrm{~m} / \mathrm{s}]}\end{array}$ & $\begin{array}{c}\theta_{e} \\
{[\mathbf{r a d}]}\end{array}$ \\
\hline \multirow{2}{*}{$\begin{array}{l}\beta_{1}=0,07 \\
\beta_{2}=0,07\end{array}$} & $0,0000(1)$ & $0,0000(1)$ & $292,8(3)$ & $4,57(3)$ & $0,000(1)$ \\
\hline & $0,0000(2)$ & $0,0000(2)$ & $292,8(2)$ & $4,57(2)$ & $0,000(2)$ \\
\hline \multirow{2}{*}{$\begin{array}{l}\beta_{1}=0,07 \\
\beta_{2}=0,09\end{array}$} & $0,0302(1)$ & $0,0520(1)$ & $294,0(3)$ & $4,66(3)$ & $2,046 \cdot 10^{-4}(1)$ \\
\hline & 0,0327 (2) & $0,0554(2)$ & $294,0(2)$ & $4,66(2)$ & $2,188 \cdot 10^{-4}(2)$ \\
\hline \multirow{2}{*}{$\begin{array}{l}\beta_{1}=0,07 \\
\beta_{2}=0,14\end{array}$} & 0,1069 (1) & $0,1832(1)$ & 297,2 (3) & $4,88(3)$ & $7,137 \cdot 10^{-4}(1)$ \\
\hline & $0,1162(2)$ & $0,1964(2)$ & 297,2 (2) & $4,88(2)$ & $7,679 \cdot 10^{-4}(2)$ \\
\hline \multirow{2}{*}{$\begin{array}{l}\beta_{1}=0,07 \\
\beta_{2}=0,19\end{array}$} & 0,1851 (1) & $0,3159(1)$ & $300,2(3)$ & $5,11(3)$ & $1,220 \cdot 10^{-3}(1)$ \\
\hline & $0,2025(2)$ & $0,3407(2)$ & $300,2(2)$ & $5,11(2)$ & $1,321 \cdot 10^{-3}(2)$ \\
\hline \multirow{2}{*}{$\begin{array}{l}\beta_{1}=0,07 \\
\beta_{2}=0,21\end{array}$} & $0,2168(1)$ & $0,3693(1)$ & $301,4(3)$ & $5,21(3)$ & $1,421 \cdot 10^{-3}(1)$ \\
\hline & $0,2378(2)$ & $0,3995(2)$ & $301,4(2)$ & $5,21(2)$ & $1,543 \cdot 10^{-3}(2)$ \\
\hline \multirow{2}{*}{$\begin{array}{l}\beta_{1}=0,07 \\
\beta_{2}=0,25\end{array}$} & 0,2809 (1) & 0,4767 (1) & 303,7 (3) & $5,41(3)$ & $1,822 \cdot 10^{-3}(1)$ \\
\hline & $0,3098(2)$ & $0,5185(2)$ & 303,7 (2) & $5,41(2)$ & $1,989 \cdot 10^{-3}(2)$ \\
\hline
\end{tabular}

(1) Valores obtenidos del modelo analítico, (2) valores obtenidos de la simulación numérica y (3) valores adoptados como dato de entrada. 


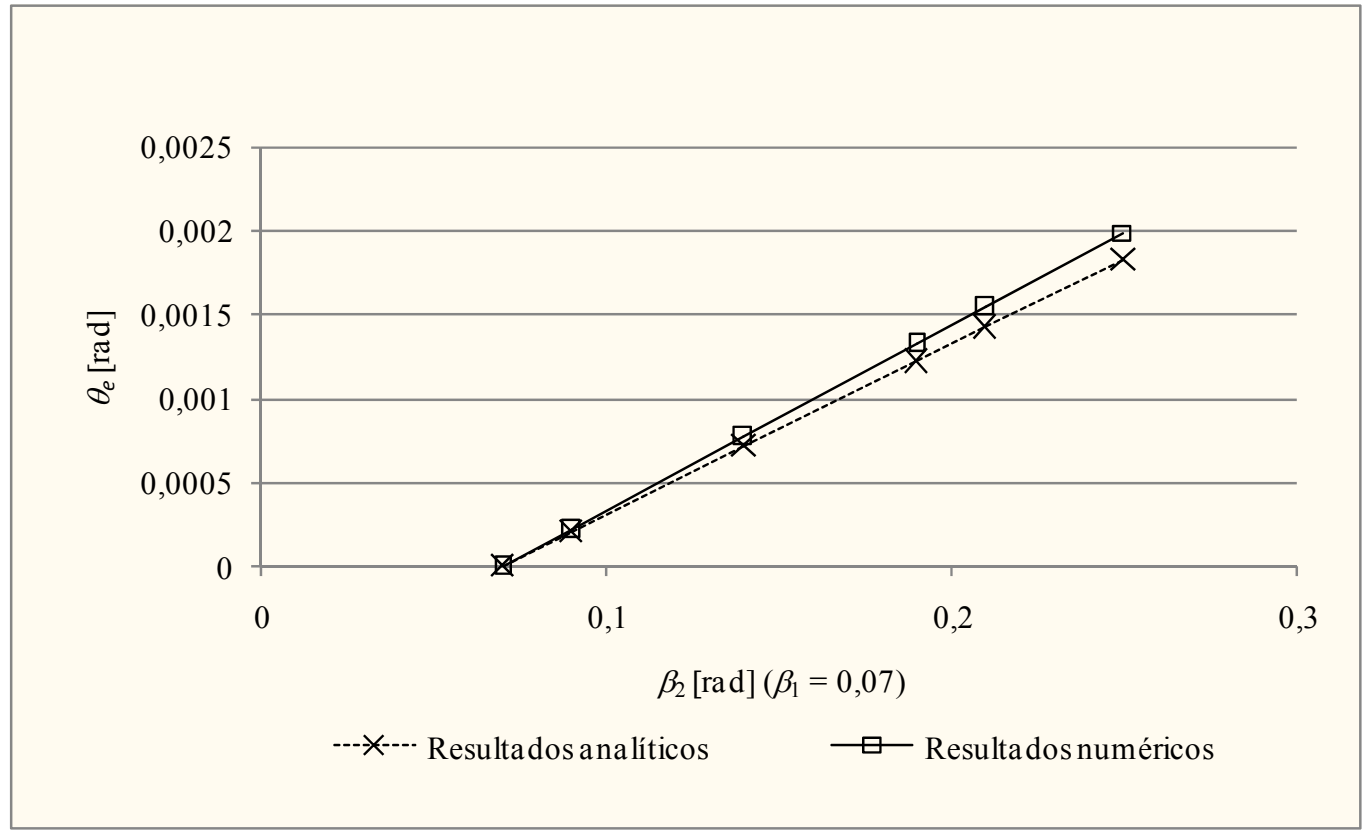

Figura 0.32 Variación del ángulo de nutación de equilibrio, $\theta_{e}$, en función de $\beta_{2}$ (con $\beta_{1}=$ 0,07 y $\left.k_{31}=0,1\right)$, para el modelo analítico y para la simulación numérica.

La Tabla 3.13 muestra para $k_{31}=0,3$ se mantiene la tendencia indicada para el caso que $k_{31}=$ 0 , donde las soluciones son muy cercanas a cero y la diferencia entre la estimación del método analítico y la simulación numérica aumenta conforme aumenta el paso de palas $\beta_{2}$ (con $\beta_{1}=$ constante), aunque aumenta la diferencia entre los valores calculados a partir del modelo analítico y la simulación numérica. Se puede observar en la Figura 3.33 que la estimación del modelo analítico es siempre menor a lo arrojado por la simulación numérica en términos del ángulo de nutación y que el valor de dicho ángulo es cercano a 0.

Tabla 0.13 Soluciones de equilibrio para distintas relaciones de paso de palas con $k_{31}=0,3$.

\begin{tabular}{|c|c|c|c|c|c|}
\hline Casos & $\begin{array}{c}\omega_{e q 1} \\
{[\mathbf{r a d} / \mathbf{s}]}\end{array}$ & $\begin{array}{c}\omega_{\text {eq2 }} \\
{[\mathbf{r a d} / \mathbf{s}]}\end{array}$ & $\begin{array}{c}\omega_{\text {eq3 }} \\
{[\mathbf{r a d} / \mathbf{s}]}\end{array}$ & $\begin{array}{c}\boldsymbol{U} \\
{[\mathbf{m} / \mathbf{s}]}\end{array}$ & $\begin{array}{c}\boldsymbol{\theta}_{\boldsymbol{e}} \\
{[\mathbf{r a d}]}\end{array}$ \\
\hline $\begin{array}{l}\beta_{1}=0,07 \\
\beta_{2}=0,07\end{array}$ & $0,0000(1)$ & $0,0000(1)$ & $292,8(3)$ & $4,57(3)$ & $0,000(1)$ \\
\hline
\end{tabular}




\begin{tabular}{|c|c|c|c|c|c|}
\hline Casos & $\begin{array}{c}\omega_{e q 1} \\
{[\mathrm{rad} / \mathrm{s}]}\end{array}$ & $\begin{array}{c}\omega_{e q 2} \\
{[\mathrm{rad} / \mathrm{s}]}\end{array}$ & $\begin{array}{c}\omega_{e q 3} \\
{[\mathrm{rad} / \mathrm{s}]}\end{array}$ & $\begin{array}{c}U \\
{[\mathbf{m} / \mathbf{s}]}\end{array}$ & $\begin{array}{c}\theta_{e} \\
{[\mathbf{r a d}]}\end{array}$ \\
\hline & $0,0000(2)$ & $0,0000(2)$ & $292,8(2)$ & 4,57 (2) & $0,000(2)$ \\
\hline \multirow{2}{*}{$\begin{array}{l}\beta_{1}=0,07 \\
\beta_{2}=0,09\end{array}$} & $0,0301(1)$ & $0,0400(1)$ & 294,0 (3) & $4,66(3)$ & $1,702 \cdot 10^{-4}(1)$ \\
\hline & $0,0325(2)$ & $0,0429(2)$ & $294,0(2)$ & $4,66(2)$ & $1,830 \cdot 10^{-4}(2)$ \\
\hline \multirow{2}{*}{$\begin{array}{l}\beta_{1}=0,07 \\
\beta_{2}=0,14\end{array}$} & $0,1064(1)$ & $0,1393(1)$ & 297,2 (3) & $4,88(3)$ & $5,898 \cdot 10^{-4}(1)$ \\
\hline & $0,1157(2)$ & $0,1501(2)$ & $297,2(2)$ & $4,88(2)$ & $6,377 \cdot 10^{-4}(2)$ \\
\hline \multirow{2}{*}{$\begin{array}{l}\beta_{1}=0,07 \\
\beta_{2}=0,19\end{array}$} & $0,1843(1)$ & $0,2372(1)$ & 300,2 (3) & $5,12(3)$ & $1,000 \cdot 10^{-3}(1)$ \\
\hline & $0,2016(2)$ & $0,2572(2)$ & $300,2(2)$ & $5,12(2)$ & $1,089 \cdot 10^{-3}(2)$ \\
\hline \multirow{2}{*}{$\begin{array}{l}\beta_{1}=0,07 \\
\beta_{2}=0,21\end{array}$} & $0,2158(1)$ & $0,2758(1)$ & $301,4(3)$ & $5,21(3)$ & $1,162 \cdot 10^{-3}(1)$ \\
\hline & 0,2367 (2) & $0,3000(2)$ & $301,4(2)$ & $5,21(2)$ & $1,268 \cdot 10^{-3}(2)$ \\
\hline \multirow{2}{*}{$\begin{array}{l}\beta_{1}=0,07 \\
\beta_{2}=0,25\end{array}$} & $0,2795(1)$ & $0,3522(1)$ & $303,7(3)$ & $5,41(3)$ & $1,481 \cdot 10^{-3}(1)$ \\
\hline & $0,3083(2)$ & $0,3852(2)$ & 303,7 (2) & $5,41(2)$ & $1,625 \cdot 10^{-3}(2)$ \\
\hline
\end{tabular}

(1) Valores obtenidos del modelo analítico, (2) valores obtenidos de la simulación numérica y (3) valores adoptados como dato de entrada. 


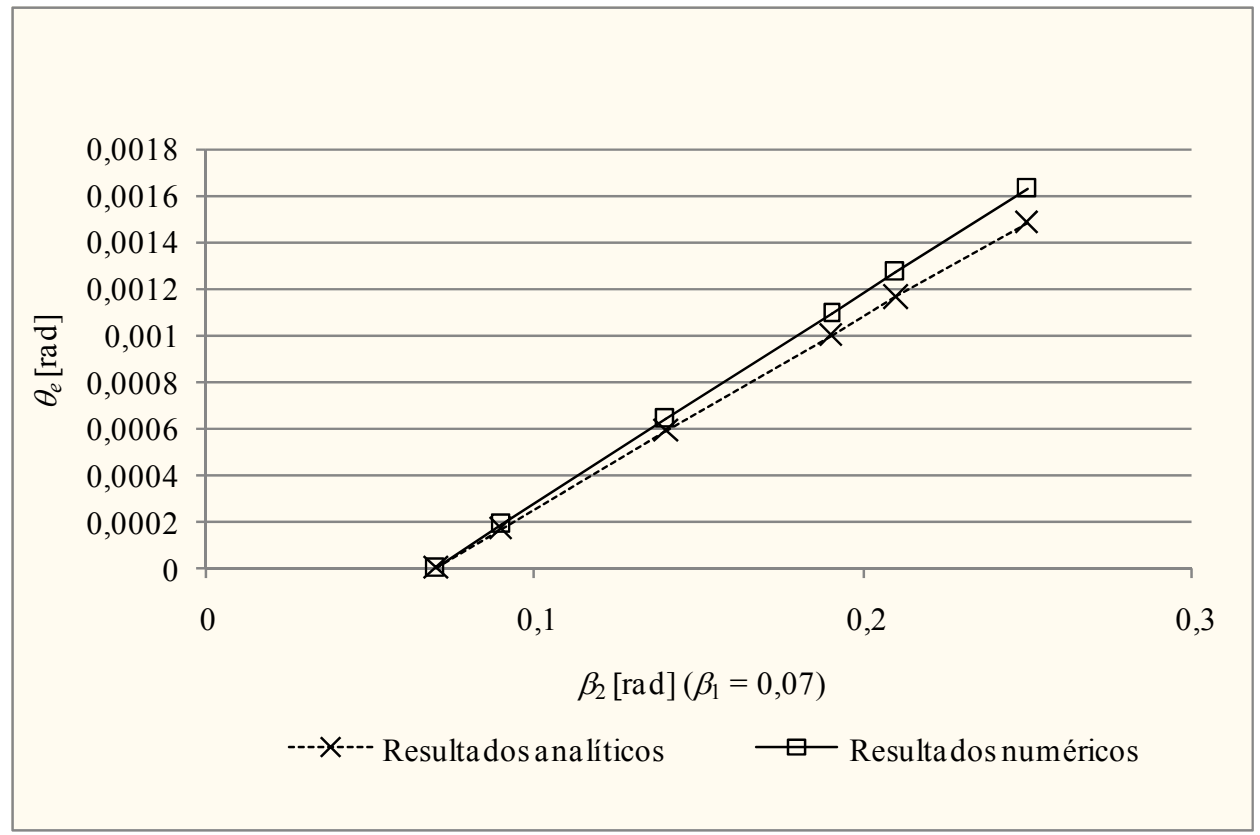

Figura 0.33 Variación del ángulo de nutación de equilibrio, $\theta_{e}$, en función de $\beta_{2}$ (con $\beta_{1}=$ 0,07 y $\left.k_{31}=0,3\right)$, para el modelo analítico y para la simulación numérica.

Los resultados para el caso $k_{31}=0,7$ se muestran en la Tabla 3.14 donde se aprecia la existencia de dos tipos de solución de equilibrio. Una de estas soluciones, para $\beta_{2}=0,09$ ( $\left.\operatorname{con} \beta_{1}=0,07\right)$ por ejemplo, se encuentra dada por un ángulo de nutación de valor nulo o muy cercano a 0 , donde las velocidades angulares $\omega_{e q 1}, \omega_{e q 2}$, son constantes de valor mucho menor que 1. Esta solución es idéntica cualitativamente a las encontradas para $k_{31}=0$. Para $\beta_{1}=0,11$ se presenta el caso límite para $k_{31}=0,7$ a partir del cual se verifica que si se produce un aumento de $k_{31}$ o un aumento de $\beta_{2}$ (con $\beta_{1}$ fijo) aparece un tipo de solución no prevista por el modelo analítico desarrollado, lo que sugiere la existencia de un punto de bifurcación de la solución, en el que el sistema deja de converger a la solución anterior (con $\theta_{\mathrm{e}}$ pequeño), y aparece otra solución que es estable, y es la que alcanza el método numérico. Este otro tipo de solución se caracteriza por ángulos de nutación grandes donde las velocidades angulares $\omega_{e q 1}$ y $\omega_{e q 2}$ tienen un comportamiento periódico asimilable con una 
función senoidal de amplitud notable para $\omega_{1}$ y $\omega_{2}$, y reducida para $\theta_{\mathrm{e}} \mathrm{y} \varphi$. Se puede ver en el caso que $\beta_{1}=0,07$ y $\beta_{2}>0,11$ los valores de la solución de equilibrio previstos por el modelo analítico resultan diferentes los que arroja la simulación numérica. En conclusión se observa que para valores de $k_{31}>0$ aparece un tipo nuevo de solución de equilibrio para el sistema, que depende principalmente del valor de este parámetro y de la diferencia de paso entre las palas, que representa una bifurcación de las soluciones previstas por el modelo analítico, como se puede observar en la Figura 3.34. Como la nueva solución es una oscilación alrededor de un nuevo valor medio, que se mantiene en el tiempo, este comportamiento podría ser el de un ciclo límite estable.

Tabla 0.14 Soluciones de equilibrio para distintas relaciones de paso de palas con $k_{31}=0,7$.

\begin{tabular}{|c|c|c|c|c|c|}
\hline Casos & $\begin{array}{c}\omega_{e q 1} \\
{[\mathrm{rad} / \mathrm{s}]}\end{array}$ & $\begin{array}{c}\omega_{e q 2} \\
{[\mathrm{rad} / \mathrm{s}]}\end{array}$ & $\begin{array}{c}\omega_{e q 3} \\
{[\mathrm{rad} / \mathrm{s}]}\end{array}$ & $\begin{array}{c}U \\
{[\mathbf{m} / \mathbf{s}]}\end{array}$ & $\begin{array}{c}\theta_{e} \\
{[\mathbf{r a d}]}\end{array}$ \\
\hline \multirow{2}{*}{$\begin{array}{l}\beta_{1}=0,07 \\
\beta_{2}=0,07\end{array}$} & $0,0000(1)$ & $0,0000(1)$ & $292,8(3)$ & $4,57(3)$ & $0,000(1)$ \\
\hline & $0,0000(2)$ & $0,0000(2)$ & $292,8(2)$ & $4,57(2)$ & $0,000(2)$ \\
\hline \multirow{2}{*}{$\begin{array}{l}\beta_{1}=0,07 \\
\beta_{2}=0,09\end{array}$} & $0,0298(1)$ & $0,0170(1)$ & 294,0 (3) & $4,66(3)$ & $1,167 \cdot 10^{-4}(1)$ \\
\hline & $0,0322(2)$ & $0,0184(2)$ & 294,0 (2) & $4,66(2)$ & $1,250 \cdot 10^{-4}(2)$ \\
\hline \multirow{2}{*}{$\begin{array}{l}\beta_{1}=0,07 \\
\beta_{2}=0,11\end{array}$} & $0,0599(1)$ & $0,0330(1)$ & $295,3(3)$ & $4,75(3)$ & $2,316 \cdot 10^{-4}(1)$ \\
\hline & $0,0650(2)$ & $0,0355(2)$ & $295,3(2)$ & $4,75(2)$ & $2,490 \cdot 10^{-4}(2)$ \\
\hline \multirow[b]{2}{*}{$\begin{array}{c}\beta_{1}=0,07 \\
\beta_{2}=0,113\end{array}$} & $0,0643(1)$ & 0,0349 (1) & $295,0(3)$ & $4,77(3)$ & $2,481 \cdot 10^{-4}(1)$ \\
\hline & $\begin{array}{c}0,0750^{*} \\
\left(3,025^{* *}\right) \\
(2)\end{array}$ & $\begin{array}{c}0,0375^{*} \\
\left(2,908^{* *}\right) \\
(2)\end{array}$ & $\begin{array}{c}295,0^{*} \\
\left(0,007^{* *}\right) \\
(2)\end{array}$ & $\begin{array}{c}4,77^{*} \\
\left(0,007^{* *}\right) \\
(2)\end{array}$ & $\begin{array}{c}0,117^{*} \\
\left(0,005^{* *}\right) \\
(2)\end{array}$ \\
\hline \multirow[b]{2}{*}{$\begin{array}{l}\beta_{1}=0,07 \\
\beta_{2}=0,12\end{array}$} & $0,0747(1)$ & $0,0396(1)$ & 294,7 (3) & $4,81(3)$ & $2,870 \cdot 10^{-4}(1)$ \\
\hline & $\begin{array}{c}0,0827^{*} \\
\left(4,017^{* *}\right) \\
(2)\end{array}$ & $\begin{array}{c}0,0400^{*} \\
\left(3,840^{* *}\right) \\
(2)\end{array}$ & $\begin{array}{c}294,7^{*} \\
\left(0,010^{* *}\right) \\
(2)\end{array}$ & $\begin{array}{c}4,81^{*} \\
\left(0,011^{* *}\right) \\
(2)\end{array}$ & $\begin{array}{c}0,156^{*} \\
\left(0,007^{* *}\right) \\
(2)\end{array}$ \\
\hline $\begin{array}{l}\beta_{1}=0,07 \\
\beta_{2}=0,13\end{array}$ & $0,0898(1)$ & $0,0463(1)$ & 295,1 (3) & $4,87(3)$ & $3,423 \cdot 10^{-4}(1)$ \\
\hline
\end{tabular}




\begin{tabular}{|c|c|c|c|c|c|}
\hline Casos & $\begin{array}{c}\omega_{e q 1} \\
{[\mathrm{rad} / \mathrm{s}]}\end{array}$ & $\begin{array}{c}\omega_{e q 2} \\
{[\mathrm{rad} / \mathrm{s}]}\end{array}$ & $\begin{array}{c}\omega_{e q 3} \\
{[\mathrm{rad} / \mathrm{s}]}\end{array}$ & $\begin{array}{c}\boldsymbol{U} \\
{[\mathbf{m} / \mathbf{s}]}\end{array}$ & $\begin{array}{c}\theta_{e} \\
{[\mathbf{r a d}]}\end{array}$ \\
\hline & $\begin{array}{c}0,1000^{*} \\
\left(5,120^{* *}\right) \\
(2)\end{array}$ & $\begin{array}{c}0,0534^{*} \\
\left(4,917^{* *}\right) \\
(2) \\
\end{array}$ & $\begin{array}{c}295,1^{*} \\
\left(0,019^{* *}\right) \\
(2)\end{array}$ & $\begin{array}{c}4,87^{*} \\
\left(0,021^{* *}\right) \\
(2) \\
\end{array}$ & $\begin{array}{c}0,199 * \\
(0,009 * *) \\
(2) \\
\end{array}$ \\
\hline \multirow[b]{2}{*}{$\begin{array}{l}\beta_{1}=0,07 \\
\beta_{2}=0,14\end{array}$} & $0,1048(1)$ & $0,0524(1)$ & $295,2(3)$ & 4,93 (3) & $3,968 \cdot 10^{-4}(1)$ \\
\hline & $\begin{array}{c}0,1000^{*} \\
\left(6,100^{* *}\right) \\
(2)\end{array}$ & $\begin{array}{c}0,0650^{*} \\
\left(5,825^{* *}\right) \\
(2) \\
\end{array}$ & $\begin{array}{c}295,2^{*} \\
\left(0,073^{* *}\right) \\
(2)\end{array}$ & $\begin{array}{c}4,93^{*} \\
\left(0,0315^{* *}\right) \\
(2)\end{array}$ & $\begin{array}{c}0,236^{*} \\
\left(0,010^{* *}\right) \\
(2)\end{array}$ \\
\hline \multirow[b]{2}{*}{$\begin{array}{l}\beta_{1}=0,07 \\
\beta_{2}=0,19\end{array}$} & $0,1793(1)$ & $0,0753(1)$ & $295,0(3)$ & $5,21(3)$ & $6,593 \cdot 10^{-4}(1)$ \\
\hline & $\begin{array}{c}0,2250^{*} \\
\left(9,875^{* *}\right) \\
(2)\end{array}$ & $\begin{array}{c}0,1075^{*} \\
\left(9,463^{* *}\right) \\
(2)\end{array}$ & $\begin{array}{c}295,0^{*} \\
\left(0,075^{* *}\right) \\
(2)\end{array}$ & $\begin{array}{c}5,21^{*} \\
\left(0,081^{* *}\right) \\
(2)\end{array}$ & $\begin{array}{c}0,385^{*} \\
\left(0,017^{* *}\right) \\
(2)\end{array}$ \\
\hline \multirow[b]{2}{*}{$\begin{array}{l}\beta_{1}=0,07 \\
\beta_{2}=0,21\end{array}$} & 0,2089 (1) & 0,0809 (1) & 294,7 (3) & $5,33(3)$ & $7,601 \cdot 10^{-4}(1)$ \\
\hline & $\begin{array}{c}0,2500^{*} \\
\left(11,150^{* *}\right) \\
(2) \\
\end{array}$ & $\begin{array}{c}0,1200^{*} \\
\left(10,710^{* *}\right) \\
(2)\end{array}$ & $\begin{array}{c}294,7^{*} \\
\left(0,095^{* *}\right) \\
(2)\end{array}$ & $\begin{array}{c}5,33^{*} \\
\left(0,105^{* *}\right) \\
(2) \\
\end{array}$ & $\begin{array}{c}0,437^{*} \\
\left(0,019^{* *}\right) \\
(2)\end{array}$ \\
\hline \multirow[b]{2}{*}{$\begin{array}{l}\beta_{1}=0,07 \\
\beta_{2}=0,25\end{array}$} & $0,2677(1)$ & $0,0863(1)$ & $293,9(3)$ & $5,55(3)$ & $9,569 \cdot 10^{-4}(1)$ \\
\hline & $\begin{array}{c}0,3750^{*} \\
\left(13,730^{* *}\right) \\
(2) \\
\end{array}$ & $\begin{array}{c}0,1750^{*} \\
\left(13,125^{* *}\right) \\
(2) \\
\end{array}$ & $\begin{array}{c}293,9 * \\
(0,144 * *) \\
(2)\end{array}$ & $\begin{array}{c}5,55^{*} \\
\left(0,156^{* *}\right) \\
(2)\end{array}$ & $\begin{array}{c}0,539^{*} \\
(0,024 * *) \\
(2) \\
\end{array}$ \\
\hline
\end{tabular}

(1) Valores obtenidos del modelo analítico, (2) valores obtenidos de la simulación numérica y (3) valores adoptados como dato de entrada.

*Función senoidal con valor medio indicado.

** Función senoidal con valor de amplitud indicada. 


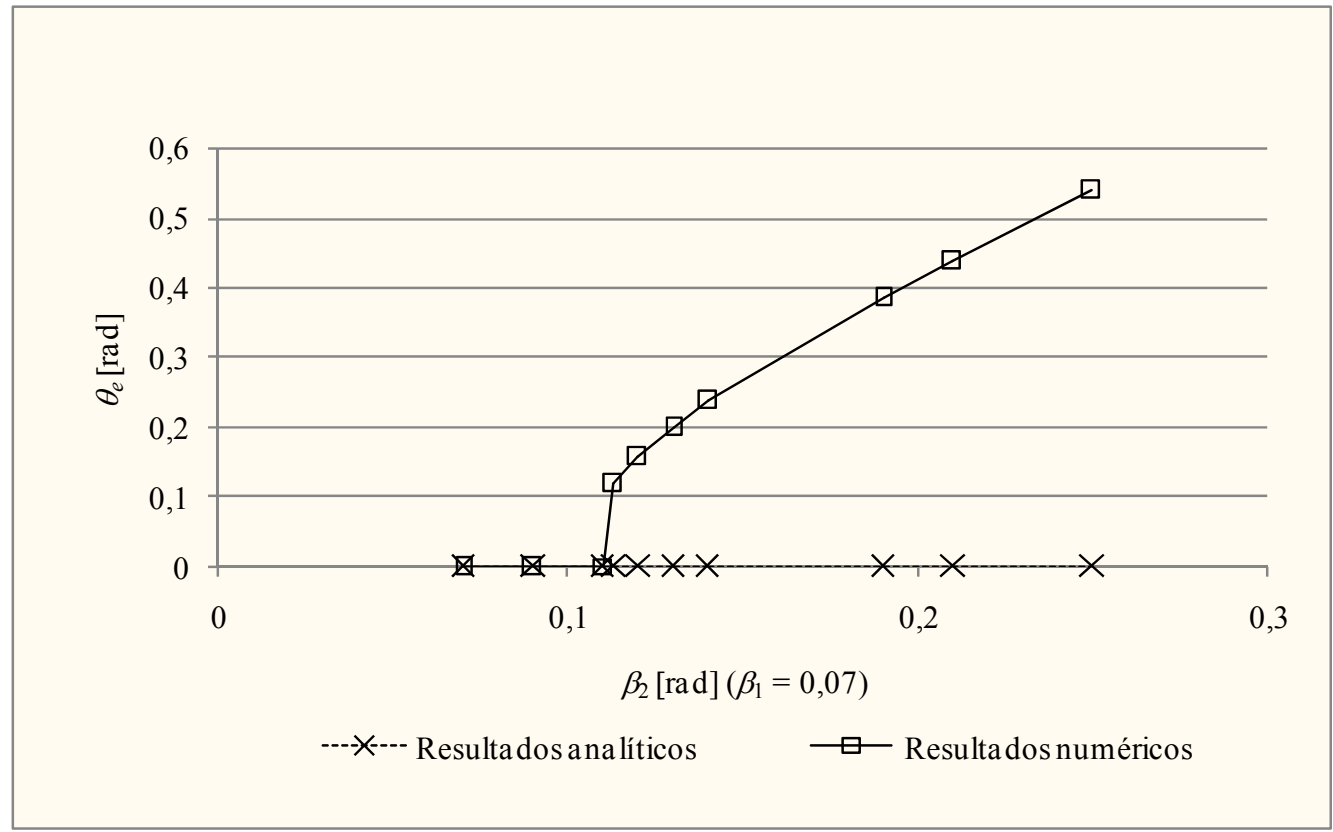

Figura 0.34 Diagrama de bifurcación para el caso $\beta_{1}=0,07$ y $k_{31}=0,7$. Variación del ángulo de nutación de equilibrio, $\theta_{e}$, en función de $\beta_{2}$ para el modelo analítico y para la simulación numérica. Para la solución bifurcada $\theta_{e}$ se presenta el valor medio de $\theta$ en un ciclo de variación.

Se comprueba que si $k_{31}=1$, a un muy pequeño valor de $\delta_{\beta}$ aparece la bifurcación de las soluciones de la simulación numérica al respecto de las soluciones analíticas. Vale decir que la sensibilidad del sistema al parámetro $\delta_{\beta}$ en la introducción de una solución de este tipo depende del alejamiento del centro de masas al respecto del plano que contiene a las palas (dada por el valor del parámetro $k_{31}$ ).

La Tabla 3.15 muestra otra vez para $k_{31}=1$ la existencia de dos tipos de solución de equilibrio. Una de estas soluciones, para $\delta_{\beta}=0\left(\beta_{1}=0,07, \beta_{2}=0,07\right)$, se encuentra dada por un ángulo de nutación de valor nulo o muy cercano a 0 , donde las velocidades angulares $\omega_{e q 1}$ y $\omega_{e q 2}$ son constantes de valor mucho menor que 1 (Tipo I). Esta solución es idéntica cualitativamente a las encontradas para $k_{31}=0$. Un aumento pequeño de $\delta_{\beta}$ da lugar enseguida 
a la aparición de la bifurcación. Este otro tipo de solución que se presenta en la simulación numérica cuando aumenta $\delta_{\beta}$, está dada por ángulos de nutación del orden de la unidad donde las velocidades angulares $\omega_{e q 1}, \omega_{e q 2}$ pasan de ser constantes a presentar un comportamiento periódico asimilable con una función senoidal de amplitud considerable (Tipo II). Por otro lado también se verifica que el ángulo de rotación propia $\varphi$ mantiene un ritmo de crecimiento constante aproximadamente igual a la velocidad de rotación $\omega_{3}, \mathrm{y}$ un ángulo de precesión $\psi$ muy pequeño. También se puede comprobar que el ángulo de nutación aumenta al aumentar $\beta_{2}$ (con $\beta_{1}$ constante) en la Figura 3.35.

Tabla 0.15 Soluciones de equilibrio para distintas relaciones de paso de palas con $k_{31}=1$.

\begin{tabular}{|c|c|c|c|c|c|}
\hline Casos & $\begin{array}{c}\omega_{e q 1} \\
{[\mathrm{rad} / \mathrm{s}]}\end{array}$ & $\begin{array}{c}\omega_{e q 2} \\
{[\mathrm{rad} / \mathrm{s}]}\end{array}$ & $\begin{array}{c}\omega_{e q 3} \\
{[\mathbf{r a d} / \mathbf{s}]}\end{array}$ & $\begin{array}{c}U \\
{[\mathbf{m} / \mathbf{s}]}\end{array}$ & $\begin{array}{c}\theta_{e} \\
{[\mathbf{r a d}]}\end{array}$ \\
\hline \multirow{2}{*}{$\begin{array}{l}\beta_{1}=0,07 \\
\beta_{2}=0,07\end{array}$} & $0,0000(1)$ & $0,0000(1)$ & $292,8(3)$ & $4,57(3)$ & $0,000(1)$ \\
\hline & $0,0000(2)$ & $0,0000(2)$ & $292,8(2)$ & $4,57(2)$ & $0,000(2)$ \\
\hline \multirow[b]{2}{*}{$\begin{array}{l}\beta_{1}=0,07 \\
\beta_{2}=0,08\end{array}$} & $0,0141(1)$ & $-0,0031(1)$ & $280,9(3)$ & $5,04(3)$ & $5,148 \cdot 10^{-5}(1)$ \\
\hline & $\begin{array}{c}0,0675^{*} \\
\left(24,918^{* *}\right) \\
(2) \\
\end{array}$ & $\begin{array}{c}0,0000^{*} \\
\left(23,800^{* *}\right) \\
(2) \\
\end{array}$ & $\begin{array}{c}280,9 * \\
\left(0,355^{* *}\right) \\
(2)\end{array}$ & $\begin{array}{c}5,04^{*} \\
\left(0,196^{* *}\right) \\
(2) \\
\end{array}$ & $\begin{array}{c}0,745^{*} \\
(0,043 * *) \\
(2) \\
\end{array}$ \\
\hline \multirow[b]{2}{*}{$\begin{array}{l}\beta_{1}=0,07 \\
\beta_{2}=0,09\end{array}$} & $0,0257(1)$ & $-0,0091(1)$ & $256,1(3)$ & $4,90(3)$ & $1,065 \cdot 10^{-4}(1)$ \\
\hline & $\begin{array}{c}0,0500 * \\
(25,360 * *) \\
(2)\end{array}$ & $\begin{array}{c}0,0025^{*} \\
\left(24,218^{* *}\right) \\
(2)\end{array}$ & $\begin{array}{c}256,1 * \\
\left(0,405^{* *}\right) \\
(2)\end{array}$ & $\begin{array}{c}4,90 * \\
(0,384 * *) \\
(2)\end{array}$ & $\begin{array}{c}0,757 * \\
(0,044 * *) \\
(2)\end{array}$ \\
\hline \multirow[b]{2}{*}{$\begin{array}{l}\beta_{1}=0,07 \\
\beta_{2}=0,14\end{array}$} & 0,0991 (1) & $-0,0234(1)$ & $281,5(3)$ & $5,09(3)$ & $3,616 \cdot 10^{-4}(1)$ \\
\hline & $\begin{array}{c}0,1750^{*} \\
\left(27,175^{* *}\right) \\
(2) \\
\end{array}$ & $\begin{array}{c}0,0100^{*} \\
\left(25,940^{* *}\right) \\
(2) \\
\end{array}$ & $\begin{array}{c}281,5^{*} \\
\left(0,436^{* *}\right) \\
(2)\end{array}$ & $\begin{array}{c}5,09 * \\
\left(0,433^{* *}\right) \\
(2) \\
\end{array}$ & $\begin{array}{c}0,815^{*} \\
\left(0,047^{* *}\right) \\
(2)\end{array}$ \\
\hline \multirow[b]{2}{*}{$\begin{array}{l}\beta_{1}=0,07 \\
\beta_{2}=0,21\end{array}$} & 0,1977 (1) & $-0,0661(1)$ & $281,2(3)$ & $5,33(3)$ & $7,414 \cdot 10^{-4}(1)$ \\
\hline & $\begin{array}{c}0,4000^{*} \\
(29,600 * *) \\
(2)\end{array}$ & $\begin{array}{c}0,0000^{*} \\
\left(28,200^{* *}\right) \\
(2)\end{array}$ & $\begin{array}{c}281,2^{*} \\
\left(0,523^{* *}\right) \\
(2)\end{array}$ & $\begin{array}{c}5,33^{*} \\
\left(0,505^{* *}\right) \\
(2)\end{array}$ & $\begin{array}{c}0,896 \\
\left(0,051^{* *}\right) \\
(2)\end{array}$ \\
\hline
\end{tabular}


(1) Valores obtenidos del modelo analítico, (2) valores obtenidos de la simulación numérica y (3) valores adoptados como dato de entrada.

*Función tipo senoidal con valor medio indicado.

** Función tipo senoidal con valor de amplitud indicada.

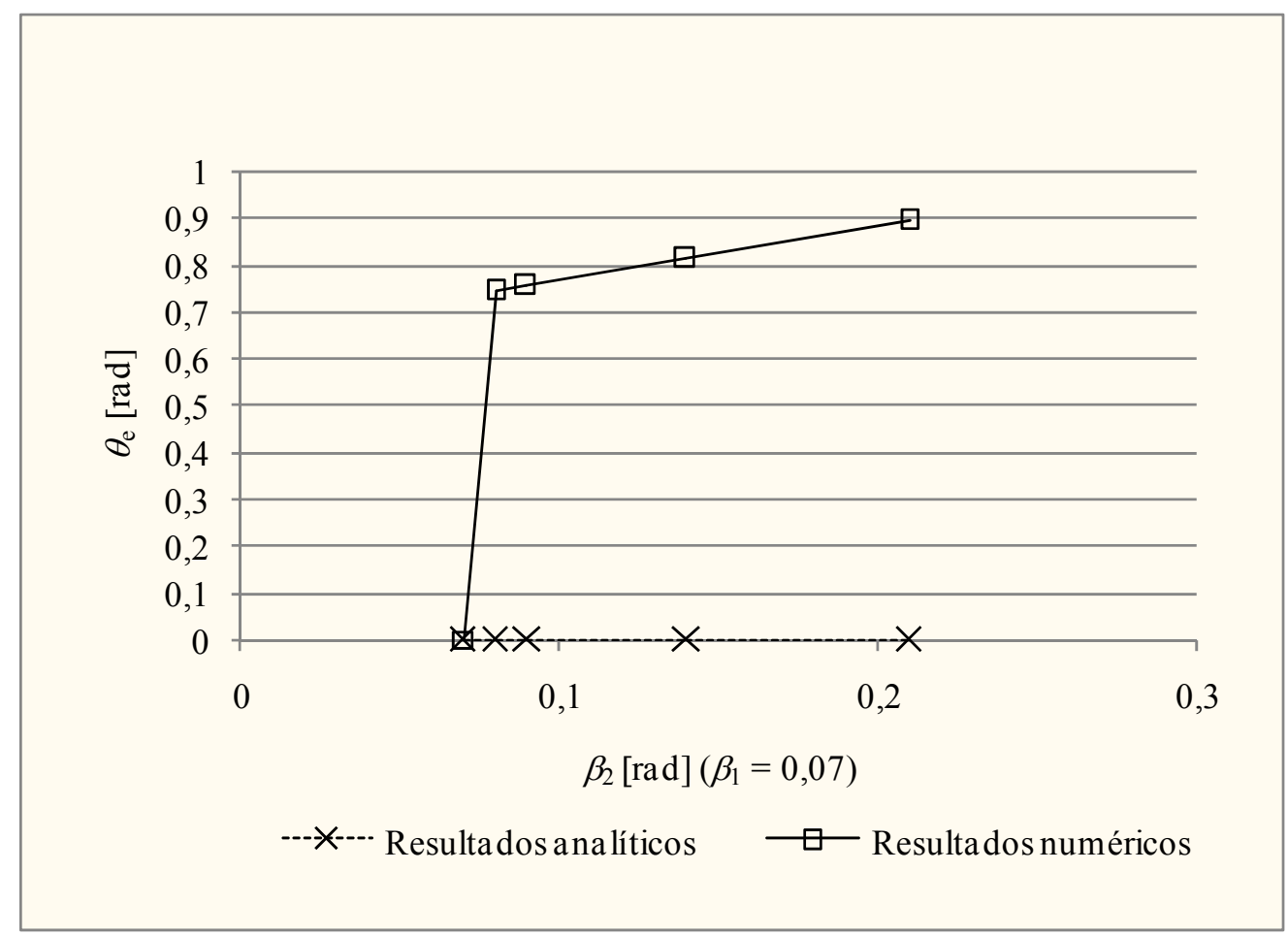

Figura 0.35 Diagrama de bifurcación para el caso $\beta_{1}=0,07$ y $k_{31}=1$. Variación del ángulo de nutación de equilibrio, $\theta_{e}$, en función de $\beta_{2}$ para el modelo analítico y para la simulación numérica. Para la solución bifurcada $\theta_{e}$ representa el valor medio de $\theta$ en un ciclo de variación.

La Tabla 3.16 y la Tabla 3.17 muestran que para $k_{31}=-0,3$ y para $k_{31}=-0,7$ sólo se presenta el tipo de solución Tipo I. También se observa que el modelo analítico arroja predicciones de valores ligeramente mayores a los de las simulaciones numéricas, como se verifica en la Figura 3.36 y en la Figura 3.37. 
Tabla 0.16 Soluciones de equilibrio para distintas relaciones de paso de palas con $k_{31}=-0,3$.

\begin{tabular}{|c|c|c|c|c|c|}
\hline Casos & $\begin{array}{c}\omega_{e q 1} \\
{[\mathrm{rad} / \mathrm{s}]}\end{array}$ & $\begin{array}{c}\omega_{e q 2} \\
{[\mathrm{rad} / \mathrm{s}]}\end{array}$ & $\begin{array}{c}\omega_{e q 3} \\
{[\mathrm{rad} / \mathrm{s}]}\end{array}$ & $\begin{array}{c}U \\
{[\mathbf{m} / \mathbf{s}]}\end{array}$ & $\begin{array}{c}\theta_{e} \\
{[\mathbf{r a d}]}\end{array}$ \\
\hline \multirow{2}{*}{$\begin{array}{l}\beta_{1}=0,07 \\
\beta_{2}=0,07\end{array}$} & $0,0000(1)$ & $0,0000(1)$ & $292,8(3)$ & 4,57 (3) & $0,000(1)$ \\
\hline & $0,0000(2)$ & $0,0000(2)$ & $292,8(2)$ & 4,57 (2) & $2,107 \cdot 10^{-8}(2)$ \\
\hline \multirow{2}{*}{$\begin{array}{l}\beta_{1}=0,07 \\
\beta_{2}=0,09\end{array}$} & 0,0305 (1) & $0,0770(1)$ & 294,0 (3) & $4,66(3)$ & $2,818 \cdot 10^{-4}(1)$ \\
\hline & $0,0330(2)$ & $0,0810(2)$ & $294,0(2)$ & $4,66(2)$ & $2,976 \cdot 10^{-4}(2)$ \\
\hline \multirow{2}{*}{$\begin{array}{l}\beta_{1}=0,07 \\
\beta_{2}=0,14\end{array}$} & 0,1079 (1) & 0,2749 (1) & 297,2 (3) & $4,88(3)$ & $9,937 \cdot 10^{-4}(1)$ \\
\hline & $0,1174(2)$ & 0,2908 (2) & 297,2 (2) & $4,88(2)$ & $1,055 \cdot 10^{-3}(2)$ \\
\hline \multirow{2}{*}{$\begin{array}{l}\beta_{1}=0,07 \\
\beta_{2}=0,19\end{array}$} & 0,1870 (1) & $0,4802(1)$ & 300,2 (3) & $5,11(3)$ & $1,717 \cdot 10^{-3}(1)$ \\
\hline & 0,2047 (2) & $0,2112(2)$ & $300,2(2)$ & $5,11(2)$ & $1,834 \cdot 10^{-3}(2)$ \\
\hline \multirow{2}{*}{$\begin{array}{l}\beta_{1}=0,07 \\
\beta_{2}=0,21\end{array}$} & 0,2190 (1) & $0,5645(1)$ & $301,4(3)$ & $5,21(3)$ & $2,009 \cdot 10^{-3}(1)$ \\
\hline & 0,2404 (2) & $0,6025(2)$ & $301,4(2)$ & $5,21(2)$ & $2,152 \cdot 10^{-3}(2)$ \\
\hline \multirow{2}{*}{$\begin{array}{l}\beta_{1}=0,07 \\
\beta_{2}=0,25\end{array}$} & 0,2838 (1) & 0,7368 (1) & 303,7 (3) & $5,40(3)$ & $2,600 \cdot 10^{-3}(1)$ \\
\hline & $0,3132(2)$ & $0,7906(2)$ & 303,7 (2) & $5,40(2)$ & $2,800 \cdot 10^{-3}(2)$ \\
\hline
\end{tabular}

(1) Valores obtenidos del modelo analítico, (2) valores obtenidos de la simulación numérica y (3) valores adoptados como dato de entrada. 


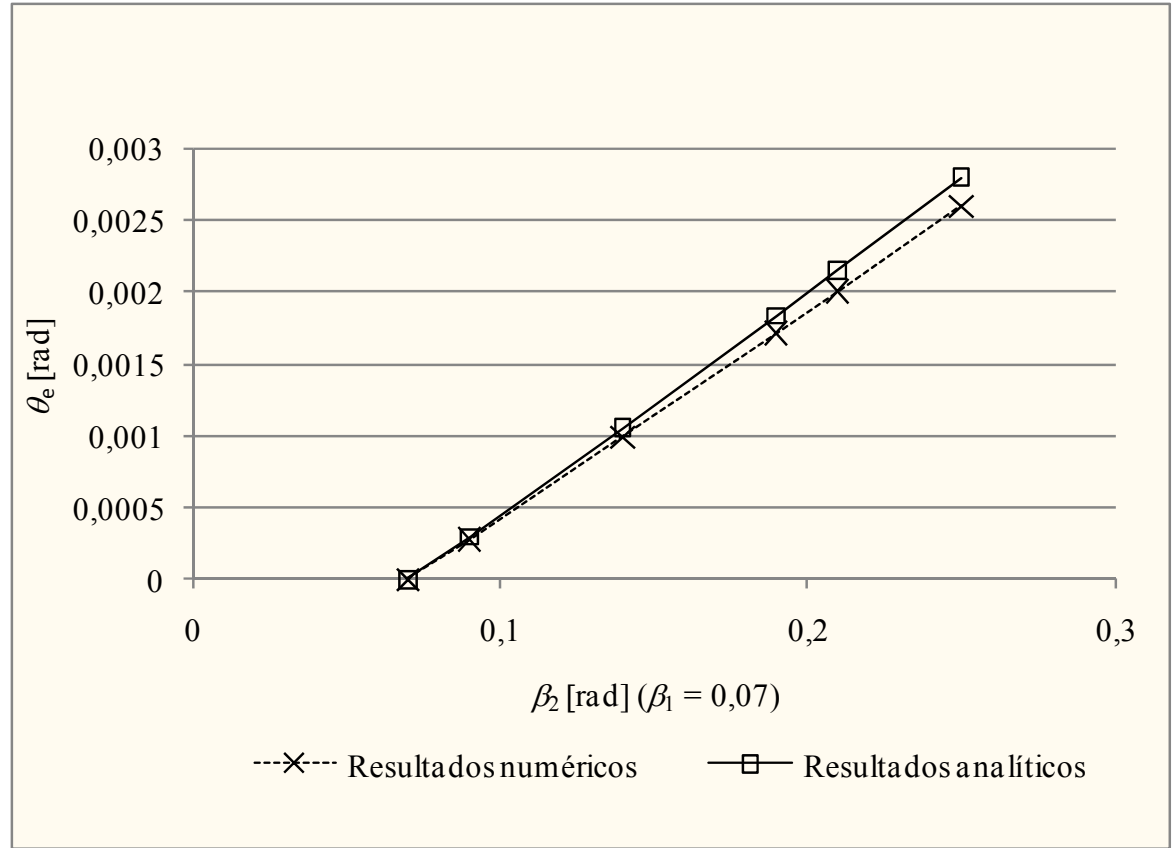

Figura 0.36 Variación del ángulo de nutación de equilibrio, $\theta_{\mathrm{e}}$, en función de $\beta_{2}$ (con $\beta_{1}=$ 0,07 y $\left.k_{31}=-0,3\right)$, para el modelo analítico y para la simulación numérica.

Tabla 0.17 Soluciones de equilibrio para distintas relaciones de paso de palas con $k_{31}=-0,7$.

\begin{tabular}{|c|c|c|c|c|c|}
\hline Casos & $\begin{array}{c}\omega_{e q 1} \\
{[\mathrm{rad} / \mathrm{s}]}\end{array}$ & $\begin{array}{c}\omega_{e q 2} \\
{[\mathrm{rad} / \mathrm{s}]}\end{array}$ & $\begin{array}{c}\omega_{e q 3} \\
{[\mathrm{rad} / \mathrm{s}]}\end{array}$ & $\begin{array}{c}U \\
{[\mathbf{m} / \mathbf{s}]}\end{array}$ & $\begin{array}{c}\theta_{e} \\
{[\mathbf{r a d}]}\end{array}$ \\
\hline \multirow{2}{*}{$\begin{array}{l}\beta_{1}=0,07 \\
\beta_{2}=0,07\end{array}$} & $0,0000(1)$ & $0,0000(1)$ & $292,8(3)$ & $4,57(3)$ & $0,000(1)$ \\
\hline & $0,0000(2)$ & $0,0000(2)$ & $292,8(2)$ & $4,57(2)$ & $0,000(2)$ \\
\hline \multirow{2}{*}{$\begin{array}{l}\beta_{1}=0,07 \\
\beta_{2}=0,09\end{array}$} & $0,0308(1)$ & $0,1036(1)$ & $294,0(3)$ & $4,66(3)$ & $3,675 \cdot 10^{-4}(1)$ \\
\hline & $0,0334(2)$ & $0,1073(2)$ & $294,0(2)$ & $4,66(2)$ & $3,821 \cdot 10^{-4}(2)$ \\
\hline \multirow{2}{*}{$\begin{array}{l}\beta_{1}=0,07 \\
\beta_{2}=0,14\end{array}$} & $0,1090(1)$ & $0,3721(1)$ & $297,2(3)$ & $4,88(3)$ & $1,305 \cdot 10^{-3}(1)$ \\
\hline & $0,1189(2)$ & $0,3875(2)$ & $297,2(2)$ & $4,88(2)$ & $1,364 \cdot 10^{-3}(2)$ \\
\hline $\begin{array}{l}\beta_{1}=0,07 \\
\beta_{2}=0,19\end{array}$ & $0,1889(1)$ & $0,6546(1)$ & $300,2(3)$ & $5,11(3)$ & $2,270 \cdot 10^{-3}(1)$ \\
\hline
\end{tabular}




\begin{tabular}{|c|c|c|c|c|c|}
\hline Casos & $\begin{array}{c}\boldsymbol{\omega}_{\text {eq1 }} \\
{[\mathbf{r a d} / \mathbf{s}]}\end{array}$ & $\begin{array}{c}\boldsymbol{\omega}_{\text {eq2 }} \\
{[\mathbf{r a d} / \mathbf{s}]}\end{array}$ & $\begin{array}{c}\boldsymbol{\omega}_{\text {eq3 }} \\
{[\mathbf{r a d} / \mathbf{s}]}\end{array}$ & $\begin{array}{c}\boldsymbol{U} \\
{[\mathbf{m} / \mathbf{s}]}\end{array}$ & $\begin{array}{c}\boldsymbol{\theta}_{\boldsymbol{e}} \\
{[\mathbf{r a d}]}\end{array}$ \\
\hline & $0,2073(2)$ & $0,6859(2)$ & $300,2(2)$ & $5,11(2)$ & $2,387 \cdot 10^{-3}(2)$ \\
\hline $\begin{array}{l}\beta_{1}=0,07 \\
\beta_{2}=0,21\end{array}$ & $0,2213(1)$ & $0,7717(1)$ & $301,4(3)$ & $5,21(3)$ & $2,664 \cdot 10^{-3}(1)$ \\
\hline$\beta_{1}=0,07$ & $0,2435(2)$ & $0,8107(2)$ & $301,4(2)$ & $5,21(2)$ & $2,809 \cdot 10^{-3}(2)$ \\
$\beta_{2}=0,25$ & $0,3173(2)$ & $1,0697(2)$ & $303,7(2)$ & $5,40(2)$ & $3,674 \cdot 10^{-3}(2)$ \\
\hline
\end{tabular}

(1) Valores obtenidos del modelo analítico, (2) valores obtenidos de la simulación numérica y (3) valores adoptados como dato de entrada.

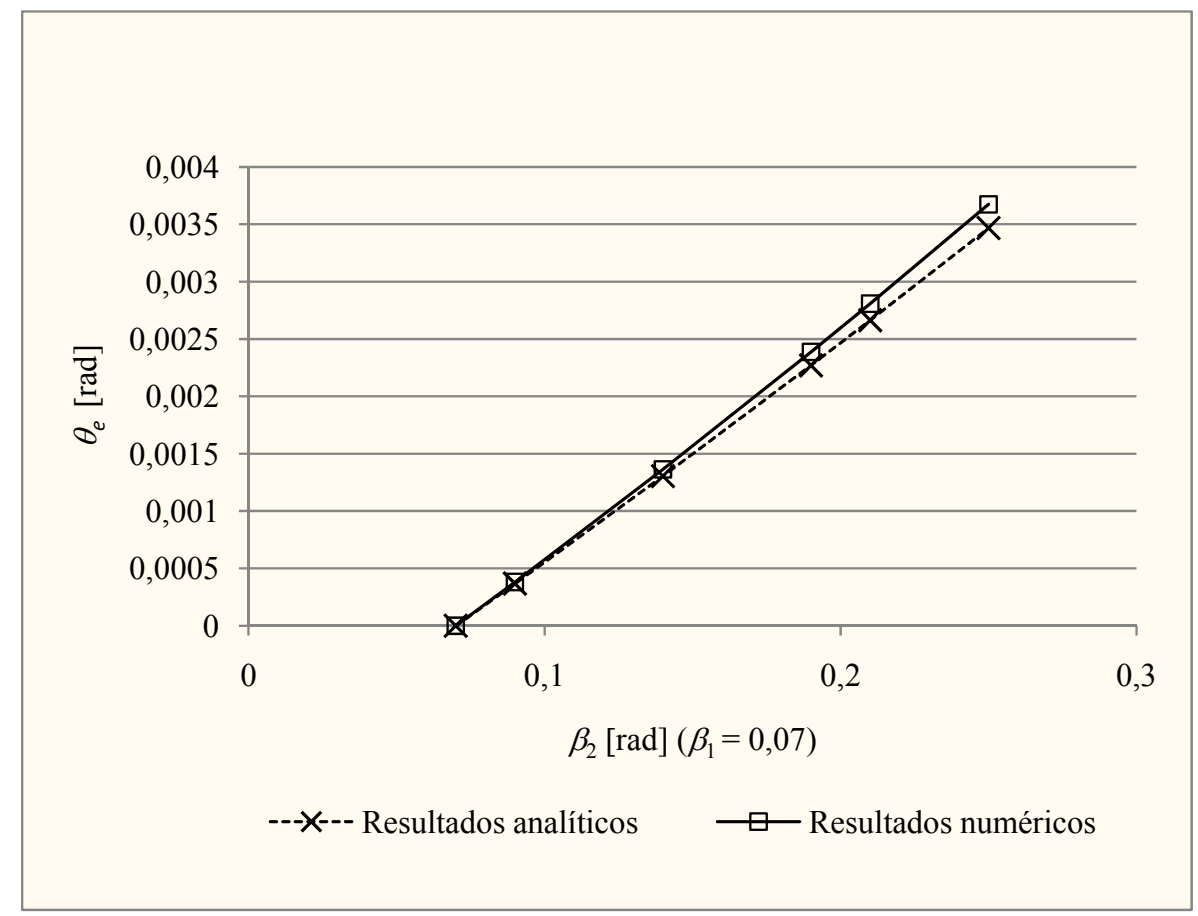

Figura 0.37 Variación del ángulo de nutación de equilibrio, $\theta_{e}$, en función de $\beta_{2}$ (con $\beta_{1}=$ 0,07 y $\left.k_{31}=-0,7\right)$, para el modelo analítico y para la simulación numérica. 
La Tabla 3.18 muestra que, para $k_{31}=-1$, nuevamente, la existencia de los dos tipos de solución de equilibrio mencionadas anteriormente. A partir de $\beta_{2}=0,25\left(\beta_{1}=0,07\right)$ se verifica que un aumento de $k_{31}$ o un aumento de $\delta_{\beta}=0$ da lugar a una solución Tipo II.

El diagrama de bifurcación para el caso que $\beta_{1}=0,07$ y $k_{31}=-1$ se presenta en la Figura 3.38.

Tabla 0.18 Soluciones de equilibrio para distintas relaciones de paso de palas con $k_{31}=-1$.

\begin{tabular}{|c|c|c|c|c|c|}
\hline Casos & $\begin{array}{c}\omega_{e q 1} \\
{[\mathrm{rad} / \mathrm{s}]}\end{array}$ & $\begin{array}{c}\omega_{e q 2} \\
{[\mathrm{rad} / \mathrm{s}]}\end{array}$ & $\begin{array}{c}\omega_{e q 3} \\
{[\mathrm{rad} / \mathrm{s}]}\end{array}$ & $\begin{array}{c}U \\
{[\mathbf{m} / \mathbf{s}]}\end{array}$ & $\begin{array}{c}\theta_{e} \\
{[\mathbf{r a d}]}\end{array}$ \\
\hline \multirow{2}{*}{$\begin{array}{l}\beta_{1}=0,07 \\
\beta_{2}=0,07\end{array}$} & 0,0000 (1) & $0,0000(1)$ & $292,8(3)$ & $4,57(3)$ & $0,000(1)$ \\
\hline & $0,0000(2)$ & $0,0000(2)$ & $292,8(2)$ & $4,57(2)$ & $0,000(2)$ \\
\hline \multirow{2}{*}{$\begin{array}{l}\beta_{1}=0,07 \\
\beta_{2}=0,09\end{array}$} & $0,0310(1)$ & $0,1246(1)$ & 294,0 (3) & $4,66(3)$ & $4,365 \cdot 10^{-4}(1)$ \\
\hline & $0,0338(2)$ & $0,1274(2)$ & $294,0(2)$ & $4,66(2)$ & $4,481 \cdot 10^{-4}(2)$ \\
\hline \multirow{2}{*}{$\begin{array}{l}\beta_{1}=0,07 \\
\beta_{2}=0,14\end{array}$} & 0,1099 (1) & 0,4489 (1) & 297,2 (3) & $4,88(3)$ & $1,555 \cdot 10^{-3}(1)$ \\
\hline & $0,1202(2)$ & $0,4616(2)$ & $297,2(2)$ & $4,88(2)$ & $1,605 \cdot 10^{-3}(2)$ \\
\hline \multirow{2}{*}{$\begin{array}{l}\beta_{1}=0,07 \\
\beta_{2}=0,19\end{array}$} & 0,1905 (1) & $0,7930(1)$ & 300,2 (3) & $5,12(3)$ & $2,717 \cdot 10^{-3}(1)$ \\
\hline & 0,2097 (2) & 0,8197 (2) & $300,2(2)$ & $5,12(2)$ & $2,819 \cdot 10^{-3}(2)$ \\
\hline \multirow{2}{*}{$\begin{array}{l}\beta_{1}=0,07 \\
\beta_{2}=0,21\end{array}$} & $0,2231(1)$ & 0,9355 (1) & $301,4(3)$ & $5,21(3)$ & $3,191 \cdot 10^{-3}(1)$ \\
\hline & 0,2463 (2) & $0,9701(2)$ & $301,4(2)$ & $5,21(2)$ & $3,321 \cdot 10^{-3}(2)$ \\
\hline \multirow{2}{*}{$\begin{array}{l}\beta_{1}=0,07 \\
\beta_{2}=0,25\end{array}$} & 0,2893 (1) & $1,2314(1)$ & $303,6(3)$ & $5,40(3)$ & $4,166 \cdot 10^{-3}(1)$ \\
\hline & $0,3211(2)$ & $1,2835(2)$ & $303,6(2)$ & $5,40(2)$ & $4,357 \cdot 10^{-3}(2)$ \\
\hline \multirow{3}{*}{$\begin{array}{l}\beta_{1}=0,07 \\
\beta_{2}=0,30\end{array}$} & $0,7236(1)$ & $3,5522(1)$ & 304,9 (3) & $6,18(3)$ & $1,189 \cdot 10^{-2}(1)$ \\
\hline & $0,4300 *$ & $1,7175^{*}$ & $304,9 *$ & $6,18^{*}$ & $0,161 *$ \\
\hline & $\begin{array}{c}2,404^{* *} \\
(2)\end{array}$ & $\begin{array}{c}2,315^{* *} \\
(2)\end{array}$ & $\begin{array}{c}0,031^{* *} \\
(2)\end{array}$ & $\begin{array}{c}0,484 * * \\
(2)\end{array}$ & $\begin{array}{c}8,350 \cdot 10^{-3} * \\
(2)\end{array}$ \\
\hline
\end{tabular}

(1) Valores obtenidos del modelo analítico, (2) valores obtenidos de la simulación numérica y (3) valores adoptados como dato de entrada.

*Función tipo senoidal con valor medio indicado. 
** Función tipo senoidal con valor de amplitud indicada.

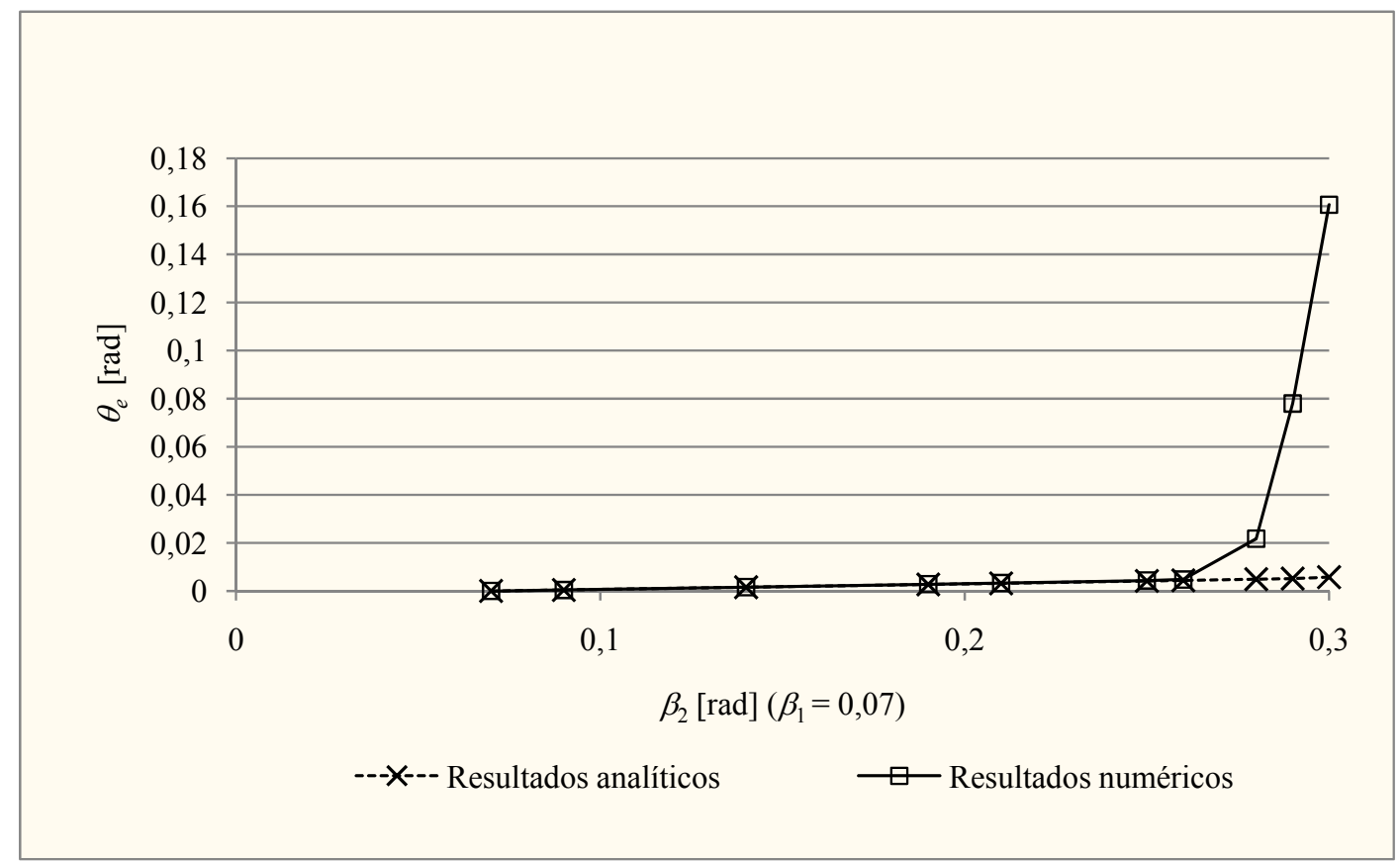

Figura 0.38 Variación del ángulo de nutación de equilibrio, $\theta_{e}$, en función de $\beta_{2}$ para el modelo analítico y para la simulación numérica. Para la solución bifurcada $\theta_{e}$ representa el valor medio de $\theta$ en un ciclo de variación.

Como resumen, en la Figura 3.39 se presenta el ángulo de nutación de equilibrio para distintos valores del parámetro $k_{31}$ en función de $\beta_{2}$ (con $\left.\beta_{1}=0,07\right)$. Puede observarse que el ángulo de nutación aumenta con el aumento de $k_{31}$. En la figura mencionada sólo se grafican las soluciones de equilibrio Tipo I. Puede verse que para $k_{31}=0,7$ la solución muestra una bifurcación.

Si se grafica el error relativo entre el ángulo de nutación de equilibrio de la solución del modelo analítico y la simulación numérica (Figura 3.40) en función de $\beta_{2}\left(\beta_{1}=0,07\right)$ para 
distintos valores de $k_{31}$, se puede observar que el error aumenta linealmente con $\beta_{2}$ y que el error relativo aumenta al aumentar $k_{31}$. Los casos que se presentan corresponden a soluciones de las denominadas Tipo I.

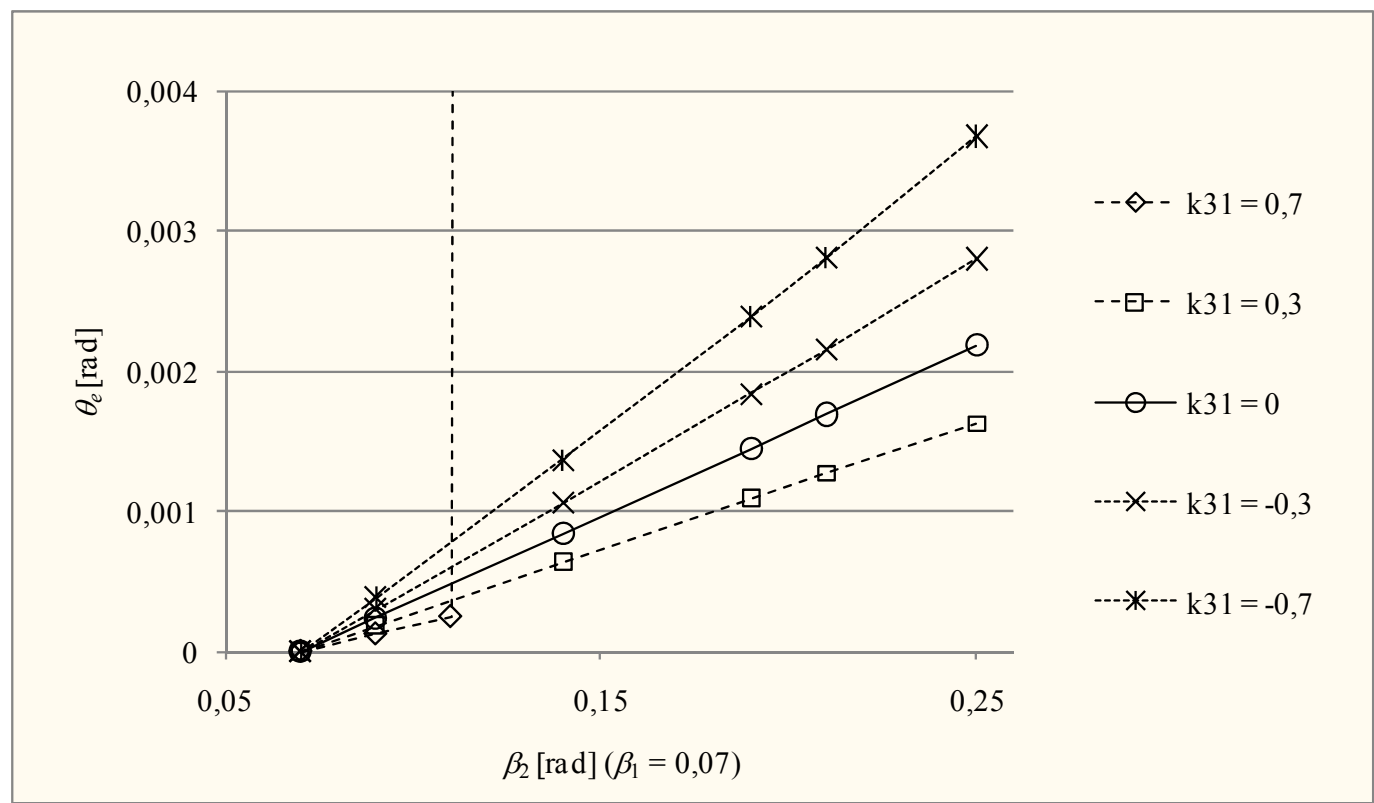

Figura 0.39 Ángulo de nutación de equilibrio, $\theta_{e}$, en función de $\beta_{2}\left(\beta_{1}=0,07\right)$ para distintos valores de $k_{31}$ que resultan de las simulaciones numéricas. 


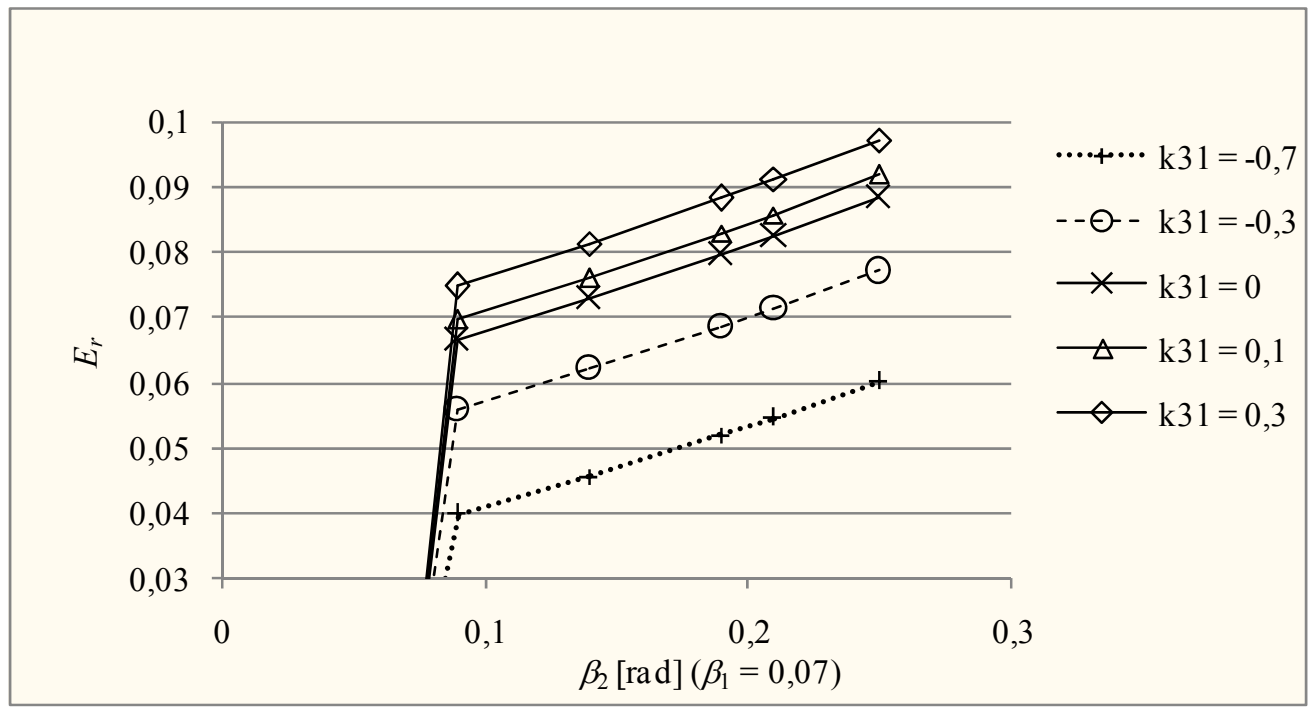

Figura 0.40 Error relativo, $E_{r}$, entre el ángulo de nutación de equilibrio, $\theta_{e}$, de la solución del modelo analítico y de la simulación numérica, en función de $\beta_{2}\left(\beta_{1}=0,07\right)$, para distintos valores de $k_{31}$.

\subsubsection{Solución Tipo II}

Para describir las soluciones Tipo II (ciclo límite estable) se considera el caso $k_{31}=0,7$, y $\beta_{2}=$ 0,21 (con $\beta_{1}=0,07$ ). Se observa que la solución para este caso muestra un comportamiento senoidal de las variables $\omega_{1}, \omega_{2}, \omega_{3}, U, \mathrm{y} \theta$, donde el ángulo de nutación de equilibrio $\theta$ se encuentra alejado del ángulo nulo (aproximadamente 0,41 radianes). En las Figuras 3.41 a 3.43 se puede observar el comportamiento senoidal de las variables $\omega_{1}, \omega_{2}, \omega_{3}$, apreciándose un cierto desfase entre $\omega_{1}$ y $\omega_{2}$. La velocidad $\omega_{3}$ es casi constante, $\omega_{3} \approx 294,8 \mathrm{rad} / \mathrm{s}$, con una fluctuación relativa menor a $3 \cdot 10^{-4}$.

En la Figura 3.44 se puede ver el comportamiento senoidal de la variable $U$. 


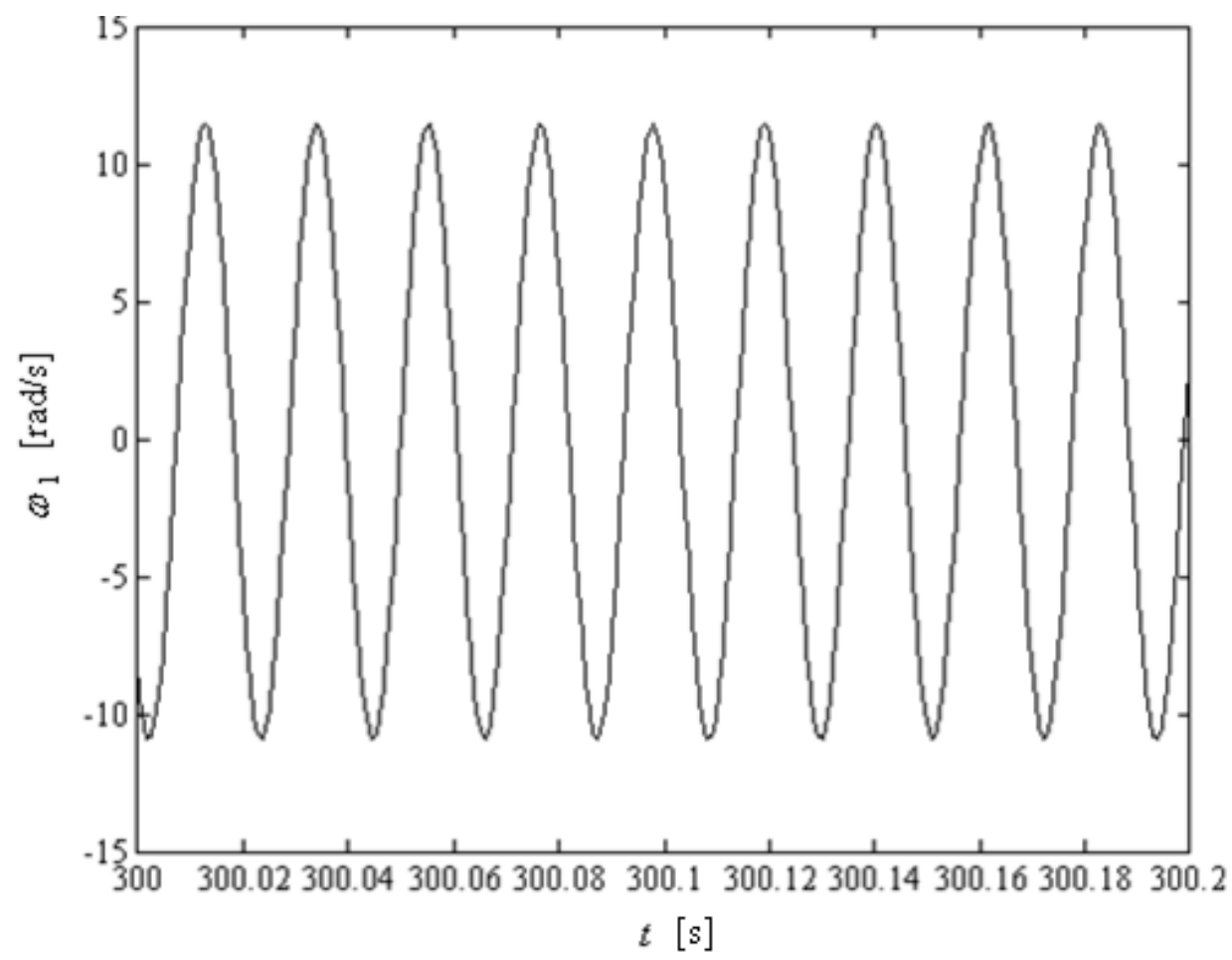

Figura 0.41 Variación de la velocidad angular, $\omega_{1}$, en función del tiempo, $t$, para el caso que $\beta_{2}=0,21\left(\operatorname{con} \beta_{1}=0,07\right.$ y $\left.k_{31}=0,7\right)$. 


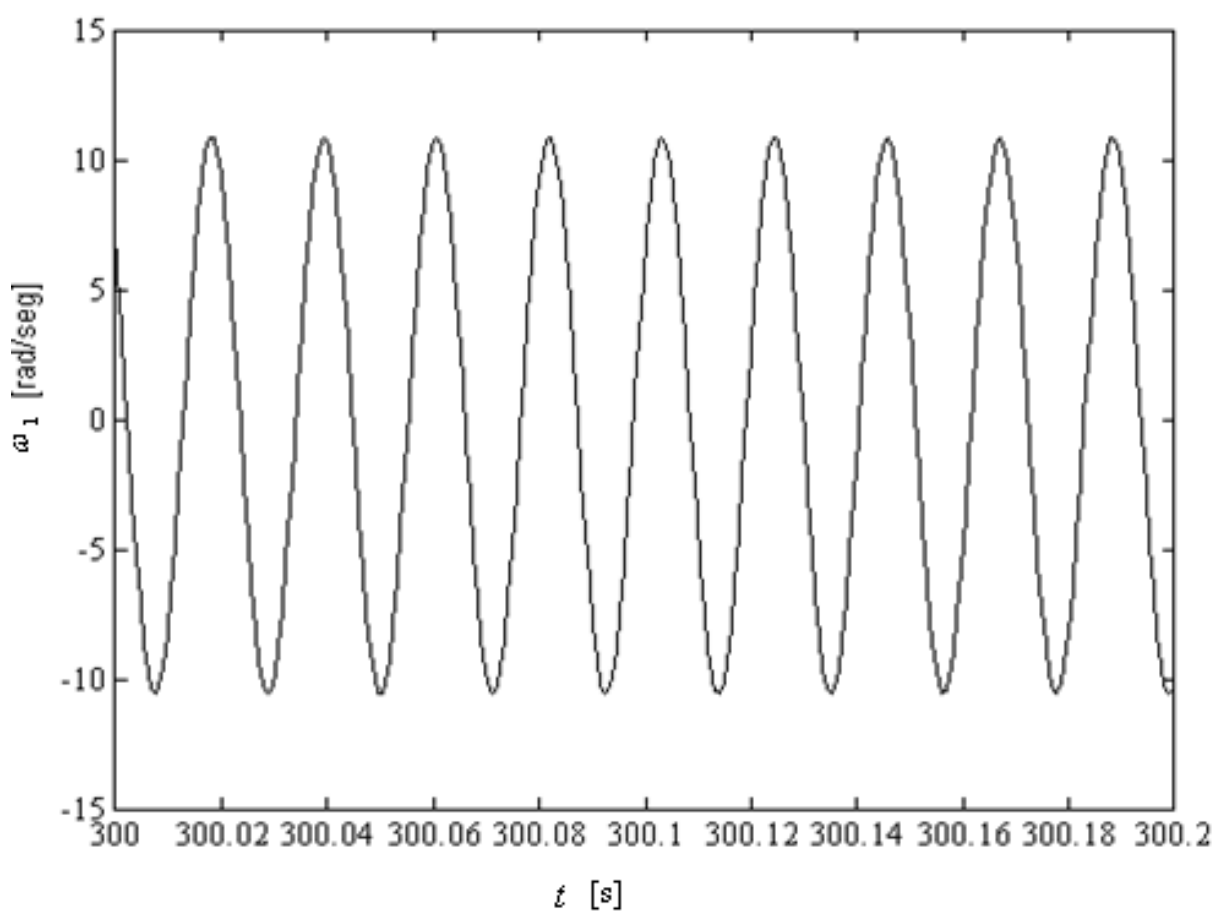

Figura 0.42 Variación de la velocidad angular, $\omega_{2}$, en función del tiempo, $t$, para el caso que $\beta_{2}=0,21\left(\operatorname{con} \beta_{1}=0,07\right.$ y $\left.k_{31}=-0,7\right)$. 


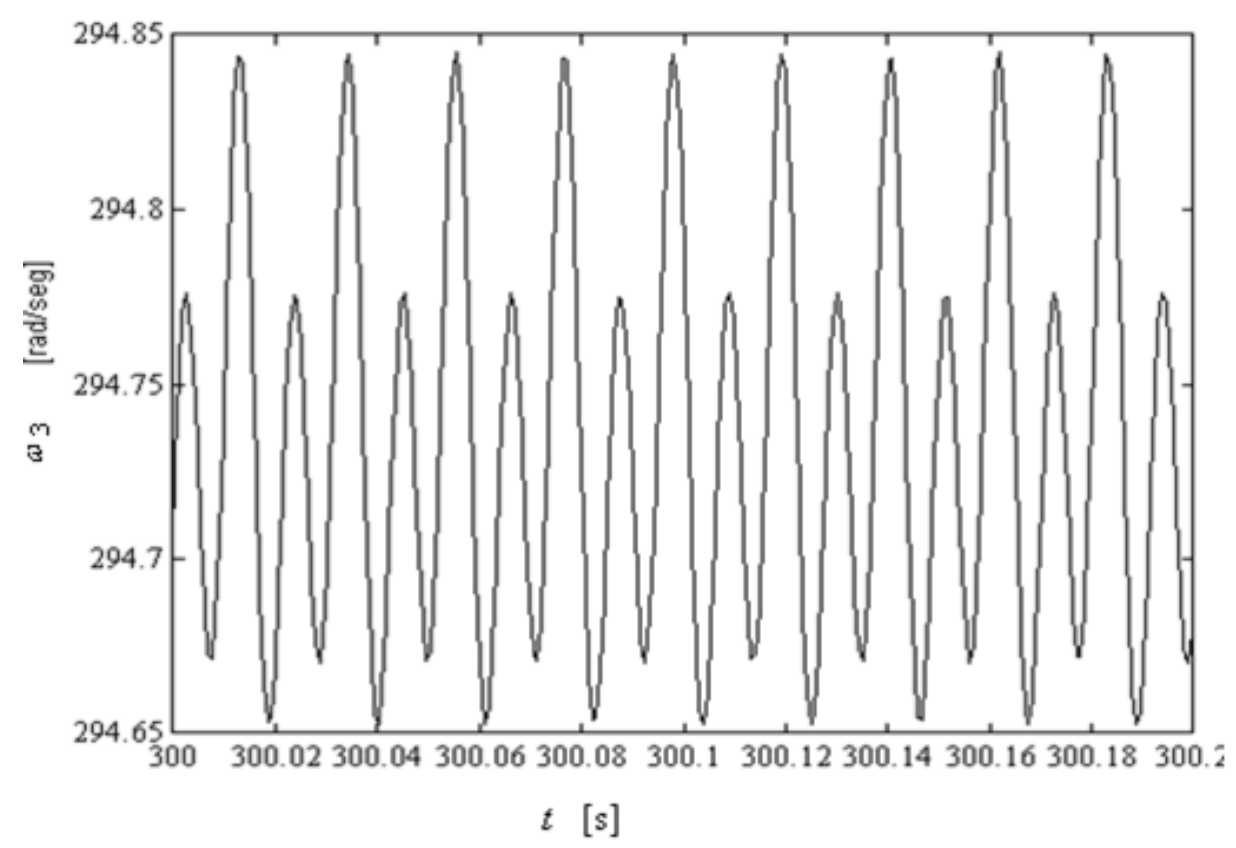

Figura 0.43 Variación de la velocidad angular, $\omega_{3}$, en función del tiempo, $t$, para el caso que $\beta_{2}=0,21\left(\operatorname{con} \beta_{1}=0,07\right.$ y $\left.k_{31}=-0,7\right)$. 


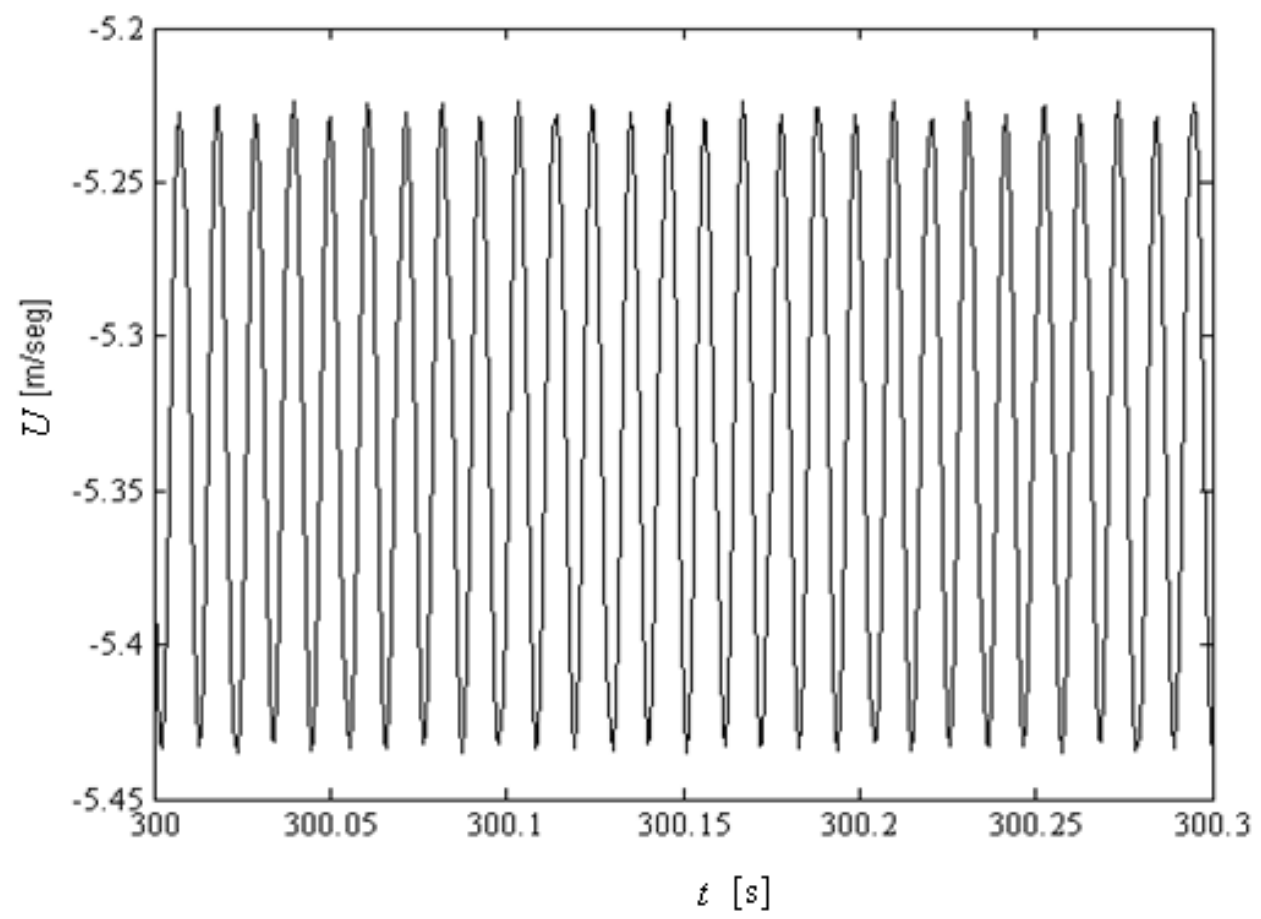

Figura 0.44 Variación de la velocidad vertical en coordenadas inerciales, $U$, para el caso que $\beta_{2}=0,21\left(\operatorname{con} \beta_{1}=0,07\right.$ y $\left.k_{31}=-0,7\right)$ con el tiempo, $t$.

Si consideramos los ángulos de Euler, se puede observar en la Figura 3.45 el comportamiento de la nutación $\theta$ en función del tiempo, para una situación de equilibrio dinámico. El comportamiento que aparece es senoidal, donde el valor medio es de 0,437 radianes con una amplitud de 0,019 radianes. Por tanto el ángulo de nutación es casi constante, con una fluctuación de pequeña amplitud (un 4\% del valor medio).

Se observa en la Figura 3.46, por otro lado, que el ángulo de rotación propia, $\varphi$, aumenta a velocidad aproximadamente constante (de magnitud 273,2 rad/s).

Por otro lado la evolución del ángulo de precesión en el tiempo se muestra en la Figura 3.47, donde se observa que aumenta a velocidad aproximadamente constante (de magnitud -0.584 $\mathrm{rad} / \mathrm{s})$. 


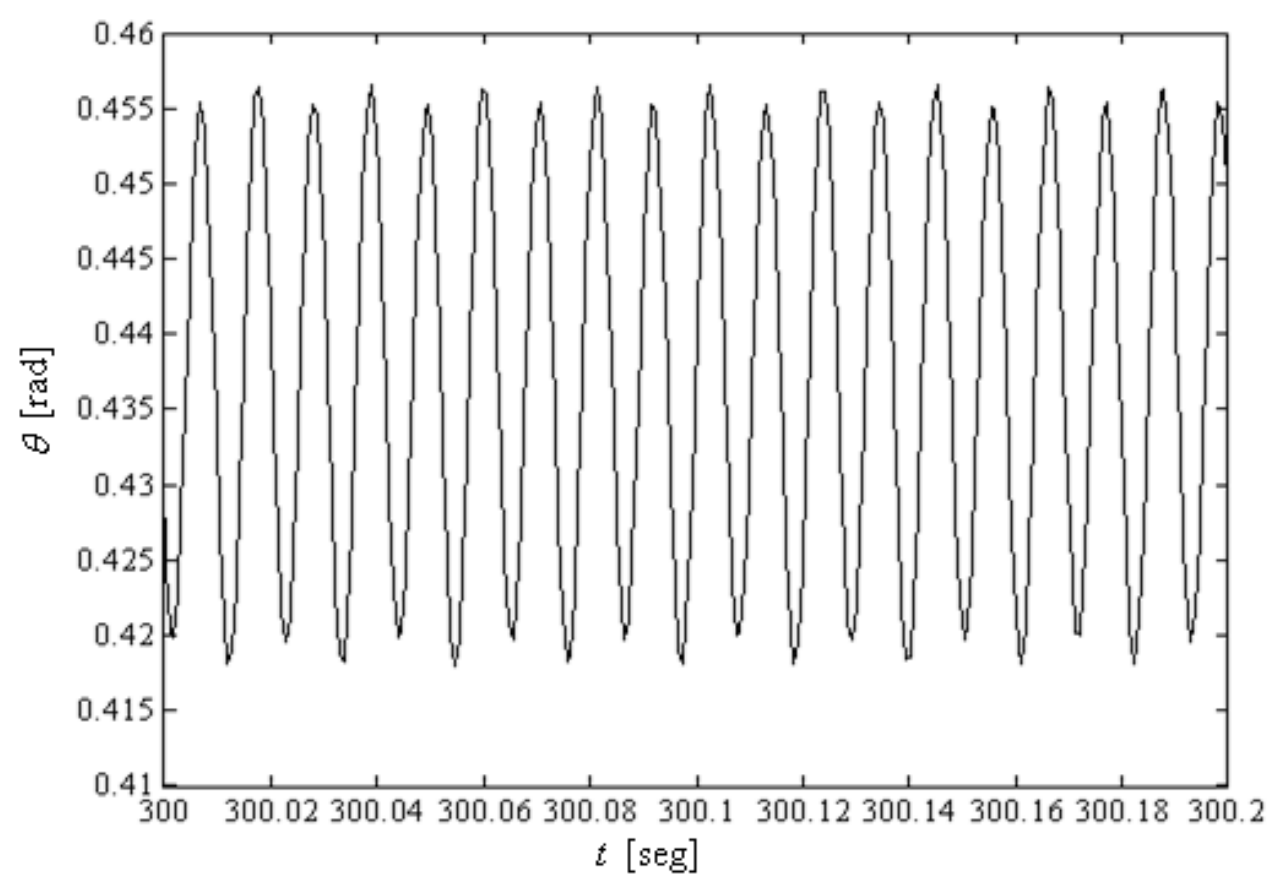

Figura 0.45 Variación del ángulo de nutación, $\theta$, en función del tiempo, $t$, para el caso que $\beta_{2}$ $=0,21\left(\operatorname{con} \beta_{1}=0,07\right.$ y $\left.k_{31}=0,7\right)$. 


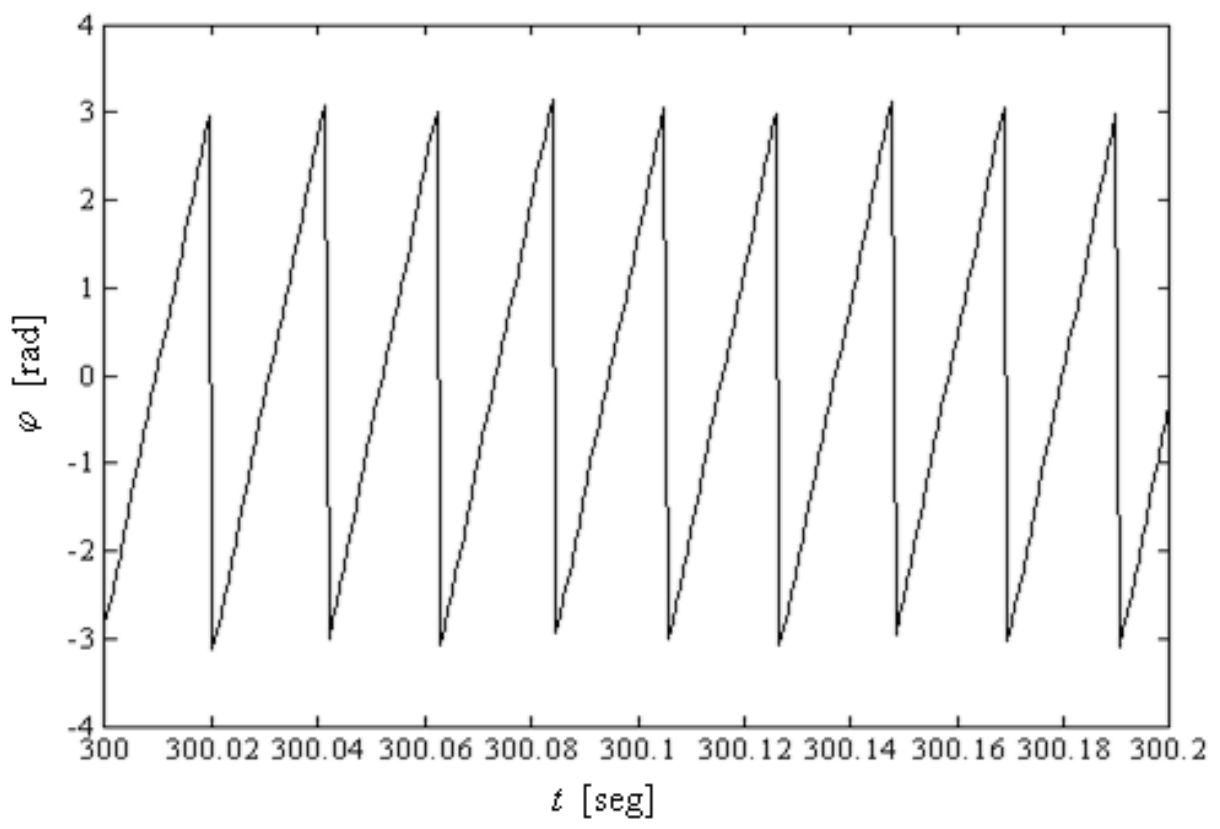

Figura 0.46 Variación del ángulo de rotación propia, $\varphi$, en función del tiempo, $t$, para el caso que $\beta_{2}=0,21\left(\operatorname{con} \beta_{1}=0,07\right.$ y $\left.k_{31}=0,7\right)$, para un dominio entre $-\pi$ y $\pi$. 


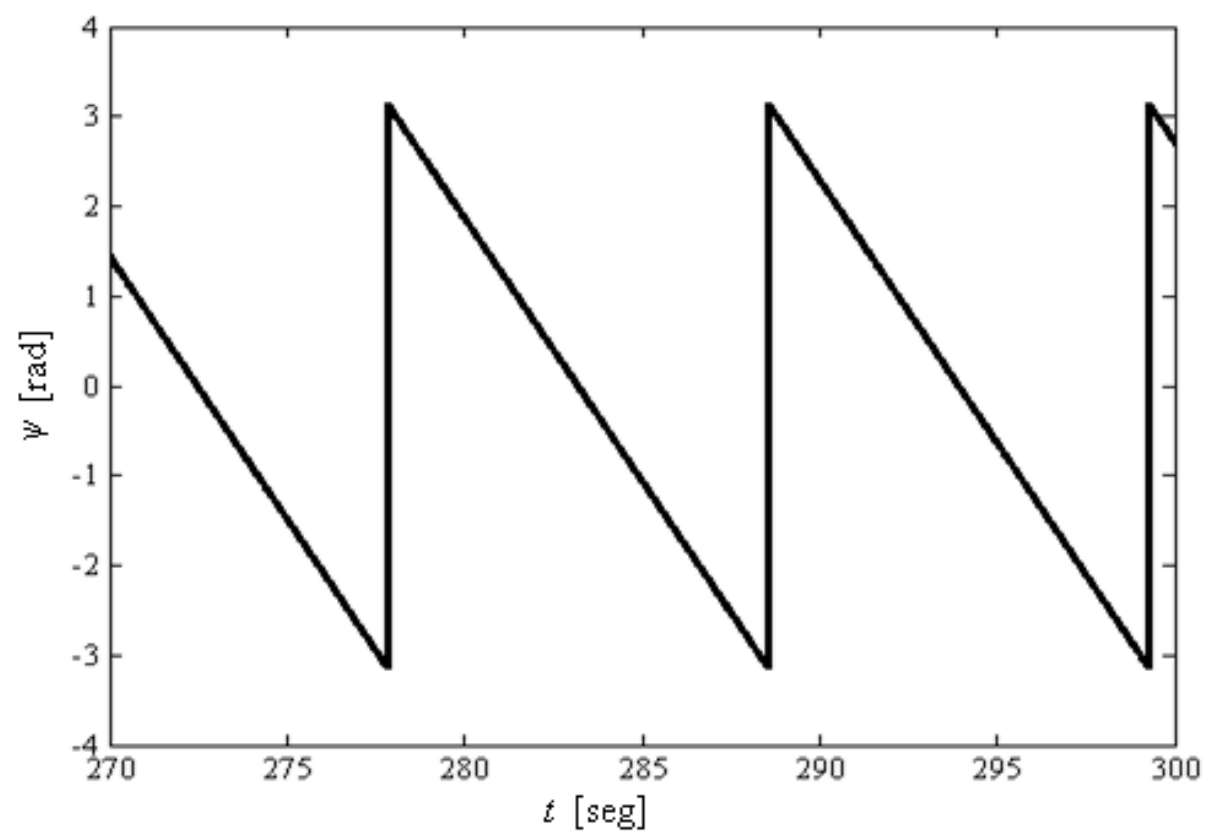

Figura 0.47 Variación del ángulo de precesión, $\psi$, en función del tiempo, $t$, para el caso que $\beta_{2}=0,21\left(\operatorname{con} \beta_{1}=0,07\right.$ y $\left.k_{31}=0,7\right)$, para un dominio entre $-\pi \mathrm{y} \pi$.

Si consideramos la velocidad angular en el plano 1, 2 en coordenadas del cuerpo, $\omega_{12}$, vemos que esta se mantiene aproximadamente constante con una fluctuación de 4\%, como se aprecia en la Figura 3.48.

Si se grafica la función atan $\left(\omega_{1} / \omega_{2}\right)$, que representa el ángulo del vector $\omega_{12}$ con el eje 2 , podemos ver que el ángulo de la velocidad de rotación transversal crece linealmente con el tiempo, es decir $\omega_{12}$ gira a velocidad constante. Para las hipótesis realizadas en el capítulo correspondiente al desarrollo del modelo analítico este ángulo es igual a $\varphi$. Este ángulo se presenta en la Figura 3.49.

En el caso que se grafique el ángulo dado por atan $\left(\omega_{12} / \omega_{3}\right)$, como se presenta en la Figura 3.50 , se puede ver que este ángulo es aproximadamente constante con una fluctuación cercana 
al 8\%. Este ángulo representa el ángulo del vector $\omega$ visto en ejes cuerpo en relación al eje 3. En la solución Tipo I este ángulo es $\theta_{e}$.

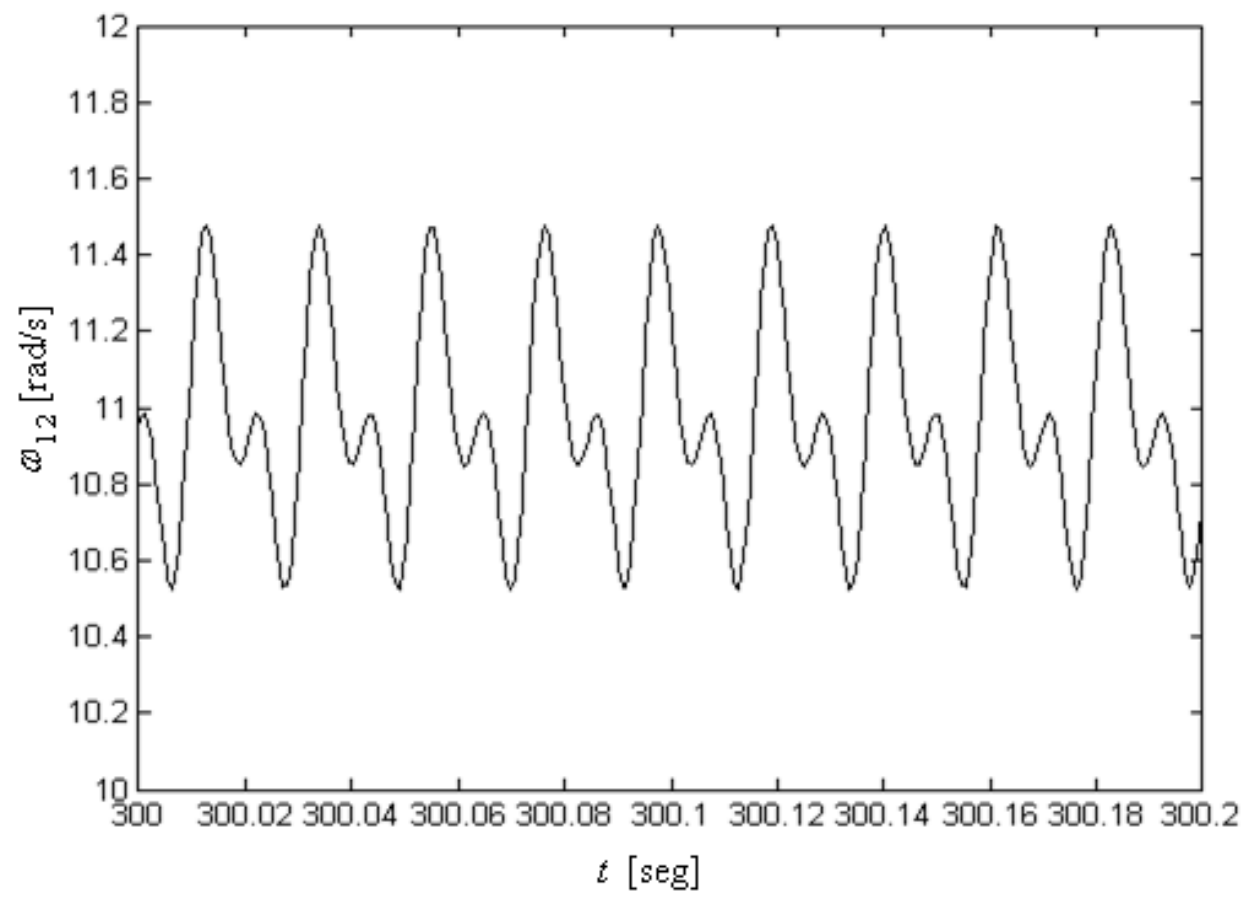

Figura 0.48 Variación de la velocidad angular, $\omega_{12}$, en función del tiempo, $t$, para el caso que $\beta_{2}=0,21\left(\operatorname{con} \beta_{1}=0,07\right.$ y $\left.k_{31}=0,7\right)$. 


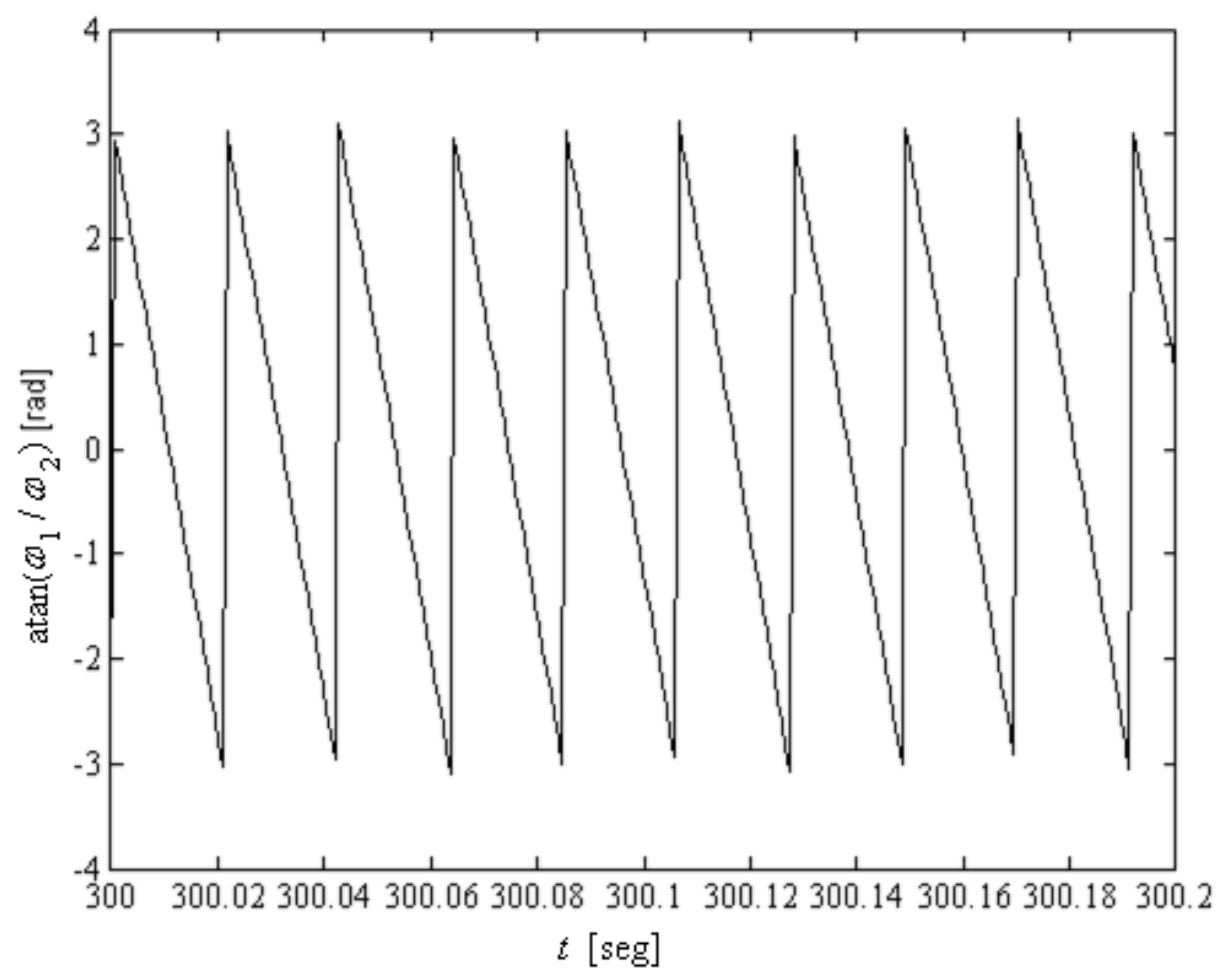

Figura 0.49 Variación del ángulo dado por atan $\left(\omega_{1} / \omega_{2}\right)$ en función del tiempo, $t$, para el caso que $\beta_{2}=0,21\left(\operatorname{con} \beta_{1}=0,07\right.$ y $\left.k_{31}=0,7\right)$, para un dominio entre $-\pi \mathrm{y} \pi$. 


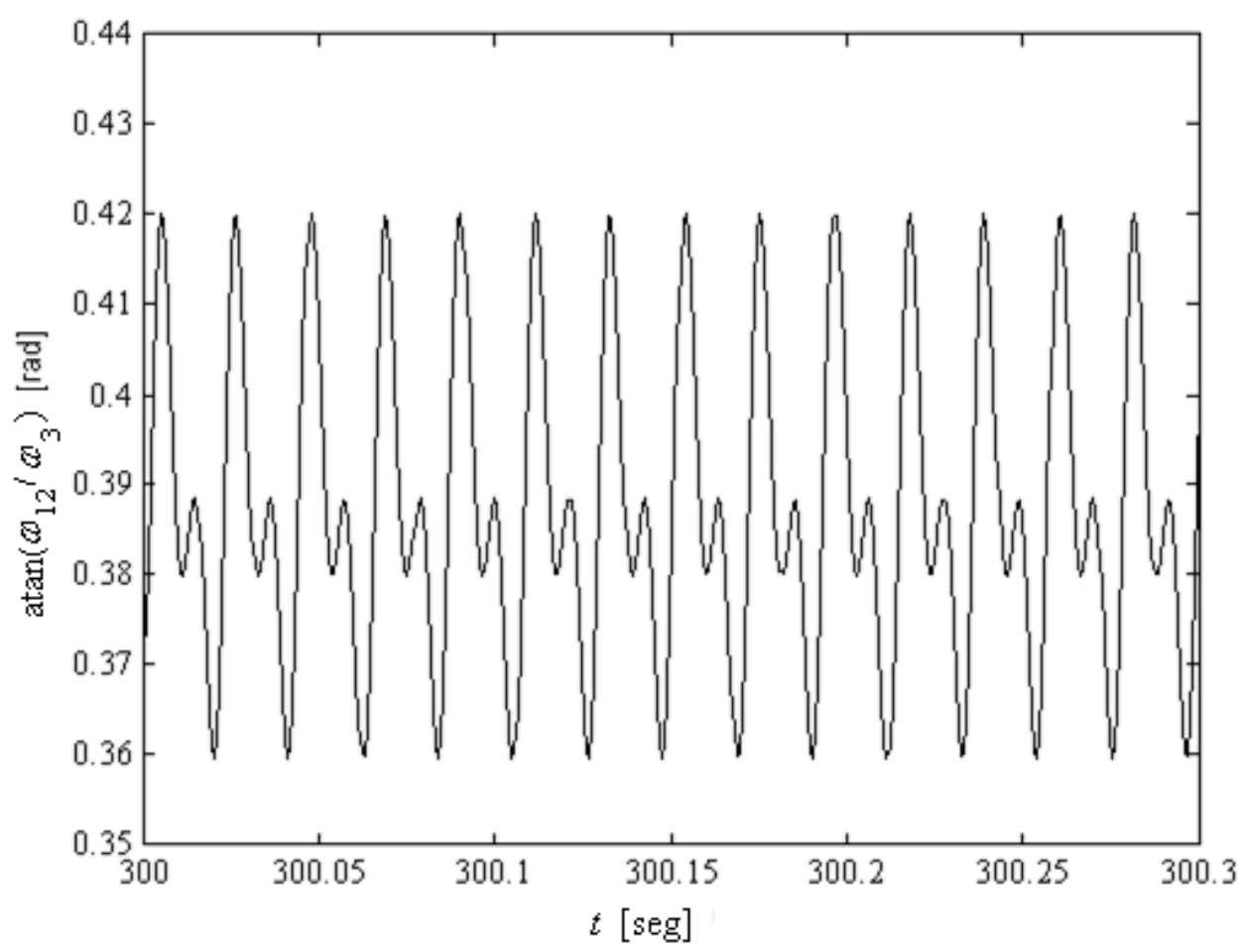

Figura 0.50 Variación del ángulo dado por atan $\left(\omega_{12} / \omega_{3}\right)$ en función del tiempo, $t$, para el caso que $\beta_{2}=0,21\left(\operatorname{con} \beta_{1}=0,07\right.$ y $\left.k_{31}=0,7\right)$.

En la Figura 3.51 se representa la componente $\omega_{2}$ en función de $\omega_{1}$ para un lapso de tiempo acotado. La figura que forma el gráfico es asimilable a una elipse de semiejes 10,5 rad/s y $11,5 \mathrm{rad} / \mathrm{s}$. Como la excentricidad es pequeña, el movimiento se parece al de un cuerpo girando en el vacío (sin momentos aplicados), cuya solución es $\left|\omega_{12}\right|=$ cte. 


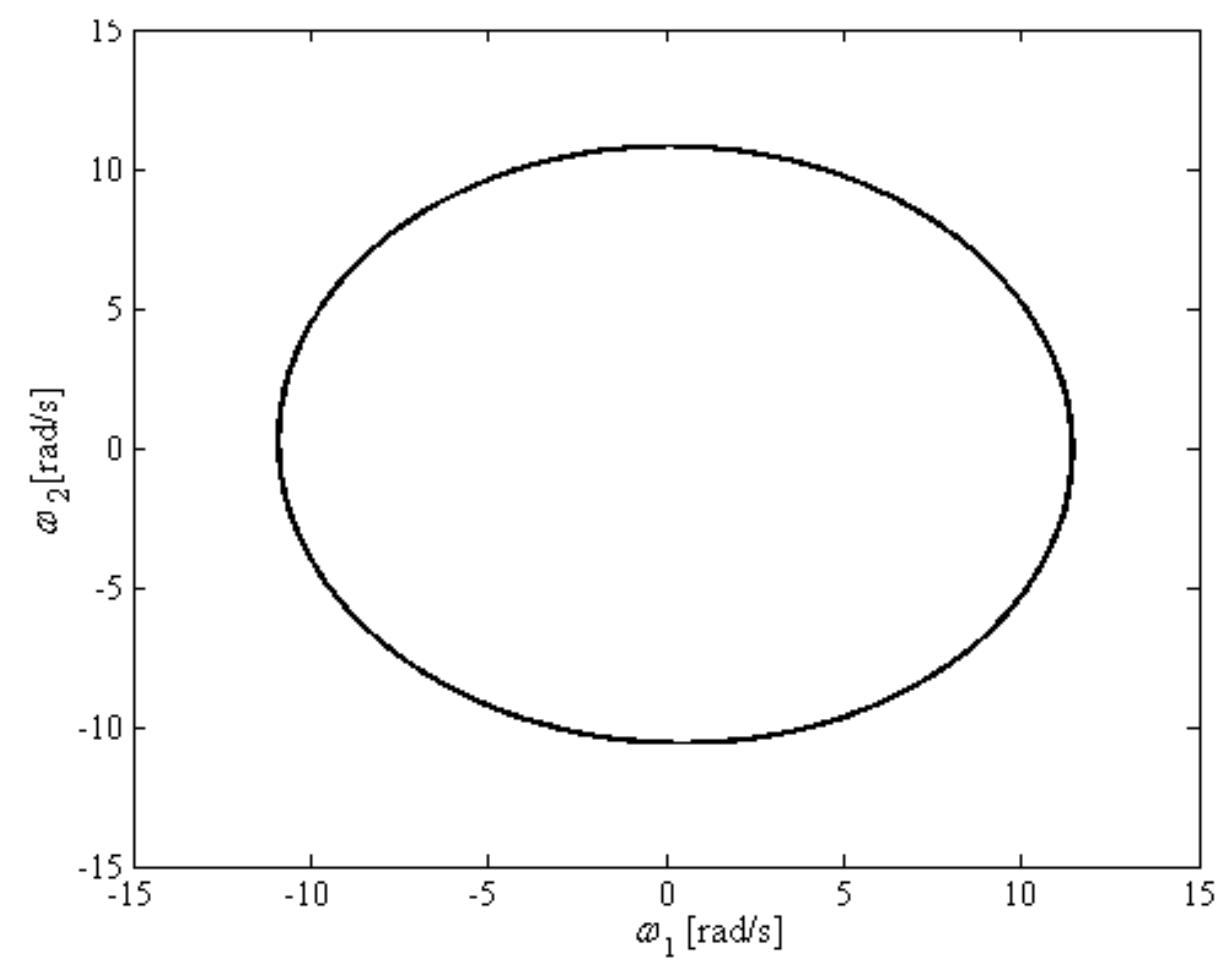

Figura 0.51 Variación de la velocidad angular $\omega_{2}$ y $\omega_{1}$ en un lapso de tiempo para el caso que $\beta_{2}=0,21\left(\operatorname{con} \beta_{1}=0,07\right.$ y $\left.k_{31}=0,7\right)$.

La solución que se denomina Tipo I, o la solución de referencia, predicha por el modelo analítico, se puede describir como una solución estática donde se orienta el pararrotor $(\theta, \varphi)$ de forma que $\dot{\theta}=\dot{\varphi}=0$. En esa orientación aparece el giro de precesión $\dot{\psi}=$ cte. El par de fuerzas que aparece (debido a la desalineación entre la sustentación y el peso) es el que hace que el momento cinético vaya girando a la misma velocidad que el cuerpo, $\dot{\psi}$, de forma que $\omega$ permanece fijo según el eje inercial $\mathrm{Z}$.

La solución que se denomina Tipo II puede describirse como un caso en el que el cuerpo tiene un giro propio $\dot{\varphi}$ muy rápido y una precesión $\dot{\psi}$ lenta, $\dot{\psi} \ll \dot{\varphi}$. El momento cinético estará 
dirigido según aproximadamente el eje 3. El hecho de que este tipo de solución aparece cuando $k_{31} \neq 0$ sugiere que al estar la resultante de la sustentación (promediada en una vuelta) en el centro del pararrotor, pero por encima del centro de masas, cuando hay rotación, da lugar (junto al peso) a un par de fuerzas perpendicular al momento cinético, ocasionando un movimiento de precesión. El hecho de que $\dot{\boldsymbol{\omega}}_{12}$ describa una cuasi elipse, con una excentricidad muy pequeña, es decir, parecida a una circunferencia (que es el movimiento de $\dot{\boldsymbol{\omega}}_{12}$ en el caso de un cuerpo libre de momentos exteriores) indica que el momento aerodinámico a lo largo de una vuelta es casi constante.

La velocidad de precesión $\dot{\psi}$ se puede estimar a partir de la expresión que da el efecto del par exterior en el cambio del momento cinético:

$m g d \sin \theta=\left(I_{3} \dot{\varphi}\right) \dot{\psi}$

donde $d$ es la distancia entre el centro de masas y el punto del eje donde están las palas. Para el caso que $k_{31}=0,7, \mathrm{y} \beta_{2}=0,21$ (con $\beta_{1}=0,07$ ) la expresión (170) arroja un valor de $\dot{\psi}=$ 0,53, que es un 5\% menor al arrojado de la simulación numérica, ratificándose así su calidad como estimador.

\subsubsection{Influencia de los parámetros de la configuración}

Para analizar el efecto de distintos parámetros geométricos y aerodinámicos en la estabilidad del sistema se considera un ángulo de nutación inicial de 0,1 radianes, y se observa la evolución del ángulo de nutación. Se computa el tiempo desde el instante en que se alcanza el equilibrio en una banda de $\pm 0,01$ radianes. En la Tabla 3.19 se muestran los valores de tiempo, los ángulos de nutación medios de equilibrio para distintos casos de paso de palas y posición del centro de masas $k_{31}$. Se presenta para el primer grupo de casos propuestos $\left(\delta_{\beta}=\right.$ $0,01)$ de la derivada en el tiempo del ángulo $\varphi$ de equilibrio. 
Tabla 0.19 Tiempo de establecimiento de las soluciones de equilibrio, $\boldsymbol{t}_{e e}$, para distintas variantes de pararrotor sobre el caso base, según el tipo de solución.

\begin{tabular}{|c|c|c|c|c|c|c|c|}
\hline \multirow{2}{*}{\begin{tabular}{|c|} 
Casos \\
Parámetros \\
\end{tabular}} & \multicolumn{3}{|c|}{$\begin{array}{l}\beta_{1}=\mathbf{0 , 0 1} \\
\beta_{2}=\mathbf{0 , 0 1}\end{array}$} & \multicolumn{2}{|c|}{$\begin{array}{l}\beta_{1}=\mathbf{0 , 0 7} \\
\beta_{2}=\mathbf{0 , 0 7}\end{array}$} & \multicolumn{2}{|c|}{$\begin{array}{l}\beta_{1}=\mathbf{0 , 0 7} \\
\beta_{2}=\mathbf{0 , 1 4}\end{array}$} \\
\hline & $\begin{array}{l}t_{e e} \\
{[\mathbf{s}]}\end{array}$ & $\begin{array}{c}\mathrm{d} \varphi_{e} / \mathrm{dt} \text { medi } \\
{[\mathrm{rad} / \mathrm{s}]}\end{array}$ & $\begin{array}{c}\theta e_{e} \text { medio } \\
{\left[{ }^{\circ}\right]}\end{array}$ & $\begin{array}{l}t_{e e} \\
{[\mathbf{s}]}\end{array}$ & $\begin{array}{c}\theta e_{e} \text { medio } \\
{\left[^{\circ}\right]}\end{array}$ & $\begin{array}{l}t_{e e} \\
{[\mathbf{s}]}\end{array}$ & $\begin{array}{c}\theta e_{e} \text { medio } \\
{\left[^{\circ}\right]}\end{array}$ \\
\hline$k_{31}=1,0$ & 63,2 & $-0,41$ & $34,3^{\mathrm{II}}$ & 49,9 & $38,7^{\mathrm{II}}$ & 44,4 & $41,3^{\mathrm{II}}$ \\
\hline$k_{31}=0,5$ & 30,1 & $-0,66^{*}$ & $0,0^{\mathrm{I}}$ & 32,2 & $0,0^{\mathrm{I}}$ & 37,5 & $0,0^{\mathrm{I}}$ \\
\hline$k_{31}=0$ & 7,8 & $-0,61 *$ & $0,0^{\mathrm{I}}$ & 8,1 & $0,0^{\mathrm{I}}$ & 8,5 & $0,0^{\mathrm{I}}$ \\
\hline$k_{31}=-0,5$ & 6,8 & $0,30 *$ & $0,0^{\mathrm{I}}$ & 7,9 & $0,0^{\mathrm{I}}$ & 9,6 & $0,0^{\mathrm{I}}$ \\
\hline$k_{31}=-1,0$ & 14,3 & $0,35^{*}$ & $0,0^{\mathrm{I}}$ & 24,1 & $0,0^{\mathrm{I}}$ & 47,5 & $0,0^{\mathrm{I}}$ \\
\hline$k_{31}=-2,0$ & 43,5 & 0,52 & $39,2^{\mathrm{II}}$ & 28,2 & $45,4^{\mathrm{II}}$ & 23,5 & $48,1^{\mathrm{II}}$ \\
\hline
\end{tabular}

${ }^{\mathrm{I}}$ Solución Tipo I. Para los casos donde $\delta_{\beta} \neq 0$, los ángulos de equilibrio son mayores a 0 y menores a 0,05 grados.

${ }^{\text {II }}$ Solución Tipo II

*La velocidad del ángulo de Euler $\varphi$ no tiene sentido físico cuando $\theta_{e}=0$. En las simulaciones numéricas este parámetro se computa en función de los valores infinitesimales que toma $\theta$.

La simulación numérica muestra en la Tabla 3.19 que un aumento en el paso de las palas y un aumento en $\delta_{\beta}$ hacen aumentar el tiempo de evolución del sistema hasta alcanzar el equilibrio en todos los casos.

Por otro lado la simulación numérica muestra, cualitativamente, la existencia de dos tipos de solución, Tipo I y Tipo II, que fueron presentados anteriormente. Este tipo de soluciones se han denominado en trabajos anteriores como cónicas (Levin y Shpund, 1995; Karlen, 1991). Finalmente otra alternativa de solución que se presenta es cuando el pararrotor se inclina más de 1,57 radianes (90 grados). A esta condición la caracterizaremos como solución inestable, de inestabilidad funcional, por cuanto el ángulo de nutación de equilibrio evoluciona a estados de equilibrio mayores o iguales a 90 grados, lo cual no es deseable desde el punto de vista funcional. En este caso la posición del centro de masas sobre eje 3 cambia mediante la 
rotación mencionada (se invierte) y evoluciona asimilándose a una solución Tipo II con $k_{31}$ de signo contrario (el centro de masas pasa de estar por encima del plano que contiene a las placas a estar por debajo).

Se puede observar que las soluciones Tipo I, con ángulo de nutación de equilibrio cero o muy cercano a cero, se alcanzan cuando $k_{31}$ es pequeño y/o $\delta_{\beta}$ es 0 o cercano a 0 . En estos casos la variación del tiempo de evolución a la solución de equilibrio resulta dependiente del paso de las palas y del parámetro $k_{31}$. Es posible observar el aumento del tiempo con el aumento de $k_{31}$.

Un aumento del valor absoluto de $k_{31}$ propicia la aparición de soluciones Tipo II. Una vez alcanzada una solución Tipo II, un aumento del valor de $k_{31}$ produce una disminución del tiempo en el que se alcanza la solución de equilibrio. Se puede observar en la Figura 3.52 la evolución del ángulo de nutación, $\theta$, en el tiempo para distintos valores del parámetro $k_{31}$. En este gráfico se pueden observar las distintas soluciones que se mencionan.

La Figura 3.53, 3.54 y 3.55 muestran la evolución de las velocidades angulares en coordenadas solidarias al cuerpo, $\omega_{1}, \omega_{2}, \omega_{3}$, en el tiempo para el caso $\beta_{1}=\beta_{2}=0,07$ y $k_{31}=0$, con ángulo de nutación inicial es $\theta=0,1$ radianes, y condiciones iniciales $\omega_{1}=0, \omega_{2}=0, \omega_{3}=$ $292,8 \mathrm{rad} / \mathrm{s}$. 


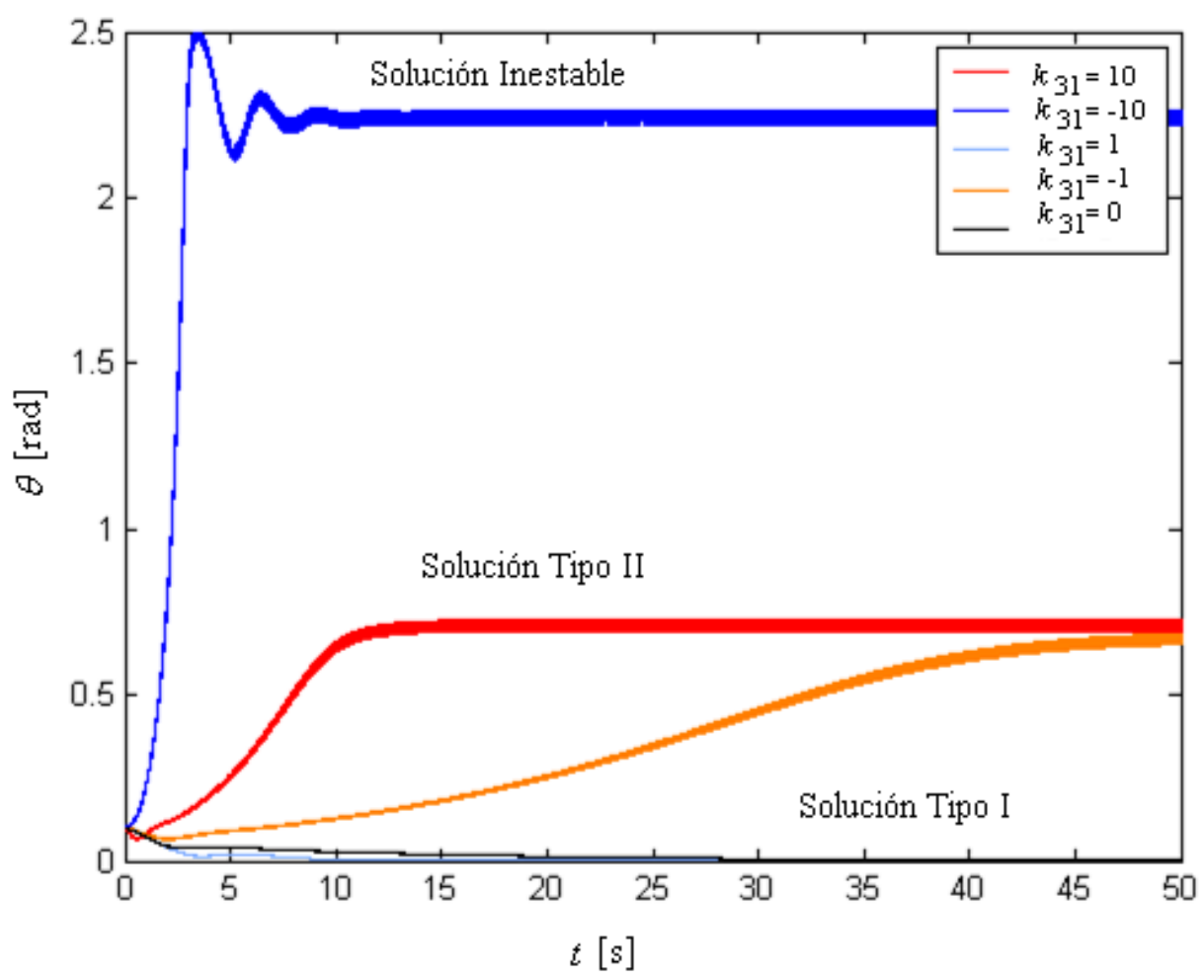

Figura 0.52 Variación en el tiempo, $t$, del ángulo de nutación para distintos valores de $k_{31}$. El ángulo de nutación inicial es $\theta=0,1$ radianes. Tipos de solución: I, II e inestable. 


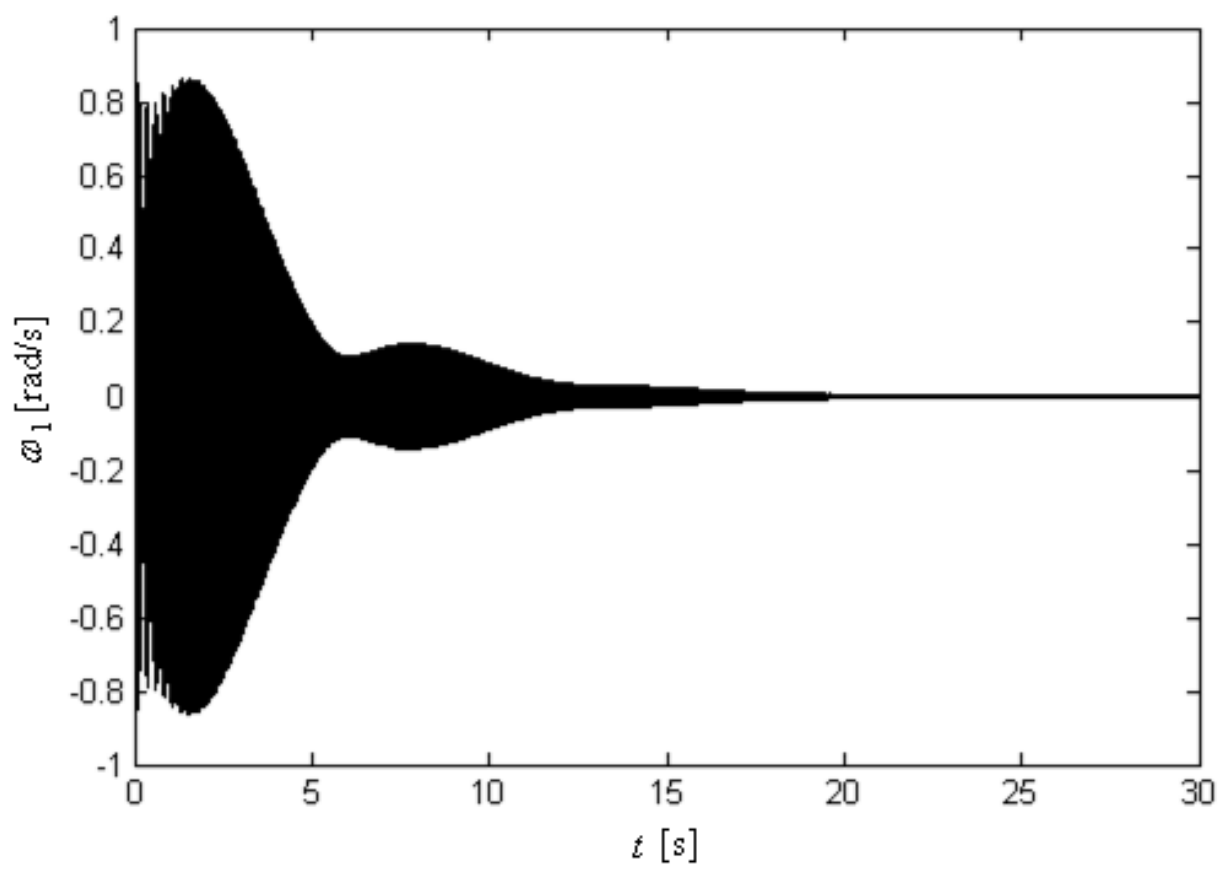

Figura 0.53 Variación con el tiempo, $t$, de la componente de la velocidad angular $\omega_{1}$, para el caso que $\beta_{1}=\beta_{2}=0,07$ y $k_{31}=0$. 


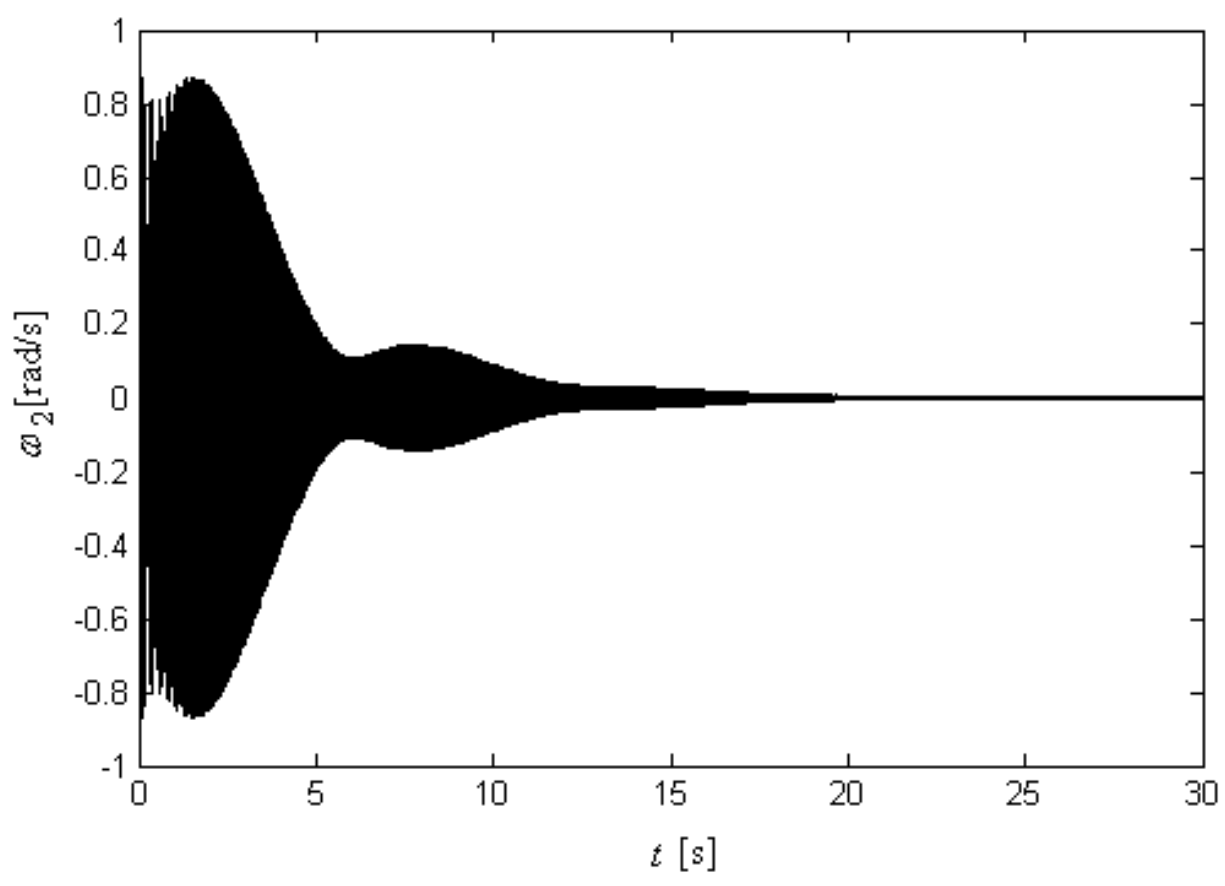

Figura 0.54 Variación con el tiempo, $t$, de la componente de la velocidad angular $\omega_{2}$, para el caso que $\beta_{1}=\beta_{2}=0,07$ y $k_{31}=0$. 


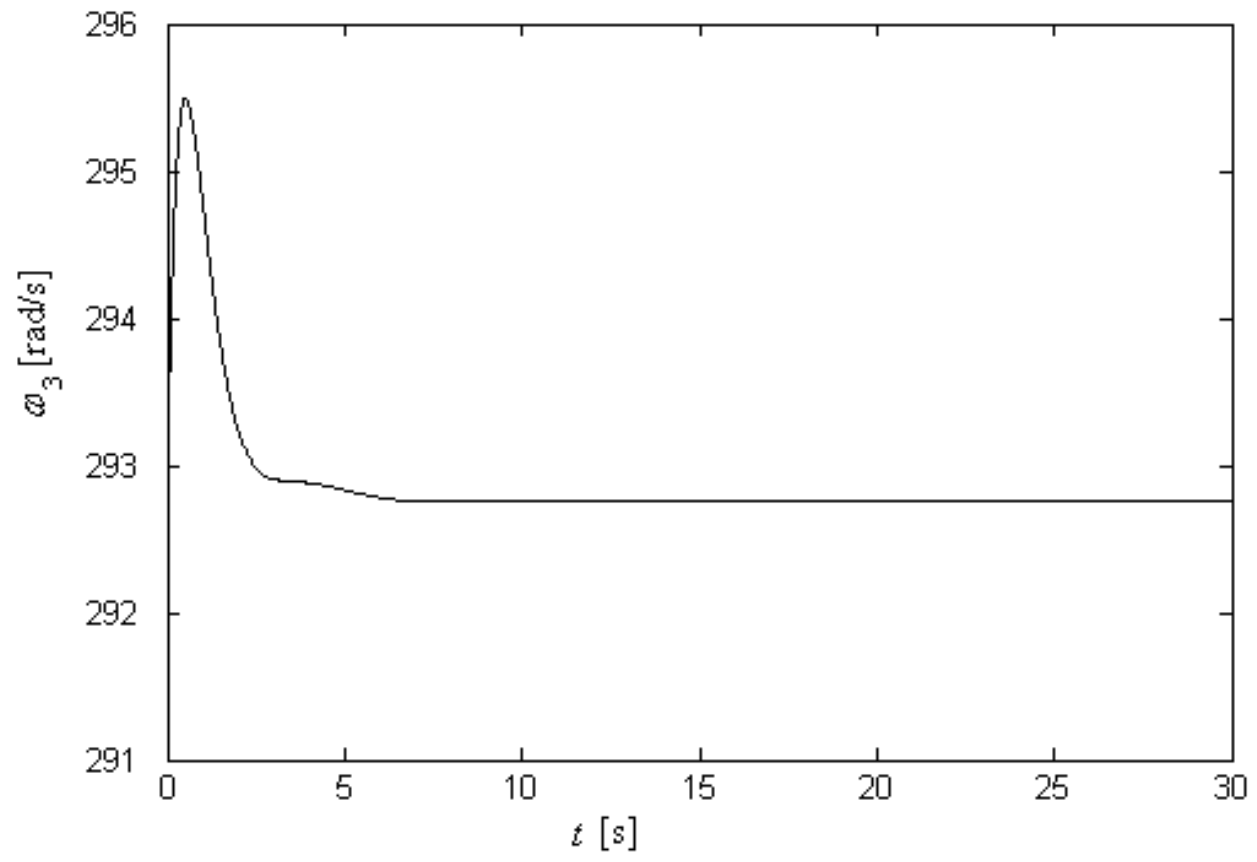

Figura 0.55 Variación con el tiempo, $t$, de la componente de la velocidad angular $\omega_{3}$, para el caso que $\beta_{1}=\beta_{2}=0,07$ y $k_{31}=0$.

Se define adicionalmente el coeficiente de resistencia del modelo, $c_{D M}$, tomando como área de referencia a la superficie de las dos palas, adoptando la expresión:

$c_{D M}=\frac{T}{\rho V_{v}^{2} S}$.

Se define $k$ a la relación de velocidades:

$k=\frac{V_{v}}{\omega r_{11}}$,

donde $R=r_{11}=r_{12}$.

Se simulan una serie de variantes sobre el Caso Base a los fines de determinar el efecto del cambio de $\beta_{0}$, que se muestran en la Tabla 3.20. Al respecto de los resultados obtenidos por 
Nadal Mora (2005) los valores para ángulos de paso, $\beta_{0}$, positivos, los resultados se muestran de magnitudes comparables.

Con el objeto de permitir una comparación más adecuada, se presenta una configuración adicional de pararrotor, que se especifica en la Tabla 3.20, en la que la diferencia con el Caso Base es el cambio de la relación entre momentos de inercia, coeficientes aerodinámicos y distancia del centro de masas a los centros aerodinámico de presiones.

Tabla 0.20 Configuración adicional del ejemplo numérico.

\begin{tabular}{|c|c|c|}
\hline \multicolumn{3}{|c|}{ Parámetros generales } \\
\hline$C_{L \alpha}$ & 1,35 & $1 / \mathrm{rad}$ \\
\hline$C_{D}$ & 0,5 & - \\
\hline$\rho$ & 1,21 & $\mathrm{~kg} / \mathrm{m}^{3}$ \\
\hline$S$ & 0,012 & $\mathrm{~m}^{2}$ \\
\hline$r_{11}, r_{21}$ & 0,100 & $\mathrm{~m}$ \\
\hline$r_{12}, r_{22}$ & 0,034 & $\mathrm{~m}$ \\
\hline$\beta_{1}, \beta_{2}$ & 0,07 & $\mathrm{rad}$ \\
\hline$I_{1}$ & $6,3 \cdot 10^{-6}$ & $\mathrm{~kg} \mathrm{~m}^{2}$ \\
\hline$I_{2}$ & $22,1 \cdot 10^{-6}$ & $\mathrm{~kg} \mathrm{~m}^{2}$ \\
\hline$I_{3}$ & $56,3 \cdot 10^{-6}$ & $\mathrm{~kg} \mathrm{~m}^{2}$ \\
\hline
\end{tabular}

Tabla 0.21 $c_{D M}$ de equilibrio para distintos valores de $\beta_{0}$, para el Caso Base y el Caso Adicional.

\begin{tabular}{|l|c|c|c|c|}
\hline \multirow{2}{*}{ Detalle del caso } & \multicolumn{2}{|c|}{ Caso Base } & \multicolumn{2}{c|}{ Caso Adicional } \\
\cline { 2 - 5 } & $c_{D M}$ & $k$ & $c_{D M}$ & $k$ \\
\hline
\end{tabular}




\begin{tabular}{|c|c|c|c|c|}
\hline Caso Base $-\beta_{0}=-0,21$ & 17,48 & 0,296 & 19,16 & 0,277 \\
\hline Caso Base $-\beta_{0}=-0,14$ & 13,56 & 0,315 & 14,65 & 0,305 \\
\hline Caso Base $-\beta_{0}=-0,07$ & 10,41 & 0,346 & 12,17 & 0,337 \\
\hline Caso Base $-\beta_{0}=0$ & 7,98 & 0,382 & 8,50 & 0,372 \\
\hline Caso Base $-\beta_{0}=0,07$ & 6,13 & 0,422 & 6,48 & 0,412 \\
\hline Caso Base $-\beta_{0}=0,14$ & 4,72 & 0,467 & 4,96 & 0,457 \\
\hline Caso Base $-\beta_{0}=0,21$ & 3,66 & 0,518 & 3,82 & 0,501 \\
\hline
\end{tabular}

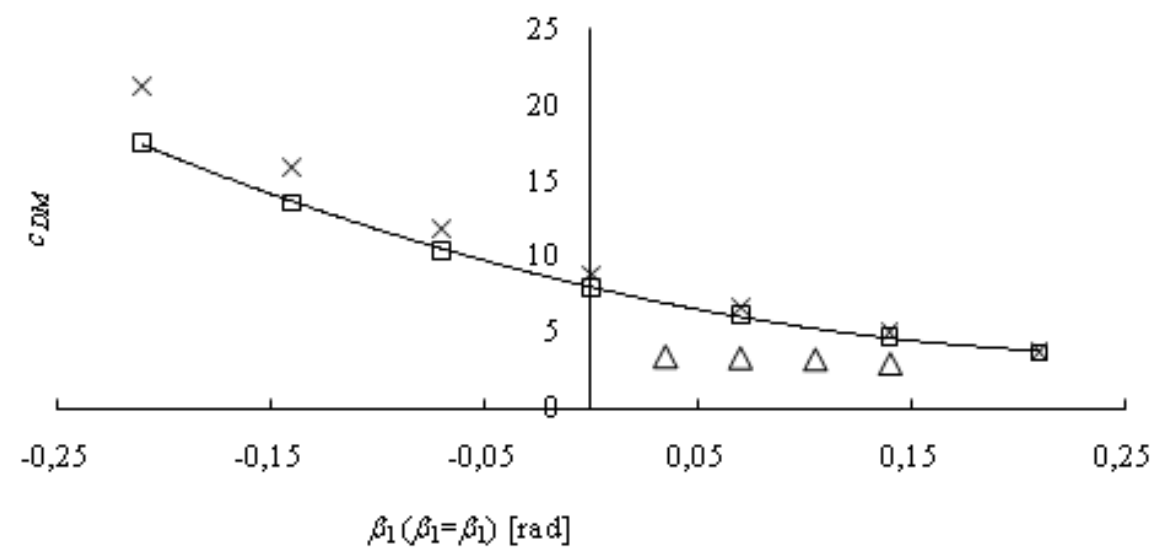

$\times$ Caso Adicional $\quad \square$ CasoBase $\quad \Delta$ CasoC01- NadalMora(2005)

Figura 0.56 Variación de $c_{D M}$ con $\beta_{1}\left(\beta_{1}=\beta_{2}\right)$ para $k_{31}=0$. Incluye una línea de tendencia polinomial. Se incluye en la figura además de Caso Base, el Caso Adicional presentado y datos del Nadal Mora (2005). 


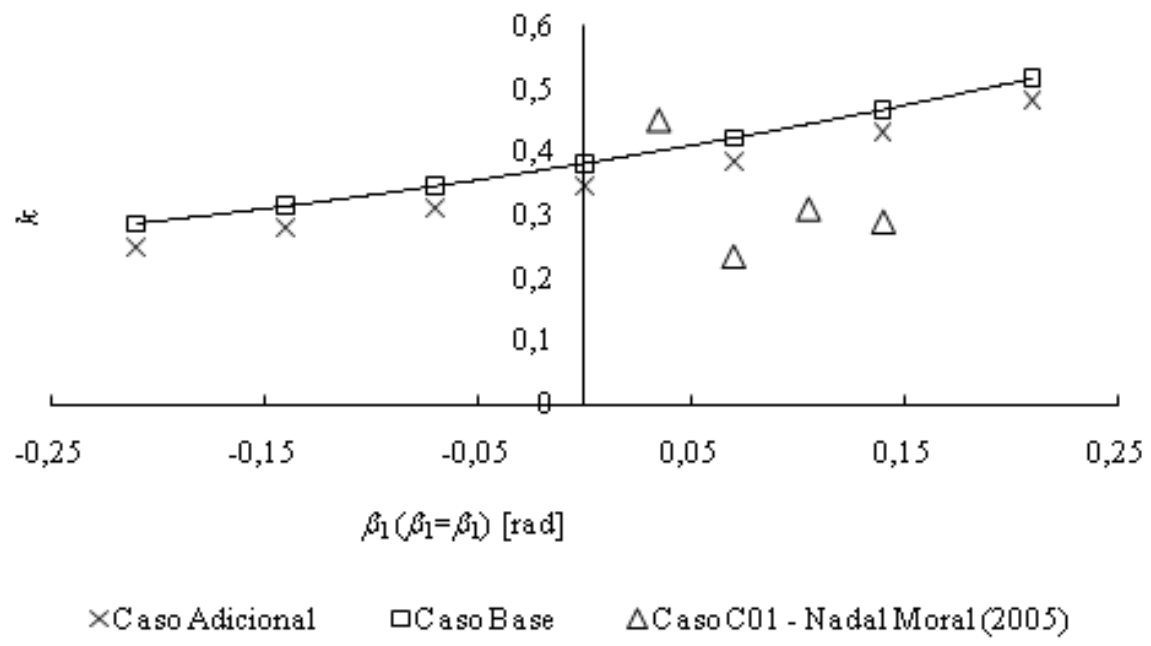

Figura 0.57 Gráfico de $k$ versus $\beta_{1}\left(\beta_{1}=\beta_{2}\right)$ con $k_{31}=0$. Incluye una línea de tendencia polinomial. Se incluye en la figura además de Caso Base, el Caso Adicional presentado y datos del Nadal Mora (2005).

Se observa en la Figura 3.57 de acuerdo a la tendencia que se evidencia en la curva que se incluye, que los valores de $c_{D M}$ aumentan al disminuir $\beta_{0}$. Adicionalmente se observa un mínimo absoluto para valores positivos de $\beta_{0}$.

Se observa en la Figura 3.57 de acuerdo a la tendencia que se evidencia en la curva que se incluye, que los valores de $k$ aumentan al aumentar $\beta_{0}$. La pendiente de la línea de tendencia incluida es $0,0961 /$ grado.

Sobre la base de la configuración presentada (a partir de ahora considerada como Caso Base) en la Tabla 3.20 se consideraron una serie de variantes con el fin de observar la influencia del cambio de los parámetros $k_{31}$ y $k_{21}$ asociados a la posición de las palas al respecto del centro de masas, la relación de momentos de inercia $I_{3} / I_{2}, I_{3} / I_{1}$ (sobre hipótesis que $I_{3}>I_{2} \geq I_{1}$ ) y la relación $C_{L \alpha} / C_{D}$ de las palas. A los fines de simplificar la presentación de las variantes se las dividió según el parámetro $k_{31}$. 


\subsubsection{Variantes con $k_{31}=0$}

En la Tabla 3.22 se presentan una selección de las variantes que fueron simuladas y su análisis. En todos los casos de corroboraron las observaciones realizadas con simulaciones accesorias.

Tabla 0.22 Comportamiento dinámico de variantes del caso con $k_{31}=0$ y parámetros $N_{e}{ }^{\prime}, k_{e}$.

\begin{tabular}{|c|c|c|c|c|c|c|}
\hline \multirow{2}{*}{$\begin{array}{l}\text { Número } \\
\text { de } \\
\text { Variante }\end{array}$} & \multirow[b]{2}{*}{ Detalle del caso } & \multirow[b]{2}{*}{$N_{e}{ }^{\prime}$} & \multirow[b]{2}{*}{$\boldsymbol{k}_{e}$} & \multirow{2}{*}{$\begin{array}{c}t_{e e^{*}} \\
{[\mathbf{s}]}\end{array}$} & \multicolumn{2}{|c|}{ Solución de Equilibrio } \\
\hline & & & & & $\begin{array}{l}\boldsymbol{\theta}_{e} * * \\
{[\mathbf{r a d}]}\end{array}$ & $\begin{array}{c}\boldsymbol{\theta}_{e} * * * \\
{[\mathbf{r a d}]}\end{array}$ \\
\hline 1 & Caso Base & 0,019 & 12,800 & 8,14 & 0,00 & 0,00 \\
\hline 2 & $\begin{array}{l}-k_{21}=0 \\
\text {-aumento de } I_{1} / I_{3} \\
(75 \%)\end{array}$ & 0,000 & 0,000 & 8,33 & 0,00 & 0,00 \\
\hline 3 & $\begin{array}{l}-k_{21}=0 \\
- \text { aumento de } I_{2} / I_{3}(20 \%)\end{array}$ & 0,000 & 0,000 & 8,04 & 0,00 & 0,00 \\
\hline 4 & - aumento de $I_{2} / I_{3}(20 \%)$ & 0,019 & 12,808 & 8,14 & 0,00 & 0,00 \\
\hline 5 & - aumento de $I_{1} / I_{3}(20 \%)$ & 0,019 & 10,111 & 8,27 & 0,00 & 0,00 \\
\hline 6 & - aumento de $I_{2} / I_{3}(22 \%)$ & 0,894 & 732,616 & Inestable & Inestable & Inestable \\
\hline 7 & $\begin{array}{l}\text {-aumento de } \\
\delta_{\beta}\left(\beta_{2}=0,14\right) .\end{array}$ & 0,024 & 1,515 & 8,70 & $\begin{array}{c}0,00 \\
\left(1,059 \cdot 10^{-3}\right)\end{array}$ & $\begin{array}{c}0,00 \\
\left(1,895 \cdot 10^{-3}\right)\end{array}$ \\
\hline 8 & $-k_{21}=0$ & 0,000 & 0,000 & 8,23 & 0,00 & 0,00 \\
\hline 9 & - aumento de $k_{21}(20 \%)$ & 0,024 & 20,013 & 7,97 & 0,00 & 0,00 \\
\hline 10 & $\begin{array}{l}\text {-aumento de } C_{L \alpha} / C_{D} \\
(20 \%)\end{array}$ & 0,019 & 12,800 & 8,90 & 0,00 & 0,00 \\
\hline
\end{tabular}

* Corresponde al tiempo hasta alcanzar la solución de equilibrio medido a partir que el ángulo de nutación $\theta$ entra en una banda de $\pm 0,01$ radianes al respecto de su equilibrio.

** Corresponde al ángulo de nutación alcanzado a partir de la simulación numérica. 
*** Corresponde al ángulo de nutación previsto por el modelo analítico.

En todos los casos la solución de equilibrio para $k_{31}=0$ es Tipo I.

En las variantes denominadas como 2,3 y 4 , el parámetro $k_{21}=0$, y por lo tanto $N_{e}{ }^{\prime}=0, k_{e}=$ 0 . Se puede observar que para valores idénticos de los parámetros $N_{e}, k_{e}$ se obtuvieron soluciones disímiles en cuanto a tiempo hasta alcanzar la solución de equilibrio para un mismo tipo de solución de equilibrio. Si tomamos la variante 2 se verifica que un aumento de $I_{1} / I_{3}$, a igual $I_{2} / I_{3}$, implica un aumento del tiempo para alcanzar la solución de equilibrio. La variante 3 muestra que si disminuimos $I_{2} / I_{3}$, el tiempo disminuye.

Si consideramos el Caso Base y la variante 4, vemos que una variación en el momento de inercia $I_{2}$ no produce cambios de magnitud apreciable en la respuesta dinámica del sistema. En la variante 6 un aumento mayor de $I_{2} / I_{3}$ da como resultado la inestabilidad del sistema.

La variante 5 presenta un aumento de $I_{1} / I_{3}$, que resulta en un aumento del tiempo.

La variante 7 muestra que un aumento de la diferencia de paso de las palas, $\delta_{\beta}$, con $\beta_{1}=0,07$ y $\beta_{2}=0,14$, produce un aumento en el tiempo para alcanzar el equilibrio.

Es posible observar que si aumentamos el valor de $k_{21}$, como sucede en la variante 9, disminuye el tiempo para alcanzar la solución de equilibrio.

La variante 10 muestra que si aumentamos la relación $C_{L \alpha} / C_{D}$, entonces aumenta el tiempo para alcanzar la solución de equilibrio.

En la Figura 3.58 se presentan una serie de simulaciones realizadas con el objeto de explorar distintas zonas del gráfico $N_{e}{ }^{\prime}, k_{e}$. El modelo analítico presentado por Nadal Mora (2005), y el desarrollado en este documento, prevén una zona de estabilidad definida por $N_{e}{ }^{\prime}>1$, independiente de $k_{e}$, que se muestra en la Figura 3.58. Las simulaciones numéricas muestran el comportamiento del pararrotor incluyendo efectos no lineales y efectos de órdenes de magnitud menores no contemplados en el modelo analítico. Estos efectos pueden ser importantes cerca de los límites de estabilidad, como se muestra a continuación. 
En particular, se muestra en la Figura 3.58 la existencia de tres tipos de soluciones, que incluye las denominadas Tipo II. Con el fin de determinar un límite entre soluciones Tipo I y Tipo II se consideró el error relativo entre el valor de $\theta_{e}$ hallado analíticamente y el hallado numéricamente. Se estableció como convención que son soluciones Tipo II, aquellas en donde el error relativo de $\theta_{e}$ es mayor al $50 \%$. Se puede ver que para casos donde $k_{e}>20$ existe un límite entre soluciones Tipo I y Tipo II dado por un valor aproximado de $N_{e}{ }^{\prime}=0,69$. Para valores de $k_{e}$ menores a 20 el limite se va desplazando hacia valores mayores de $N_{e}$, conforme se disminuye $k_{e}$. De la misma manera puede observase que el límite de estabilidad de las simulaciones numéricas se va desplazando hacia valores mayores de $N_{e}$ ' conforme se disminuye $k_{e}$. Este comportamiento incluye una asíntota para valores de $k_{e}=0$.

Finalmente puede observase en la Figura 3.58 una serie de casos inestables que definen una zona del gráfico de $N_{e}{ }^{\prime}, k_{e}$ de soluciones inestables.

Estas simulaciones confirman la existencia de regiones en el gráfico $N_{e}{ }^{\prime}, k_{e}$ de estabilidad predicho por el sistema analítico, evidenciando la utilidad de la utilización de los parámetros $N_{e}$, $k_{e}$ para la descripción del comportamiento dinámico del sistema y su estabilidad, lo que representa una gran economía de esfuerzo, dado elevado número de parámetros que intervienen en el problema. 


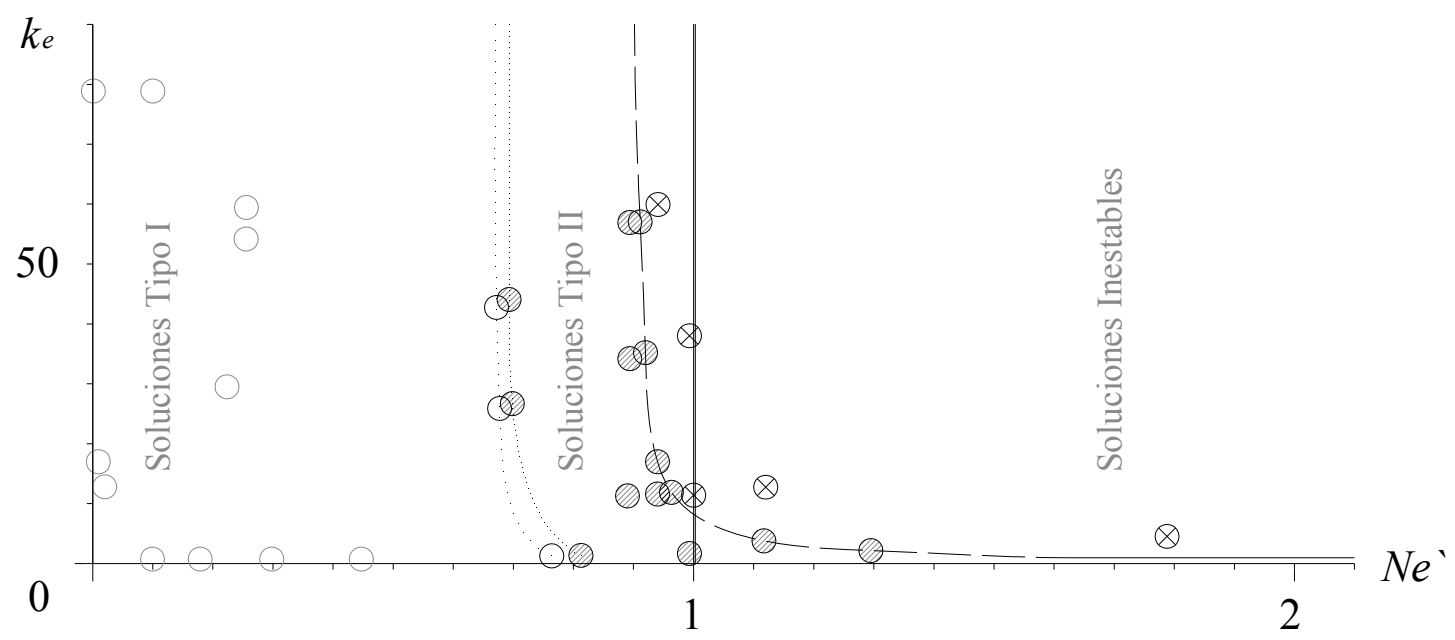

* Límite entre soluciones Tipo I y Tipo II, dado por un error relativo entre el ángulo de nutación previsto por el modelo analítico y el arrojado por la simulación del órden del $25 \%$.

Soluciones Tipo I

Soluciones Tipo II

Soluciones Inestables

Límite de estabilidad (modelo analítico)

Límite de estabilidad (modelo numérico)

Diferencia relativa del 50\% entre la solución analítica y numérica

Diferencia relativa del $20 \%$ entre la solución analítica y numérica

Figura 0.58 Regiones de estabilidad. Gráfico $N_{e}{ }^{\prime}, k_{e}$, con $k_{31}=0$.

\subsubsection{Variantes con $k_{31} \geq 0$}

En la Tabla 3.23 se presentan una selección de las variantes que fueron simuladas. En todos los casos se corroboraron las observaciones realizadas con casos accesorios no presentados. 
Tabla 0.23 Comportamiento dinámico de variantes del caso con $k_{31}>0$ y parámetros $N_{e}{ }^{\prime}, k_{e}$.

\begin{tabular}{|c|c|c|c|c|c|}
\hline $\begin{array}{l}\text { Número } \\
\text { de } \\
\text { Variante }\end{array}$ & Detalle del caso & $N_{e}$ & $\boldsymbol{k}_{e}$ & $\begin{array}{c}t_{e e}{ }^{*} \\
{[\mathbf{s}]}\end{array}$ & $\begin{array}{c}\text { Solución de } \\
\text { Equilibrio } \\
\theta_{e} \\
\text { [rad] }\end{array}$ \\
\hline 11 & $-k_{31}=0,25$ & 0,019 & 12,808 & 12,02 & 0,00 \\
\hline 12 & $\begin{array}{l}-k_{31}=0,25 \\
\text { - aumento de } I_{2} / I_{3}(20 \%)\end{array}$ & 0,107 & 157,042 & 14,82 & 0,00 \\
\hline 13 & $\begin{array}{l}-k_{31}=0,25 \\
-k_{21}=0\end{array}$ & $-0,009$ & 0,000 & 9,03 & 0,00 \\
\hline 14 & $\begin{array}{l}-k_{31}=0,25 \\
\text {-aumento de } k_{21}(20 \%)\end{array}$ & 0,014 & 18,444 & 12,19 & 0,00 \\
\hline 15 & $-k_{31}=1$ & $-0,015$ & 12,808 & 45,30 & 0,68 \\
\hline 16 & $\begin{array}{l}-k_{31}=1 \\
-k_{21}=0\end{array}$ & $-0,340$ & 0,000 & 59,80 & 0,73 \\
\hline 17 & $\begin{array}{l}-k_{31}=1 \\
- \text { aumento de } k_{21}(20 \%)\end{array}$ & $-0,011$ & 18,444 & 48,00 & 0,69 \\
\hline 18 & $\begin{array}{l}-k_{31}=1 \\
\text { - aumento de } I_{2} / I_{3}(20 \%)\end{array}$ & $-0,153$ & 157,042 & 50,70 & 0,67 \\
\hline 19 & $\begin{array}{l}-k_{31}=1 \\
\text { - aumento de } I_{1} / I_{3}(20 \%)\end{array}$ & $-0,015$ & 9,572 & 52,6 & 0,63 \\
\hline 20 & $-k_{31}=10$ & $-0,321$ & 12,808 & 9,80 & 0,80 \\
\hline 21 & $\begin{array}{l}-k_{31}=1 \\
\text { - aumento de } C_{L \alpha} / C_{D}(20 \%)\end{array}$ & $-7,053$ & 0,748 & 42,15 & 0,78 \\
\hline
\end{tabular}

* Corresponde al tiempo hasta alcanzar la solución de equilibrio medido a partir que el ángulo de nutación $\theta$ entra en una banda de $\pm 0,01$ radianes al respecto de su equilibrio.

Se puede observar en la variante 11 que un aumento pequeño de $k_{31}\left(k_{31}=0,25\right)$ produce un aumento del tiempo al respecto de la presentado Caso Base. La variante 12 muestra que si se aumenta $I_{2} / I_{3}$, también aumenta el tiempo de evolución a la solución de equilibrio al respecto de la variante anterior. 
La variante 13 muestra que si $k_{21}=0$ entonces el tiempo disminuye. La variante 14 muestra que si $k_{21}$ aumenta el tiempo aumenta. Este comportamiento resulta en sentido contrario al evidenciado para $k_{31}=0$, para pequeños valores de $k_{31}$. La variante 17 muestra que si $k_{21}=0$, con $k_{31}=1$, entonces el tiempo aumenta. La variante 18 muestra que si $k_{21}=1$, con $k_{31}=1$, el tiempo también aumenta. Este hecho implica la existencia de un valor $k_{21}$ para un tiempo mínimo.

La variante 15 indica que para un valor más grande de $k_{31}$, para los mismos parámetros definidos para el Caso Base, la solución de equilibrio es de Tipo II.

La variante 16 introduce un aumento de $k_{31}\left(k_{31}=1\right)$ que muestra un cambio de solución de equilibrio, a Tipo II, y un aumento del tiempo hasta alcanzar la solución de equilibrio.

Las variantes 18 y 19 muestran que un aumento respectivo del $20 \%$ en $I_{2} / I_{3}$ e $I_{1} / I_{3}$ producen un aumento del tiempo.

La variante 20 muestra que un aumento importante de $k_{31}, k_{31}=10$, produce una reducción de tiempo hasta alcanzar la solución de equilibrio.

La variante 21 indica que si aumentamos la relación $C_{L \alpha} / C_{D}$, disminuye el tiempo para alcanzar la solución de equilibrio.

\subsubsection{3 $\underline{\text { Variantes con } \mathrm{k} 31<0}$}

En la Tabla 3.24 se presentan una selección de las variantes que fueron simuladas. En todos los casos se corroboraron las observaciones realizadas con casos accesorios no presentados en la tabla.

Tabla 0.24 Comportamiento dinámico de variantes del caso con $k_{31}<0$ y parámetros $N_{e}$, $k_{e}$.

\begin{tabular}{|c|c|c|c|c|c|}
\hline $\begin{array}{c}\text { Número } \\
\text { de } \\
\text { Variante }\end{array}$ & Detalle del caso & $N_{e}$, & $k_{e}$ & $t_{e e}$ & $\begin{array}{c}\text { Solución de } \\
\text { Equilibrio } \\
\boldsymbol{\theta}_{e} \\
{[\mathbf{r a d}]}\end{array}$ \\
\hline
\end{tabular}




\begin{tabular}{|c|c|c|c|c|c|}
\hline $\begin{array}{l}\text { Número } \\
\text { de } \\
\text { Variante }\end{array}$ & Detalle del caso & $N_{e}$ & $\boldsymbol{k}_{e}$ & $\begin{array}{l}t_{e e} \\
{[\mathbf{s}]}\end{array}$ & $\begin{array}{c}\text { Solución de } \\
\text { Equilibrio } \\
\theta_{\boldsymbol{e}} \\
\text { [rad] }\end{array}$ \\
\hline 22 & $-k_{31}=-1$ & 0,053 & 12,808 & 27,4 & 0,00 \\
\hline 23 & $\begin{array}{l}-k_{31}=-1 \\
- \text { aumento de } I_{2} / I_{3}(20 \%)\end{array}$ & 0,542 & 157,042 & 26,1 & 0,00 \\
\hline 24 & $\begin{array}{l}-k_{31}=-1 \\
\text { - aumento de } I_{1} / I_{3}(20 \%)\end{array}$ & 0,053 & 9,5719 & 25,6 & 0,00 \\
\hline 25 & $\begin{array}{l}-k_{31}=-1 \\
\text { - aumento de } I_{2} / I_{3}(22 \%)\end{array}$ & 2,493 & 732,616 & \multicolumn{2}{|c|}{ Solución inestable } \\
\hline 26 & $\begin{array}{l}-k_{31}=-1 \\
-k_{21}=0\end{array}$ & 0,034 & 0,000 & 289 & 0,29 \\
\hline 27 & $\begin{array}{l}-k_{31}=-1 \\
\text { - aumento de } k_{21}(20 \%)\end{array}$ & 0,057 & 18,444 & 19,7 & 0,00 \\
\hline 28 & $\begin{array}{l}k_{31}=-1, \text { aumento de } C_{L \alpha} / C_{D} \\
(20 \%)\end{array}$ & 0,053 & 12,808 & 27,7 & 0,00 \\
\hline
\end{tabular}

En la primera simulación de este conjunto, variante 22, es posible observar que el tiempo para alcanzar la solución de equilibrio se incrementa respecto del Caso Base. No obstante este tiempo resulta menor que la variante $14\left(\operatorname{con} k_{31}=1\right)$.

Las variantes 23 y 24 muestran que si se aumenta respectivamente $I_{1} / I_{3}$ e $I_{2} / I_{3}$, el tiempo disminuye. Si se aumenta $I_{2} / I_{3}$ un $22 \%$ la solución resulta inestable como se aprecia en la variante 25 .

Si $k_{21}=0$, la solución de equilibrio es de Tipo II, y el tiempo para alcanzar dicha solución resulta notablemente mayor, como se ve en la variante 26. Por otro lado si se aumenta un 20 $\%$ el valor de $k_{21}$ al respecto de Caso Base (variante 27) la solución es de Tipo I, el tiempo es menor al correspondiente con la variante 21 .

La simulación de la variante 28 indica que el aumento del parámetro $C_{L \alpha} / C_{D}$ lleva a un aumento del tiempo para llegar a la solución de equilibrio. 


\subsubsection{Conclusiones}

Se puede concluir a partir de la observación de las simulaciones anteriores que para $I_{3}>I_{2}, I_{1}$, es posible obtener 3 tipos de soluciones. Las denominadas Tipo I, II, e inestabilidad.

Cuando el centro de masas se encuentra en el plano que contiene a las palas, $k_{31}=0$, se observa que en el plano $N_{e}$, $k_{e}$ quedan definidos dos límites: aquel que separa las soluciones Tipo I y Tipo II, y aquel que separa las soluciones Tipo II de las soluciones inestables. Para valores de $k_{e}$ mayores a 20 se observa un comportamiento en términos de tipo de solución aproximadamente constante con $k_{e}$.

De acuerdo al modelo analítico presentado en el capítulo anterior, en el presente caso, donde $I_{3}>I_{2}, I_{1}$, es accesible el primer y segundo cuadrante del plano $\left(N_{e}{ }^{\prime}, k_{e}\right)$. La región que garantiza la estabilidad, de acuerdo a este modelo analítico, es $N_{e}{ }^{\prime}<1$ (tal cual se indica en la Figura 2.8).

El aumento de $k_{31}$ tiene dos efectos posibles de acuerdo a su magnitud y a la configuración geométrica y aerodinámica del pararrotor: uno de los efectos es el aumento del tiempo de respuesta al respecto de un caso idéntico con $k_{31}=0$, y el otro es el corrimiento de los límites entre tipo de soluciones en el plano $N_{e}{ }^{\prime}, k_{e}$ (Piechocki, 2010). En cualquier caso un aumento $k_{31}$ tiende a disminuir la sensibilidad del pararrotor a factores desestabilizantes y presenta un efecto estabilizante. Por otro lado un aumento significativo de la magnitud $k_{31}$, pero con signo negativo, presenta en un gran número de casos simulados una solución inestable, donde el pararrotor se invierte y $k_{31}$ pasa a tener signo positivo.

Al respecto del efecto de $k_{21}$, si $k_{31}=0$, su aumento implica una disminución del tiempo para alcanzar la solución de equilibrio. En el caso que $k_{31}>0$, cuando la solución es de Tipo I, existe un valor de $k_{21}$ que hace mínimo el tiempo hasta alcanzar la solución de equilibrio, en torno al cual cualquier cambio en este parámetro produce un aumento del tiempo. Si $k_{31}<0$ un aumento de $k_{21}$ hace reducir el tiempo hasta alcanzar la solución de equilibrio. Una disminución por otro lado promueve soluciones Tipo II, con un aumento considerable del tiempo. 
$\mathrm{Al}$ respecto de los parámetros $I_{1} / I_{3}, I_{2} / I_{3}$ con $I_{3}>I_{2}>I_{1}$, se puede observar que para valores de $k_{31}>0$ y $k_{31}=0$, su aumento tiene como consecuencia un aumento de tiempo para alcanzar la solución de equilibrio, y dependiendo de la magnitud del aumento la introducción de soluciones Tipo II, o la inestabilidad del sistema. Cuando $k_{31}<0$ el efecto de este aumento es la disminución del tiempo. Dependiendo de la magnitud puede llevar a la inestabilidad del sistema.

Al respecto del parámetro $C_{L \alpha} / C_{D}$ se puede ver que su aumento trae como consecuencia una disminución del tiempo para $k_{31}=0$ y $k_{31}>0$, y un aumento para $k_{31}<0$.

En la Tabla 3.25 se presentan resumidamente las conclusiones a las que se arriba.

Tabla 0.25 Efecto en el comportamiento dinámico del pararrotor, en el tiempo de establecimiento de la solución de equilibrio, $t_{e e}$, tomando como referencia el Caso Base, de los parámetros $k_{31,} k_{21}, I_{1} / I_{3}, C_{L \alpha} / C_{D}$.

\begin{tabular}{|c|l|l|l|l|l|}
\hline $\boldsymbol{k}_{\mathbf{3 1}}$ & $\boldsymbol{k}_{\mathbf{2 1}}$ & $\boldsymbol{I}_{\mathbf{1}} / \boldsymbol{I}_{\mathbf{3}}$ & $\boldsymbol{I}_{\mathbf{2}} / \boldsymbol{I}_{\mathbf{3}}$ & $\boldsymbol{C}_{\boldsymbol{L} \boldsymbol{\alpha}} / \boldsymbol{C}_{\boldsymbol{D}}$ & \multicolumn{1}{|c|}{ Resultado } \\
\hline 0 & $+20 \%$ & & & & Disminuye $t_{e e}$ \\
\hline 0 & $=0$ & & & & Aumenta $t_{e e}$ \\
\hline 0 & & $+20 \%$ & & & Aumenta $t_{e e}$ \\
\hline 0 & & & $+20 \%$ & & No se registran cambios \\
\hline 0 & & & $+22 \%$ & & Solución Inestable \\
\hline 0 & & & & $+20 \%$ & Aumenta el $t_{e e}$ \\
\hline 0 & & & & & Aumenta el $t_{e e}$ \\
\hline
\end{tabular}




\begin{tabular}{|c|c|c|c|c|c|}
\hline$k_{31}$ & $k_{21}$ & $I_{1} / I_{3}$ & $I_{2} / I_{3}$ & $C_{L \alpha} / C_{D}$ & Resultado \\
\hline$>0$ & $+20 \%$ & & & & Aumenta el $t_{e e}$ \\
\hline$>0$ & $=0$ & & & & $\begin{array}{l}\text { Aumenta o disminuye el } t_{e e} \text { en función de la } \\
\text { magnitud de } k_{31} \text { registrándose en algún caso un } \\
\text { mínimo absoluto }\end{array}$ \\
\hline$>0$ & & $+20 \%$ & & & Disminuye el $t_{e e}$ \\
\hline$>0$ & & & $+20 \%$ & & Aumenta $t_{e e}$ \\
\hline$>0$ & & & & $+20 \%$ & $\begin{array}{l}\text { Aumenta } t_{e e} \text { y el ángulo de nutación en caso de } \\
\text { soluciones Tipo II }\end{array}$ \\
\hline$<0$ & & & & & Aumenta $t_{e e}$ \\
\hline$<0$ & $+20 \%$ & & & & Disminuye $t_{e e}$ \\
\hline$<0$ & $=0$ & & & & Aumenta $t_{e e}$ \\
\hline$<0$ & & $+20 \%$ & & & Disminuye $t_{e e}$ \\
\hline$<0$ & & & $+20 \%$ & & $\begin{array}{l}\text { Aumenta } t_{e e} \text { hasta la inestabilidad del sistema } \\
\text { en función de la magnitud }\end{array}$ \\
\hline$<0$ & & & & $+20 \%$ & Aumenta $t_{e e}$ \\
\hline
\end{tabular}

\subsubsection{Caso B - Eje principal intermedio próximo al eje de giro. $I_{2}>I_{3}>I_{1}$.}

El presente caso se basa en el cumplimiento de la condición que $I_{2}>I_{3}>I_{1}$. Se adoptarán los siguientes momentos de inercia, presentados en la Tabla 3.26. 
Tabla 0.26 Momentos de inercia para el caso $I_{2}>I_{3}>I_{1}$.

\begin{tabular}{|c|c|}
\hline \multicolumn{2}{|c|}{ Momentos de inercia } \\
\hline$I_{1}$ & $6 \cdot 10^{-4} \mathrm{~kg} \mathrm{~m}^{2}$ \\
\hline$I_{2}$ & $22 \cdot 10^{-4} \mathrm{~kg} \mathrm{~m}^{2}$ \\
\hline$I_{3}$ & $16 \cdot 10^{-4} \mathrm{~kg} \mathrm{~m}^{2}$ \\
\hline
\end{tabular}

Este caso presenta una condición de inestabilidad en la simulación numérica, como se puede ver en la Figura 3.59.

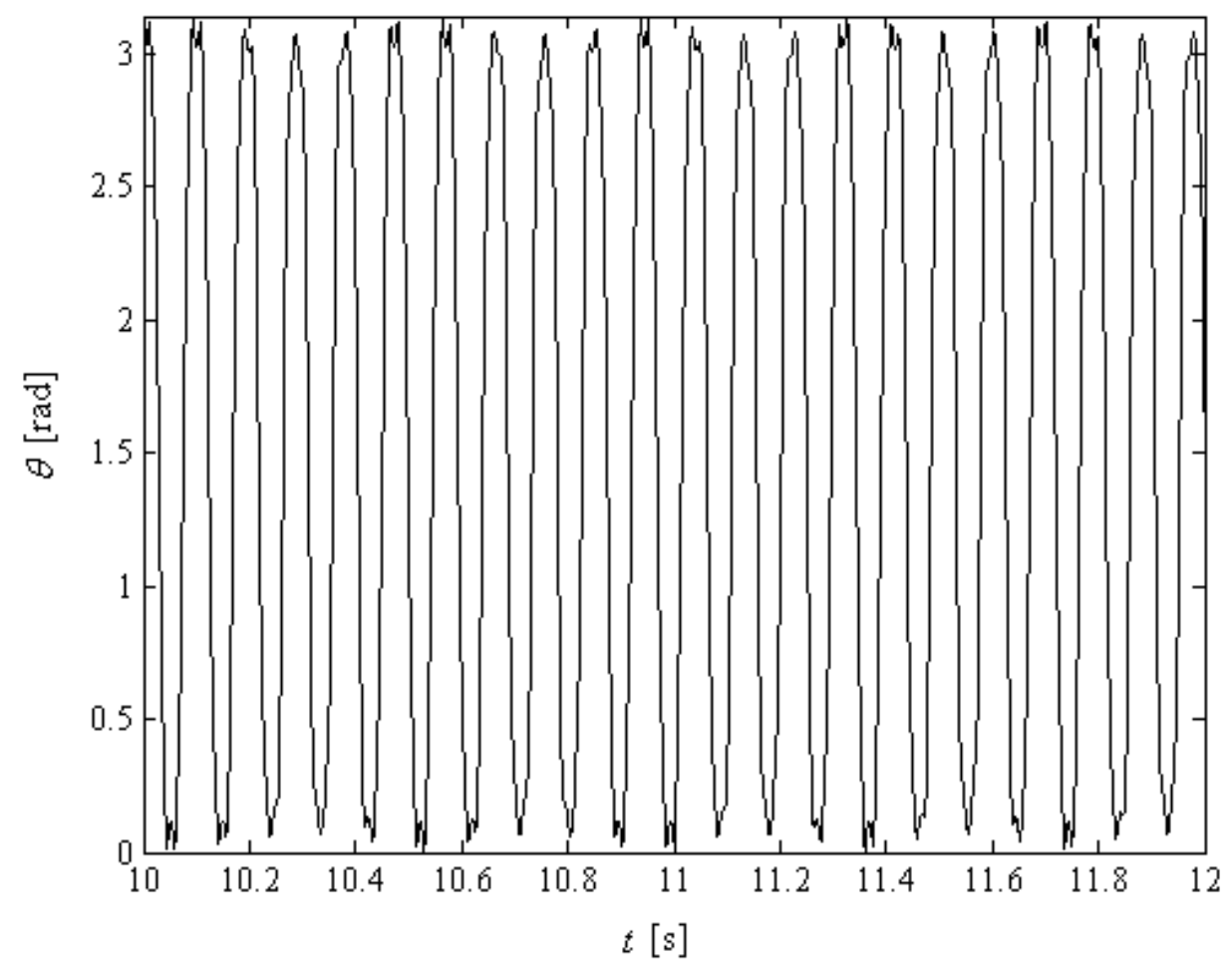

Figura 0.59 Evolución del ángulo de nutación, $\theta$, con el tiempo, $t$, para $k_{31}=0$ entre los 10 segundos y 12 segundos de simulación, para un dominio entre 0 y $\pi$. 
Se evidencia que el efecto del parámetro $k_{31}$ no resulta relevante para esta configuración de momentos de inercia, por cuanto no se observa efecto en la estabilidad del sistema.

El modelo analítico aplicado al presente caso numérico predice una respuesta de inestabilidad dinámica para este caso. La solución predicha por el modelo analítico es de sillas inestables basada en autovalores reales positivos que se muestran en la Tabla 3.27.

Tabla 0.27 Parte real de los autovalores de este caso de acuerdo al modelo analítico.

\begin{tabular}{|c|c|}
\hline \multicolumn{2}{|c|}{$\begin{array}{c}\text { Parte real de los autovalores } \\
\text { (modelo analítico) }\end{array}$} \\
\hline$k_{31}=1$ & 0,6346 \\
\hline$k_{31}=0,5$ & 0,6416 \\
\hline$k_{31}=0$ & 0,6468 \\
\hline$k_{31}=-0,5$ & 0,6502 \\
\hline$k_{31}=-1$ & 0,6517 \\
\hline
\end{tabular}

El comportamiento dinámico de distintas variantes del presente caso muestra que la inestabilidad introducida por la configuración de los momentos de inercia es insensible al respecto de otros parámetros geométricos y aerodinámicos, y en particular de $k_{31}$. Se ha observado que para valores extremadamente grandes de $k_{31}\left(k_{31}=100\right.$, que resulta impracticable desde el punto de vista técnico) es posible alcanzar soluciones estables, coincidentemente con la predicción del modelo analítico a partir del cálculo de autovalores (Chi-Tsong Chen, 1999). En la Tabla 3.28 se observan las variantes del caso presente.

Tabla 0.28 Comportamiento dinámico de variantes del caso y parámetros $N_{e}{ }^{\prime}, k_{e}$.

\begin{tabular}{|l|l|l|}
\hline$k_{31}$ & Modelo analítico & Simulación \\
\hline
\end{tabular}




\begin{tabular}{|c|c|c|c|c|c|}
\hline & $\begin{array}{c}\lambda_{1} \text { (parte } \\
\text { real) }\end{array}$ & $\lambda_{2}$ (parte real) & $\boldsymbol{N}_{\boldsymbol{e}}{ }^{\prime}$ & $\boldsymbol{k}_{\boldsymbol{e}}$ & Solución de equilibrio \\
\hline-1 & 0,651 & $-0,669$ & $-0,04$ & $-4,211$ & Inestable \\
\hline$-0,5$ & 0,650 & $-0,662$ & $-0,03$ & $-4,211$ & Inestable \\
\hline 0 & 0,647 & $-0,657$ & $-0,015$ & $-4,211$ & Inestable \\
\hline 0,5 & 0,642 & $-0,658$ & $-0,002$ & $-4,211$ & Inestable \\
\hline 1 & 0,635 & $-0,652$ & 0,012 & $-4,211$ & Inestable \\
\hline 1 & 0,834 & $-0,849$ & 0,0114 & 0,000 & $\begin{array}{c}31,2 \text { segundos para una } \\
\text { solución Tipo II }\end{array}$ \\
\hline 1 & $-0,0005$ & $-0,017$ & 1,41 & $-1,51$ & $\begin{array}{c}2,54 \text { segundos para una } \\
\text { solución Tipo II }\end{array}$ \\
\hline
\end{tabular}

De acuerdo al modelo analítico presentado en el capítulo anterior, en el presente caso, donde $I_{2}>I_{3}>I_{1}$, son accesibles el tercer y cuarto cuadrante del plano $\left(N_{e}, k_{e}\right)$. La región que garantiza la estabilidad de acuerdo al modelo analítico es $N_{e}{ }^{\prime}>1$ (tal cual se indica en la Figura 2.9). Se puede observar en las simulaciones que los casos de inestabilidad y estabilidad se condicen con lo que predice el modelo analítico.

\subsubsection{Caso C - Eje principal intermedio próximo al eje de giro. $I_{1}>I_{3}>I_{2}$.}

El presente caso se basa en el cumplimiento de la condición que $I_{1}>I_{3}>I_{2}$. Por tanto se adoptarán los momentos de inercia presentados en la Tabla 3.29.

Tabla 0.29 Momentos de inercia para el caso $I_{1}>I_{3}>I_{2}$.

\begin{tabular}{|c|c|}
\hline \multicolumn{2}{|c|}{ Momentos de inercia } \\
\hline$I_{1}$ & $22 \cdot 10^{-6} \mathrm{~kg} \mathrm{~m}^{2}$ \\
\hline$I_{2}$ & $6 \cdot 10^{-6} \mathrm{~kg} \mathrm{~m}^{2}$ \\
\hline
\end{tabular}




\begin{tabular}{|c|c|}
\hline \multicolumn{2}{|c|}{ Momentos de inercia } \\
\hline$I_{3}$ & $16 \cdot 10^{-6} \mathrm{~kg} \mathrm{~m}^{2}$ \\
\hline
\end{tabular}

Una respuesta dinámica típica de los casos simulados se presenta en la Figura 3.60.

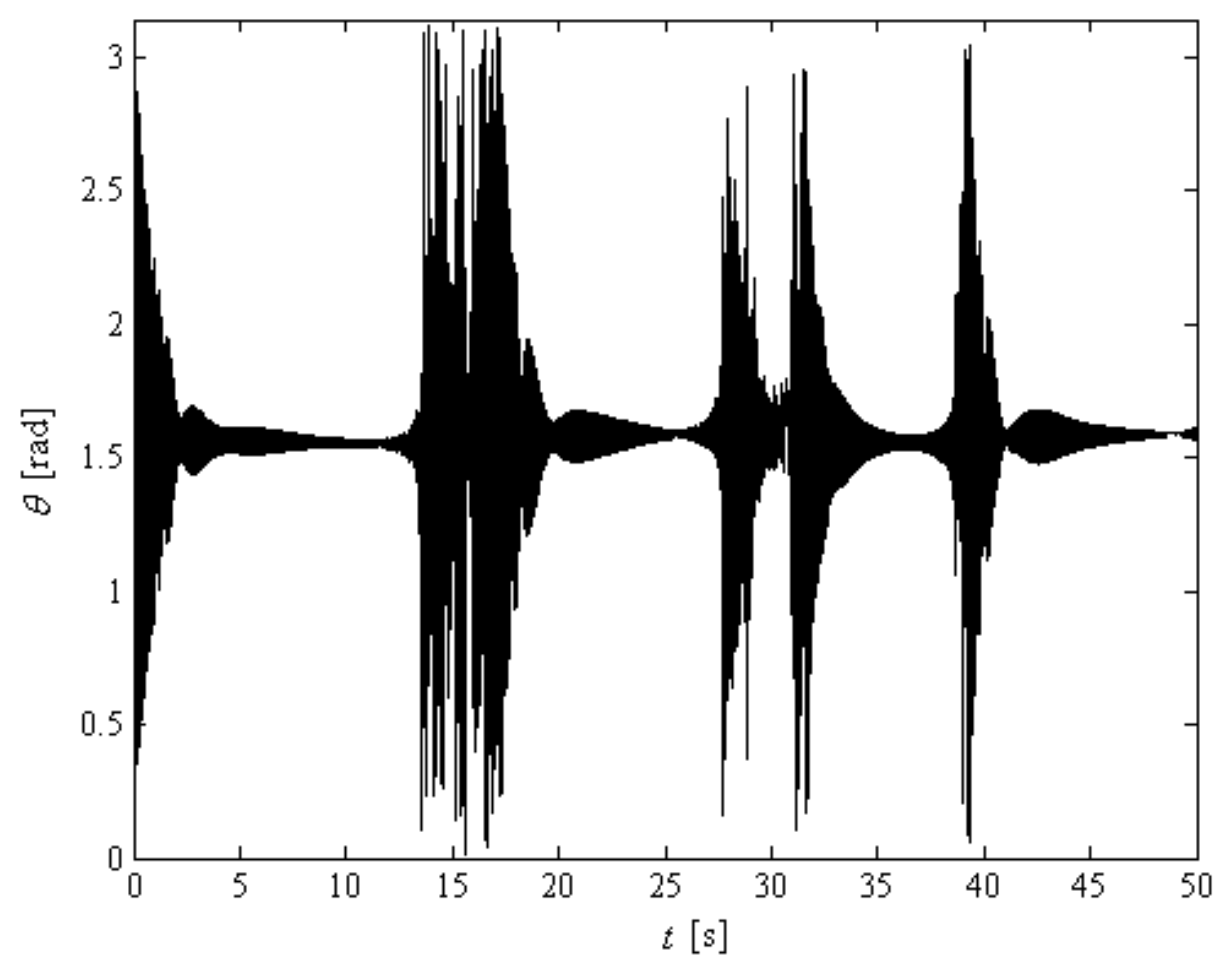

Figura 0.60 Evolución del ángulo de nutación, $\theta$ con el tiempo, $t$, para $k_{31}=0$, para un dominio entre 0 y $\pi$.

Este caso presenta una condición de inestabilidad en la simulación numérica determinada por los momentos de inercia, como se aprecia en la Figura 3.61. El efecto en este comportamiento del parámetro $k_{31}$ resulta irrelevante. No obstante, si se simula el caso incorporando grandes valores de $k_{31}$, es posible obtener soluciones estables como se muestra en la Tabla 3.30.

En la Tabla 3.30 se muestran distintas variantes para el presente caso, se presentan los valores de la parte real de los autovalores del modelo analítico, y el valor de los parámetros $N_{e}{ }^{\prime}, k_{e}$. 
Puede observarse que el comportamiento dinámico que predice el modelo analítico a partir del cálculo de autovalores difiere del obtenido en la simulación para el caso donde $k_{31}$ es de gran magnitud, $k_{31}=10$, como resulta esperar a partir de las hipótesis.

Tabla 0.30 Comportamiento dinámico de variantes del caso y parámetros $N_{e}$, $k_{e}$.

\begin{tabular}{|c|c|c|c|c|c|}
\hline \multirow{2}{*}{$\boldsymbol{k}_{\mathbf{3 1}}$} & \multicolumn{4}{|c|}{ Modelo analítico } & Simulación \\
\cline { 2 - 6 } & $\lambda_{\mathbf{1}}$ (parte real) & $\lambda_{\mathbf{2}}$ (parte real) & $\boldsymbol{N}_{\boldsymbol{e}}{ }^{\prime}$ & $\boldsymbol{k}_{\boldsymbol{e}}$ & Solución de equilibrio \\
\hline-1 & 4,640 & $-0,686$ & 0,025 & $-0,122$ & Inestable \\
\hline 0 & 4,640 & $-0,690$ & 0,009 & $-0,122$ & Inestable \\
\hline 1 & 4,640 & $-0,697$ & $-0,007$ & $-0,122$ & Inestable \\
\hline 10 & 0,612 & $-0,855$ & $-0,151$ & $-0,122$ & $\begin{array}{c}2,17 \text { segundos para una } \\
\text { solución Tipo II (0,19 rad) }\end{array}$ \\
\hline
\end{tabular}

De acuerdo al modelo analítico presentado en el capítulo anterior, en el presente caso, donde $I_{1}>I_{3}>I_{2}$, son accesibles el tercer y cuarto cuadrante del plano $\left(N_{e}{ }^{\prime}, k_{e}\right)$.

\subsubsection{Caso D - Eje principal menor próximo al eje de giro.}

El presente caso se basa en el cumplimiento de la condición que $I_{3}<I_{2}<I_{1}$. Para el cumplimiento de la condición se adoptan los momentos de inercia que se presentan en la Tabla 3.31 . 
Tabla 0.31 Momentos de inercia para $I_{3}<I_{2}, I_{1}$.

\begin{tabular}{|c|c|}
\hline \multicolumn{2}{|c|}{ Momentos de inercia } \\
\hline$I_{1}$ & $21 \cdot 10^{-6} \mathrm{~kg} \mathrm{~m}^{2}$ \\
\hline$I_{2}$ & $23 \cdot 10^{-6} \mathrm{~kg} \mathrm{~m}^{2}$ \\
\hline$I_{3}$ & $15 \cdot 10^{-6} \mathrm{~kg} \mathrm{~m}^{2}$ \\
\hline
\end{tabular}

Las condiciones de equilibrio para una condición de $\beta_{1}=0,07 \mathrm{rad}$ y $\beta_{2}=0,07 \mathrm{rad}$ de acuerdo al modelo analítico para el presente caso numérico son:

$-\omega_{e q 1}=0$

$-\omega_{e q 2}=0$

Los valores resultado de la simulación numérica para idénticos valores de configuración del modelo muestran resultados coincidentes. El ángulo de nutación de equilibrio para esta condición es cero.

Para observar el efecto del parámetro $k_{31}$ en el comportamiento dinámico del pararrotor se consideró un ángulo de nutación inicial de 0,1 radianes, y se observó la evolución del ángulo de nutación. Se computó el tiempo transcurrido desde la posición inicial del pararrotor hasta que se alcanza el equilibrio en una banda de $\pm 0,01$ radianes.

Con el fin de observar el efecto de $k_{31}$ se simuló la dinámica de vuelo del pararrotor con momentos de inercia según la Tabla 3.30, las condiciones de base mencionadas anteriormente, y un ángulo de paso de las palas de $\beta_{1}=0,07$ y $\beta_{2}=0,07$, con distintas posiciones del centro de masas. Los resultados, en términos de la evolución del ángulo de nutación se muestran en la Figura 3.61.

La Figura 3.58 muestra, cualitativamente, la existencia de tres tipos de solución, de idénticas características a las soluciones Tipo I y Tipo II que se describieron para el caso que $I_{3}>I_{2}, I_{1}$. 
Por otro lado, la Figura 3.61 muestra que a valores positivos de $k_{31}$ la solución pasa de una Tipo I a una Tipo II, donde el ángulo de nutación disminuye con el distanciamiento del centro de masas al plano de las palas. Al respecto del tiempo hasta alcanzar la solución de equilibrio se observa un aumento del tiempo al aumentar $k_{31}$ cuando se trata de soluciones Tipo I, y una disminución del tiempo cuando las soluciones son del Tipo II. De la misma forma, para valores negativos de $k_{31}$, se puede apreciar que para valores absolutos pequeños la solución es Tipo I, y el tiempo baja para alcanzar la solución, y para mayores valores absolutos se pasa a soluciones Tipo II, cuyo ángulo de nutación crece con $k_{31}$, con una disminución de los tiempos de respuesta.

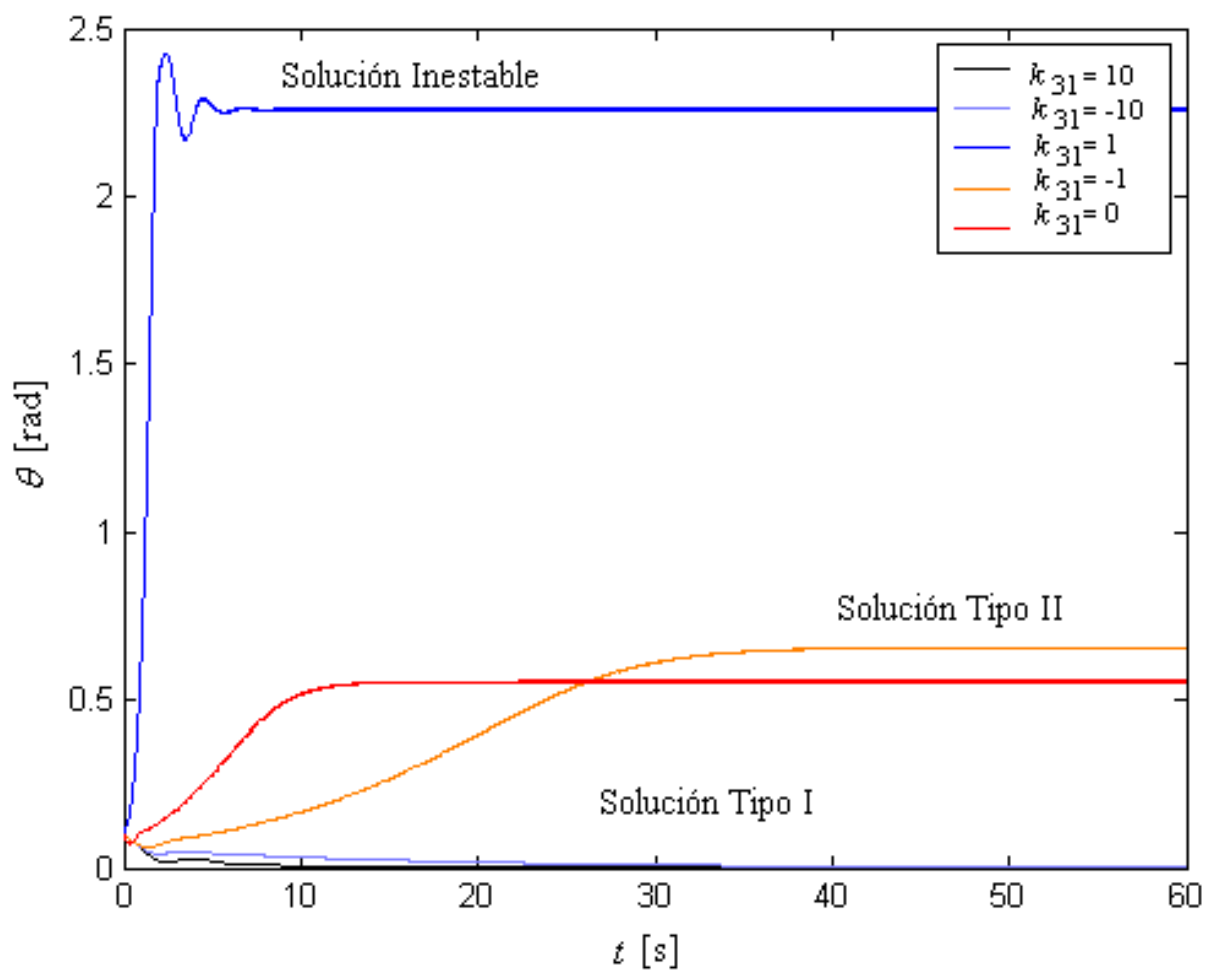

Figura 0.61 Evolución del ángulo de nutación, $\theta$, con el tiempo $t$, para distintos valores de $k_{31}$. Tipos de soluciones Tipo I, Tipo II, y soluciones inestables. 
Sobre la base de la configuración presentada (a partir de ahora considerada como Caso Base) se consideraron una serie de variantes con el fin de observar la influencia del cambio de los parámetros $k_{31}$ y $k_{21}$ asociados a la posición de las palas al respecto del centro de masas, la relación de momentos de inercia $I_{3} / I_{2}, I_{3} / I_{1}$ (sobre hipótesis que $I_{1} \geq I_{2}>I_{3}$ ), y la relación $C_{L \alpha} / C_{D}$ de las palas. A los fines de simplificar la presentación de las variantes se las dividió según el valor del parámetro $k_{31}$.

\subsubsection{Variantes con $k_{31}=0$}

En la Tabla 3.32 se presentan una selección de las variantes que fueron simuladas. En todos los casos de corroboraron las observaciones realizadas con simulaciones accesorias que no se presentan.

Tabla 0.32 Comportamiento dinámico de variantes del caso con $k_{31}=0$ y parámetros $N_{e}{ }^{\prime}, k_{e}$.

\begin{tabular}{|c|l|c|c|c|c|}
\hline $\begin{array}{c}\text { Número } \\
\text { de } \\
\text { Variante }\end{array}$ & \multicolumn{1}{|c|}{ Detalle del caso } & $\boldsymbol{N}_{\boldsymbol{e}}$ & $\boldsymbol{k}_{\boldsymbol{e}}$ & $\begin{array}{c}\boldsymbol{t}_{\boldsymbol{e}} \\
{[\mathbf{s}]}\end{array}$ & $\begin{array}{c}\text { Solución de } \\
\text { Equilibrio } \\
\boldsymbol{\theta}_{\boldsymbol{e}} \\
{[\mathbf{r a d}]}\end{array}$ \\
\hline 1 & Caso Base & $-0,0112$ & 0,6144 & 6,63 & 0,00 \\
\hline 2 & -aumento de $I_{2} / I_{3}(20 \%)$ & $-0,0072$ & 0,4702 & 6,64 & 0,00 \\
\hline 3 & - aumento de $I_{1} / I_{3}(20 \%)$ & $-0,0112$ & 0,8704 & 6,62 & 0,00 \\
\hline 4 & $-k_{21}=0$ & 0,0000 & 0,0000 & 6,34 & 0,00 \\
\hline 5 & - aumento de $k_{21}(20 \%)$ & $-0,0134$ & 0,6144 & 7,92 & 0,00 \\
\hline 6 & -aumento de $C_{L \alpha} / C_{D}(20 \%)$ & $-0,0112$ & 0,6144 & 8,80 & 0,00 \\
\hline
\end{tabular}

En todos los casos la solución de equilibrio para $k_{31}=0$ es Tipo I. 
Las variantes denominadas como 2 y 3 muestran que el aumento de $I_{2} / I_{3}$ supone un aumento en el tiempo hasta alcanzar la solución y a la inversa el aumento de $I_{1} / I_{3}$. La variante 4 muestra que si el $k_{21}=0$ el tiempo disminuye, y la variante 5 que si $k_{21}$ es un $20 \%$ mayor el tiempo aumenta.

La variante 6 indica que un aumento de $C_{L \alpha} / C_{D}$ produce un aumento del tiempo hasta alcanzar la solución.

\subsubsection{Variantes con $k_{\underline{31}} \geq 0$}

En la Tabla 3.33 se presentan una selección de las variantes que fueron simuladas. En todos los casos se corroboraron las observaciones realizadas con casos accesorios que no son presentados.

Tabla 0.33 Comportamiento dinámico de variantes del caso con $k_{31}>0$ y parámetros $N_{e}{ }^{\prime}, k_{e}$.

\begin{tabular}{|c|l|c|c|c|c|}
\hline $\begin{array}{c}\text { Número } \\
\text { de } \\
\text { Variante }\end{array}$ & \multicolumn{1}{|c|}{ Detalle del caso } & $\boldsymbol{N}_{\boldsymbol{e}}$, & $\boldsymbol{k}_{\boldsymbol{e}}$ & $\begin{array}{c}\boldsymbol{t}_{\boldsymbol{e}} \\
{[\mathbf{s}]}\end{array}$ & $\begin{array}{c}\text { Solución de } \\
\text { Equilibrio } \\
\boldsymbol{\theta}_{\boldsymbol{e}} \\
{[\mathbf{r a d}]}\end{array}$ \\
\hline 7 & $-k_{31}=1$ & 0,0088 & 0,6144 & 32,5 & 0,73 \\
\hline 8 & $\begin{array}{l}-k_{31}=1 \\
- \text { aumento de } I_{2} / I_{3}(20 \%)\end{array}$ & 0,0056 & 0,4681 & 32,3 & 0,73 \\
\hline 9 & $\begin{array}{l}-k_{31}=1 \\
- \text { aumento de } I_{1} / I_{3}(20 \%)\end{array}$ & 0,0088 & 0,8704 & 32,6 & 0,73 \\
\hline 10 & $\begin{array}{l}-k_{31}=1 \\
-k_{21}=0\end{array}$ & 0,0000 & 0,0200 & 59,2 & 0,58 \\
\hline 11 & $\begin{array}{l}-k_{31}=1 \\
- \text { aumento de } k_{21}(20 \%)\end{array}$ & 0,0066 & 0,8848 & 30,6 & 0,73 \\
\hline 12 & $\begin{array}{l}-k_{31}=1 \\
- \text { aumento de } C_{L \alpha} / C_{D}(20 \%)\end{array}$ & 0,0088 & 0,6144 & 37,4 & 0,80 \\
\hline
\end{tabular}


Se puede observar en la variante 7 que un aumento de $k_{31}\left(k_{31}=1\right)$ sobre el caso base introduce una solución de equilibrio Tipo II.

Las variantes denominadas como 8 y 9 muestran que el aumento de $I_{2} / I_{3}$ supone un aumento del tiempo hasta alcanzar la solución y a la inversa el aumento de $I_{1} / I_{3}$.

La variante 10 muestra que si $k_{21}=0$ el tiempo aumenta y disminuye el ángulo de nutación de equilibrio. La variante 11 muestra que a la inversa, si $k_{21}$ es un $20 \%$ mayor el tiempo disminuye y el ángulo aumenta.

La variante 12 indica que un aumento de $C_{L \alpha} / C_{D}$ produce un aumento del tiempo hasta alcanzar la solución y del ángulo de nutación de equilibrio.

\section{Variantes con $\boldsymbol{k}_{31}<0$}

En la Tabla 3.34 se presentan una selección de las variantes que fueron simuladas. En todos los casos se corroboraron las observaciones realizadas con casos accesorios que no se presentan en la tabla.

Tabla 0.34 Comportamiento dinámico de variantes del caso con $k_{31}<0$ y parámetros $N_{e}$, $k_{e}$.

\begin{tabular}{|c|c|c|c|c|c|}
\hline $\begin{array}{c}\text { Número } \\
\text { de } \\
\text { Variante }\end{array}$ & Detalle del caso & $N_{e}$, & $\boldsymbol{k}_{e}$ & $\begin{array}{l}t_{e e} \\
{[\mathbf{s}]}\end{array}$ & $\begin{array}{c}\text { Solución de } \\
\text { Equilibrio } \\
\theta_{e} \\
\text { [rad] } \\
\end{array}$ \\
\hline 13 & $-k_{31}=-1$ & $-0,0312$ & 0,6144 & 28,8 & 0,00 \\
\hline 14 & $\begin{array}{l}-k_{31}=-1 \\
- \text { aumento de } I_{2} / I_{3}(20 \%)\end{array}$ & $-0,0198$ & 0,4681 & 28,6 & 0,00 \\
\hline 15 & $\begin{array}{l}-k_{31}=-1 \\
\text { - aumento de } I_{1} / I_{3}(20 \%)\end{array}$ & $-0,0312$ & 0,8704 & 29,0 & 0,00 \\
\hline
\end{tabular}




\begin{tabular}{|c|l|c|c|c|c|}
\hline 16 & $\begin{array}{l}-k_{31}=-1 \\
-k_{21}=0\end{array}$ & $-0,0200$ & 0,0000 & 57,1 & 0,56 \\
\hline 17 & $\begin{array}{l}-k_{31}=-1 \\
- \text { aumento de } k_{21}(20 \%)\end{array}$ & $-0,0334$ & 0,8848 & 17,8 & 0,00 \\
\hline 18 & $\begin{array}{l}-k_{31}=-1 \\
- \text { aumento de } C_{L \alpha} / C_{D}(20 \%)\end{array}$ & $-0,0334$ & 0,8848 & 37,5 & 0,00 \\
\hline
\end{tabular}

La variante 13 muestra que existe un aumento del tiempo hasta alcanzar la solución de equilibrio al respecto de la variante del Caso Base, al disminuir $k_{31}$, para este caso $k_{31}=-1$.

Las variantes denominadas como 14 y 15 muestran que el aumento de $I_{2} / I_{3}$ supone una disminución del tiempo hasta alcanzar la solución y a la inversa el aumento de $I_{1} / I_{3}$.

La variante 16 muestra que si el $k_{21}=0$ el tiempo aumenta y la solución de equilibrio es de Tipo II. La variante 17 muestra que, si $k_{21}$ es un 20\% mayor el tiempo disminuye y la solución es Tipo I.

La variante 18 indica que un aumento de $C_{L \alpha} / C_{D}$ produce un aumento del tiempo hasta alcanzar la solución y del ángulo de nutación de equilibrio.

\subsubsection{Conclusiones}

Se puede concluir a partir de la observación de las simulaciones anteriores que para $I_{3}<I_{2}<$ $I_{1}$, es posible obtener 3 tipos de soluciones: la denominada Tipo I, II, y aquellas inestables.

En la Tabla 3.35 se resumen las conclusiones al respecto del efecto de los parámetros $k_{31}, k_{21}$, $I_{1} / I_{3}, C_{L \alpha} / C_{D}$ en la estabilidad del sistema.

El aumento de $k_{31}$ tiene dos efectos posibles de acuerdo a su magnitud y a la configuración geométrica y aerodinámica del pararrotor: uno de los efectos es el aumento del tiempo de respuesta al respecto de un caso idéntico con $k_{31}=0$, y el otro es el cambio de solución a la Tipo II. En cualquier caso un aumento significativo de $k_{31}$ tiende a disminuir la sensibilidad del pararrotor a factores desestabilizantes y presenta un efecto estabilizante. 
$\mathrm{Al}$ respecto del efecto de $k_{21}$, si $k_{31}=0$ su aumento implica un aumento en el tiempo para alcanzar la solución de equilibrio y una disminución del parámetro tiene asociada una disminución de ese tiempo hasta el límite dado por $k_{21}=0$. En el caso que $k_{31}>0$, y la solución es de Tipo II, se observa que un aumento de $k_{21}$ supone una disminución del tiempo. Si $k_{31}<0$, un aumento de $k_{21}$, hace reducir el tiempo hasta alcanzar la solución de equilibrio.

El aumento de $I_{1} / I_{3}, I_{2} / I_{3}$ con $k_{31}=0$ trae aparejado una disminución del tiempo hasta alcanzar la solución de equilibrio. A la inversa un aumento de $I_{1} / I_{3}$ con $k_{31}=0$ produce un aumento del tiempo en alcanzar la solución de equilibrio que puede llegar hasta la inestabilidad. Si se desplaza el centro de masas de forma que $k_{31}>0$, un aumento de $I_{1} / I_{3}, I_{2} /$ $I_{3}$ trae aparejado un aumento del tiempo hasta la solución de equilibrio. El límite para el incremento de este parámetro es la inestabilidad. Por el contrario un aumento de $I_{1} / I_{3}$ disminuye el tiempo para alcanzar la solución de equilibrio. Si se desplaza el centro de masas de forma que $k_{31}<0$, un aumento de $I_{1} / I_{3}$ implica un aumento del tiempo y a la inversa cuando se aumenta $I_{2} / I_{3}$.

Al respecto del parámetro $C_{L \alpha} / C_{D}$ se puede ver que su aumento trae como consecuencia un aumento del tiempo para $k_{31}=0, k_{31}<0$ y $k_{31}>0$.

Tabla 0.35 Efecto en el comportamiento dinámico del pararrotor, tomando como referencia el Caso Base, de los parámetros $k_{31}, k_{21}, I_{1} / I_{3}, C_{L \alpha} / C_{D}$.

\begin{tabular}{|c|c|c|c|c|l|}
\hline $\boldsymbol{k}_{\mathbf{3 1}}$ & $\boldsymbol{k}_{\mathbf{2 1}}$ & $\boldsymbol{I}_{\mathbf{1}} / \boldsymbol{I}_{\mathbf{3}}$ & $\boldsymbol{I}_{\mathbf{2}} / \boldsymbol{I}_{\mathbf{3}}$ & $\boldsymbol{C}_{\boldsymbol{L} \boldsymbol{\alpha}} / \boldsymbol{C}_{\boldsymbol{D}}$ & \multicolumn{1}{|c|}{ Resultado } \\
\hline 0 & $+20 \%$ & & & & Aumenta $t_{e e}$ \\
\hline 0 & $=0$ & & & & Disminuye $t_{e e}$ \\
\hline 0 & & $+20 \%$ & & & Disminuye $t_{e e}$ \\
\hline 0 & & & $+20 \%$ & & Aumenta $t_{e e}$ \\
\hline 0 & & & & $+20 \%$ & Aumenta $t_{e e}$ \\
\hline$>0$ & & & & & Aumenta $t_{e e}$ \\
\hline
\end{tabular}




\begin{tabular}{|c|c|c|c|c|l|}
\hline $\boldsymbol{k}_{\mathbf{3 1}}$ & $\boldsymbol{k}_{\mathbf{2 1}}$ & $\boldsymbol{I}_{\mathbf{1}} / \boldsymbol{I}_{\mathbf{3}}$ & $\boldsymbol{I}_{\mathbf{2}} / \boldsymbol{I}_{\mathbf{3}}$ & $\boldsymbol{C}_{\boldsymbol{L} \boldsymbol{\alpha}} / \boldsymbol{C}_{\boldsymbol{D}}$ & \multicolumn{1}{|c|}{ Resultado } \\
\hline$>0$ & $+20 \%$ & & & & Disminuye $t_{e e}$ \\
\hline$>0$ & $=0$ & & & & Aumenta $t_{e e}$ \\
\hline$>0$ & & $+20 \%$ & & & Aumenta $t_{e e}$ \\
\hline$>0$ & & & $+20 \%$ & & Disminuye $t_{e e}$ \\
\hline$>0$ & & & & $+20 \%$ & Aumenta $t_{e e}$ \\
\hline$<0$ & & & & & Aumenta $t_{e e}$ \\
\hline$<0$ & $+20 \%$ & & & & Disminuye $t_{e e}$ \\
\hline$<0$ & $=0$ & & & & Aumenta $t_{e e}$ \\
\hline$<0$ & & $+20 \%$ & & & Aumenta $t_{e e}$ \\
\hline$<0$ & & & $+20 \%$ & & Disminuye $t_{e e}$ \\
\hline$<0$ & & & & $+20 \%$ & Aumenta $t_{e e}$ \\
\hline
\end{tabular}

\subsection{CONCLUSIONES}

Las simulaciones numéricas muestran la existencia de tres tipos de soluciones. Un tipo de solución es la denominada Tipo I donde el ángulo de nutación de equilibrio es 0 , o muy cercano a 0 (si $\delta_{\beta} \neq 0$ ), que es la solución estable que resulta del modelo analítico. Otro tipo de solución es la denominada Tipo II, en la que el ángulo de nutación de equilibrio es mucho mayor que 0 grados, que se aleja de lo predicho por el modelo analítico (la Tipo I) implicando una actitud de vuelo del pararrotor con el eje 3 permanentemente inclinado respecto al eje inercial Z. El punto de bifurcación que da paso a esta solución depende de la relación de momentos de inercia, del valor $k_{31}$, del de $\delta_{\beta}$, y de la relación $C_{L \alpha} / C_{D}$ principalmente. Cuando el pararrotor se inclina más de 1,57 radianes (90 grados) aparece otra solución que se denomina funcionalmente inestable, o de inestabilidad funcional, por cuanto no mantiene la posición relativa del centro de masas con el plano que contiene a las palas, lo cual no es 
deseable desde el punto de vista funcional. En este caso la posición del centro de masas sobre eje 3 cambia mediante la rotación mencionada (se invierte) y evoluciona a la solución Tipo II con $k_{31}$ de signo contrario. Finalmente existe la posibilidad que la solución sea dinámicamente inestable.

De los casos simulados se observa que para determinados valores de coeficientes aerodinámicos de las palas, el modelo analítico y la simulación numérica son congruentes en la estimación de la solución de equilibrio cuando esta es de la denominada Tipo I. Los resultados de las simulaciones y del modelo analítico son congruentes y el error entre ellos es de pequeña magnitud.

Un aumento en el valor absoluto de $k_{31}$ al respecto de un caso de referencia hace que la bifurcación asociada con soluciones Tipo II aparezca a menores valores de $\delta_{\beta}$ que en el caso de referencia.

Las soluciones de equilibrio encontradas en las simulaciones introducen la existencia de soluciones con ángulo de nutación mucho mayor a 0 , no previstas el modelo analítico.

Estas simulaciones convalidan la existencia de regiones en el gráfico $N_{e}{ }^{\prime}, k_{e}$ que permiten predecir el comportamiento dinámico y la estabilidad del sistema.

Las simulaciones numéricas muestran el comportamiento del pararrotor incluyendo efectos no lineales y efectos de órdenes de magnitud menores no contemplados en el modelo analítico. En particular se hallan tres tipos de regiones coincidentes con las soluciones que se pueden encontrar, que incluye las soluciones Tipo I, Tipo II e inestables.

Se puede observar que la relación entre momentos de inercia principales son definitorios para la dinámica del sistema. Por un lado, como en el modelo analítico, es posible clasificar su comportamiento según los denominados Caso A $\left(I_{3}>I_{2}, I_{1}\right)$, Caso B $\left(I_{2}>I_{3}>I_{1}\right)$, Caso C $\left(I_{1}>\right.$ $\left.I_{3}>I_{2}\right)$, y Caso $\mathrm{D}\left(I_{3}<I_{2}, I_{1}\right)$. Por otro lado la relación $I_{2} / I_{3}, I_{1} / I_{3}$, condiciona la respuesta dinámica de los sistemas dados por estos casos. 
Si consideramos el Caso A, un alejamiento del valor de $I_{3}$ al respecto de $I_{2}, I_{1}$, mejora la estabilidad del sistema, y a la inversa, su aproximación tiene como límite a la inestabilidad dinámica, independientemente del valor que tome $k_{31}$, como se observa en las simulaciones realizadas mencionadas anteriormente.

Si tomamos en consideración el caso base de configuración de momentos de inercia es posible apreciar que si partimos de una solución estable para $k_{31}=0$, aumentos de signo positivo del parámetro $k_{31}$ pueden causar cambios en el tipo de solución (de Tipo I a Tipo II) con distintos ángulos de nutación de equilibrio y de tiempos hasta alcanzar el equilibrio, pero no soluciones de inestabilidad dinámica. Incluso es posible alcanzar soluciones estables que resultan inestables para $k_{31}=0 . \mathrm{Si}$ este aumento es de signo negativo es posible alcanzar soluciones Tipo I, Tipo II o inestabilidad dinámica, en función de las magnitudes de los parámetros característicos del pararrotor. Incluso valores negativos de $k_{31}$ pueden inestabilizar sistemas que para $k_{31}=0$ muestran una solución estable.

Al respecto de parámetros de desempeño, los ensayos realizados muestran que para $I_{3}>I_{2}, I_{1}$, el parámetro $k$ crece linealmente con $\beta_{0}$, mientras que el parámetro $c_{\mathrm{DM}}$ tiene un valor mínimo para determinado $\beta_{0}$ con una función polinomial que describe su comportamiento.

El Caso B muestra inestabilidad dinámica para $k_{31}=0$, que resulta de la configuración de los momentos de inercia. Resulta posible encontrar soluciones estables con $k_{31}>0$ que no resultan factibles tecnológicamente hablando.

El Caso C muestra, idénticamente al Caso $\mathrm{B}$, inestabilidad dinámica para $k_{31}=0$, que resulta de la configuración de los momentos de inercia. También en este caso es posible encontrar soluciones estables con $k_{31}=0$.

Si consideramos el Caso D, podemos ver que el efecto de los parámetros $I_{2} / I_{3}$ e $I_{1} / I_{3}$, se invierten al respecto del Caso A.

Dada una solución estable para el Caso D con $k_{31}=0$, la introducción de valores positivos de $k_{31}$ puede conllevar, igual que para el Caso A, cambio de una solución Tipo I a Tipo II, cambio del ángulo de nutación y del tiempo hasta alcanzar la solución de equilibrio, pero no 
inestabilidad dinámica o funcional. Valores positivos de $k_{31}$ pueden incluso estabilizar soluciones que son inestables para $k_{31}=0$. Si se adoptan valores de $k_{31}$ negativos, entonces es posible además alcanzar inestabilidad funcional. Valores negativos de $k_{31}$ pueden incluso inestabilizar soluciones que son estables para $k_{31}=0$.

Si analizamos el efecto del parámetro $k_{21}$, para el Caso A es posible observar que para $k_{31}=0$ su efecto es el de disminuir el tiempo hasta alcanzar la solución de equilibrio mientras más grande sea su magnitud. Cuando el caso es que $k_{31}>0$ un aumento de $k_{21}$ conlleva un aumento de tiempo, y si $k_{31}<0$ una disminución.

Si analizamos el efecto del parámetro $k_{21}$ para el Caso D es posible observar que para $k_{31}=0$ su efecto es el de aumentar el tiempo hasta alcanzar la solución de equilibrio mientras más grande sea su magnitud. Cuando el caso es que $k_{31}>0$ y $k_{31}<0$ un aumento de $k_{21}$ conlleva una disminución de tiempo.

Si analizamos el efecto de $C_{L \alpha} / C_{D}$ para el Caso A y en Caso D, se puede observar en los casos simulados que cuando $k_{31}=0, k_{31}>0$ y $k_{31}<0$ el efecto de su incremento es el de aumentar el tiempo hasta alcanzar la solución de equilibrio, a la vez que acerca al sistema a los límites de estabilidad.

\subsection{CONCLUSIONES GENERALES}

La conclusión general del presente capítulo es que a través de la variación de $k_{31}, C_{L \alpha}, C_{D}, \beta_{1}$ y $\beta_{2}$ es posible controlar el comportamiento dinámico de un pararrotor dado (es decir, fijados los momentos de inercia, los parámetros mencionados pueden modificarse en vuelo para cambiar la respuesta dinámica del dispositivo). En particular el tiempo de respuesta del sistema a una perturbación y la solución de equilibrio que determina la actitud del pararrotor son dependientes de estos parámetros. Es posible por tanto controlar y guiar este tipo de dispositivos por medio de la manipulación de los parámetros mencionados. 
En los estudios analíticos se presentan dos parámetros asociados a la estabilidad de los sistemas, $N_{e}{ }^{\prime}$ y $k_{e}$, que resultan de la linealización de las ecuaciones eliminando aquellas magnitudes de menor orden en su expresión analítica. Se comprueba en las simulaciones numéricas realizadas que estos dos parámetros son útiles para describir las condiciones de estabilidad del sistema al agrupar el elevado número de parámetros que intervienen en solo dos.

En los estudios analíticos se establecieron distintos casos de estabilidad en función de las relaciones de momentos de inercia. Estas relaciones han sido útiles para la descripción de la estabilidad del sistema en las simulaciones realizadas.

En general se demuestra a partir de las simulaciones realizadas que $k_{31}$ contribuye más a la estabilidad del sistema cuanto mayor sea (a pesar de que su introducción promueva la aparición de soluciones de equilibrio tipo cónicas). 


\section{CAPÍTULO 4}

\section{ESTUDIO EXPERIMENTAL DEL EFECTO DEL DESPLAZAMIENTO DEL PLANO QUE CONTIENE A LAS PALAS CON RESPECTO AL CENTRO DE MASAS EN LA DINÁMICA DEL VUELO DE UN DESACELERADOR AERODINÁMICO DE ALAS ROTATORIAS}

\subsection{INTRODUCCIÓN}

Para el estudio experimental del dispositivo desacelerador basado en la autorrotación de alas giratorias, pararrotor, se prepararon una serie de ensayos sobre la base de modelos físicos del dispositivo.

En el presente apartado se exponen las investigaciones experimentales realizadas en el tema, a saber:

a) Ensayos en túnel aerodinámico vertical sobre un modelo en vuelo libre.

b) Ensayos en túnel aerodinámico sobre un modelo que rota sobre un rodamiento basculante.

El objeto del presente apartado es caracterizar el comportamiento dinámico del pararrotor a través de ensayos experimentales, útiles para disponer de elementos para validar los modelos teóricos y el simulador numérico. 


\subsection{ENSAYOS EN TÚNEL AERODINÁMICO VERTICAL SOBRE UN MODELO EN VUELO LIBRE}

\subsubsection{Descripción del modelo físico y configuración experimental}

La configuración experimental se basa en un túnel aerodinámico vertical, donde se realizan los ensayos de la presente serie de modelos físicos, que se muestra esquemáticamente en la Figura 4.1. El túnel vertical consiste en un soplador que impulsa un caudal de aire sobre una sección de ensayo donde el modelo físico de pararrotor realiza un vuelo libre. El túnel está compuesto por una entrada de aire asociada a un ventilador axial de $5500 \mathrm{~m}^{3} / \mathrm{h}$ situado en la parte inferior del túnel. El aire impulsado descarga a un difusor, a través de un enderezador del flujo. La sección de prueba se encuentra dentro del mencionado difusor. La forma del difusor fue diseñada para obtener una velocidad de descenso vertical constante del modelo en condiciones de vuelo estable, de forma que el modelo físico de pararrotor mantenga el nivel vertical. El difusor tiene las siguientes características geométricas:

- sección rectangular de profundidad constante de 0,28 m, ancho mínimo de 0,24 m, ancho máximo de $0,57 \mathrm{~m}$ y largo de $2,25 \mathrm{~m}$,

- sección de prueba rectangular de ancho mínimo de 0,4 m, ancho máximo de 0,46 m y largo de $0,5 \mathrm{~m}$.

Centrado en el interior de la sección de prueba se dispone de un cable guía que limita el desplazamiento lateral (sobre los ejes inerciales $X$ e $Y$ ) del modelo.

Los modelos físicos ensayados consisten en un cilindro hueco y dos palas planas rectangulares de aluminio. El modelo tipo se describe esquemáticamente en la Figura 4.2. Situada en el centro de masas se encuentra una perforación en una placa que permite al modelo deslizarse a lo largo del cable guía de manera que sólo se limita parcialmente el desplazamiento lateral. Para el presente análisis se utilizaron dos juegos de cilindros y tres juegos de palas. Las combinaciones de juegos de palas y cilindros dan diferentes relaciones entre los valores de los momentos de inercia principales del modelo completo. 


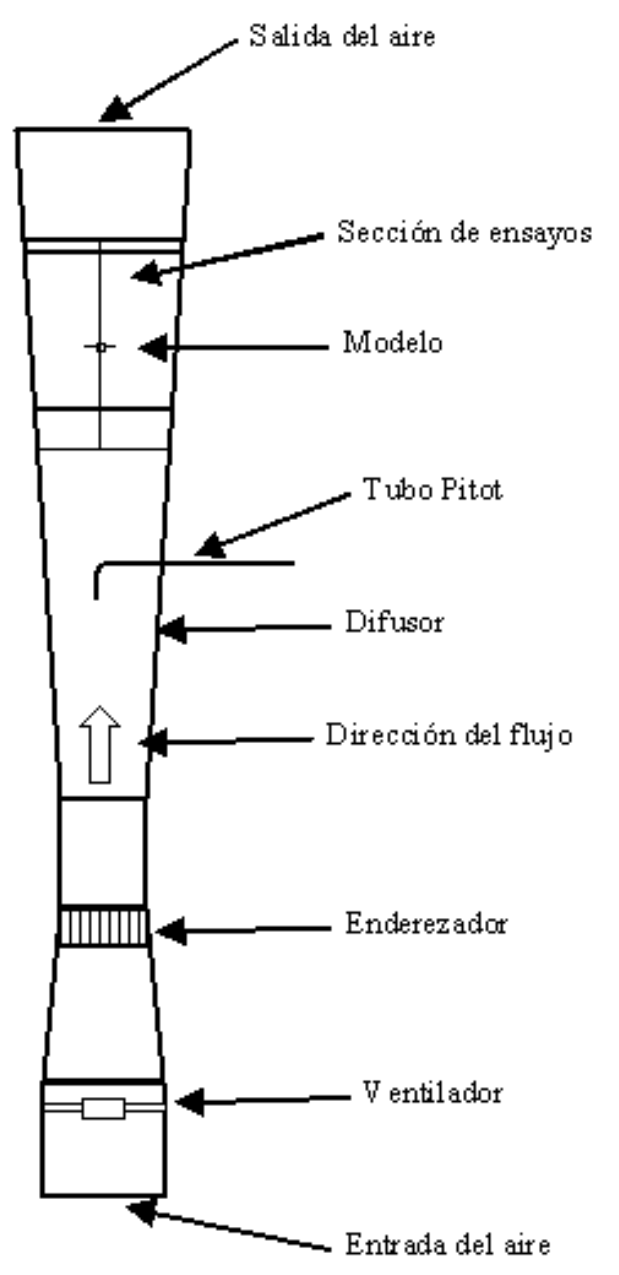

Figura 0.1 Esquema de la instalación experimental del túnel vertical 

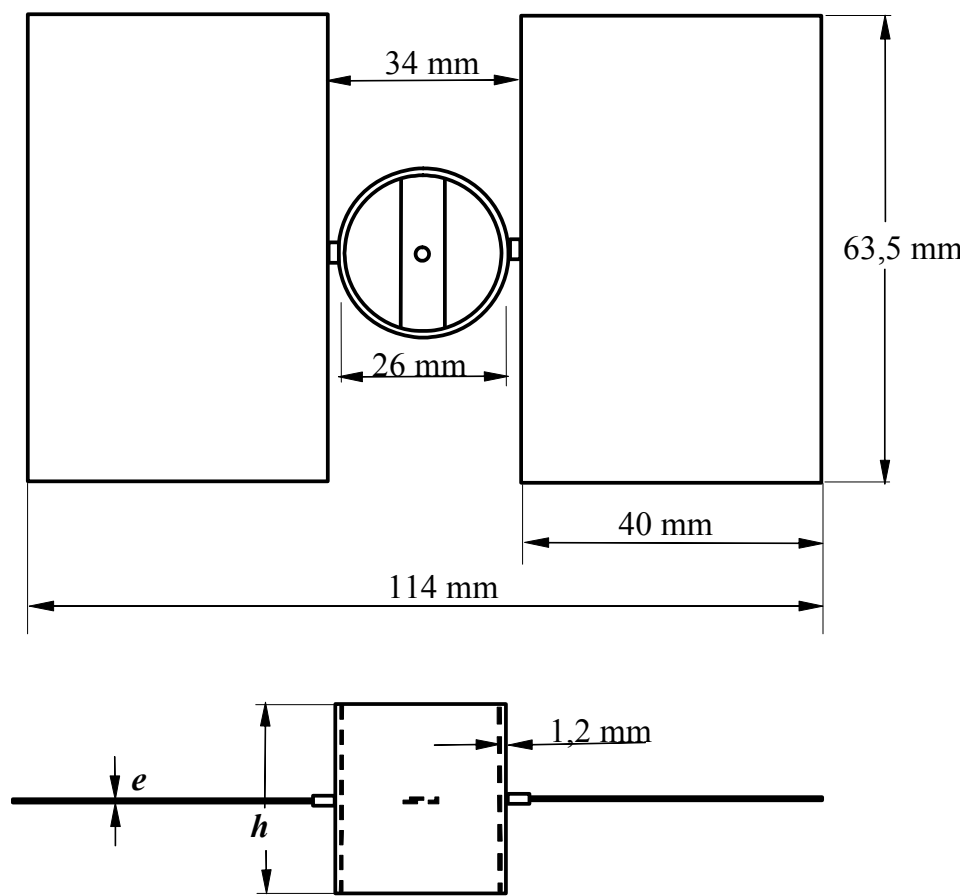

Figura 0.2 Esquema y dimensiones del modelo físico del pararrotor

Las dimensiones y parámetros geométricos de los modelos ensayados se muestran en las Tabla 4.1.

Tabla 0.1 Parámetros distintivos de los modelos del pararrotor ensayados.

\begin{tabular}{|c|c|c|c|c|c|c|}
\hline Denominación & $\begin{array}{c}\text { Peso } \\
{[\mathbf{N}]}\end{array}$ & $\begin{array}{c}h \\
{[\mathrm{~mm}]}\end{array}$ & $\begin{array}{c}e \\
{[\mathrm{~mm}]}\end{array}$ & $\begin{array}{c}I_{1} \\
{\left[\mathrm{~kg} \mathrm{~m}^{2}\right]}\end{array}$ & $\begin{array}{c}I_{2} \\
{\left[\mathrm{~kg} \mathrm{~m}^{2}\right]}\end{array}$ & $\begin{array}{c}I_{3} \\
{\left[\mathrm{~kg} \mathrm{~m}^{2}\right]}\end{array}$ \\
\hline C05P01 & 0,15 & 26 & 0,5 & $3,5 \cdot 10^{-6}$ & $8,9 \cdot 10^{-6}$ & $11,6 \cdot 10^{-6}$ \\
\hline C05P02 & 0,20 & 26 & 0,8 & $5,4 \cdot 10^{-6}$ & $21,2 \cdot 10^{-6}$ & $25,9 \cdot 10^{-6}$ \\
\hline$C 05 P 03$ & 0,11 & 26 & 0,3 & $1,9 \cdot 10^{-6}$ & $5,8 \cdot 10^{-6}$ & $6,9 \cdot 10^{-6}$ \\
\hline
\end{tabular}




\begin{tabular}{|c|c|c|c|c|c|c|}
\hline Denominación & $\begin{array}{c}\text { Peso } \\
{[\mathbf{N}]}\end{array}$ & $\begin{array}{c}\boldsymbol{h} \\
{[\mathbf{m m}]}\end{array}$ & $\begin{array}{c}\boldsymbol{e} \\
{[\mathbf{m m}]}\end{array}$ & $\begin{array}{c}\boldsymbol{I}_{\mathbf{1}} \\
{\left[\mathbf{k g ~ \mathbf { m } ^ { 2 } ]}\right.}\end{array}$ & $\begin{array}{c}\boldsymbol{I}_{\mathbf{2}} \\
{\left[\mathbf{k g ~ m}^{2}\right]}\end{array}$ & $\begin{array}{c}\mathbf{I}_{3} \\
{\left[\mathbf{k g ~ \mathbf { m } ^ { 2 } ]}\right.}\end{array}$ \\
\hline$C 06 P 02$ & 0,28 & 56 & 0,8 & $9,5 \cdot 10^{-6}$ & $25,3 \cdot 10^{-6}$ & $27,1 \cdot 10^{-6}$ \\
\hline$C 06 P 03$ & 0,19 & 56 & 0,3 & $5,9 \cdot 10^{-6}$ & $9,8 \cdot 10^{-6}$ & $8,1 \cdot 10^{-6}$ \\
\hline$C 05 P 02 M 01$ & $0,22^{*}$ & 26 & 0,8 & $10,1 \cdot 10^{-6}$ & $22,5 \cdot 10^{-6}$ & $31,8 \cdot 10^{-6}$ \\
\hline
\end{tabular}

*se incluyen dos masas de 2,2 gr situadas en las coordenadas ejes cuerpo: (17 mm, $-32 \mathrm{~mm}, 0 \mathrm{~mm})$ y $(-17 \mathrm{~mm}$, $32 \mathrm{~mm}, 0 \mathrm{~mm})$.

\subsubsection{Parámetros medidos e instrumentación}

Los parámetros que fueron medidos para estos ensayos son:

- velocidad de rotación, por medio de una lámpara estroboscópica,

- paso de las palas, por medio de un goniómetro,

- velocidad de la corriente, por medio de un tubo Pitot estandarizado y un micromanómetro,

- peso del modelo, por medio de una balanza electrónica.

- ángulo de nutación promedio, por medio de fotografía convencional sobre un registro de fondo

- temperatura ambiente, mediante un termómetro de bulbo húmedo y bulbo seco

- presión barométrica, mediante un barómetro electrónico

El instrumental utilizado se detalla en la Tabla 4.2.

Tabla 0.2 Descripción del instrumental utilizado en los ensayos de túnel vertical.

\begin{tabular}{|c|c|c|c|c|}
\hline Instrumento & Marca & Modelo/Tipo & Rango & Resolución \\
\hline
\end{tabular}




\begin{tabular}{|c|c|c|c|c|}
\hline Instrumento & Marca & Modelo/Tipo & Rango & Resolución \\
\hline $\begin{array}{c}\text { Lámpara } \\
\text { estroboscópica }\end{array}$ & - & - & - & $50 \mathrm{rpm}$ \\
\hline Tacómetro Láser & Lutron & DT-2234BL & 10 a $99999 \mathrm{rpm}$ & $\begin{array}{c}0,1 \mathrm{rpm}(<1000 \\
\mathrm{rpm}) \\
1 \mathrm{rpm}(>1000 \mathrm{rpm}) \\
\end{array}$ \\
\hline Tubo Pitot & Estándar & $\begin{array}{c}\text { NPL } \\
\text { Iram } 19004\end{array}$ & - & - \\
\hline $\begin{array}{c}\text { Micromanómetro } \\
\text { diferencial }\end{array}$ & R-Fuess & & 0 a $160 \mathrm{mmCA}$ & $0,05 \mathrm{mmCA}$ \\
\hline Barómetro & Druck & DPI740 & 0 a $400 \mathrm{~Pa}$ & $0,1 \mathrm{~Pa}$ \\
\hline $\begin{array}{c}\text { Termómetro de } \\
\text { bulbo seco y } \\
\text { bulbo húmedo }\end{array}$ & & & $\begin{array}{c}\mathrm{BH}-25^{\circ} \mathrm{C} \mathrm{a} \\
35^{\circ} \mathrm{C} \\
\mathrm{BS} 0^{\circ} \mathrm{C} \text { a } 100^{\circ} \mathrm{C} \\
\end{array}$ & $0,1^{\circ} \mathrm{C}$ \\
\hline $\begin{array}{l}\text { Balanza } \\
\text { electrónica }\end{array}$ & - & - & $\begin{array}{l}0 \text { a } 5000 \\
\text { gramos }\end{array}$ & 10 gramos \\
\hline Goniómetro & - & - & 0 a 90 grados & 1 grado \\
\hline $\begin{array}{l}\text { Cámara } \\
\text { fotográfica sobre } \\
\text { registro de fondo }\end{array}$ & Sony & DSC-W110 & - & - \\
\hline
\end{tabular}

\subsubsection{Definiciones}

Se determina el coeficiente de resistencia del modelo, $c_{D M}$, tomando como área de referencia a la superficie de las dos palas: $S_{P}=2 b c$. El coeficiente de resistencia del modelo se define como:

$$
c_{D M}=\frac{T}{\frac{1}{2} \rho V_{v}^{2} S_{p}}
$$


Para calcular el número de Reynolds del fenómeno ensayado se utilizó como longitud característica la cuerda de las palas. Adicionalmente se define:

$V_{t}=\omega_{3} R_{60 \%}$,

donde $\mathrm{R}_{60 \%}$ es el radio al $60 \%$ de la envergadura de la pala. El Reynolds se define entonces como:

$$
\operatorname{Re}_{P}=\frac{V_{t} c}{v}
$$

Se define $k$ a la relación de velocidades:

$$
k=\frac{V_{v}}{\omega_{3} R},
$$

donde $R=0,057 \mathrm{~m}$.

Adicionalmente se define el ángulo del plano medio entre las palas:

$$
\delta_{\beta}=\frac{\beta_{1}-\beta_{2}}{2}
$$

y el ángulo relativo entre las palas:

$$
\varepsilon=\beta_{1}+\beta_{2}
$$

\subsubsection{Ensayos realizados y resultados}

Se han realizado diferentes ensayos sobre los modelos descriptos en la Tabla 4.1 para los ángulos de paso de las palas mostrados en las Tablas 4.3, Tabla 4.4 y Tabla 4.5, donde también se presentan los valores medios de los parámetros $k \mathrm{y} c_{D M}$.

Tabla 0.3 Ensayos realizados en el túnel vertical y resultados promedio de $k \mathrm{y} c_{D M}$. 


\begin{tabular}{|c|c|c|c|c|c|c|c|}
\hline Configuración & $\operatorname{Re}$ & $\begin{array}{l}\beta_{1} \\
{\left[^{0}\right]}\end{array}$ & $\begin{array}{l}\beta_{2} \\
{\left[^{0}\right]}\end{array}$ & $\begin{array}{c}\delta_{\beta} \\
{\left[^{\circ}\right]}\end{array}$ & $\begin{array}{c}\boldsymbol{\varepsilon} \\
{\left[^{0}\right]}\end{array}$ & $\boldsymbol{k}$ & $c_{D M}$ \\
\hline$C 05 P 01$ & 40.960 & -12 & 10 & -11 & -2 & 0,44 & 3,32 \\
\hline$C 05 P 02$ & 45.488 & -12 & 10 & -11 & -2 & 0,46 & 3,38 \\
\hline$C 05 P 02$ & 53.672 & -12 & 8 & -10 & -4 & 0,39 & 3,06 \\
\hline$C 05 P 02$ & 47.624 & -10 & 8 & -9 & -2 & - & - \\
\hline C05P02 & 55.302 & -10 & 6 & -8 & -4 & 0,36 & 3,25 \\
\hline$C 05 P 02$ & 60.198 & -10 & 4 & -7 & -6 & 0,32 & 3,3 \\
\hline C05P02 & 45.488 & -10 & -10 & 0 & -20 & 0,46 & 3,38 \\
\hline С05P02 & 44.935 & -8 & 10 & -9 & 2 & 0,49 & 3,19 \\
\hline$C 05 P 02$ & 48.510 & -8 & 6 & -7 & -2 & 0,4 & 3,61 \\
\hline C05P02 & 52.342 & -8 & 4 & -6 & -4 & 0,37 & 3,48 \\
\hline$C 05 P 02$ & 56.434 & -8 & 2 & -5 & -6 & 0,34 & 3,41 \\
\hline$C 05 P 02$ & 75.729 & -8 & 0 & -4 & -8 & 0,24 & 3,39 \\
\hline С05P02 & 83.689 & -8 & -4 & -2 & -12 & 0,22 & 3,24 \\
\hline$C 05 P 02$ & 90.847 & -8 & -8 & 0 & -16 & 0,21 & 2,99 \\
\hline$C 05 P 02$ & 74.929 & -8 & -8 & 0 & -16 & 0,32 & 2,13 \\
\hline$C 05 P 02$ & 46.027 & -6 & 4 & -5 & -2 & 0,45 & 3,4 \\
\hline$C 05 P 02$ & 52.214 & -6 & 2 & -4 & -4 & 0,38 & 3,36 \\
\hline$C 05 P 02$ & 78.190 & -6 & -6 & 0 & -12 & 0,24 & 3,18 \\
\hline$C 05 P 02$ & 86.663 & -6 & -8 & 1 & -14 & 0,25 & 2,41 \\
\hline$C 05 P 02$ & 72.246 & -4 & -4 & 0 & -8 & 0,26 & 3,24 \\
\hline
\end{tabular}




\begin{tabular}{|c|c|c|c|c|c|c|c|}
\hline Configuración & $\operatorname{Re}$ & $\begin{array}{c}\beta_{1} \\
{\left[^{\circ}\right]}\end{array}$ & $\begin{array}{l}\beta_{2} \\
{\left[^{0}\right]}\end{array}$ & $\begin{array}{c}\delta_{\beta} \\
{\left[^{0}\right]}\end{array}$ & $\begin{array}{c}\boldsymbol{\varepsilon} \\
{\left[^{\circ}\right]}\end{array}$ & $\boldsymbol{k}$ & $c_{D M}$ \\
\hline C05P02 & 74.956 & -4 & -6 & 1 & -10 & 0,26 & 3,01 \\
\hline$C 05 P 02$ & 79.693 & -4 & -8 & 2 & -12 & 0,25 & 2,85 \\
\hline C05P02 & 67.883 & -2 & -2 & 0 & -4 & 0,27 & 3,44 \\
\hline C05P02M01 & 89.064 & -8 & -8 & 0 & -16 & 0,25 & 2,51 \\
\hline C05P02M01 & 81.580 & -6 & -6 & 0 & -12 & 0,27 & 2,62 \\
\hline C05P02M01 & 70.201 & -4 & -4 & 0 & -8 & 0,31 & 2,81 \\
\hline C05P02M01 & 57.668 & -2 & -2 & 0 & -4 & 0,38 & 3,03 \\
\hline C05P03 & 51.450 & -12 & 10 & -11 & -2 & 0,39 & 3,33 \\
\hline C05P03 & 53.366 & -10 & 8 & -9 & -2 & 0,36 & 3,49 \\
\hline$C 05 P 03$ & 60.163 & -6 & 0 & -3 & -6 & 0,3 & 3,67 \\
\hline$C 05 P 03$ & 79.708 & -6 & -6 & 0 & -12 & 0,24 & 3,06 \\
\hline C05P03 & 62.691 & -4 & -4 & 0 & -8 & 0,3 & 3,38 \\
\hline$C 05 P 03$ & 73.726 & -4 & -6 & 1 & -10 & 0,25 & 3,33 \\
\hline C06P02 & 88.657 & -8 & -8 & 0 & -16 & 0,32 & 2,13 \\
\hline С06P02 & 108.783 & -6 & -6 & 0 & -12 & 0,24 & 2,3 \\
\hline С06P02 & 98.229 & -4 & -4 & 0 & -8 & 0,27 & 2,3 \\
\hline С06Р02 & 83.300 & -2 & -2 & 0 & -4 & 0,31 & 2,54 \\
\hline С06P03 & 89.611 & -6 & -6 & 0 & -12 & 0,24 & Inestable \\
\hline С06P03 & 80.916 & -4 & -4 & 0 & -8 & 0,27 & Inestable \\
\hline С06Р03 & 68.619 & -2 & -2 & 0 & -4 & 0,31 & Inestable \\
\hline
\end{tabular}


Tabla 0.4 Ensayos realizados en el túnel vertical y valores calculados de $N_{e}$ ' y $k_{e}$.

\begin{tabular}{|c|c|c|c|c|c|c|}
\hline Configuración & $\begin{array}{l}\beta_{1} \\
{\left[^{0}\right]}\end{array}$ & $\begin{array}{c}\beta_{2} \\
{\left[^{\circ}\right]}\end{array}$ & $\begin{array}{c}\delta_{\beta} \\
{\left[^{\circ}\right]}\end{array}$ & $\begin{array}{c}\varepsilon \\
{\left[^{\circ}\right]}\end{array}$ & $N_{e}^{\prime}$ & $\boldsymbol{k}_{e}$ \\
\hline$C 05 P 01$ & -12 & 10 & -11 & -2 & 0,03197 & 2,169 \\
\hline C05P02 & -12 & 10 & -11 & -2 & 0,01866 & 3,181 \\
\hline$C 05 P 02$ & -12 & 8 & -10 & -4 & 0,01866 & 3,181 \\
\hline C05P02 & -10 & 8 & -9 & -2 & 0,01866 & 3,181 \\
\hline C05P02 & -10 & 6 & -8 & -4 & 0,01866 & 3,181 \\
\hline$C 05 P 02$ & -10 & 4 & -7 & -6 & 0,01866 & 3,181 \\
\hline$C 05 P 02$ & -10 & -10 & 0 & -20 & 0,01866 & 3,181 \\
\hline$C 05 P 02$ & -8 & 10 & -9 & 2 & 0,01866 & 3,181 \\
\hline$C 05 P 02$ & -8 & 6 & -7 & -2 & 0,01866 & 3,181 \\
\hline$C 05 P 02$ & -8 & 4 & -6 & -4 & 0,01866 & 3,181 \\
\hline C05P02 & -8 & 2 & -5 & -6 & 0,01866 & 3,181 \\
\hline$C 05 P 02$ & -8 & 0 & -4 & -8 & 0,01866 & 3,181 \\
\hline$C 05 P 02$ & -8 & -4 & -2 & -12 & 0,01866 & 3,181 \\
\hline$C 05 P 02$ & -8 & -8 & 0 & -16 & 0,01866 & 3,181 \\
\hline$C 05 P 02$ & -8 & -8 & 0 & -16 & 0,0187 & 3,181 \\
\hline$C 05 P 02$ & -6 & 4 & -5 & -2 & 0,0187 & 3,181 \\
\hline$C 05 P 02$ & -6 & 2 & -4 & -4 & 0,0187 & 3,181 \\
\hline$C 05 P 02$ & -6 & -6 & 0 & -12 & 0,0187 & 3,181 \\
\hline$C 05 P 02$ & -6 & -8 & 1 & -14 & 0,0187 & 3,181 \\
\hline$C 05 P 02$ & -4 & -4 & 0 & -8 & 0,0187 & 3,181 \\
\hline$C 05 P 02$ & -4 & -6 & 1 & -10 & 0,0187 & 3,181 \\
\hline$C 05 P 02$ & -4 & -8 & 2 & -12 & 0,0187 & 3,181 \\
\hline$C 05 P 02$ & -2 & -2 & 0 & -4 & 0,0187 & 3,181 \\
\hline C05P02M01 & -8 & -8 & 0 & -16 & 0,6280 & 10,030 \\
\hline C05P02M01 & -6 & -6 & 0 & -12 & 0,6284 & 10,030 \\
\hline
\end{tabular}




\begin{tabular}{|c|c|c|c|c|c|c|}
\hline Configuración & $\begin{array}{c}\beta_{1} \\
{\left[^{\circ}\right]}\end{array}$ & $\begin{array}{c}\beta_{2} \\
{\left[^{0}\right]}\end{array}$ & $\begin{array}{c}\boldsymbol{\delta}_{\beta} \\
{\left[^{\circ}\right]}\end{array}$ & $\begin{array}{c}\boldsymbol{\varepsilon} \\
{\left[^{\circ}\right]}\end{array}$ & $\boldsymbol{N}_{\boldsymbol{e}}{ }^{\prime}$ & $\boldsymbol{k}_{\boldsymbol{e}}$ \\
\hline$C 05 P 02 M 01$ & -4 & -4 & 0 & -8 & 0,6284 & 10,030 \\
$C 05 P 02 M 01$ & -2 & -2 & 0 & -4 & 0,6284 & 10,030 \\
$C 05 P 03$ & -12 & 10 & -11 & -2 & 0,0187 & 3,181 \\
$C 05 P 03$ & -10 & 8 & -9 & -2 & 0,0187 & 3,181 \\
$C 05 P 03$ & -6 & 0 & -3 & -6 & 0,0187 & 3,181 \\
$C 05 P 03$ & -6 & -6 & 0 & -12 & 0,0187 & 3,181 \\
$C 05 P 03$ & -4 & -4 & 0 & -8 & 0,0187 & 3,181 \\
$C 05 P 03$ & -4 & -6 & 1 & -10 & 0,0187 & 3,181 \\
$C 06 P 02$ & -8 & -8 & 0 & -16 & 0,0471 & 6,912 \\
$C 06 P 02$ & -6 & -6 & 0 & -12 & 0,0471 & 6,912 \\
$C 06 P 02$ & -4 & -4 & 0 & -8 & 0,0471 & 6,912 \\
$C 06 P 02$ & -2 & -2 & 0 & -4 & 0,0471 & 6,912 \\
$C 06 P 03$ & -6 & -6 & 0 & -12 & $-0,0528$ & $-0,9726$ \\
$C 06 P 03$ & -4 & -4 & 0 & -8 & $-0,0528$ & $-0,9726$ \\
$C 06 P 03$ & -2 & -2 & 0 & -4 & $-0,0528$ & $-0,9726$ \\
\hline
\end{tabular}

Tabla 0.5 Ensayos realizados en el túnel vertical y valores calculados de $N_{e}{ }^{\prime}$ y $k_{e}$.

\begin{tabular}{|c|c|c|c|c|c|}
\hline Configuración & $\begin{array}{c}\beta_{1,}, \beta_{2} \\
\left.{ }^{0}{ }^{0}\right]\end{array}$ & $\begin{array}{c}\boldsymbol{V}_{\boldsymbol{v}} \\
{[\mathrm{m} / \mathrm{s}]}\end{array}$ & $\begin{array}{c}\omega_{3} \\
{[\mathrm{rad} / \mathrm{s}]}\end{array}$ & $N_{e}^{\prime}$ & $\boldsymbol{k}_{e}$ \\
\hline C05P02 & -8 & 4,92 & 312 & 0,0187 & 3,181 \\
\hline$C 05 P 02$ & -6 & 4,72 & 298 & 0,0187 & 3,181 \\
\hline С05P02 & -4 & 4,66 & 291 & 0,0187 & 3,181 \\
\hline$C 05 P 02$ & -2 & 4,58 & 287 & 0,0187 & 3,181 \\
\hline$C 05 P 03$ & -6 & 3,50 & 260 & 0,0187 & 3,181 \\
\hline$C 05 P 03$ & -4 & 3,32 & 197 & 0,0187 & 3,181 \\
\hline С06P02 & -6 & 5,50 & 398 & 0,0471 & 6,912 \\
\hline С06P02 & -4 & 5,50 & 356 & 0,0471 & 6,912 \\
\hline$C 06 P 02$ & -2 & 5,25 & 316 & 0,0471 & 6,912 \\
\hline
\end{tabular}




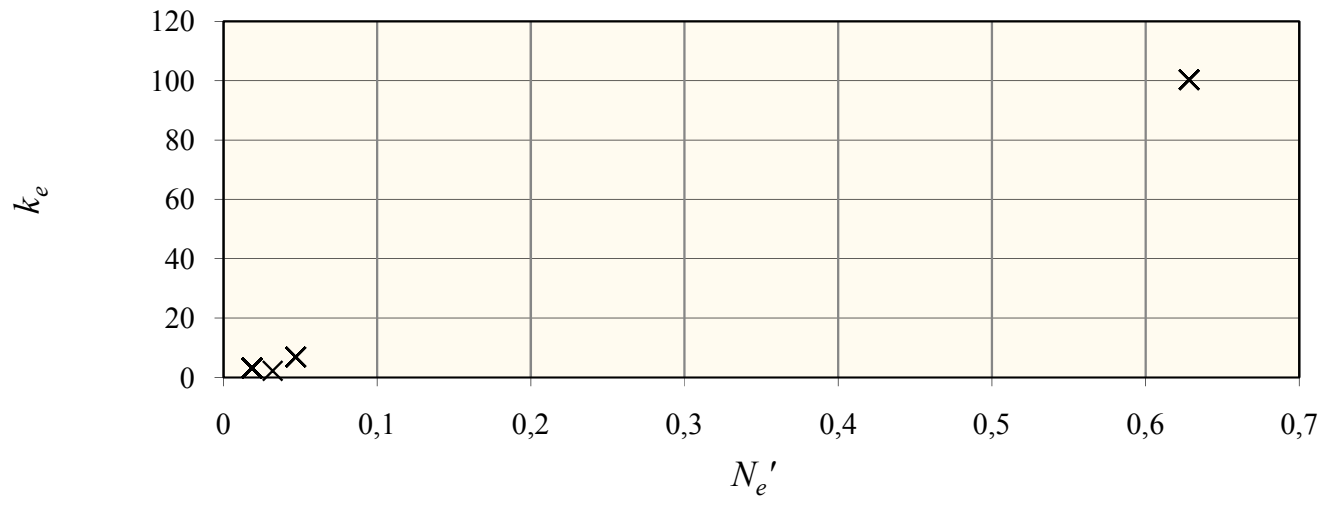

Figura 0.3 Diagrama $\left(N_{e}, k_{e}\right)$ de los casos ensayados que se presentan en la Tabla 4.3

La Figura 4.3 agrupa cualitativamente casos ensayados en función del ángulo de nutación de equilibrio del pararrotor sobre un diagrama $\left(N_{e}{ }^{\prime}, k_{e}\right)$. Los casos son correspondientes al caso donde $I_{3}>I_{2}, I_{1}$, salvo los ensayos realizados con la configuración C06P03. Se puede observar la existencia de dos tipos de soluciones cualitativamente distintas que permiten agrupar los ensayos realizados. Un tipo de solución (que se denomina Tipo I, tal cual se presenta en el Capítulo 3) es aquella donde el ángulo de nutación de equilibrio es cero, o muy cercano, y representa la generalidad de los casos ensayados. Esta solución de equilibrio se muestra en la Figura 4.4. Finalmente otra alternativa de evolución del sistema que se presenta es la de la inestabilidad dinámica, que se corresponden a los ensayos realizados sobre la configuración C06P03. Se muestra esta respuesta en la Figura 4.5. 


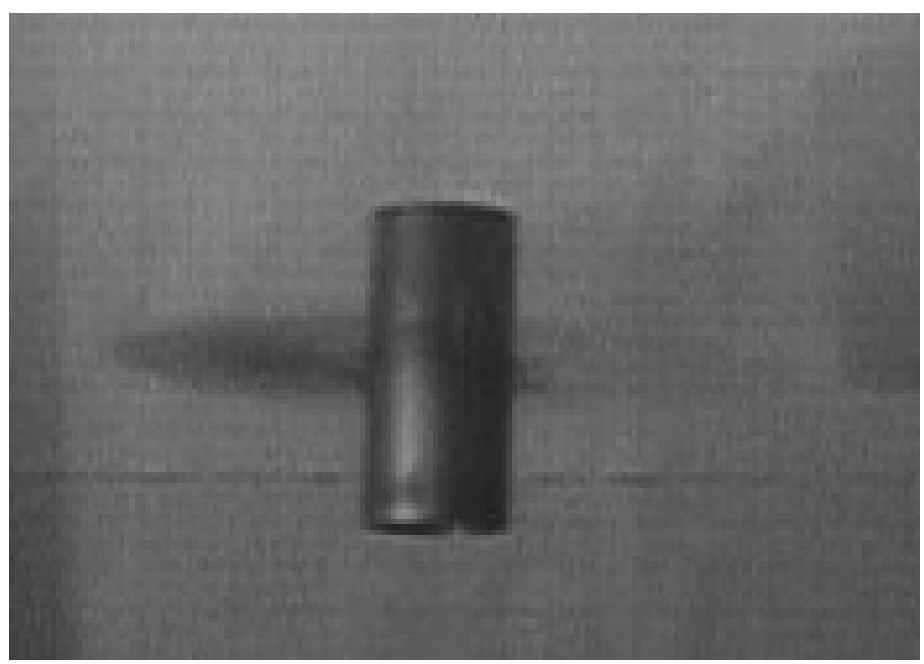

Figura 0.4 Actitud del modelo C06P02, para $\beta_{1}=\beta_{2}=-4^{\circ} . I_{3}>I_{1}, I_{2}$. Movimiento estable.

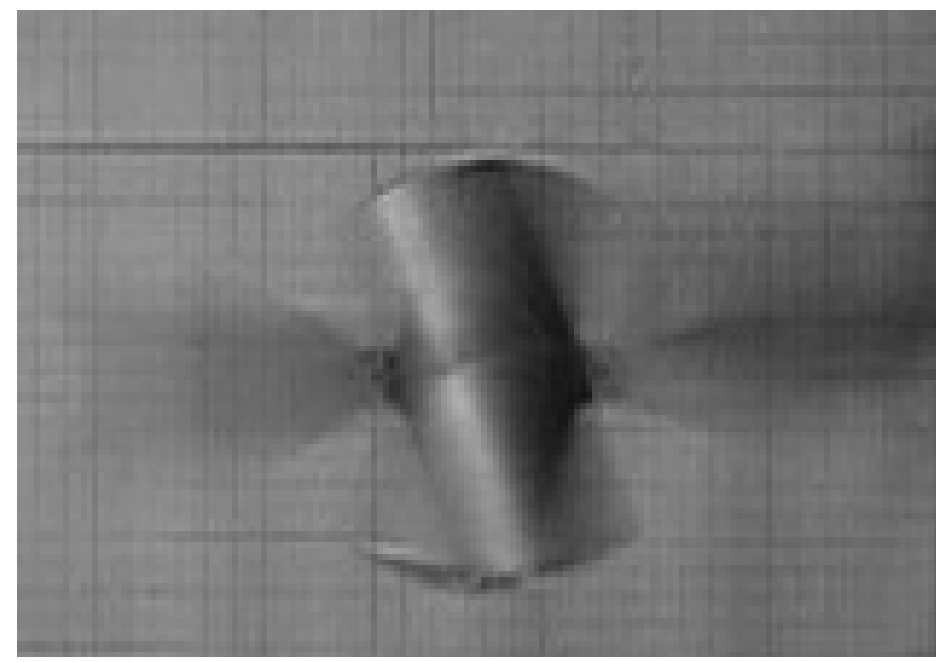

Figura 0.5 Actitud del modelo C06P03, para $\beta_{1}=\beta_{2}=-4^{\circ} . I_{2}>I_{3}>I_{1}$. Movimiento inestable.

\subsubsection{Análisis de los resultados}

Es posible observar que para las configuraciones con ángulos de paso de ambas palas iguales $(-2,-4,-6, y-8$ grados $)$, la relación de velocidades $k$ crecen al aumentar el ángulo de paso de 
las palas, como se muestra en las Figuras 4.6. La pendiente que se muestra en la recta de tendencia es de 0,010 1/grado.

Por otro lado los ensayos realizados muestran que el coeficiente de resistencia del modelo $c_{D M}$ disminuye al aumentar el paso de las palas, con una curva de tendencia cuadrática, como se muestra en las Figuras 4.7. Este hecho indica que los valores máximos de $c_{D M}$ se alcanzan a ángulos mínimos de $\beta_{1}$ y $\beta_{2}$.

Al respecto de la diferencia de paso de palas $\delta_{\beta}$, puede observarse que $k$ decrece al crecer $\delta_{\beta}$.

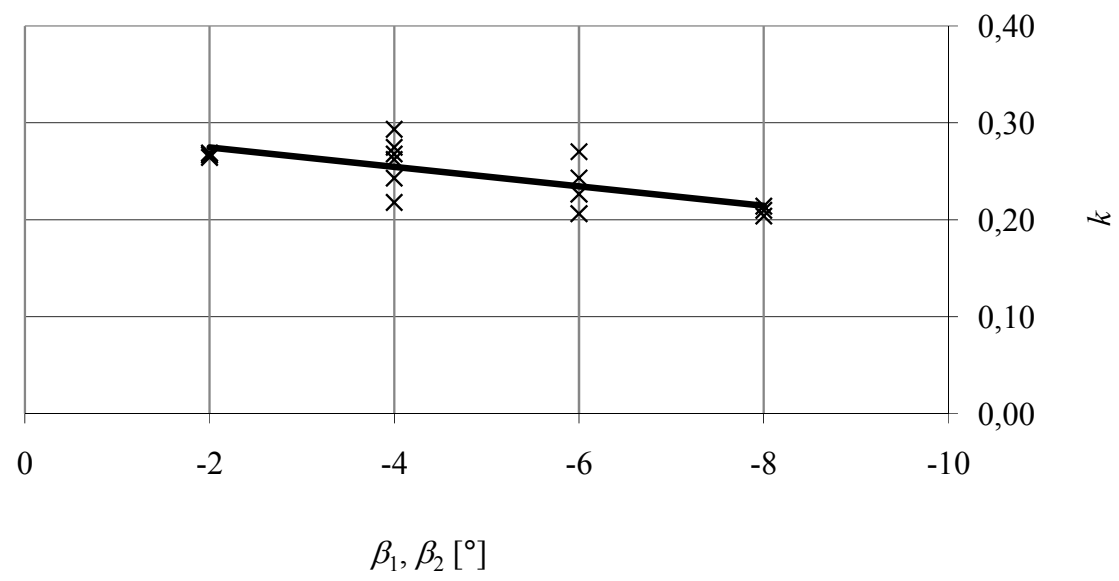

Figura 0.6 Relación de velocidades vs. Paso de las palas. Configuración C05P02 


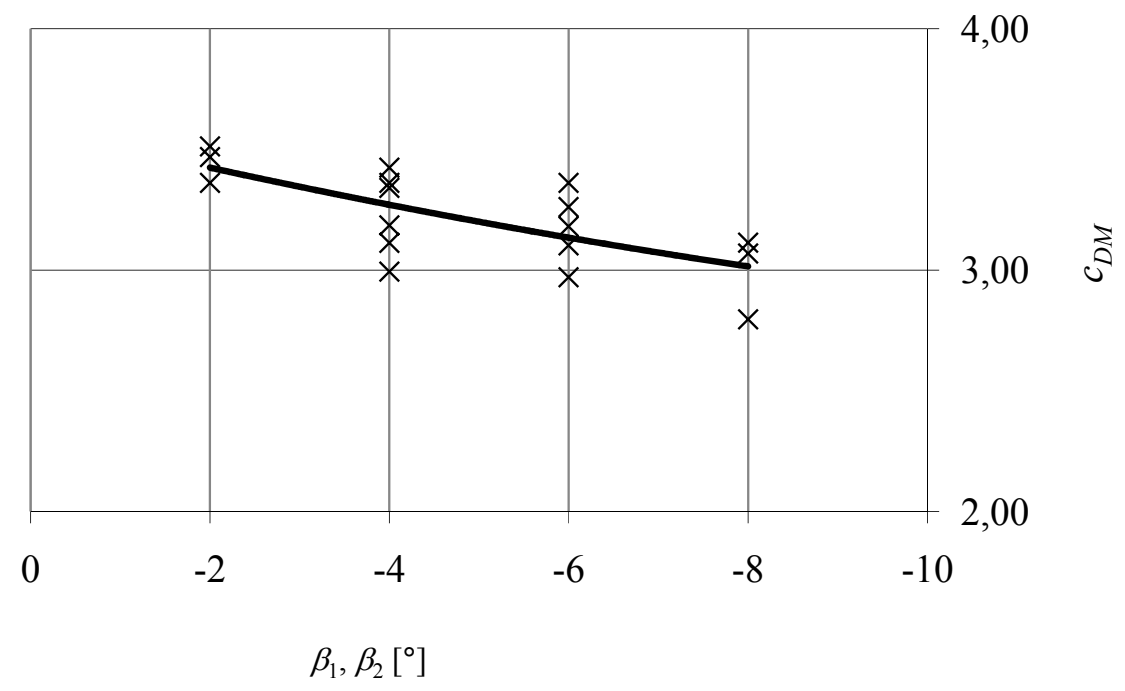

Figura 0.7 Coeficiente de resistencia del modelo vs. Paso de las palas. Configuración C05P02

Si tomamos uno de los ensayos realizados y simulamos el caso de acuerdo a lo desarrollado en el Capítulo 3, será necesario ajustar los parámetros $C_{D}$ y $C_{L \alpha}$ de manera que la solución de equilibrio sea idéntica. Por esta razón el modelo de simulación, por un lado, se verifica y valida cualitativamente, y por otro se ajusta empíricamente para que cuantitativamente tenga la misma respuesta.

El comportamiento cualitativo que indican los parámetros $k$ y $c_{D M}$ con respecto al paso de las palas se valida con las simulaciones numéricas analizadas en el Capítulo 3.

En la Tabla 4.5 se observan las velocidades de traslación y de rotación para las cuales se ajustan los valores de $C_{L \alpha}$ y $C_{D}$ que se muestran en la Tabla 4.6 de manera que las simulaciones numéricas se ajusten cuantitativamente y cualitativamente a los ensayos realizados. 
Tabla 0.6 Ensayos realizados en el túnel vertical y coeficientes aerodinámicos calculados a partir del simulador numérico.

\begin{tabular}{|c|c|c|c|}
\hline Configuración & $\begin{array}{c}\boldsymbol{\beta}_{\boldsymbol{l}}, \boldsymbol{\beta}_{\mathbf{2}} \\
{\left[{ }^{\circ}\right]}\end{array}$ & $\boldsymbol{C}_{\boldsymbol{L} \boldsymbol{\alpha}}$ & $\boldsymbol{C}_{\boldsymbol{D}}$ \\
\hline$C 05 P 02$ & -8 & 1,47 & 0,13 \\
$C 05 P 02$ & -6 & 1,46 & 0,15 \\
$C 05 P 02$ & -4 & 1,40 & 0,15 \\
$C 05 P 02$ & -2 & 1,35 & 0,15 \\
$C 05 P 03$ & -4 & 1,50 & 0,19 \\
$C 05 P 03$ & -6 & 1,40 & 0,09 \\
$C 06 P 02$ & -6 & 1,40 & 0,11 \\
$C 06 P 02$ & -4 & 1,30 & 0,15 \\
$C 06 P 02$ & -2 & 1,40 & 0,19 \\
\hline
\end{tabular}

Es posible observar de la Tabla 4.6 que los coeficientes aerodinámicos de los conjuntos de pala ensayados, para ser usados en el simulador numérico, tienen un valor promedio de $C_{L \alpha}=1,41, C_{D}=0,146$, que resultan congruentes con otros ensayos al respecto (Rosen, A. y Seter, 1991). Utilizando estos valores se obtiene un ajuste numérico del modelo numérico. En la Tabla 4.7 se muestran los valores medidos y los valores obtenidos de la simulación del los parámetros $V_{v} \mathrm{y} \omega$, y el error ponderado de las variables, que se calcula como:

$$
E_{V_{v}, \omega}=\sqrt{\left(\frac{V_{v \text { experimental }}-V_{v \text { simulacion }}}{V_{v \text { experimental }}}\right)^{2}+\left(\frac{\omega_{\text {exp erimental }}-\omega_{\text {simulacion }}}{\omega_{\text {experimental }}}\right)^{2}} 100 \%
$$

Tabla 0.7 Ensayos realizados en el túnel vertical y valores calculados de $N_{e}{ }^{\prime}$ y $k_{e}$.

\begin{tabular}{|c|c|c|c|c|c|c|}
\hline \multirow[b]{2}{*}{ Configuración } & \multirow[b]{2}{*}{$\begin{array}{c}\beta_{1,} \beta_{2} \\
{\left[{ }^{\circ}\right]}\end{array}$} & \multicolumn{2}{|c|}{ Ensayos } & \multicolumn{2}{|c|}{ Simulación } & \multirow[b]{2}{*}{$\mathbf{E}_{\mathbf{V}, \omega}$} \\
\hline & & $\begin{array}{c}\mathbf{V}_{\mathbf{v}} \\
{[\mathrm{m} / \mathrm{s}]}\end{array}$ & $\begin{array}{c}\omega \\
{[\mathrm{rad} / \mathrm{s}]}\end{array}$ & $\begin{array}{c}\mathbf{V}_{\mathbf{v}} \\
{[\mathrm{m} / \mathrm{s}]}\end{array}$ & $\begin{array}{c}\omega \\
{[\mathrm{rad} / \mathrm{s}]}\end{array}$ & \\
\hline$C 05 P 02$ & -8 & 4,92 & 312 & 5,12 & 303 & $5,0 \%$ \\
\hline
\end{tabular}




\begin{tabular}{|c|c|c|c|c|c|c|}
\hline \multirow[b]{2}{*}{ Configuración } & \multirow[b]{2}{*}{$\begin{array}{c}\beta_{1, \infty} \beta_{2} \\
\left.{ }^{0}{ }^{\circ}\right]\end{array}$} & \multicolumn{2}{|c|}{ Ensayos } & \multicolumn{2}{|c|}{ Simulación } & \multirow[b]{2}{*}{$\mathbf{E}_{\mathbf{V}, \omega}$} \\
\hline & & $\begin{array}{c}\mathbf{V}_{\mathbf{v}} \\
{[\mathrm{m} / \mathrm{s}]}\end{array}$ & $\begin{array}{c}\omega \\
{[\mathrm{rad} / \mathrm{s}]}\end{array}$ & $\begin{array}{c}\mathbf{V}_{\mathbf{v}} \\
{[\mathrm{m} / \mathrm{s}]}\end{array}$ & $\begin{array}{c}\omega \\
{[\mathrm{rad} / \mathrm{s}]}\end{array}$ & \\
\hline$C 05 P 02$ & -6 & 4,72 & 298 & 4,79 & 298 & $1,5 \%$ \\
\hline$C 05 P 02$ & -4 & 4,66 & 291 & 4,48 & 294 & $4,0 \%$ \\
\hline$C 05 P 02$ & -2 & 4,58 & 287 & 4,18 & 289 & $8,8 \%$ \\
\hline$C 05 P 03$ & -6 & 3,50 & 260 & 3,52 & 221 & $15,0 \%$ \\
\hline$C 05 P 03$ & -4 & 3,32 & 197 & 3,30 & 218 & $10,7 \%$ \\
\hline$C 06 P 02$ & -6 & 5,50 & 398 & 5,67 & 353 & $11,7 \%$ \\
\hline$C 06 P 02$ & -4 & 5,50 & 356 & 5,03 & 348 & $8,8 \%$ \\
\hline С06P02 & -2 & 5,25 & 316 & 4,96 & 342 & $9,9 \%$ \\
\hline
\end{tabular}

Sobre la evaluación del error ponderado se puede considerar la validación cuantitativa del modelo de simulación numérica.

Como predicen los modelos teóricos y la simulación numérica, los modelos aquí ensayados mostraron un comportamiento estable cuando la relación entre los momentos de inercia principales es $I_{3}>I_{1}, I_{2}$ (Figura 4.6), e inestable cuando $I_{2}>I_{3}>I_{1}$ (Figura 4.7). Este hecho también se verifica en simulaciones numéricas realizadas a tal fin.

Se observa que para configuraciones con ángulo de paso de una pala negativo y el otro positivo, el modelo rota cercano a un eje perpendicular al plano medio entre palas. En la Figura 4.5 se puede ver que el cuerpo del pararrotor se encuentra inclinado y que el eje de simetría rota alrededor de la dirección vertical (eje $Z$ de la terna inercial). Se evidencia de los ensayos que esta inclinación del cilindro aumenta en la medida que la diferencia de paso de las palas, $\delta_{\beta}$, aumenta.

Las simulaciones numéricas muestran idéntico comportamiento, como se observa en la Tabla 3.11 del Capítulo 3. 


\subsubsection{Incertidumbre de las mediciones}

La incertidumbre en las mediciones ha sido evaluada teniendo en cuenta la reproducibilidad de los ensayos realizados en condiciones similares y la incertidumbre de los instrumentos. En la Tabla 4.8 se presentan los valores absolutos de las incertidumbres combinadas (combinación de las desviaciones estándar) en las determinaciones de $k \mathrm{y} c_{D M}, U_{c_{D M}}$ y $U_{k}$, respectivamente, para la Configuración C05P02.

Tabla 0.8 Incertidumbres combinadas en las mediciones para la Configuración C05P02.

\begin{tabular}{|c|c|c|c|c|}
\hline \multicolumn{5}{|c|}{ Incertidumbres combinadas } \\
\hline$\beta$ & $2^{\text {o }}$ & $4^{\text {o }}$ & $6^{\text {o }}$ & $8^{\mathbf{o}}$ \\
\hline$U_{c D M}$ & 0,18 & 0,19 & 0,19 & 0,19 \\
\hline$U_{k}$ & 0,005 & 0,015 & 0,015 & 0,005 \\
\hline
\end{tabular}

\subsection{ENSAYOS EN TÚNEL AERODINÁMICO SOBRE UN MODELO QUE ROTA SOBRE UN RODAMIENTO BASCULANTE}

\subsubsection{Descripción del modelo y configuración experimental}

La configuración experimental se basa en la utilización de un túnel aerodinámico horizontal, donde se realizan los ensayos de la presente serie de modelos físicos (Nadal, Piechocki y Pezzotti, 2010), que se muestra esquemáticamente en la Figura 4.8.

El túnel utilizado es de circuito y cámara de ensayos cerrados, impulsado por una hélice accionada por un motor eléctrico de $37,3 \mathrm{~kW}$. La sección transversal de la cámara de ensayos es rectangular, de 1,4 $\mathrm{m}$ de ancho, 1,0 $\mathrm{m}$ de altura y 7,2 $\mathrm{m}$ de longitud. Es posible hacer variar la velocidad del aire en forma continua hasta $18 \mathrm{~m} / \mathrm{s}$. 


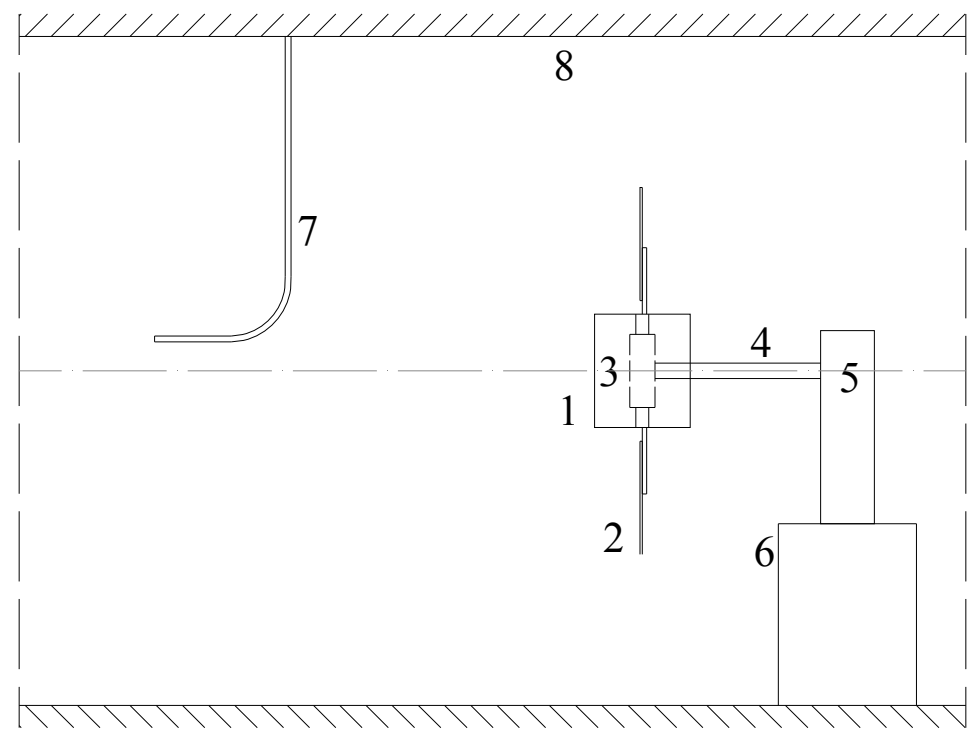

1 Cuerpo principal

2 Palas

3 Rodamiento basculante

4 Brazo

5 Balanza electrónica

6 Soporte

7 Tubo Pitot

8 Tunel de viento

Figura 0.8 Figura esquemática de la configuración de ensayo.

En el interior de sección se ubica el modelo físico a ensayar el cual se fija a una celda de carga por medio de un rodamiento basculante que permite la rotación en 3 grados de libertad, $\omega_{1}, \omega_{2}$ y $\omega_{3}$, e impide el movimiento del pararrotor al respecto de la corriente, que a su vez está alineada con el eje longitudinal del túnel. Si mimetizamos el túnel con el vuelo libre de un pararrotor es posible asimilar el eje longitudinal del túnel de viento con el eje $Z$ inercial. La celda de carga se encuentra vinculada mecánicamente con la estructura del túnel de viento mediante una base rígida.

Los modelos físicos ensayados consisten en un cuerpo principal cilíndrico y dos palas de planta rectangular. Las dimensiones del cilindro son diámetro $90 \mathrm{~mm}$ y altura $92 \mathrm{~mm}$. Las palas planas rectangulares tienen dimensiones $91 \mathrm{~mm}$ de envergadura, $141 \mathrm{~mm}$ de cuerda y 1 mm de espesor; de paso ajustable. Las palas pueden fijarse al cilindro en tres posiciones del eje de las palas respecto al centro de masas del cuerpo a saber:

a) coincidente con el centro de masas,

b) a una distancia de $20 \mathrm{~mm}$ corriente arriba del centro de masas, 
c) a una distancia de $20 \mathrm{~mm}$ corriente abajo del centro de masas,

Estas alternativas de ubicación del plano de las palas será utilizado para ensayar la influencia de la posición de las palas respecto al centro de masas en el comportamiento dinámico del pararrotor. Estas configuran las 3 variantes que se ensayan. En las Figuras 4.09 y Figura 4.10 se muestran vistas generales de los ensayos.

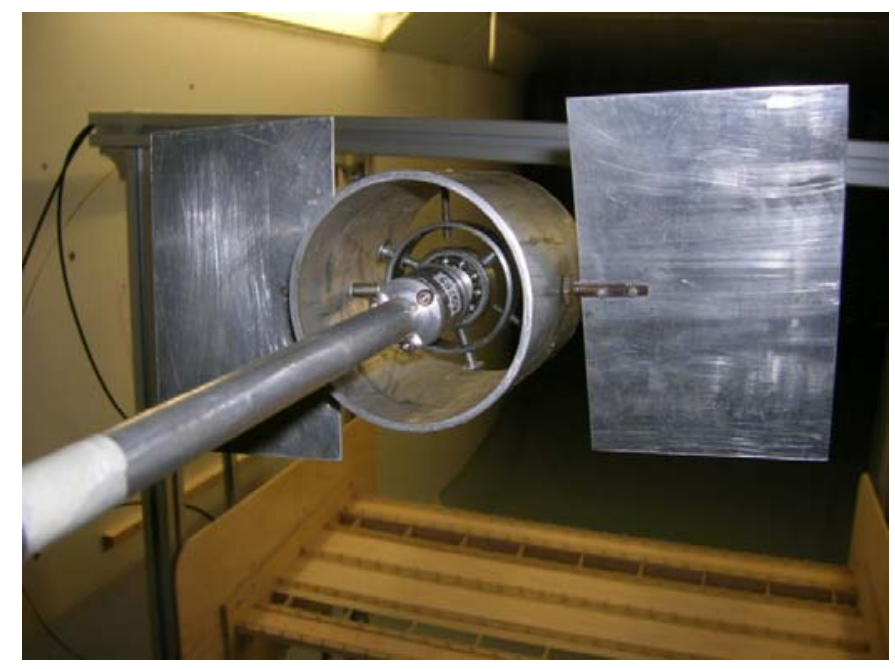

Figura 0.9 Pararrotor montado sobre el rodamiento basculante, sin la tapa sobre el cilindro.

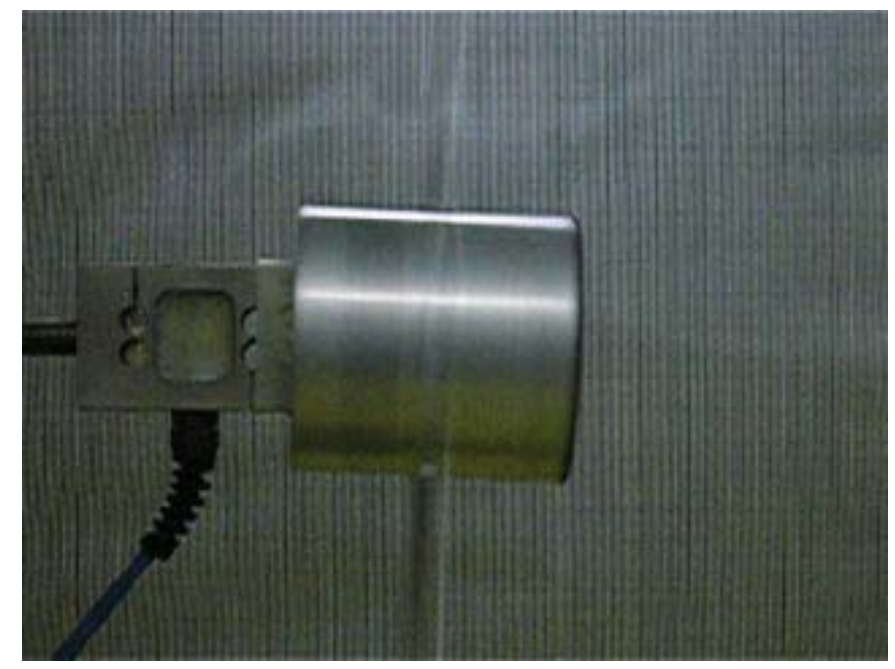


Figura 0.10 Pararrotor montado sobre el rodamiento basculante durante un ensayo. $\beta_{1} \neq \beta_{2}$.

En la Tabla 4.9 se muestran los pesos y momentos de inercia de los distintos modelos ensayados.

Tabla 0.9 Parámetros distintivos de los modelos del pararrotor ensayados.

\begin{tabular}{|c|c|c|c|c|}
\hline$k_{31}$ & $\begin{array}{c}\text { Peso } \\
{[N]}\end{array}$ & $\begin{array}{c}I_{1} \\
{\left[\mathrm{~kg} \mathrm{~m}^{2}\right]}\end{array}$ & $\begin{array}{c}I_{2} \\
{\left[\mathrm{~kg} \mathrm{~m}^{2}\right]}\end{array}$ & $\begin{array}{c}I_{3} \\
{\left[\mathrm{~kg} \mathrm{~m}^{2}\right]}\end{array}$ \\
\hline 0 & 3,5 & $4,61 \cdot 10^{-4}$ & $1,31 \cdot 10^{-3}$ & $1,48 \cdot 10^{-3}$ \\
\hline 0,18 & 4,2 & $5,30 \cdot 10^{-4}$ & $1,56 \cdot 10^{-3}$ & $1,66 \cdot 10^{-3}$ \\
\hline$-0,18$ & 4,2 & $5,30 \cdot 10^{-4}$ & $1,56 \cdot 10^{-3}$ & $1,66 \cdot 10^{-3}$ \\
\hline
\end{tabular}

\subsubsection{Parámetros medidos e instrumentación}

En los ensayos se han medido los siguientes parámetros:

- velocidad de rotación, por medio de un tacómetro láser,

- paso de las palas, por medio de un goniómetro,

- velocidad de la corriente, por medio de un tubo Pitot estandarizado y un micromanómetro,

- resistencia aerodinámica del modelo, por medio de una celda de carga,

- peso del modelo, por medio una balanza electrónica,

- ángulo de nutación promedio, por medio de fotografía convencional sincronizada, ubicada a sobre dos ejes perpendiculares transversales al eje del túnel, sobre un registro de fondo,

- temperatura ambiente, mediante un termómetro de bulbo húmedo y bulbo seco, 
- presión barométrica, mediante un barómetro electrónico.

El instrumental utilizado se detalla en la Tabla 4.2.

\subsubsection{Definiciones}

Idénticamente al Capítulo 2 y 3 se define el parámetro $k_{31}$ como la relación entre la distancia del plano de las palas al centro de masas y coordenada según el eje de las palas del centro de presiones de la pala, siendo este parámetro positivo cuando las palas están situadas corriente arriba del centro de masas.

\subsubsection{Ensayos realizados y resultados}

En la Tabla 4.10 se presentan las configuraciones ensayadas, así como también los valores medios de los parámetros $k, c_{D M}, \mathrm{y}$ del ángulo de nutación, $\theta_{e}$.

Tabla 0.10 Valores medios de los parámetros medidos de distintas configuraciones de pararrotor.

\begin{tabular}{|c|c|c|c|c|c|c|c|c|}
\hline $\boldsymbol{k}_{\mathbf{3 1}}$ & $\begin{array}{c}\boldsymbol{\beta}_{\mathbf{1}} \\
\text { [grados] }\end{array}$ & $\begin{array}{c}\boldsymbol{\beta}_{\mathbf{2}} \\
\text { [grados] }\end{array}$ & $\begin{array}{c}\boldsymbol{\delta}_{\boldsymbol{\beta}} \\
\text { [grados] }\end{array}$ & $\begin{array}{c}\boldsymbol{\varepsilon} \\
\text { [grados] }\end{array}$ & $\mathbf{1 0} \mathbf{R e}$ & $\boldsymbol{k}$ & $\boldsymbol{C}_{\boldsymbol{D M}}$ & $\begin{array}{c}\boldsymbol{\theta}_{\boldsymbol{\varepsilon}} \\
\text { [grados] }\end{array}$ \\
\hline 0 & -4 & -4 & 0 & -8 & 250 & 0,27 & 3,22 & 0,18 \\
\hline 0 & -4 & -8 & 2 & -12 & 293 & 0,25 & 3,44 & 1,65 \\
\hline 0 & -4 & -12 & 4 & -16 & 286 & 0,27 & 3,44 & 2,06 \\
\hline$-0,18$ & -4 & -4 & 0 & -8 & 207 & 0,31 & 3,47 & 0,08 \\
\hline$-0,18$ & -4 & -8 & 2 & -12 & 262 & 0,30 & 3,32 & 4,75 \\
\hline$-0,18$ & -4 & -12 & 4 & -16 & 252 & 0,26 & 3,43 & 6,22 \\
\hline 0,18 & -4 & -4 & 0 & -8 & 296 & 0,26 & 3,55 & 0,51 \\
\hline 0,18 & -4 & -8 & 2 & -12 & 265 & 0,24 & 3,36 & 3,08 \\
\hline 0,18 & -4 & -12 & 4 & -16 & 300 & 0,26 & 3,19 & 6,37 \\
\hline
\end{tabular}


En la Figuras 4.11 y la Figura 4.12 se ve la variación del ángulo de nutación con la posición de las palas, el ángulo de paso y la relación de velocidades.

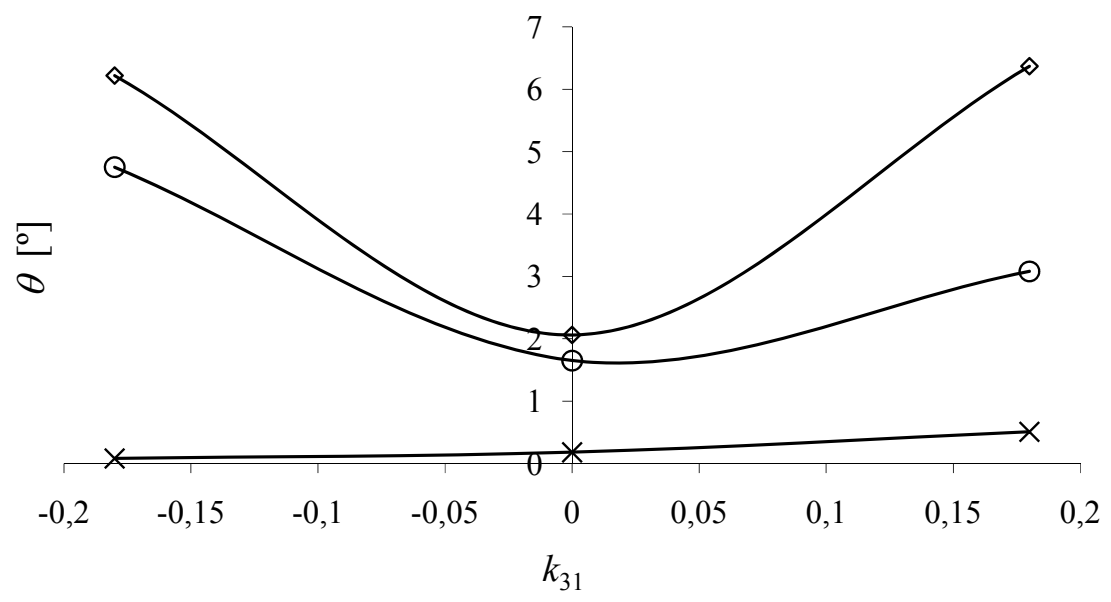

Figura 0.11 Ángulo de nutación para $\beta_{1}=-4^{\circ}$ y $\beta_{2}=-8^{\circ}$. Los círculos corresponden a $k_{31}=0$; los cuadrados, a $k_{31}=0,17 ; \mathrm{y}$ los triángulos, a $k_{31}=-0,17$

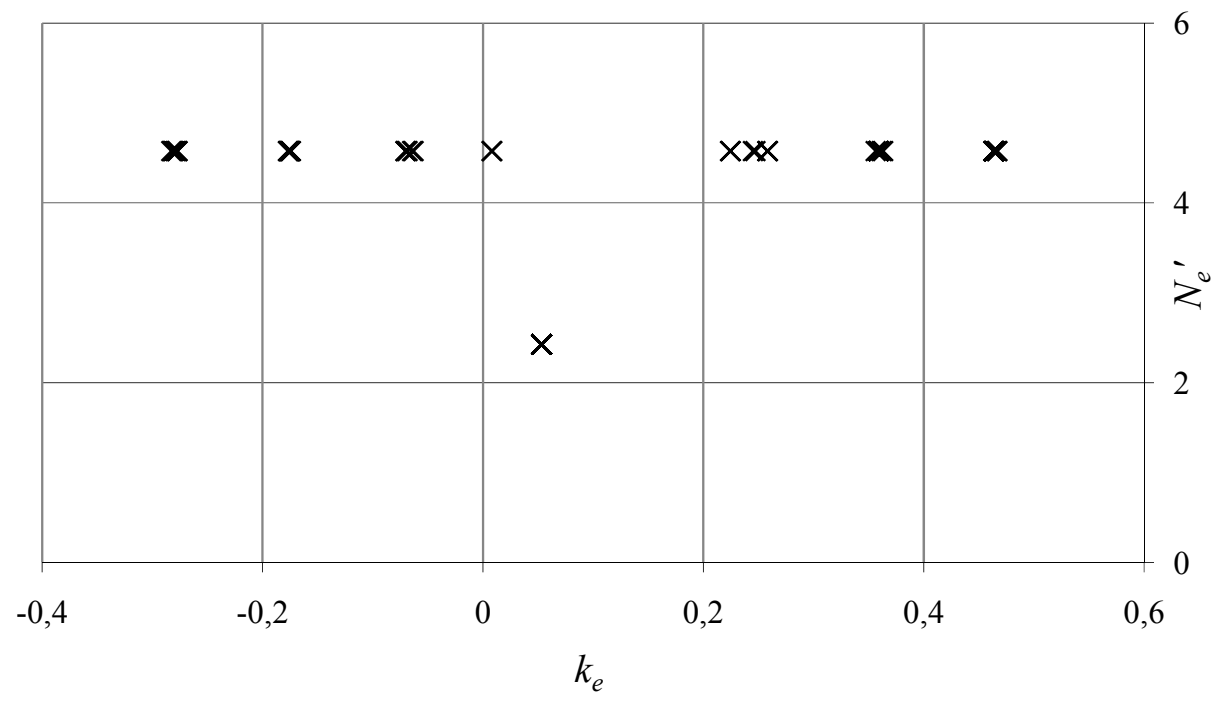


Figura 0.12 Gráfico $\left(N_{e}, k_{e}\right)$ para los ensayos realizados.

Se puede apreciar en los ensayos realizados que en la totalidad de los casos la solución de equilibrio se encuentra cercana al ángulo de nutación nulo (solución Tipo I).

\subsubsection{Análisis de los resultados}

De los ensayos realizados se puede observar que $k$ tiende a disminuir levemente al aumentar $\delta_{\beta}\left(\mathrm{y} \beta_{0}\right)$, como se ve en la Figura 4.13. Esta conclusión es congruente con la encontrada en los ensayos de túnel vertical.

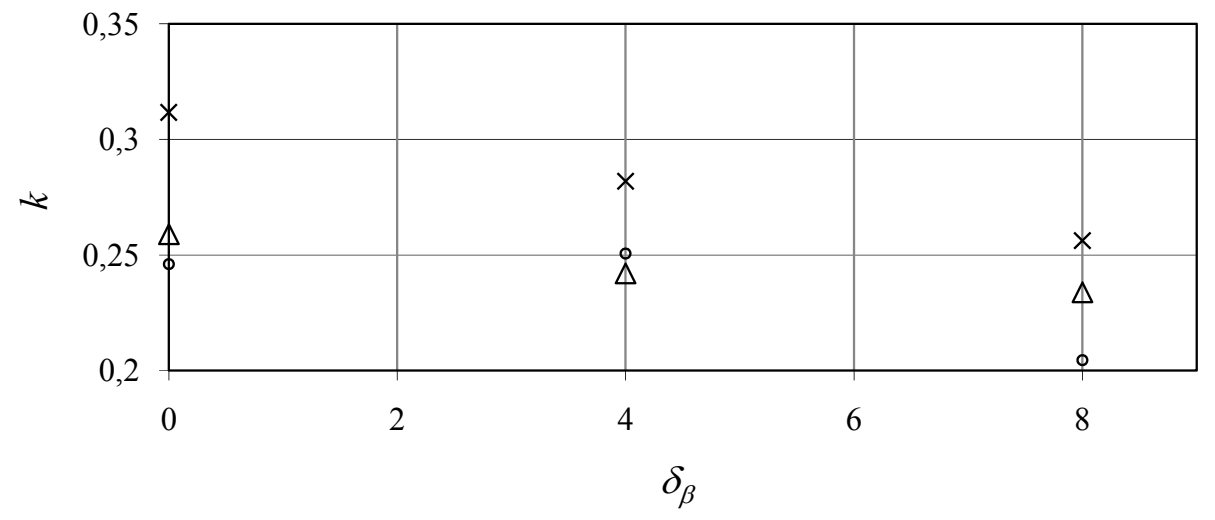

Figura 0.13 Parámetro $k$ en función de la diferencia de paso entre las palas $\delta_{\beta}$, para distintos valores de $k_{31}$. De los símbolos utilizados para indicar los puntos ensayados, los círculos corresponden a $k_{31}=0$, las cruces a $k_{31}=-1$ y los triángulos a $k_{31}=1$.

Se puede observar en la Figura 4.11 que el ángulo de nutación de la solución de equilibrio, $\theta_{e}$, aumenta con el valor absoluto de $k_{31}$. También se puede ver que existe un comportamiento del pararrotor al respecto del ángulo de nutación aproximadamente simétrico alrededor de $k_{31}$ $=0$, en coincidencia con lo que se desprende de las simulaciones numéricas para pequeños valores de $k_{31}$. Para $\delta_{\beta}=0$ se observa que el ángulo de nutación de equilibrio no es claramente 
diferenciable del valor nulo. Finalmente un aumento de $\delta_{\beta}$ introduce un aumento del ángulo de nutación de equilibrio.

No se observan patrones definidos en el comportamiento del coeficiente de resistencia del modelo para estos ensayos.

El pararrotor se comportó en forma estable para todos los casos ensayados.

\subsubsection{Incertidumbre de las mediciones}

La incertidumbre en las mediciones ha sido evaluada teniendo en cuenta la reproducibilidad de los ensayos realizados en condiciones similares y la incertidumbre de los instrumentos. Los valores absolutos de las incertidumbres combinadas (combinación de las desviaciones estándar) en las determinaciones de $k$ y $c_{D M}$, para el caso $\beta_{1}=\beta_{2}=-4^{\circ}, k_{31}=0$, son $U_{c_{D M}}=0,24$ y $U_{k}=0,002$, respectivamente.

La incertidumbre en las mediciones de ángulo de nutación ha sido evaluada a partir de la determinación de la incertidumbre de resolución de las imágenes capturadas de los ensayos sobre las que se mide $\theta$, y la incertidumbre de las observaciones realizadas (la cual es consecuencia del análisis estadístico de las muestras tomadas), resultando la incertidumbre combinada de la medición del ángulo de nutación de $1,3^{\circ}$.

\subsection{ENSAYOS REALIZADOS EN TRABAJOS DE REFERENCIA}

Se comprueba en la bibliografía de referencia que existen mediciones experimentales debidamente documentadas donde el modelo y configuración experimental se encuentra lo suficientemente especificada como para permitir simular numéricamente estos experimentos. Uno de estos casos es el estudio efectuado por Kellas (2007) donde se comparan resultados de 
una rutina de simulación numérica y ensayos en vuelo libre para un decelerador aerodinámico tipo sámara, similar a un pararrotor de ala única.

\subsubsection{Descripción del modelo y configuración experimental}

La configuración experimental usada en el documento de referencia se basa en el vuelo libre, atmosférico, de modelo instrumentado que imita en su concepción a las sámaras de las semillas de Arce. El modelo físico se muestra esquemáticamente en la Figura 4.14.

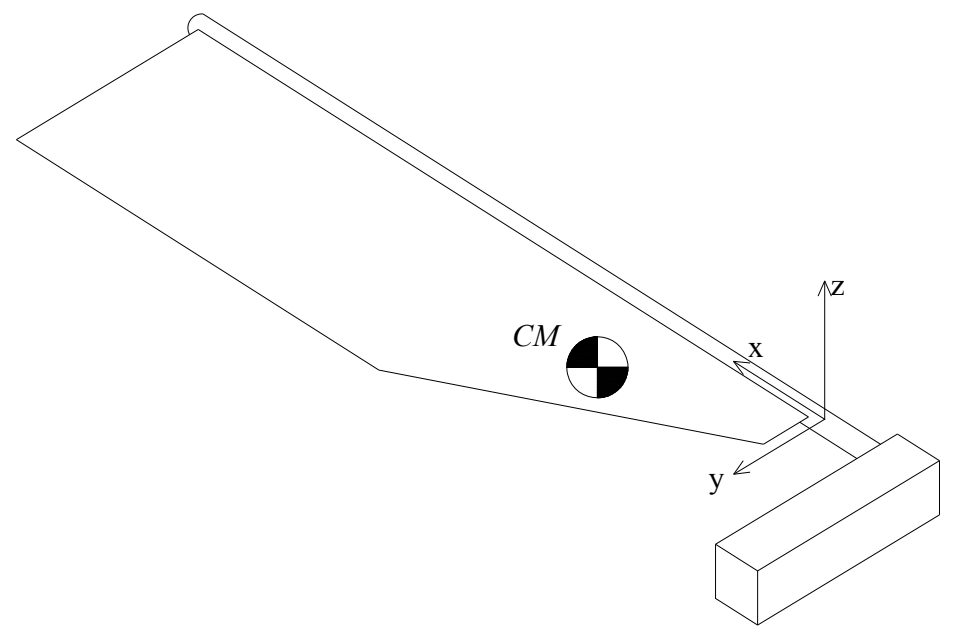

Figura 0.14 Esquema del modelo físico de sámara

En particular el modelo ensayado que se adopta presenta las características geométricas y físicas que se transcriben en la Tabla 4.11.

Tabla 0.11 Valores transcriptos del trabajo de A. Kellas (2007) al respecto del modelo físico de sámara considerado

\begin{tabular}{|c|c|}
\hline Parámetro & Valor \\
\hline Peso & $0,31 \mathrm{~kg}$ \\
\hline
\end{tabular}




\begin{tabular}{|c|c|}
\hline Parámetro & Valor \\
\hline Coordenadas del centro de masas & {$[0,0203 ; 0,0553 ; 0,0039]$} \\
\hline Cuerda de la pala & $0,74 \mathrm{~m}$ \\
\hline Envergadura de la pala & $0,15 \mathrm{~m}$ \\
\hline$I_{\mathrm{x}}$ & $2,229 \cdot 10^{-4} \mathrm{~kg} \mathrm{~m}^{2}$ \\
\hline$I_{\mathrm{y}}$ & $9,930 \cdot 10^{-3} \mathrm{~kg} \mathrm{~m}^{2}$ \\
\hline$I_{\mathrm{z}}$ & $1,010 \cdot 10^{-3} \mathrm{~kg} \mathrm{~m}^{2}$ \\
\hline$I_{\mathrm{yx} ;} I_{\mathrm{xy}}$ & $-5,860 \cdot 10^{-5} \mathrm{~kg} \mathrm{~m}^{2}$ \\
\hline$I_{\mathrm{xz}} ; I_{\mathrm{zx}}$ & $5,690 \cdot 10^{-7} \mathrm{~kg} \mathrm{~m}^{2}$ \\
\hline$I_{\mathrm{yz}} ; I_{\mathrm{zy}}$ & $-1,170 \cdot 10^{-5} \mathrm{~kg} \mathrm{~m}^{2}$ \\
\hline
\end{tabular}

A los fines de la implementación de la simulación numérica descripta en el Capítulo 3 es necesario definir la posición espacial del centro de presiones sobre el ala. Al respecto es posible inferir de los datos volcados en la Tabla 4.11 que $r_{11}=0,4318 \mathrm{~m}$ para una posición al $60 \%$ de la envergadura y $r_{13}=0,0039 \mathrm{~m}$. No obstante el parámetro $r_{12}$, posición del centro de masas en la dirección de la cuerda del ala necesita ser determinado experimentalmente. El trabajo de A. Kellas (2007) menciona la relevancia de este parámetro al respecto del desempeño y estabilidad del dispositivo, y su variabilidad dependiendo del ángulo de ataque.

\subsubsection{Parámetros medidos e instrumentación}

En los ensayos que se mencionan en el documento de referencia, se han medido:

- velocidad de rotación,

- velocidad de la corriente, 
- ángulo de inclinación del modelo, equivalente al ángulo de nutación.

\subsubsection{Ensayos realizados y resultados}

Los ensayos realizados arrojan los resultados al respecto de la solución de equilibrio que se muestran en la Tabla 4.12, que se transcriben del documento de referencia.

Tabla 0.12 Valores transcriptos del trabajo de Kellas (2007) al respecto del desempeño medido del modelo físico de sámara considerado.

\begin{tabular}{|c|c|}
\hline Parámetro & Valor \\
\hline Rotación propia & $17,1 \mathrm{rad} / \mathrm{s}$ \\
\hline Velocidad de descenso & $2,8 \mathrm{~m} / \mathrm{s}$ \\
\hline $\begin{array}{c}\text { Ángulo de inclinación } \\
\text { (nutación) }\end{array}$ & $0,524 \mathrm{rad}\left(30,0^{\circ}\right)$ \\
\hline Ángulo de paso de pala & $0,1 \mathrm{rad}$ \\
\hline
\end{tabular}

Se observa de los resultados de los ensayos realizados que la sámara desciende en equilibrio con un ángulo de nutación constante, describiendo un movimiento cónico dado por su ángulo de inclinación.

\subsubsection{Comparativa con la simulación numérica y resultados}

Con la configuración presentada en el documento de referencia se configuró la simulación numérica presentada en el Capítulo 3 de manera que represente su comportamiento dinámico en vuelo, de acuerdo a los lineamientos discutidos en el capítulo anterior.

El simulador numérico fue adaptado a los fines de este apartado para considerar matrices de inercia no diagonales, en vista que la terna fija a la sámara no está según los ejes de inercia principales.

Para la simulación numérica se realizaron las siguientes consideraciones: 
- los valores de $C_{L \alpha}$ y $C_{D}$ son los correspondientes a los de una placa plana, indicados en NACA - TN 3221, para el ángulo de ataque correspondiente,

- el valor de posición del centro de presiones en dirección de la cuerda se determina de manera que el desempeño en términos de velocidad de descenso y rotación sobre el eje vertical resulte coincidente entre la simulación numérica y los ensayos.

Los valores adoptados resultan entonces:

- $C_{L \alpha}=2$, para un ángulo de ataque que se desprende de la simulación numérica de 0,36 $\mathrm{rad}$,

- $C_{D}=0,27$, para un ángulo de ataque que se desprende de la simulación numérica de $0,36 \mathrm{rad}$,

$-r_{12}=0,0021$

Por otro lado la solución de equilibrio que se configura como resultado de la simulación numérica se encuentra dada por:

$\omega_{1}=8,67 \mathrm{rad} / \mathrm{s}$

$\omega_{2}=1,94 \mathrm{rad} / \mathrm{s}$

$\omega_{3}=17,05 \mathrm{rad} / \mathrm{s} ;$

$U=2,83 \mathrm{~m} / \mathrm{s}$.

El comportamiento de los ángulos de Euler resulta:

$d \theta / d t=0, \theta=0,48 \mathrm{rad}$

$d \varphi / d t=0, \psi=1,35 \mathrm{rad}$

$d \psi / d t=19,23 \mathrm{rad} / \mathrm{s}$. 
La simulación numérica arroja como resultado que el ángulo de rotación propia es constante y que la precesión aumenta a una velocidad angular cercana a $\omega_{3}$. Vale decir que su configuración de vuelo en equilibrio es describiendo un cono de ángulo igual al de nutación a una velocidad cercana a $\omega_{3}$.

Para estas condiciones el ángulo de nutación de equilibrio que resulta de la simulación es de 0,48 radianes, un $7,6 \%$ menor que el ángulo medido en los ensayos que resulta de 0,52 radianes.

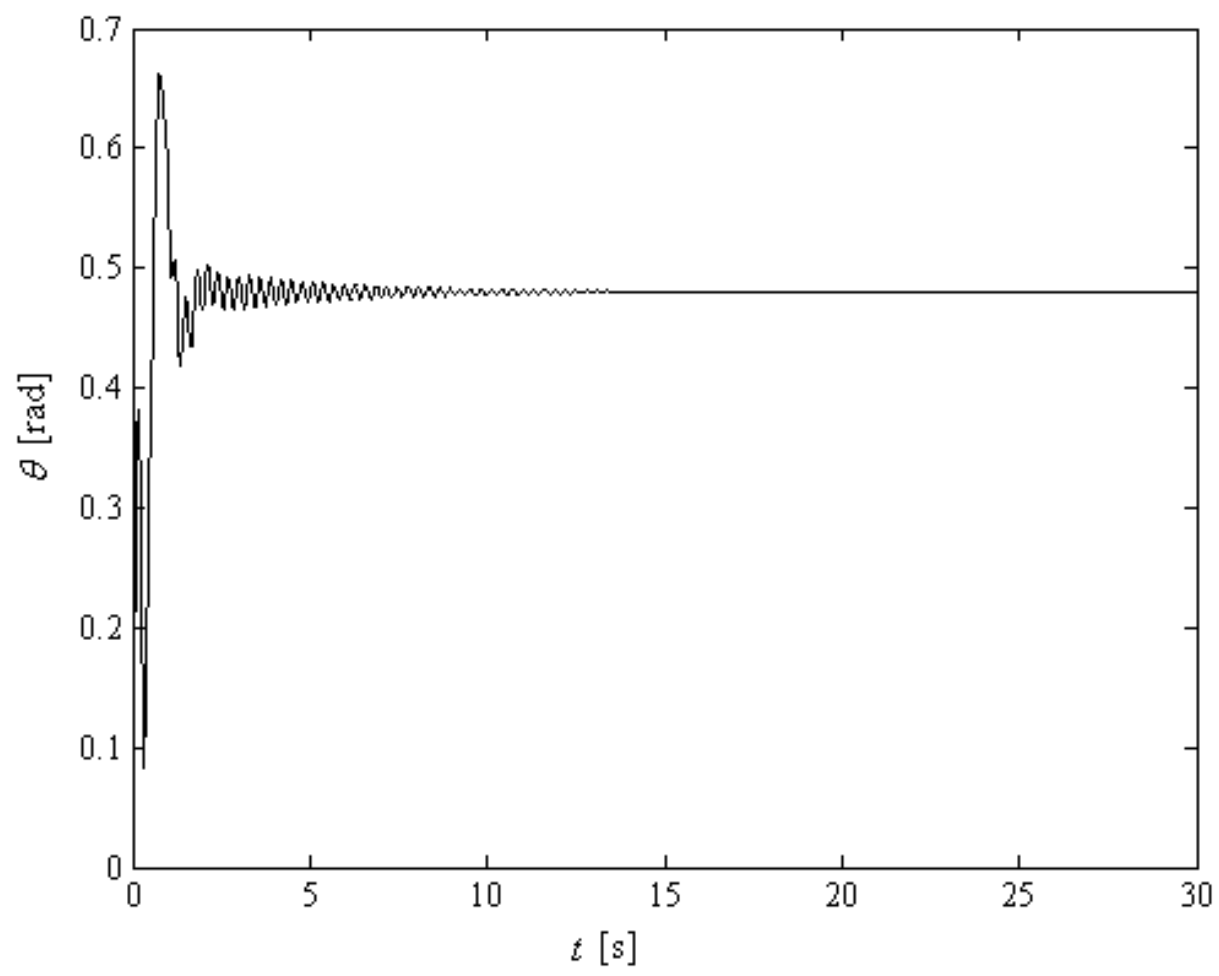

Figura 0.15 Evolución del ángulo de nutación $\theta$ con el tiempo, para la simulación numérica de la sámara

Si consideramos la información de origen experimental que suministra el trabajo de referencia y la simulación numérica realizada, presentada en la Tabla 4.13, es posible observar que la simulación reproduce correctamente la situación experimental. Por otro lado 
se observa que la solución de equilibrio de los ensayos experimentales muestra una velocidad angular de precesión cercana a los valores de la velocidad de rotación, y ángulos de nutación y rotación propia constantes, lo cual es coincidente con lo arrojado por la simulación numérica.

Tabla 0.13 Comparativa entre la solución de equilibrio que arroja la simulación numérica y los experimentales transcriptos del trabajo de Kellas (2007).

\begin{tabular}{|c|c|c|c|}
\hline Parámetro & $\begin{array}{c}\text { Simulación } \\
\text { numérica }\end{array}$ & $\begin{array}{c}\text { Vuelo libre } \\
\text { (Kellas, 2007) }\end{array}$ & Error porcentual \\
\hline$\omega_{3}$ & $17,05 \mathrm{rad} / \mathrm{s}$ & $17,07 \mathrm{rad} / \mathrm{s}$ & $0,1 \%$ \\
\hline$U$ & $2,83 \mathrm{~m} / \mathrm{s}$ & $2,8 \mathrm{~m} / \mathrm{s}$ & $0,1 \%$ \\
\hline$\theta_{e}$ & $0,48 \mathrm{rad}$ & $0,52 \mathrm{rad}$ & $7,6 \%$ \\
\hline
\end{tabular}

\subsubsection{Conclusiones}

Los ensayos realizados por Kellas (2007) validan la capacidad de describir la actitud del pararrotor por parte del simulador numérico.

Por otro lado también se puede concluir que es posible pensar configuraciones de pararrotor con grandes ángulos de ataque donde el flujo se encuentre totalmente desprendido de la superficie de las alas como sucede en las determinaciones de Heyson y Boswinke (1955), que son confirmadas por los ensayos realizados por Crimi (1988). Para los coeficientes aerodinámicos previstos en estos trabajos anteriores, la simulación estima satisfactoriamente desempeño y actitud.

\subsection{COMPARACIÓN DE LOS RESULTADOS DE LOS DIFERENTES ENSAYOS}

Se han seleccionado los parámetros $k$ y $c_{D M}$ para la comparación de los resultados de los diferentes ensayos. Estos valores se presentan en la Tabla 4.14. 
Tabla 0.14 Valores de $k$ y $c_{D M}$ para los diferentes ensayos.

\begin{tabular}{|c|c|c|c|c|c|c|c|c|c|c|}
\hline \multirow{3}{*}{ Ensayo } & \multirow{3}{*}{ Solidez } & \multirow{3}{*}{$10^{-3} \mathrm{Re}$} & \multicolumn{4}{|c|}{$\boldsymbol{k}$} & \multicolumn{4}{|c|}{$c_{D M}$} \\
\hline & & & \multicolumn{4}{|c|}{$\beta_{1}, \beta_{2}$ [grados] } & \multicolumn{4}{|c|}{$\beta_{1}, \beta_{2}$ [grados] } \\
\hline & & & -2 & -4 & -6 & -8 & -2 & -4 & -6 & -8 \\
\hline Eje fijo $A^{*}$ & 0,45 & $57-300$ & 0,42 & 0,34 & 0,31 & 0,28 & 3,43 & 3,35 & 3,35 & 3,00 \\
\hline Túnel vertical & 0,52 & $40-75$ & 0,27 & 0,26 & 0,24 & 0,21 & 3,44 & 3,24 & 3,18 & 2,99 \\
\hline Basculante & 0,36 & 250 & - & 0,27 & - & - & - & 3,22 & - & - \\
\hline
\end{tabular}

* Nadal Mora, 2005.

Se observa que el pararrotor para los ensayos con eje fijo gira más lento que para los restantes ensayos; esto presumiblemente se debe a la fricción de los dos rodamientos que sostienen al modelo.

Respecto a los valores del coeficiente de resistencia, no se observan variaciones significativas.

No se observan diferencias cualitativas en el comportamiento del pararrotor entre los ensayos realizados.

\subsection{CONCLUSIONES}

Se ha estudiado experimentalmente el comportamiento dinámico de una configuración de decelerador aerodinámico. Se han realizado dos tipos diferentes de ensayos que complementan aquellos realizados por Nadal Mora (2005). Cada uno ha permitido observar el comportamiento dinámico del pararrotor y medir los parámetros que caracterizan su solución de equilibrio, como $k$ y $c_{D M}$, que son comunes a todos los ensayos realizados. 
Es posible encontrar valores medios o funciones de los coeficientes aerodinámcos que caracterizan a las palas de manera que el simulador numérico reproduzca los ensayos realizados.

Se convalida a partir de los ensayos en el túnel vertical lo predicho por el simulador al respecto de la disminución de la relación de velocidades $k$ y del coeficiente de resistencia del modelo $c_{D M}$ al aumentar el ángulo de paso de las palas. Este hecho indica que los valores máximos de $c_{D M}$ se alcanzan a ángulos mínimos de $\beta_{1} \mathrm{y} \beta_{2}$.

Se confirma a partir de los ensayos realizados que es posible obtener soluciones estables bajo la condición $I_{3}>I_{2}, I_{1}$. Las configuraciones ensayadas, donde $I_{2}>I_{3}, I_{1}$, fueron inestables. Las simulaciones numéricas arrojan idéntica respuesta al respecto de la estabilidad del sistema para las condiciones medidas en los ensayos.

El comportamiento del ángulo de nutación es congruente entre ambos ensayos y validan los resultados del simulador numérico. Las soluciones de estabilidad del vuelo del pararrotor tienen asociado un ángulo dado de nutación, aproximadamente constante, que representa la inclinación del eje 3 con respecto del eje inercial $Z$.

Se comprueba de los ensayos realizados que el ángulo de nutación aumenta al aumentar los valores absolutos de $\delta_{\beta}$ y $k_{31}$. Este efecto se verifica en las simulaciones numéricas. También es posible observar que para $\delta_{\beta}=0$ el ángulo de nutación de la solución de equilibrio es aproximadamente cero, y que un aumento de $\delta_{\beta}$ implica la aparición de soluciones de equilibrio con ángulos de nutación muy pequeños, denominados solución Tipo I en el Capítulo 3. 


\section{CAPÍTULO 5}

\section{CONCLUSIONES}

\subsection{INTRODUCCIÓN}

En el presente capítulo se presenta un resumen de las conclusiones recogidas en cada capítulo componente de la presente tesis, su interrelación, sus aportes novedosos y las futuras líneas de investigación vinculadas a la temática abordada.

Si bien existe abundante información acerca del uso práctico de dispositivos asimilables al pararrotor estudiado, no existen estudios específicos, previos al presente trabajo, que den cuenta de modo general de los efectos de los parámetros de diseño sobre la estabilidad, comportamiento dinámico y desempeño como desacelerador. Estos estudios son de vital importancia para su utilización de manera controlada.

\subsection{MODELO ANALÍTICO}

El modelo analítico se ha desarrollado sobre la base de hipótesis simplificadoras y la linealización del sistema resultante, tomando como punto de partida el trabajo de Nadal Mora (2005). Este modelo arroja como conclusión que la estabilidad del movimiento del pararrotor se encuentra definida por dos parámetros adimensionales que contienen la relación de los momentos de inercia, la geometría y las características aerodinámicas del dispositivo.

En esta Tesis se ha desarrollado el caso donde el eje que contiene a las palas no pasa por el centro de masas del pararrotor. Llamaremos centro de sustentación al punto de intersección del eje que contiene a las palas con el eje de simetría cilíndrico del pararrotor. La distancia 
del centro de sustentación al centro de masas está dada por el parámetro adimensional $k_{31}$. Al respecto se ha encontrado que, para pequeños desplazamientos del centro de sustentación, la estabilidad del pararrotor (según el modelo linealizado del sistema) no se ve afectada por el parámetro $k_{31}$, respecto del caso en que coincida con el centro de masas, bajo las hipótesis del modelo y las simplificaciones realizadas teniendo en cuenta los órdenes de magnitud de cada término. Para desplazamientos del orden de la distancia desde el centro de presiones de las palas al eje de revolución del pararrotor o mayores, estos sí influyen en la estabilidad dinámica del dispositivo.

En los casos en que los momentos de inercia cumplen las condiciones $I_{3}>I_{2}, I_{1}$ y $I_{3}<I_{2}, I_{1}$ es posible obtener configuraciones estables del desacelerador aerodinámico. En ambos casos valores positivos del parámetro $k_{31}$ (centro de masas por debajo del centro de sustentación) contribuyen a la estabilidad del sistema. En el caso que se esté cerca del límite estabilidad del sistema el parámetro $k_{31}$ resulta definitorio de la estabilidad.

En el caso en que $I_{2}>I_{3}>I_{1}$, el pararrotor se comporta de manera inestable, sin que los efectos del parámetro $k_{31}$ resulten determinantes.

En el caso en que $I_{1}>I_{3}>I_{2}$, el pararrotor se comporta en general de manera inestable. No obstante es posible teóricamente alcanzar zonas de estabilidad para valores muy grandes de $k_{31}$.

En el caso que $I_{3}<I_{2}, I_{1}$ el parámetro $k_{31}$ contribuye a la estabilidad tanto si este es mayor que cero como si este es menor que cero.

Se observa a partir del desarrollo de modelo analítico que el parámetro $k_{31}$ podría utilizarse como parámetro de control del sistema, al igual que la diferencia entre los ángulos de paso de las palas. Este hecho se verifica al observar los términos independientes de la linealización del modelo matemático del sistema (40) y (42), que incluyen estos parámetros. Su actuación podría utilizarse para el guiado del dispositivo con velocidades de respuesta controladas. En cualquier caso ambos parámetros resultan parámetros de diseño determinantes. 
El modelo analítico permite determinar los ángulos de nutación $\theta$ y de rotación propia $\varphi$ de acuerdo con la geometría mostrada en la Figura 2.2 de idéntica manera a lo desarrollado para $k_{31}=0$ por Nadal Mora (2005), sobre la base de la descomposición de la velocidad angular en coordenadas sobre la terna móvil. La determinación de ángulos de nutación $\theta$ y de rotación propia $\varphi$ por medio de este modelo, se encuentra referida a un tipo de solución, denominada Tipo I, donde el ángulo de nutación es de pequeña magnitud, que es la solución estable que predice el modelo analítico.

Se introduce en el desarrollo del modelo analítico dos parámetros, $N_{e}{ }^{\prime}$ y $k_{e}$, basados en aquellos introducidos por Nadal Mora (2005), que permiten caracterizar la respuesta dinámica del sistema estableciendo regiones donde se inscriben los casos estudiados.

\subsection{MODELO DE SIMULACIÓN NUMÉRICA}

El modelo de simulación numérica permite el estudio del sistema dinámico sin las hipótesis simplificativas y linealizaciones que fueron adoptadas en el modelo analítico. Las simulaciones numéricas muestran, cualitativamente, la existencia de tres tipos de soluciones. El primer tipo de solución, denominada Tipo I, se caracteriza porque el ángulo de nutación de equilibrio es 0 , o muy cercano a 0 si $\delta_{\beta} \neq 0$, que es la solución estable que se halló a partir del modelo analítico. El segundo tipo de solución (denominada Tipo II) se caracteriza porque el ángulo de nutación de equilibrio es mucho mayor que 0 grados, que se aleja de lo predicho por el modelo analítico, implicando una actitud de vuelo del pararrotor con el eje 3 permanentemente inclinado al respecto al eje inercial Z Z. La posición del punto de bifurcación que da paso a esta solución depende de la relación de momentos de inercia, del valor de $k_{31}$, de $\delta_{\beta}, \mathrm{y}$ de la relación $C_{L \alpha} / C_{D}$, principalmente. En el tercer caso, la solución es dinámicamente inestable.

De los casos simulados se observa que para determinados valores de coeficientes aerodinámicos de las palas, el modelo analítico y la simulación numérica son congruentes en la estimación de la solución de equilibrio, cuando esta es de la denominada Tipo I. Los 
resultados de las simulaciones y del modelo analítico son congruentes y el error entre ellos es de pequeña magnitud (menor a un 10\%).

Un aumento en el valor absoluto de $k_{31}$ hace que la bifurcación que da paso a las soluciones Tipo II aparezca a menores valores de $\delta_{\beta}$.

Las soluciones de equilibrio encontradas en las simulaciones introducen la existencia de soluciones con ángulo de nutación mucho mayor a 0 , no previstas en el modelo analítico, como solución de transición entre las Tipo I y las soluciones inestables.

Con las simulaciones numéricas se ha comprobado la predicción del modelo analítico de la existencia de regiones en el gráfico $N_{e}, k_{e}$ que permiten resumir el comportamiento dinámico y la estabilidad del sistema. Este resultado permite economizar en gran medida el estudio de la estabilidad de los pararrotores, en cuya configuración hay que tener en cuenta un gran número de parámetros, que así quedan reducidas sólo a dos parámetros adimensionales.

Se pueden destacar los siguientes resultados:

a. Las simulaciones numéricas muestran el comportamiento del pararrotor incluyendo efectos no lineales y efectos de órdenes de magnitud menores no contemplados en el modelo analítico.

b. Se hallan tres tipos de regiones coincidentes con las soluciones que se pueden encontrar, que incluyen las soluciones Tipo I, Tipo II e inestables.

c. Al igual que las conclusiones sacadas a partir del modelo analítico, la relación entre momentos de inercia principales resultan definitorios para la dinámica del sistema simulado numéricamente. Por un lado, como en el modelo analítico, es posible clasificar su comportamiento según los denominados Caso A $\left(I_{3}>I_{2}, I_{1}\right)$, Caso B $\left(I_{2}>\right.$ $\left.I_{3}>I_{1}\right)$, Caso $\mathrm{C}\left(I_{1}>I_{3}>I_{2}\right)$, y Caso $\mathrm{D}\left(I_{3}<I_{2}, I_{1}\right)$. Por otro lado la relación $I_{2} / I_{3}, I_{1} /$ $I_{3}$, condiciona la respuesta dinámica de los sistemas dados por estos casos. 
d. Si consideramos el Caso A y el Caso D, si tomamos en consideración un determinado caso base de configuración de momentos de inercia, es posible apreciar que si partimos de una solución estable para $k_{31}=0$, aumentos de signo positivo del parámetro $k_{31}$ resultan en un alejamiento de los límites de estabilidad, respecto de la posición para $k_{31}=0$, mientras que valores negativos los disminuyen. Por otro lado un alejamiento del valor de $I_{3}$ al respecto de $I_{2}, I_{1}$, mejora la estabilidad del sistema, y a la inversa, su aproximación tiene como límite a la inestabilidad dinámica, independientemente del valor que tome $k_{31}$.

e. Al respecto de parámetros de desempeño, los ensayos realizados muestran que para $I_{3}$ $>I_{2}, I_{1}$, el parámetro $k$ (relación entre la velocidad de giro de las palas y la velocidad de caída) crece linealmente con el ángulo medio de paso de las palas, $\beta_{0}$, mientras que el coeficiente de resistencia aerodinámica $c_{D M}$ tiene un valor mínimo absoluto para determinado $\beta_{0}$.

f. Se puede observar en los casos simulados que el efecto de un aumento de la relación entre la pendiente de la curva de sustentación y el coeficiente de resistencia aerodinámica, $C_{L \alpha} / C_{D}$, cuando $k_{31}=0, k_{31}>0$ y $k_{31}<0$ es el de aumentar el tiempo hasta alcanzar la solución de equilibrio, a la vez que se acerca a los límites de estabilidad del sistema.

g. El Caso B muestra inestabilidad dinámica para $k_{31}=0$ que resulta de la configuración de los momentos de inercia. Resulta posible encontrar soluciones estables con $k_{31}>0$ que resultan inviables tecnológicamente hablando, puesto que $k_{31}$ es muy grande.

h. El Caso C muestra, idénticamente al Caso B, inestabilidad dinámica para $k_{31}=0$, que resulta de la configuración de los momentos de inercia. También en este caso es posible encontrar soluciones estables con $k_{31}>0$.

i. Si analizamos el efecto del parámetro $k_{21}$ para el Caso A es posible observar que para $k_{31}=0$ su efecto es el de disminuir el tiempo hasta alcanzar la solución de equilibrio mientras más grande sea su magnitud. Cuando el caso es que $k_{31}>0$ un aumento de $k_{21}$ 
conlleva un aumento de tiempo, y si $k_{31}<0$ una disminución. Este comportamiento se invierte cuando se trata del Caso D.

\subsection{ESTUDIOS EXPERIMENTALES}

Se ha estudiado experimentalmente el comportamiento dinámico de una configuración de decelerador aerodinámico sobre la base de dos tipos diferentes de ensayos que complementan aquellos realizados por Nadal Mora (2005). Por otro lado se estudian los ensayos realizados por Kellas (2007) que validan la capacidad de describir la actitud del pararrotor por parte del simulador numérico.

Cada uno de los ensayos ha permitido observar el comportamiento dinámico del pararrotor y determinar los parámetros que caracterizan el desempeño de la solución de equilibrio, como $k$ y $c_{D M}$, que son comunes a todos los ensayos realizados.

Se convalida a partir de los ensayos en el túnel aerodinámico vertical lo predicho por el simulador al respecto de la disminución de la relación de velocidades $k$ y del coeficiente de resistencia del modelo $c_{D M}$ al aumentar el ángulo de paso de las palas. Este hecho indica que los valores máximos de $c_{D M}$ se alcanzan a ángulos mínimos de $\beta_{1} \mathrm{y} \beta_{2}$.

Se confirma a partir de los ensayos realizados que es posible obtener soluciones estables bajo la condición $I_{3}>I_{2}, I_{1}$. Las configuraciones ensayadas, donde $I_{2}>I_{3}, I_{1}$, fueron inestables. Las simulaciones numéricas arrojan idéntica respuesta al respecto de la estabilidad del sistema para las condiciones medidas en los ensayos.

El comportamiento del ángulo de nutación es congruente entre ensayos y validan los resultados del simulador numérico. Se comprueba de los ensayos realizados que el ángulo de nutación aumenta al aumentar los valores absolutos de $\delta_{\beta}$ y $k_{31}$. Este efecto se verifica en las simulaciones numéricas. También es posible observar que para $\delta_{\beta}=0$ el ángulo de nutación de la solución de equilibrio es aproximadamente cero, y que un aumento de $\delta_{\beta}$ implica la 
aparición de soluciones de equilibrio con ángulos de nutación muy pequeños, denominados solución Tipo I en el Capítulo 3.

A partir del estudio de ensayos experimentales de referencia, y su comparación con su simulación numérica, se convalida la capacidad de la herramienta de simulación para describir la respuesta dinámica del sistema, desempeño y actitud. Por otro lado también se puede concluir a partir de estos estudios que es posible pensar configuraciones de pararrotor con grandes ángulos de ataque donde el flujo se encuentre totalmente desprendido de la superficie de las alas como sucede en las determinaciones de Heyson y Boswinke (1955), que son confirmadas por los ensayos realizados por Crimi (1988).

\subsection{CONCLUSIONES GENERALES}

Se puede concluir a partir de la observación de las simulaciones anteriores que para $I_{3}<I_{2}<$ $I_{1}$, es posible obtener 3 tipos de soluciones. La denominada Tipo I, II, inestabilidad dinámica.

El aumento de $k_{31}$ tiene dos efectos posibles de acuerdo a su magnitud y a la configuración geométrica y aerodinámica del pararrotor: uno de los efectos es el aumento del tiempo de respuesta al respecto de un caso idéntico con $k_{31}=0$, y el otro es el cambio de solución a la Tipo II. En cualquier caso un aumento significativo de $k_{31}$ tiende a disminuir la sensibilidad del pararrotor a factores desestabilizantes y presenta un efecto estabilizante. En general se demuestra a partir de las simulaciones realizadas que $k_{31}$ aporta a la estabilidad del sistema mientras mayor sea este parámetro (a pesar que su introducción promueva la aparición de soluciones de equilibrio tipo cónicas).

La conclusión general del presente capítulo es que a través de la variación de $I_{3}, I_{2}$ e $I_{1}, k_{21}$, $k_{31}, C_{L \alpha}, C_{D}, \beta_{1}$ y $\beta_{2}$ es posible controlar el comportamiento dinámico del pararrotor. En particular el tiempo de respuesta del sistema a una perturbación y la solución de equilibrio que determina la actitud del pararrotor son dependientes de estos parámetros. Sería posible por tanto controlar y guiar este tipo de dispositivos por medio de la manipulación de los parámetros mencionados. 
En los estudios analíticos se presentan dos parámetros asociados a la estabilidad de los sistemas, $N_{e}{ }^{\prime}$ y $k_{e}$, que resultan de la linealización del sistema y de la eliminación de aquellas magnitudes de menor orden en su expresión analítica. Se comprueba en las simulaciones realizadas que las regiones definidas por estos parámetros son útiles para describir las condiciones de estabilidad del sistema para el sistema que se simula numéricamente.

En los estudios analíticos se establecieron distintos casos de estabilidad en función de la relación de momentos de inercia. En las simulaciones numéricas se verifica que estos casos responden efectivamente de manera diferente frente a la variación de los parámetros analizados, y por tanto validan la utilidad de esta clasificación para la descripción de la estabilidad del sistema.

\subsubsection{Desempeño como desacelerador}

A partir de mediciones experimentales de la velocidad de caída $U_{\infty}$ y la velocidad de rotación $\omega_{3}$ es posible determinar y ajustar los valores de los coeficientes aerodinámicos $C_{L \alpha}$ y $C_{D}$ de manera que el error de los valores de las soluciones de equilibrio simuladas y aquellos de los casos ensayados se mantengan dentro de márgenes tolerables para la simulación. Vale decir que la determinación de los valores de $C_{L \alpha} \mathrm{y} C_{D}$ en función de parámetros que las afectan es necesario para que el equilibrio entre velocidad de rotación sobre el denominado eje 3 del pararrotor y la velocidad de descenso sobre el eje inercial $\mathrm{Z}$ simulado reproduzca la realidad física con un error tolerable.

Los coeficientes aerodinámicos $C_{L \alpha} \mathrm{y} C_{D}$ que surgen de los ensayos en el túnel vertical arrojan un promedio de $C_{L \alpha}=1,41$ y $C_{D}=0,146$ para un ajuste dado en el simulador numérico como se menciona en el Capítulo 4. Este par específico de valores resultan coherentes con aquellos calculados analíticamente o ensayados en trabajos anteriores como el de Hoerner y Borst (1975), y Pelletier y Mueller (2000). Estos hechos convalidan a la simulación numérica al respecto de su capacidad de reproducir el desempeño del pararrotor en términos de $U_{\infty} \mathrm{y} \omega_{3}$. 
Los parámetros $k$ y $c_{D M}$ en general dan cuenta del desempeño aerodinámico del pararrotor, estando constitutivamente vinculadas a las velocidades $U_{\infty} \mathrm{y} \omega_{3}$.

El parámetro $c_{D M}$ permite medir a partir de un coeficiente adimensional, el desempeño general del pararrotor como desacelerador.

Los ensayos en túnel vertical indican que el parámetro $k$ aumenta linealmente con el aumento de $\beta_{0}$, con $\beta_{1}=\beta_{2}$, tal cual se indica en el Capítulo 4. Las simulaciones numéricas de la dinámica del pararrotor muestran idéntico comportamiento, como se indica en el Capítulo 3. Por otro lado se observa de los ensayos experimentales que $k$ disminuye al aumentar la diferencia de paso de las palas, $\delta_{\beta}$.

Las simulaciones numéricas de la dinámica del pararrotor indican la existencia de un mínimo absoluto en la curva de $c_{D M}$ en función de $\beta_{0}$, como se indica en el Capítulo 3, para valores positivos de $\beta_{0}$. Los ensayos realizados en el túnel vertical ratifican este comportamiento por cuanto muestran que la mejor curva de interpolación entre los datos medidos es cuadrática. Resulta recomendable el estudio experimental de la variación de estos mínimos absolutos en función de parámetros de configuración geométrica y bajo qué condiciones es posible alcanzarlos.

\subsubsection{Comportamiento dinámico y estabilidad del pararrotor}

De acuerdo al modelo analítico propuesto, la estabilidad del pararrotor se encuentra definida en mayor medida por la relación de los momentos de inercia del dispositivo.

Las simulaciones numéricas muestran, la existencia de tres tipos de respuestas dinámicas del pararrotor:

- Solución Tipo I. Este tipo de solución agrupa a todas aquellas en donde el ángulo de nutación $\theta_{e}$ es nulo o muy pequeño. 
- Solución Tipo II. Este tipo de solución agrupa a todas aquellas en donde el ángulo de nutación $\theta_{e}$ tiene un valor medio que no es pequeño que permanece constante en el tiempo. Suelen llamarse también soluciones tipo cónicas.

\section{- Inestabilidad dinámica}

El modelo analítico linealizado no predice la existencia de soluciones Tipo II (ni podría hacerlo bien pues ocurren a ángulos de nutación grandes, hipótesis asumida en el modelo referido). De los casos simulados se observa que para determinados valores de coeficientes aerodinámicos de las palas, el modelo analítico y la simulación numérica son congruentes en la estimación de la solución de equilibrio cuando esta es de la denominada Tipo I.

En el Capítulo 3 se presenta una serie de simulaciones realizadas con el objeto de explorar distintas zonas del gráfico $N_{e}$, $k_{e}$, para el caso que $I_{3}>I_{2} \geq I_{1}$ y $k_{31}=0$. El modelo analítico presentado por Nadal Mora (2005), y el desarrollado en este documento, prevén una zona de estabilidad definida por $N_{e}{ }^{\prime}>1$. Las simulaciones numéricas muestran el comportamiento del pararrotor incluyendo efectos no lineales y efectos de órdenes de magnitud menores no contemplados en el modelo analítico. El resultado en términos de regiones en el gráfico $N_{e}$, $k_{e}$ es la existencia de tres tipos de soluciones asociadas a regiones bien definidas, que incluye las denominadas Tipo II. Se pudo observar que para casos donde $k_{e}>20$ existe un límite entre soluciones Tipo I y Tipo II dado por un valor aproximado de $N_{e}{ }^{\prime}=0,69$; y entre soluciones Tipo II y soluciones inestables dado por un valor aproximado de $N_{e}{ }^{\prime}=0,90$. Para valores de $k_{e}$ menores a 20 los límites se van desplazando hacia valores mayores de $N_{e}$ ' conforme se disminuye $k_{e}$. Esta disminución se observa asintótica para el límite entre soluciones estables e inestables para valores de $k_{e}=0$.

Estos resultados convalidan la existencia de regiones en el gráfico $N_{e}{ }^{\prime}, k_{e}$ de estabilidad predicho por el sistema analítico, evidenciando la utilidad de la utilización de los parámetros $N_{e}{ }^{\prime}, k_{e}$ para la descripción del comportamiento dinámico del sistema y su estabilidad.

Futuras investigaciones podrían abordar el estudio del efecto de distintos parámetros de configuración al respecto de las regiones definidas. 
El efecto del desplazamiento del centro de sustentación respecto del centro de masas, $k_{31}$, puede resumirse como:

- aumenta el tiempo de establecimiento de la solución de equilibrio,

- introduce soluciones Tipo II,

- en el caso que $k_{31}>0$, al respecto de casos donde $k_{31}=0$, su efecto resulta en un alejamiento de los límites de estabilidad,

- en el caso que $k_{31}>0$, al respecto de casos donde $k_{31}=0$, su efecto resulta en un acercamiento a los límites de estabilidad del sistema.

El efecto del desplazamiento del centro de presiones en dirección de la cuerda de la pala, $k_{21}$, al respecto del comportamiento dinámico del pararrotor, depende de la relación de momentos de inercia que se presente. Si tomamos el caso que $I_{3}>I_{2} \geq I_{1}$ estos efectos pueden resumirse como:

- un aumento de $k_{21}$ se corresponde con una diminución del tiempo de establecimiento de la solución de equilibrio, para $k_{31}<0$ y $k_{31}=0$.

- se presenta un mínimo absoluto en términos de tiempo de establecimiento de la solución de equilibrio para valores positivos de $k_{21}$, para el caso que $k_{31}>0$.

Si tomamos el caso que $I_{1} \geq I_{2}>I_{3}$ estos efectos pueden resumirse como:

- un aumento de $k_{21}$ se corresponde con un aumento del tiempo de establecimiento de la solución de equilibrio, para $k_{31}>0$ y $k_{31}=0$.

- un aumento de $k_{21}$ se corresponde con una disminución del tiempo de establecimiento de la solución de equilibrio, para $k_{31}<0$.

Con relación al parámetro $C_{L \alpha} / C_{D}$, este resulta condicionante de la respuesta dinámica del pararrotor. Los desarrollos analíticos asociados a su determinación como placas planas de 
bajo alargamiento (Anexo 1) indican que esta relación aumenta al aumentar el ángulo de ataque de las palas. El comportamiento dinámico asociado a este aumento es el acercamiento del comportamiento dinámico a los límites de estabilidad.

\subsection{FUTURAS LÍNEAS DE INVESTIGACIÓN}

Las futuras líneas de investigación asociadas al estudio del pararrotor tienen distintas vertientes, las cuales se explicitan a continuación.

Por un lado resulta de interés explorar a partir de las herramientas de simulación numérica generadas en el presente trabajo el efecto de los parámetros $k_{21}, C_{L \alpha} / C_{D}, k_{31}$ sobre los parámetros $N_{e}{ }^{\prime}, k_{e}$ en relación al comportamiento dinámico del sistema.

Por otro lado podrían establecerse estrategias de control y guiado del dispositivo a partir del modelo matemático desarrollado y otros nuevos modelos, incluyendo el estudio de acciones de control técnicamente practicables.

Al respecto de estudios experimentales, resulta aconsejable que a partir de ensayos en túnel vertical y/o ensayos en vuelo se verifiquen los distintos comportamientos dinámicos predichos y se midan los ángulos de nutación asociados, de manera de poder verificar los modelos numérico y analítico sobre la base de una serie extensa de ensayos.

Al respecto de la configuración geométrica del pararrotor, resulta de interés establecer el efecto del alargamiento de las palas y del diedro sobre la dinámica del sistema desde un punto experimental y a partir de simulaciones numéricas. En el caso particular del diedro, su existencia implica un impacto en el parámetro $k_{31}$ que puede ser de singular interés para el establecimiento de acciones de control del pararrotor.

\subsection{APORTES ORIGINALES DE LA PRESENTE TESIS}

Los aportes originales que se presentan en el presente trabajo pueden mencionarse como: 
- el desarrollo de un modelo analítico del sistema dinámico contemplando el efecto del desplazamiento del plano que contiene a las palas al respecto del centro de masas de manera de predecir su efecto,

- la descripción de la respuesta dinámica y solución de equilibrio del sistema a partir de parámetros adimensionales que sintetizan el efecto de un gran número de parámetros de configuración del pararrotor,

- el desarrollo de una herramienta de simulación numérica genérica que permite verificar la dinámica del sistema conjuntamente con su actitud espacial, la cual posibilita el estudio de múltiples configuraciones y posteriores estudios; y su validación por ensayos realizados para la presente tesis y otros realizados por otros autores,

- la clasificación de los distintos tipos de respuesta dinámica de los pararrotores en función del ángulo de nutación de equilibrio, determinando tipologías de solución de equilibrio,

- la determinación del efecto de diversos parámetros de la configuración, como los momentos de inercia, la distancia en dirección de la cuerda del centro aerodinámico de presiones y el centro de sustentación, $k_{21}$, la relación de la pendiente de sustentación de las palas y el coeficiente de resistencia, $C_{L \alpha} / C_{D}$, la distancia del centro de sustentación al centro de masas, $k_{31}$, sobre la dinámica del sistema y las soluciones de equilibrio, a partir de la simulación numérica de casos específicos,

- el estudio del comportamiento de parámetros de desempeño a partir de la simulación numérica y los ensayos experimentales que permitirían la optimización de modelos con funciones específicas y/o la gestión del desempeño como desacelerador,

- la identificación de posibles acciones de control para el guiado y control de dispositivos. 


\section{ANEXO 1}

\section{ESTUDIO ACERCA DE LOS COEFICIENTES AERODINÁMICOS DE PLACAS DE BAJO ALARGAMIENTO}

\subsection{INTRODUCCIÓN}

El pararrotor es un dispositivo desacelerador aerodinámico basado en la autorrotación de alas giratorias. La utilización práctica de este dispositivo que despierta mayor interés suele traer aparejado el uso de alas de bajo alargamiento, menor a uno, asimilables en general a placas planas. Esta configuración geométrica surge a partir de la necesidad de contar con un dispositivo desacelerador, de pequeñas dimensiones, económico, liviano y simple.

A lo largo de los estudios que se realizaron en el trabajo que acompaña el presente apartado se ha supuesto la utilización de alas que cuentan con coeficientes $C_{D}$ y $C_{L \alpha}$ constantes. Nadal Mora (2005) considerara modelos con coeficientes de resistencia constantes y variables. Existen efectos asociados a la tridimensionalidad del movimiento del aire alrededor de las alas que resultan en coeficientes aerodinámicos variables con el ángulo de ataque y corrimientos del centro aerodinámico de presiones al respecto de su ubicación en alas de gran alargamiento.

\subsection{COEFICIENTES AERODINÁMICOS}

\subsubsection{Modelo del coeficiente de sustentación}

Se puede observar que para placas planas de pequeño alargamiento, la pendiente de la curva de sustentación aumenta con el ángulo de ataque hasta el ángulo de perdida en lugar de responder linealmente como el caso de grandes relaciones de alargamiento (ver Figura A1-2). 
Hoerner presenta una metodología para la estimación de los coeficientes aerodinámicos para relaciones de alargamiento menores a 1 , que cuentan con comprobada precisión y que han sido validadas experimentalmente por otros autores.

Bajo este enfoque, para la estimación del coeficiente de sustentación se distinguen dos componentes de la fuerza de sustentación: una que varía linealmente con el ángulo de ataque y otra cuya variación es no lineal.

La componente lineal, consecuencia de la circulación del aire, para relaciones de alargamiento menores a 1, tiene la siguiente expresión:

$$
C_{L \alpha \text { lineal }}=\frac{1}{2} \pi \Lambda
$$

donde $\Lambda$ es el alargamiento de las alas.

Esta expresión se corresponde con los resultados de aplicar a una placa de longitud infinita el teorema de Kutta-Yukovski.

La componente no lineal se la asocia al efecto tridimensional de los bordes laterales de las placas. Su formulación tiene base empírica elaborada por Hoerner (1975). Para este modelo el incremento del coeficiente de sustentación será función de la relación de aspecto de las alas, los bordes laterales y la componente normal de la velocidad incidente. La expresión de la componente no lineal es entonces:

$$
C_{L \alpha \text { no lineal }}=k^{\prime} \sin ^{2} \alpha \cos \alpha .
$$

El coeficiente $k$ ' dependerá del alargamiento de la placa y el tipo de borde. Para placas planas de bordes afilados y alargamiento de 0,6 , se comprueba experimentalmente que $k^{\prime}=3,5$, que corresponde al valor máximo de este coeficiente. En la Figura A1-1 se presentan los valores de $k^{\prime}$ con respecto al coeficiente de alargamiento, que resultan congruentes a los valores experimentales hallados por Pelletier (2000), Kuchemann (1952), y otros autores. 


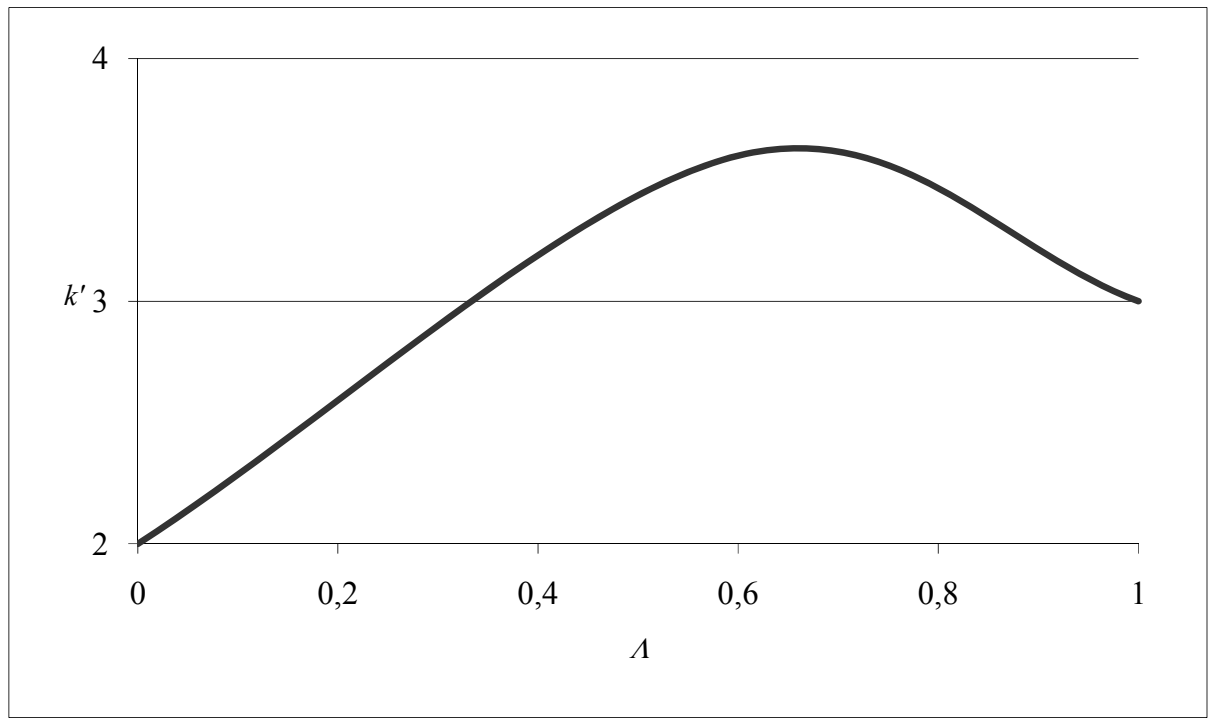

Figura A1- 1 Curva empírica de $k^{\prime}$ en función de la relación de alargamiento de una placa plana rectangular de esquinas no redondeadas ni achaflanadas. Hoemer (1975).

La formulación general será entonces, para $\Lambda<1$ :

$C_{L \alpha}=\frac{1}{2} \pi \Lambda+k^{\prime} \sin ^{2} \alpha \cos \alpha$ 


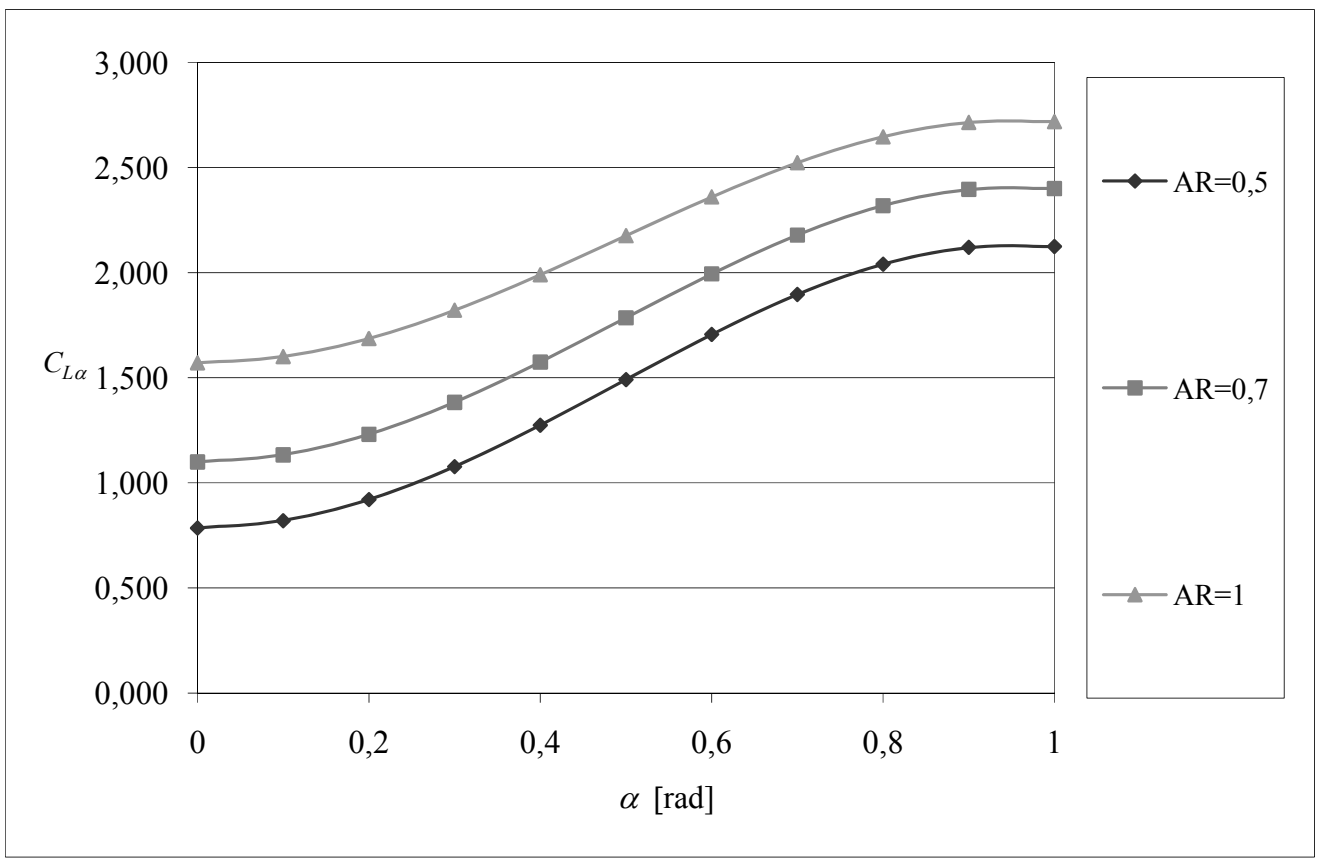

Figura A1 - 1 Curva de $C_{L \alpha}$ contemplando la componente lineal y la componente no lineal.

Otros autores como Pelletier (2000) y Leving (1992) han elaborado otros modelos semiempíricos para predecir el comportamiento del coeficiente de sustentación en alas de pequeño alargamiento. Tanto los ensayos de laboratorio como los modelos desarrollados son congruentes cualitativamente y cuantitativamente con aquel que se presenta en este apartado, que introduce Hoerner (1975).

Se puede observar que los pequeños alargamientos de las palas implican la introducción de comportamientos aerodinámicos tridimensionales importantes cuyo efecto resulta en un aumento de las fuerzas de sustentación.

\subsubsection{Modelo del coeficiente de resistencia}

El modelo que se adopta para la descripción de la resistencia en alas de pequeño alargamiento resulta congruente para autores como Kuchemann (1952) y Hoerner (1975). Para la evaluación de la resistencia de cada ala del pararrotor se distinguieron las distintas 
componentes que aportan a la resistencia: resistencia de piel, resistencia inducida y resistencia debida a los vórtices de extremo de pala. Su expresión resulta:

resistencia de fricción y de forma: $C_{D 0}$,

donde según datos experimentales para placas planas la resistencia por roce en la superficie es:

$C_{D 0}=0,06$;

resistencia inducida: $\quad \frac{C_{\text {La lineal }}^{2}}{\pi \Lambda}$;

resistencia inducida debida a los vórtices que se generan en la pala:

$$
C_{\text {La no lineal }} \alpha
$$

Por tanto:

$C_{D}=C_{D 0}+\frac{C_{\text {Llineal }}^{2}}{\pi \Lambda}+C_{\text {Lnolineal }} \alpha$

Para relaciones de alargamiento menores a 1 esta expresión resulta:

$C_{D}=C_{D 0}+\frac{1}{4} \pi \Lambda \alpha^{2}+k^{\prime} \sin ^{2} \alpha \cos \alpha \alpha^{2}$

Por otro lado los datos experimentales de Pelletier son congruentes con este modelo.

\subsubsection{Variación de $C_{L \alpha} / C_{D}$ con el ángulo de ataque}

Si se dividen las expresiones (A1-3) y (A1-5) es posible obtener una curva de variación de $C_{L \alpha} / C_{D}$ en función de ángulo de ataque como la que se muestra en la Figura A1-3. De 
acuerdo a las expresiones de referencia el parámetro $C_{L \alpha} / C_{D}$ es decreciente con el ángulo de ataque.

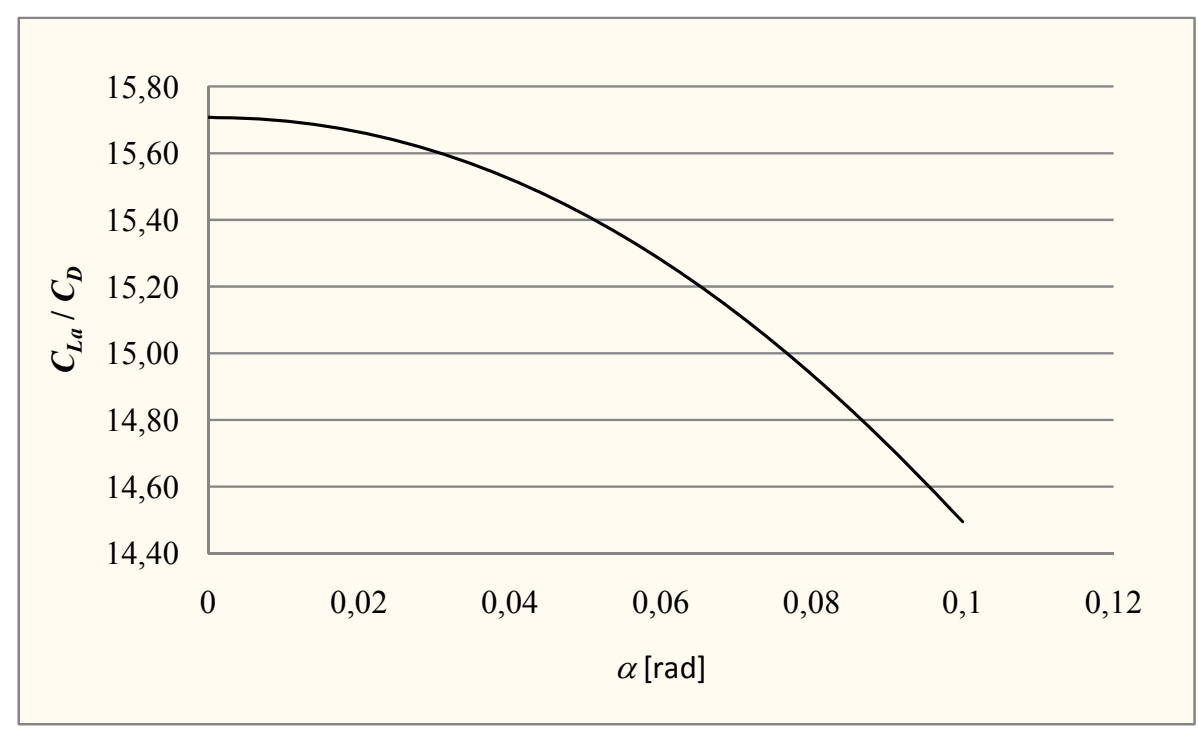

Figura A1 - $2 C_{L \alpha} / C_{D}$ en función del ángulo de ataque $\alpha$ para una pala de características típicas.

\subsection{CENTRO DE PRESIONES}

El autor Kurcheman (1952) elaboró un modelo de distribución de presiones de la componente lineal de la sustentación para alas de bajo alargamiento que se ajusta a la información experimental disponible. Este modelo permite la determinación del centro de presiones en función. La formulación que se propone para la distribución de carga en dirección de la cuerda es:

$C_{P}(x)=-\frac{\sin \pi n}{\pi n} C_{L \alpha}\left(\frac{1-x}{x}\right)^{n}$

donde $n$ es un coeficiente asociado al alargamiento y $x$ es la posición a lo largo de la cuerda. 
Se puede observar que para relaciones de alargamiento muy grandes $n$ tiende a $1 / 2$ y (A1-6) se corresponde con la distribución de presiones para una placa plana sin considerar efectos tridimensionales, aplicando la ley de Biot-Savart a la distribución de Birnbaum.

También se puede observar que cuando la relación de aspecto tiende a 0 el valor de $n$ deber tender a 1, para una distribución concentrada en el borde de ataque, congruente con la denominada solución de R. T. Jones. Por tanto la distribución (AI-6) incluye una familia de funciones de carga que incluye los dos casos extremos.

Para el caso que $\Lambda<1$, se encuentra que el comportamiento del parámetro $n$ responde a la siguiente expresión:

$$
n=1-\sqrt{\frac{1}{8}}
$$

La posición del centro aerodinámico de presiones asociado a este modelo de distribución de presiones resulta de la expresión que sigue:

$$
x_{p \%}=\frac{1-n}{2} \cdot 100 \%
$$

Se puede ver que para una relación de alargamiento de 0,65 , el centro de presiones se ubica a un $14 \%$ de la cuerda desde el borde de ataque.

\subsection{CONCLUSIONES}

A partir de lo expuesto en este apartado es posible decir que la disminución del alargamiento de las palas del pararrotor implica:

- $C_{L \alpha}$ disminuye al bajar $\Lambda$ para igual $\alpha$,

- $C_{D}$ disminuye al bajar $\Lambda$ para igual $\alpha$,

- $C_{L \alpha} / C_{D}$ disminuye con $\alpha$, 
- el centro de presiones se mueve hacia el borde de ataque, por lo tanto aumenta el valor del parámetro $k_{21}$, al bajar $\Lambda$ para igual $\alpha$. 


\section{ANEXO 2}

\section{RESULTADOS EXPERIMENTALES}

\section{$2.1 \quad$ INTRODUCCIÓN}

Para el estudio experimental del dispositivo desacelerador basado en la autorrotación de alas giratorias, pararrotor, se prepararon una serie de ensayos sobre la base modelos físicos del dispositivo.

En el presente apartado se exponen los registros de los ensayos realizados, a saber:

c) ensayos en túnel aerodinámico vertical sobre un modelo en vuelo libre

d) ensayos en túnel aerodinámico sobre un modelo que rota sobre un rodamiento basculante.

\subsection{ENSAYOS EN TÚNEL AERODINÁMICO VERTICAL SOBRE UN MODELO EN VUELO LIBRE}

Tabla A2 - 1 Descripción de los modelos ensayados.

\begin{tabular}{|c|c|c|c|c|c|c|}
\hline Denominación & $\begin{array}{c}\text { Peso } \\
{[\mathrm{N}]}\end{array}$ & $\begin{array}{c}h \\
{[\mathrm{~mm}]}\end{array}$ & $\begin{array}{c}e \\
{[\mathrm{~mm}]}\end{array}$ & $\begin{array}{c}I_{1} \\
{\left[\mathrm{~kg} \mathrm{~m}^{2}\right]}\end{array}$ & $\begin{array}{c}I_{2} \\
{\left[\mathrm{~kg} \mathrm{~m}^{2}\right]}\end{array}$ & $\begin{array}{c}I_{3} \\
{\left[\mathrm{~kg} \mathrm{~m}^{2}\right]}\end{array}$ \\
\hline$C 05 P 01$ & 0,15 & 26 & 0,5 & $3,5 \cdot 10^{-6}$ & $8,9 \cdot 10^{-6}$ & $11,6 \cdot 10^{-6}$ \\
\hline$C 05 P 02$ & 0,20 & 26 & 0,8 & $5,4 \cdot 10^{-6}$ & $21,2 \cdot 10^{-6}$ & $25,9 \cdot 10^{-6}$ \\
\hline$C 05 P 03$ & 0,11 & 26 & 0,3 & $1,9 \cdot 10^{-6}$ & $5,8 \cdot 10^{-6}$ & $6,9 \cdot 10^{-6}$ \\
\hline
\end{tabular}




\begin{tabular}{|c|c|c|c|c|c|c|}
\hline$C 06 P 02$ & 0,28 & 56 & 0,8 & $9,5 \cdot 10^{-6}$ & $25,3 \cdot 10^{-6}$ & $27,1 \cdot 10^{-6}$ \\
\hline$C 06 P 03$ & 0,19 & 56 & 0,3 & $5,9 \cdot 10^{-6}$ & $9,8 \cdot 10^{-6}$ & $8,1 \cdot 10^{-6}$ \\
\hline$C 05 P 02 M 01$ & $0,22 *$ & 26 & 0,8 & $10,1 \cdot 10^{-6}$ & $22,5 \cdot 10^{-6}$ & $31,8 \cdot 10^{-6}$ \\
\hline
\end{tabular}

Tabla A2 - 2 Descripción del instrumental utilizado en los ensayos de túnel vertical.

\begin{tabular}{|c|c|c|c|c|}
\hline Instrumento & Marca & Modelo/Tipo & Rango & Resolución \\
\hline $\begin{array}{c}\text { Lámpara } \\
\text { estroboscópica }\end{array}$ & - & - & - & $50 \mathrm{rpm}$ \\
\hline Tacómetro Láser & Lutron & DT-2234BL & 10 a 99999 rpm & $\begin{array}{c}0,1 \mathrm{rpm}(<1000 \mathrm{rpm}) \\
1 \mathrm{rpm}(>1000 \mathrm{rpm})\end{array}$ \\
\hline Tubo Pitot & Estándar & $\begin{array}{c}\text { NPL } \\
\text { Iram } 19004\end{array}$ & - & - \\
\hline $\begin{array}{c}\text { Micromanómetro } \\
\text { diferencial }\end{array}$ & R-Fuess & & 0 a $160 \mathrm{mmCA}$ & $0,05 \mathrm{mmCA}$ \\
\hline Barómetro & Druck & DPI740 & 0 a $400 \mathrm{~Pa}$ & $0,1 \mathrm{~Pa}$ \\
\hline $\begin{array}{c}\text { Termómetro de } \\
\text { bulbo seco y bulbo } \\
\text { húmedo }\end{array}$ & & & $\begin{array}{c}\mathrm{BH}-25^{\circ} \mathrm{Ca} \\
35^{\circ} \mathrm{C} \\
\text { BS } 0^{\circ} \mathrm{C} \mathrm{a} 100^{\circ} \mathrm{C} \\
\end{array}$ & $0,1^{\circ} \mathrm{C}$ \\
\hline $\begin{array}{c}\text { Balanza } \\
\text { electrónica }\end{array}$ & - & - & $\begin{array}{c}0 \text { a } 5000 \\
\text { gramos }\end{array}$ & 10 gramos \\
\hline Goniómetro & - & - & 0 a 90 grados & 1 grado \\
\hline $\begin{array}{l}\text { Cámara } \\
\text { fotográfica sobre } \\
\text { registro de fondo }\end{array}$ & Sony & DSC-W110 & - & - \\
\hline
\end{tabular}

Tabla A2 - 3 Ensayos realizados en túnel vertical

\begin{tabular}{|c|c|c|c|c|c|}
\hline Configuración & $\begin{array}{c}\boldsymbol{\beta}_{\mathbf{1}} \\
{\left[{ }^{\mathbf{0}}\right]}\end{array}$ & $\begin{array}{c}\boldsymbol{\beta}_{2} \\
{\left[{ }^{\circ}\right]}\end{array}$ & $\begin{array}{c}\boldsymbol{\delta} \boldsymbol{\beta} \\
{\left[{ }^{\mathbf{0}}\right]}\end{array}$ & $\begin{array}{c}\boldsymbol{V}_{\boldsymbol{v}} \\
{[\mathbf{m} / \mathbf{s}]}\end{array}$ & $\begin{array}{c}\boldsymbol{\omega}_{3} \\
{[\mathbf{r a d} / \mathbf{s}]}\end{array}$ \\
\hline$C 05 P 01$ & -12 & 10 & -11 & 5,4 & 216 \\
\hline
\end{tabular}




\begin{tabular}{|c|c|c|c|c|c|}
\hline Configuración & $\begin{array}{l}\beta_{1} \\
{\left[{ }^{\circ}\right]}\end{array}$ & $\begin{array}{l}\beta_{2} \\
{\left[^{0}\right]}\end{array}$ & $\begin{array}{l}\delta \beta \\
{\left[{ }^{0}\right]}\end{array}$ & $\begin{array}{c}V_{v} \\
{[\mathrm{~m} / \mathrm{s}]}\end{array}$ & $\begin{array}{c}\omega_{3} \\
{[\mathrm{rad} / \mathrm{s}]}\end{array}$ \\
\hline C05P02 & -12 & 10 & -11 & 6,2 & 237 \\
\hline C05P02 & -12 & 8 & -10 & 6,5 & 293 \\
\hline C05P02 & -10 & 8 & -9 & 6,2 & 253 \\
\hline C05P02 & -10 & 6 & -8 & 6,3 & 308 \\
\hline$C 05 P 02$ & -10 & 4 & -7 & 6,3 & 344 \\
\hline$C 05 P 02$ & -10 & -10 & 0 & 6,2 & 237 \\
\hline C05P02 & -8 & 10 & -9 & 6,4 & 229 \\
\hline C05P02 & -8 & 6 & -7 & 6,0 & 263 \\
\hline C05P02 & -8 & 4 & -6 & 6,1 & 290 \\
\hline C05P02 & -8 & 2 & -5 & 6,2 & 319 \\
\hline C05P02 & -8 & 0 & -4 & 6,2 & 453 \\
\hline C05P02 & -8 & -4 & -2 & 6,3 & 505 \\
\hline C05P02 & -8 & -8 & 0 & 6,6 & 551 \\
\hline$C 05 P 02$ & -8 & -8 & 0 & 7,8 & 429 \\
\hline$C 05 P 02$ & -6 & 4 & -5 & 6,2 & 241 \\
\hline$C 05 P 02$ & -6 & 2 & -4 & 6,2 & 287 \\
\hline$C 05 P 02$ & -6 & -6 & 0 & 6,4 & 468 \\
\hline C05P02 & -6 & -8 & 1 & 7,3 & 516 \\
\hline$C 05 P 02$ & -4 & -4 & 0 & 6,3 & 428 \\
\hline C05P02 & -4 & -6 & 1 & 6,6 & 444 \\
\hline C05P02 & -4 & -8 & 2 & 6,8 & 474 \\
\hline$C 05 P 02$ & -2 & -2 & 0 & 6,2 & 400 \\
\hline C05P02M01 & -8 & -8 & 0 & 7,6 & 530 \\
\hline C05P02M01 & -6 & -6 & 0 & 7,4 & 480 \\
\hline C05P02M01 & -4 & -4 & 0 & 7,1 & 404 \\
\hline$C 05 P 02 M 01$ & -2 & -2 & 0 & 6,9 & 317 \\
\hline$C 05 P 03$ & -12 & 10 & -11 & 6,3 & 281 \\
\hline$C 05 P 03$ & -10 & 8 & -9 & 6,1 & 298 \\
\hline
\end{tabular}




\begin{tabular}{|c|c|c|c|c|c|}
\hline Configuración & $\begin{array}{l}\beta_{1} \\
{\left[{ }^{\circ}\right]}\end{array}$ & $\begin{array}{l}\beta_{2} \\
{\left[{ }^{0}\right]}\end{array}$ & $\begin{array}{l}\delta \boldsymbol{\beta} \\
{\left[{ }^{0}\right]}\end{array}$ & $\begin{array}{c}V_{v} \\
{[\mathrm{~m} / \mathrm{s}]}\end{array}$ & $\begin{array}{c}\omega_{3} \\
{[\mathrm{rad} / \mathrm{s}]}\end{array}$ \\
\hline$C 05 P 03$ & -6 & 0 & -3 & 6,0 & 348 \\
\hline$C 05 P 03$ & -6 & -6 & 0 & 6,5 & 477 \\
\hline$C 05 P 03$ & -4 & -4 & 0 & 6,2 & 363 \\
\hline$C 05 P 03$ & -4 & -6 & 1 & 6,3 & 439 \\
\hline$C 06 P 02$ & -8 & -8 & 0 & 9,2 & 507 \\
\hline С06P02 & -6 & -6 & 0 & 8,9 & 651 \\
\hline С06P02 & -4 & -4 & 0 & 8,9 & 578 \\
\hline С06P02 & -2 & -2 & 0 & 8,5 & 479 \\
\hline$C 06 P 03$ & -6 & -6 & 0 & 7,3 & 536 \\
\hline$C 06 P 03$ & -4 & -4 & 0 & 7,3 & 476 \\
\hline С06P03 & -2 & -2 & 0 & 7,0 & 395 \\
\hline
\end{tabular}

\subsection{ENSAYOS EN TÚNEL AERODINÁMICO SOBRE UN MODELO QUE ROTA SOBRE UN RODAMIENTO BASCULANTE}

Tabla A2 - 4 Parámetros distintivos de los modelos del pararrotor ensayados.

\begin{tabular}{|c|c|c|c|c|}
\hline$k_{31}$ & $\begin{array}{c}\text { Peso } \\
{[N]}\end{array}$ & $\begin{array}{c}I_{1} \\
{\left[\mathrm{~kg} \mathrm{~m}^{2}\right]} \\
\end{array}$ & $\begin{array}{c}I_{2} \\
{\left[\mathrm{~kg} \mathrm{~m}^{2}\right]}\end{array}$ & $\begin{array}{c}I_{3} \\
{\left[\mathrm{~kg} \mathrm{~m}^{2}\right]}\end{array}$ \\
\hline 0 & 3,5 & $4,61 \cdot 10^{-4}$ & $1,31 \cdot 10^{-3}$ & $1,48 \cdot 10^{-3}$ \\
\hline 0,18 & 4,2 & $5,30 \cdot 10^{-4}$ & $1,56 \cdot 10^{-3}$ & $1,66 \cdot 10^{-3}$ \\
\hline$-0,18$ & 4,2 & $5,30 \cdot 10^{-4}$ & $1,56 \cdot 10^{-3}$ & $1,66 \cdot 10^{-3}$ \\
\hline
\end{tabular}

Tabla A2 - 5 Valores medios de los parámetros medidos de distintas configuraciones de pararrotor. 


\begin{tabular}{|c|c|c|c|c|c|c|c|c|}
\hline $\boldsymbol{k}_{\mathbf{3 1}}$ & $\begin{array}{c}\boldsymbol{\beta}_{\mathbf{1}} \\
\text { [grados] }\end{array}$ & $\begin{array}{c}\boldsymbol{\beta}_{\mathbf{2}} \\
\text { [grados] }\end{array}$ & $\begin{array}{c}\boldsymbol{\delta}_{\boldsymbol{\beta}} \\
\text { [grados] }\end{array}$ & $\begin{array}{c}\boldsymbol{\varepsilon} \\
\text { [grados] }\end{array}$ & $\mathbf{1 0}^{-\mathbf{3}} \mathbf{R e}$ & $\boldsymbol{k}$ & $\boldsymbol{C}_{\boldsymbol{D M}}$ & $\begin{array}{c}\boldsymbol{\theta}_{\boldsymbol{e}} \\
\text { [grados] }\end{array}$ \\
\hline 0 & -4 & -4 & 0 & -8 & 250 & 0,27 & 3,22 & 0,18 \\
\hline 0 & -4 & -8 & 2 & -12 & 293 & 0,25 & 3,44 & 1,65 \\
\hline 0 & -4 & -12 & 4 & -16 & 286 & 0,27 & 3,44 & 2,06 \\
\hline$-0,18$ & -4 & -4 & 0 & -8 & 207 & 0,31 & 3,47 & 0,08 \\
\hline$-0,18$ & -4 & -8 & 2 & -12 & 262 & 0,30 & 3,32 & 4,75 \\
\hline$-0,18$ & -4 & -12 & 4 & -16 & 252 & 0,26 & 3,43 & 6,22 \\
\hline 0,18 & -4 & -4 & 0 & -8 & 296 & 0,26 & 3,55 & 0,51 \\
\hline 0,18 & -4 & -8 & 2 & -12 & 265 & 0,24 & 3,36 & 3,08 \\
\hline 0,18 & -4 & -12 & 4 & -16 & 300 & 0,26 & 3,19 & 6,37 \\
\hline
\end{tabular}

Tabla A2 - 6 Valores medios de los parámetros medidos de distintas configuraciones de pararrotor.

\begin{tabular}{|c|c|c|c|c|c|c|}
\hline $\boldsymbol{k}_{31}$ & $\begin{array}{c}\boldsymbol{\beta}_{1} \\
{[\text { grados] }}\end{array}$ & $\begin{array}{c}\boldsymbol{\beta}_{2} \\
{[\mathbf{g r a d o s}]}\end{array}$ & $\begin{array}{c}\boldsymbol{\delta}_{\boldsymbol{\beta}} \\
{[\mathbf{g r a d o s}]}\end{array}$ & $\begin{array}{c}\boldsymbol{U} \\
{[\mathbf{m} / \mathbf{s}]}\end{array}$ & $\begin{array}{c}\boldsymbol{\omega}_{3} \\
{[\mathbf{r a d} / \mathbf{s}]}\end{array}$ & $\mathbf{1 0}^{-3} \boldsymbol{R} \boldsymbol{2}$ \\
\hline-1 & -12 & -4 & -8 & 10 & 246 & 281 \\
\hline-1 & -12 & -4 & -8 & 10 & 262 & 299 \\
\hline-1 & -12 & -4 & -8 & 10 & 230 & 263 \\
\hline-1 & -12 & -4 & -8 & 12 & 266 & 304 \\
\hline-1 & -12 & -4 & -8 & 12 & 286 & 326 \\
\hline-1 & -12 & -4 & -8 & 12 & 307 & 351 \\
\hline-1 & -8 & -4 & -4 & 10 & 233 & 265 \\
\hline-1 & -8 & -4 & -4 & 10 & 224 & 255 \\
\hline-1 & -8 & -4 & -4 & 12 & 262 & 299 \\
\hline-1 & -8 & -4 & -4 & 12 & 272 & 310 \\
\hline-1 & -8 & -4 & -4 & 12 & 281 & 320 \\
\hline-1 & -4 & -4 & 0 & 10 & 186 & 212 \\
\hline-1 & -4 & -4 & 0 & 10 & 215 & 246 \\
\hline-1 & -4 & -4 & 0 & 12 & 253 & 288 \\
\hline
\end{tabular}




\begin{tabular}{|c|c|c|c|c|c|c|}
\hline$k_{31}$ & $\begin{array}{c}\beta_{1} \\
\text { [grados] }\end{array}$ & $\begin{array}{c}\beta_{2} \\
\text { [grados] }\end{array}$ & $\begin{array}{c}\delta_{\beta} \\
\text { [grados] }\end{array}$ & $\begin{array}{c}U \\
{[\mathbf{m} / \mathbf{s}]}\end{array}$ & $\begin{array}{c}\omega_{3} \\
{[\mathrm{rad} / \mathrm{s}]}\end{array}$ & $10^{-3} R e$ \\
\hline-1 & -4 & -4 & 0 & 12 & 220 & 251 \\
\hline-1 & -4 & -4 & 0 & 12 & 247 & 281 \\
\hline-1 & -2 & -4 & 2 & 10 & 130 & 149 \\
\hline-1 & -2 & -4 & 2 & 12 & 161 & 184 \\
\hline 0 & -12 & -4 & -8 & 10 & 290 & 331 \\
\hline 0 & -12 & -4 & -8 & 10 & 258 & 294 \\
\hline 0 & -12 & -4 & -8 & 10 & 259 & 295 \\
\hline 0 & -12 & -4 & -8 & 10 & 267 & 305 \\
\hline 0 & -12 & -4 & -8 & 10 & 261 & 298 \\
\hline 0 & -12 & -4 & -8 & 12 & 298 & 339 \\
\hline 0 & -8 & -4 & -4 & 10 & 257 & 293 \\
\hline 0 & -8 & -4 & -4 & 10 & 259 & 295 \\
\hline 0 & -8 & -4 & -4 & 11 & 252 & 288 \\
\hline 0 & -8 & -4 & -4 & 12 & 338 & 386 \\
\hline 0 & -8 & -4 & -4 & 12 & 280 & 319 \\
\hline 0 & -8 & -4 & -4 & 12 & 304 & 347 \\
\hline 0 & -8 & -4 & -4 & 12 & 279 & 318 \\
\hline 0 & -4 & -4 & 0 & 9 & 218 & 248 \\
\hline 0 & -4 & -4 & 0 & 9 & 254 & 290 \\
\hline 0 & -4 & -4 & 0 & 10 & 234 & 267 \\
\hline 0 & -4 & -4 & 0 & 11 & 265 & 303 \\
\hline 0 & -4 & -4 & 0 & 11 & 298 & 339 \\
\hline 0 & -4 & -4 & 0 & 11 & 255 & 291 \\
\hline 0 & -4 & -4 & 0 & 12 & 259 & 296 \\
\hline 0 & -4 & -4 & 0 & 12 & 279 & 319 \\
\hline 1 & -12 & -4 & -8 & 10 & 283 & 323 \\
\hline 1 & -12 & -4 & -8 & 10 & 261 & 297 \\
\hline 1 & -12 & -4 & -8 & 10 & 264 & 301 \\
\hline 1 & -12 & -4 & -8 & 12 & 312 & 356 \\
\hline
\end{tabular}




\begin{tabular}{|c|c|c|c|c|c|c|}
\hline $\boldsymbol{k}_{31}$ & $\begin{array}{c}\boldsymbol{\beta}_{1} \\
{[\text { grados] }}\end{array}$ & $\begin{array}{c}\boldsymbol{\beta}_{2} \\
{[\mathbf{g r a d o s}]}\end{array}$ & $\begin{array}{c}\boldsymbol{\delta}_{\boldsymbol{\beta}} \\
{[\mathbf{g r a d o s}]}\end{array}$ & $\begin{array}{c}\boldsymbol{U} \\
{[\mathbf{m} / \mathbf{s}]}\end{array}$ & $\begin{array}{c}\boldsymbol{\omega}_{3} \\
{[\mathbf{r a d} / \mathbf{s}]}\end{array}$ & $\mathbf{1 0}^{-3} \boldsymbol{R} \boldsymbol{c}$ \\
\hline 1 & -12 & -4 & -8 & 12 & 341 & 389 \\
\hline 1 & -12 & -4 & -8 & 12 & 312 & 356 \\
\hline 1 & -8 & -4 & -4 & 10 & 240 & 274 \\
\hline 1 & -8 & -4 & -4 & 10 & 275 & 314 \\
\hline 1 & -8 & -4 & -4 & 10 & 247 & 281 \\
\hline 1 & -8 & -4 & -4 & 12 & 286 & 326 \\
\hline 1 & -8 & -4 & -4 & 12 & 274 & 312 \\
\hline 1 & -8 & -4 & -4 & 12 & 324 & 370 \\
\hline 1 & -4 & -4 & 0 & 10 & 258 & 294 \\
\hline 1 & -4 & -4 & 0 & 10 & 135 & 154 \\
\hline 1 & -4 & -4 & 0 & 10 & 200 & 228 \\
\hline 1 & -4 & -4 & 0 & 12 & 224 & 255 \\
\hline 1 & -4 & -4 & 0 & 12 & 306 & 349 \\
\hline 1 & -4 & -4 & 0 & 12 & 160 & 183 \\
\hline
\end{tabular}




\section{BIBLIOGRAFÍA}

[1] Ball, A. J. Planetary Landers and Entry Probes. Cambridge University Press. 2007.

[2] Ball, A., Garry J., Lorenz, R., Kerzhanovich, V. Planetary Landers and Entry Probes. Cambridge University Press; 1 edition. 2007.

[3] Bartz, J., Miklosovic, D.S. An experimental analysis of camber efects of a 6-bladed flapped autorotational aerodynamic decelerator, AIAA 2003-2143. 17th AIAA Aerodynamic Decelerator Systems Technology Conference and Seminar, Monterey, California. 2003

[4] Butcher, J. C. Numerical methods for ordinary differential equations. John Wiley \& Sons. 2003.

[5] Chi-Tsong, C. Linear System Theory and Design, 3rd Edition, Oxford University Press. 1999.

[6] Crimi, P. Analysis of samara-wing decelerator steady-state characteristics, Journal of Aircraft, Vol. 25, No. 1, pp. 41-47. 1988.

[7] Cy Klein, A. H. A Small Student Designed Descent Probe. International Planetary Probe Workshop. 2006.

[8] Diebel, J. Representing Attitude: Euler Angles, Unit Quaternions, and Rotation Vectors. Stanford University. 2006.

[9] Dormand, J. R., Prince, P. J. A family of embedded Runge-Kutta formulae, Journal of Computational and Applied Mathematics. 1980.

[10] Dorrington G.E. Concept options for the aerial survey on Titan. ASR 47. 2011 
[11] Fang, A. C., Zimmerman, B. G. Digital Simulation of Rotational Kinematics. NASA TN D-5302. 1969.

[12] Greene, D. F. Johnson E. A. Seed mass and dispersal capacity in wind-dispersed diaspores, Oikos, vol. 67, no1. 1993.

[13] Hoerner, S. F., Borst, H.V. Fluid Dynamic Lift. Hoerner Fluid Dynamics, 1975.

[14] Horn, H. S., Nathan, R.A.N, Kaplan S. R. Long-distance dispersal of tree seeds by wind. Ecological research. Volume 16, Number 5, 877-885. 2001.

[15] Howard, A. Rotary Wing Decelerators. Tesis of Degree of Master of Science, College of Graduate Studies, University of Idaho. 2008.

[16] Jukubowski, A. K. A wind-tunnel study of spinning conical disk decelerators at Mach 4. Journal of Spacecraft, Vol. 11, N³. 1974.

[17] Karlen, L. Aerodynamics of a rotating body descending from the separation position of an artillery munition shell. Aerodynamic decelerator System Tecnology Conference. San Diego. 1991.

[18] Karlsen, L., Borgström, D., and Paulsson, L. Aerodynamics of a rotating body descending from the separation position of an artillery munition shell, 11 th AIAA Aerodynamic Decelerator Systems Technology Conference, AIAA-91-0870-CP, San Diego. 1991.

[19] Kellas, A. The Guided Samara: Design and Development of a Controllable SingleBladed Autorotating Vehicle. Tesis para el Master en Ciencia en Aeronáutica y Astronáutica en el Massachusetts Institute o Techlnology. 2007.

[20] Kuchemann, D. A Simple Method for Chordwise Loading on of any Given Aspect Calculating the Span and Straight and Swept Wings Ratio at Subsonic Speeds. D. Kuchemann. Reports and Memoranda No. 2935. 1952. 
[21] Kuchemann, D. The Aerodynamic Design of Aircraft. Pergamon Press. 1978.

[22] Lamar, J.E. Extension of Leading-Edge-Suction Analogy to Wings with Separated Flow Around the Side Edges at Subsonic Speeds. NASA TR R-428. 1974.

[23] Levin, D., Self-Induced Roll Oscillations of Low-Aspect-Ratio Rectangular Wings. Daniel Levin, Joseph Katzt. Journal of Aircraft. Vol. 29, No. 4. 1992.

[24] Levin, D., Shpund, Z. Dynamic Investigation of the Angular Motion of a Rotating Body-Parachute System, Journal of Aircraft, Vol. 32, №1. 1995.

[25] Lugt, H. J. Autorotation. Annual Review of Fluid Mechanics. Vol. 15: 123-147. 1983.

[26] Matlack, G.R. Diaspore Size, Shape, and Fall Behavior in Wind-Dispersed Plant Species. American Journal of Botany. Vol. 74, No. 8. 1987.

[27] McCutchen, C. W. The Spinning Rotation of Ash and Tulip Tree Samaras, Science, Vol. 197. 1977.

[28] McKinney, B. C. Launch vehicle with engine mounted on a rotor. Patent number: 5842665. 1998.

[29] Nadal Mora, V. Comportamiento aerodinámico de sondas atmosféricas en entornos aeroportuarios, Tesis Doctoral, Universidad Politécnica de Madrid, España, 2005.

[30] Nadal Mora V., Piechocki J., S Pezzotti. Ensayos en túnel aerodinámico de modelos de deceleradores aerodinámicos de alas rotatorias. CAIA 2, Córdoba, Argentina. 2010.

[31] Pelletier, A., Mueller, T. J. Low Reynolds Number Aerodynamics of Low-AspectRatio, Thin/Flat/Cambered-Plate Wings. Journal of Aircraft Vol. 37, Nro 5. 2000.

[32] Pepper, W. A new rotating parachute design having high performance, J. Spacecraft, Vol. 23, No. 2. 1986. 
[33] Piechocki, J., Nadal Mora V., Pezzotti S. Modelos teóricos y numéricos para predecir el comportamiento dinámico de pararrotores. CAIA 2, Córdoba, Argentina. 2010.

[34] Piechocki, J., Nadal Mora V., Pezzotti S. Estudio teórico del efecto del desplazamiento del plano que contiene a las palas con respecto al centro de masas en la dinámica del vuelo de un desacelerador aerodinámico de alas rotatorias. I CAIM, Bahía Blanca. 2008

[35] Richards, P.J., Nathan, W. Numerical Calculation of the 3-Dimensional Motion of Wind-borne Debris. Journal of Wind Engineering and Industrial Aerodynamics, Volume 96, Issue 10, Pages 2188-2202. 2007.

[36] Wick, B. H. Study of the Subsonic Forces and Moments on an Inclined Plate of Infinite Span. NACA TN 3221. 1951.

[37] Sanz Andres A., Nadal Mora V., Piechocki J. Pararrotors for planetary atmosphere exploration. IPPW-5, Burdeos, Francia. 2007.

[38] Steiner T. J., Young L. A. Rotary wing decelerator use on Titan. IPPW8, Portsmouth, EEUU. 2011.

[39] Thomson, W. T., Introduction to space dynamics. Dover Publications, 1986.

[40] Yasuda, K, Azuma, A. The Autorotation Boundary in the Flight of Samaras. Journal of Theoretical Biology, 185. 1997.

[41] Young L. A., Briggs G., Aiken E., Pisanich G. Rotary-Wing Decelerators for Probe Descent Through the Atmosphere of Venus. DTIC. 2004. 